\title{
INTERACTION MEASURES FOR SYSTEMS UNDER DECENTRALIZED CONTROL
}

Thesis by

Pierre Grosdidier

In Partial Fulfilment of the Requirements

for the Degree of

Doctor of Philosophy

California Institute of Technology

Pasadena, CA 91125

1986

(Submitted May 5, 1986) 
Mes études n' auraient pas été possibles sans le soutient infaillible de mes parents. A eux, je dédie cette thèse. 
iii

\section{ACKNOWLEDGMENTS}

I am grateful to my advisor, Professor M. Morari, for his advice, support and continuous encouragements throughout this work. I also wish to extend my thanks to S. Skogestad, J. Doyle and T. Skjellum for many useful and enthusiastic discussions. 


\begin{abstract}
Multivariable controllers are often avoided in the chemical process industries in favor of simpler diagonal or block-diagonal controllers. Such "decentralized" controllers are desirable because they result in control systems with fewer tuning parameters and greater failure tolerance. However, the ensuing simplicity in controller design must be weighted against the interactions which result from ignoring the off-diagonal system blocks. These can lead to performance deterioration and even instability. The purpose of an Interaction Measure (IM) is to indicate under what conditions the stability of the diagonal loops/blocks will guarantee that of the complete system.

One such measure, the Relative Gain Array (RGA), has found widespread acceptance both in industry and academia despite its empirical basis. This measure, in fact, has sound theoretical justifications. Rigorous relationships are derived in this study linking the RGA to closed-loop stability and robustness with respect to model uncertainty.

Using the notion of Structured Singular Value, a new dynamic IM is also defined for multi-variable systems under feedback with diagonal or block-diagonal controllers. This measure, the $\mu \mathrm{IM}$, can be used to select the "best" variable pairings for the controller as well as predict the stability of the decentralized control system. Its steady-state value also provides a sufficient condition for achieving offset-free performance with the closed-loop system. The relationship of this new IM with Rijnsdorp' IM and Rosenbrock's Direct Nyquist Array is clarified.
\end{abstract}

Finally, it is show how the $\mu \mathrm{IM}$, in conjunction with the RGA, can form the basis of a novel and useful methodology for the design of decentralized controllers. 


\section{TABLE OF CONTENTS}

ACKNOWLEDGMENTS

iii

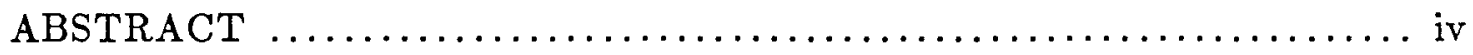

CHAPTER I: INTRODUCTION $\ldots \ldots \ldots \ldots \ldots \ldots \ldots \ldots \ldots \ldots \ldots \ldots \ldots \ldots \ldots \ldots \ldots \ldots \ldots$

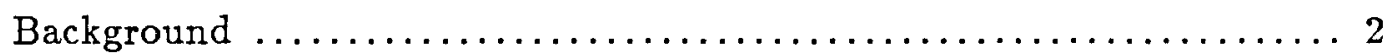

A New Approach to Decentralized Control ................... 4

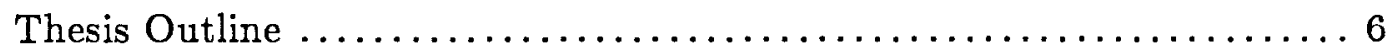

References $\ldots \ldots \ldots \ldots \ldots \ldots \ldots \ldots \ldots \ldots \ldots \ldots \ldots \ldots \ldots \ldots$

Figure Captions $\ldots \ldots \ldots \ldots \ldots \ldots \ldots \ldots \ldots \ldots \ldots \ldots \ldots \ldots \ldots$

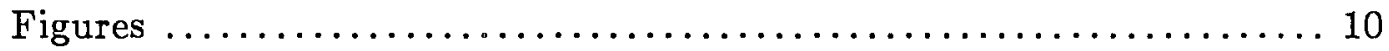

CHAPTER II: CLOSED-LOOP PROPERTIES FROM STEADY-STATE GAIN

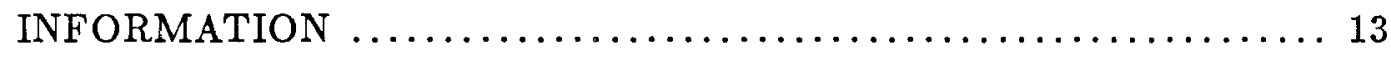

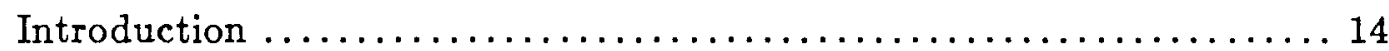

Definition and Algebraic Properties of the RGA $\ldots \ldots \ldots \ldots \ldots \ldots$

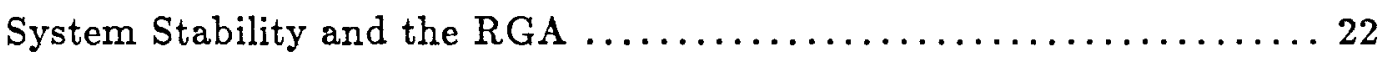

Integral Controllability and Failure Tolerance $\ldots \ldots \ldots \ldots \ldots \ldots \ldots . \ldots$

Right Half Plane Zeros and the RGA $\ldots \ldots \ldots \ldots \ldots \ldots \ldots \ldots$

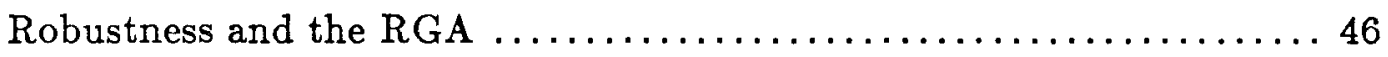

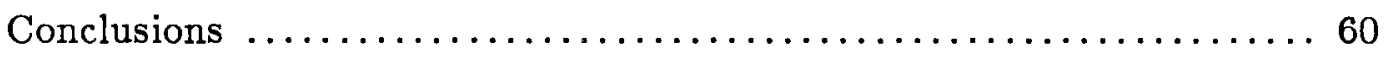

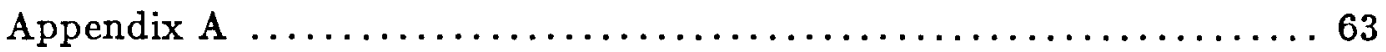

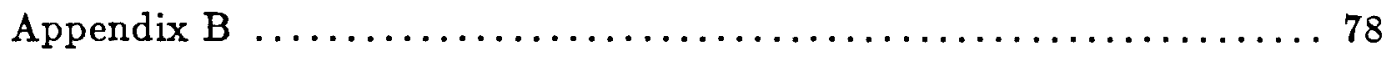

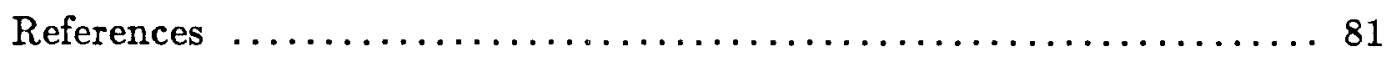

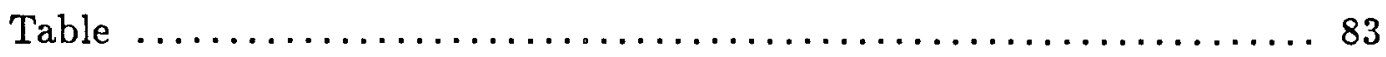

Figure Captions $\ldots \ldots \ldots \ldots \ldots \ldots \ldots \ldots \ldots \ldots \ldots \ldots \ldots \ldots \ldots \ldots$ 


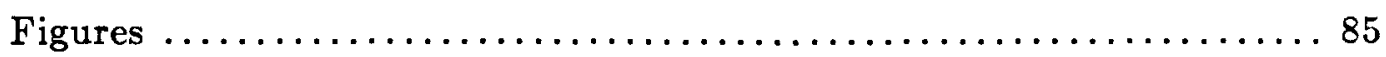

CHAPTER III: THE $\mu$ INTERACTION MEASURE $\ldots \ldots \ldots \ldots \ldots \ldots . . . \ldots 0$

Introduction $\ldots \ldots \ldots \ldots \ldots \ldots \ldots \ldots \ldots \ldots \ldots \ldots \ldots \ldots \ldots \ldots \ldots \ldots, \ldots 1$

Stability Conditions for Decentralized Control Systems ............ 95

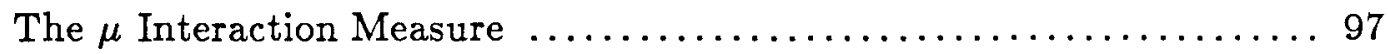

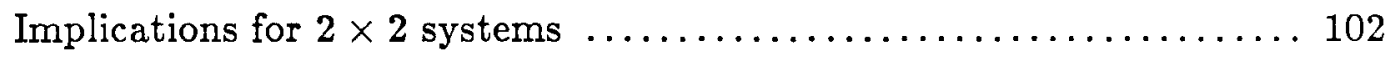

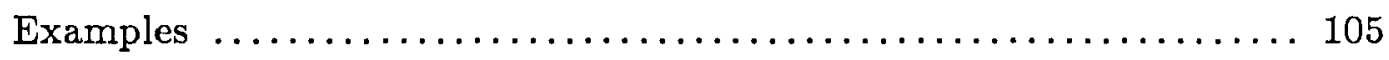

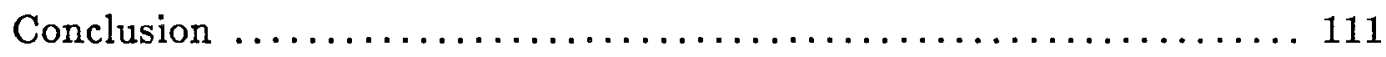

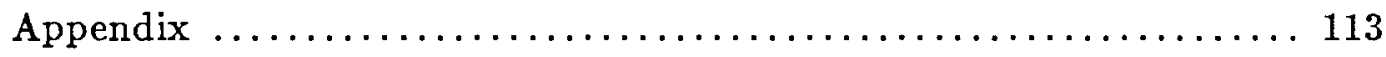

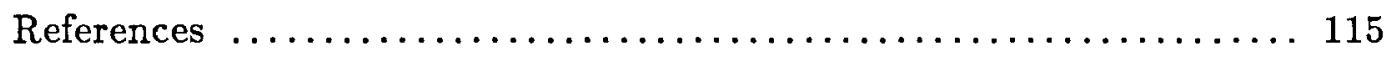

Tables $\ldots \ldots \ldots \ldots \ldots \ldots \ldots \ldots \ldots \ldots \ldots \ldots \ldots \ldots \ldots \ldots \ldots \ldots \ldots \ldots \ldots \ldots \ldots \ldots, 117$

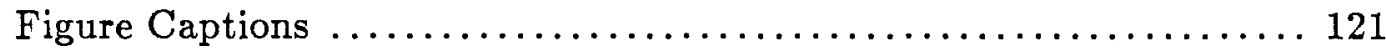

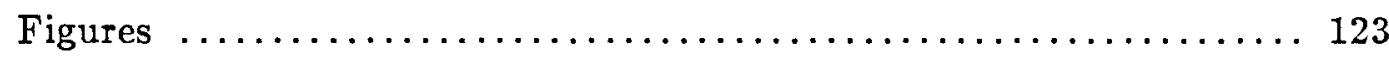

CHAPTER IV: INTERACTION MEASURES FOR SYSTEMS UNDER DECENTRALIZED CONTROL $\ldots \ldots \ldots \ldots \ldots \ldots \ldots \ldots \ldots \ldots \ldots \ldots \ldots \ldots \ldots$

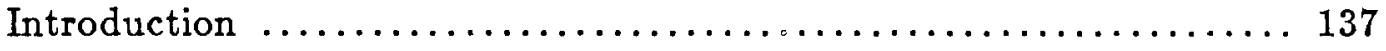

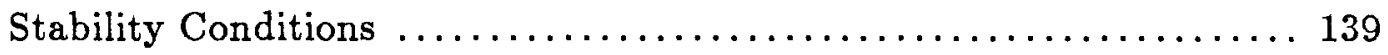

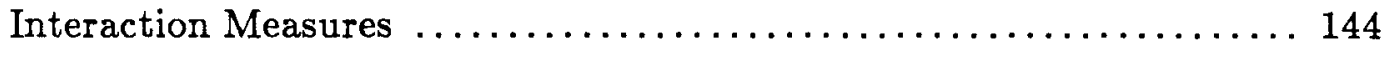

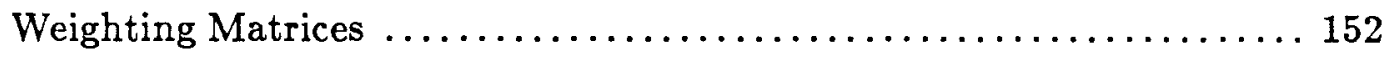

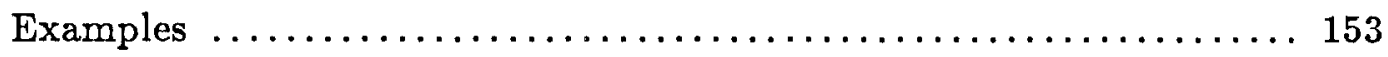

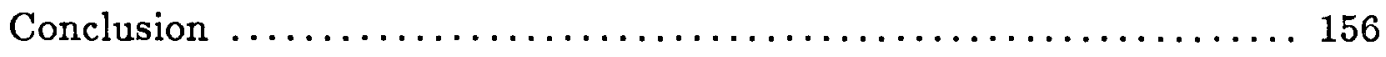

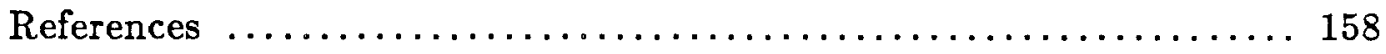

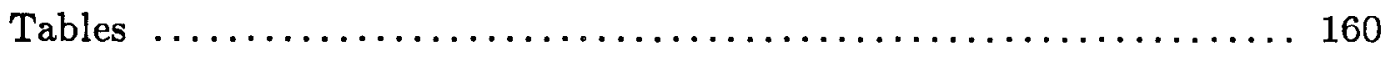

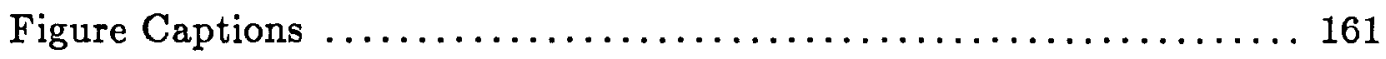

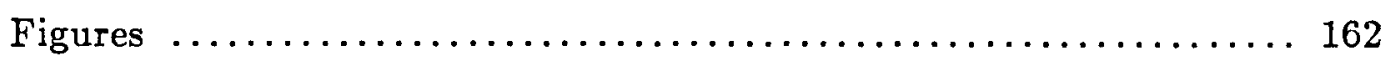


vii

CHAPTER V: A COMPUTER-AIDED METHODOLOGY FOR THE DESIGN OF DECENTRALIZED CONTROLLERS ................ 171

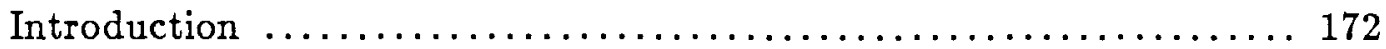

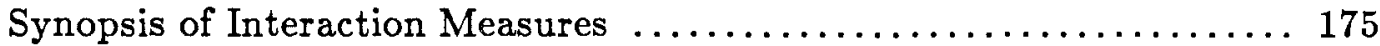

Decentralized Control System Design Methodology ............. 185

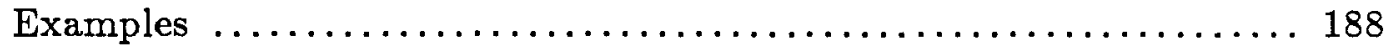

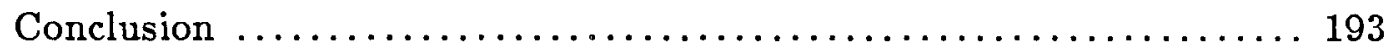

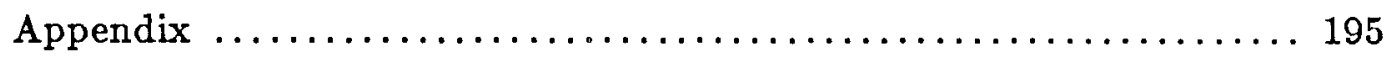

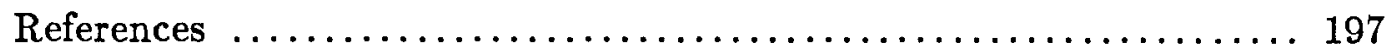

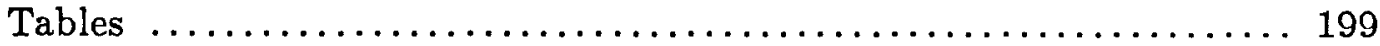

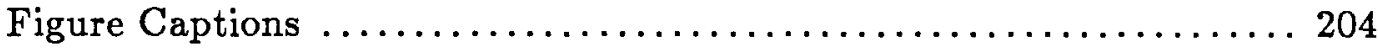

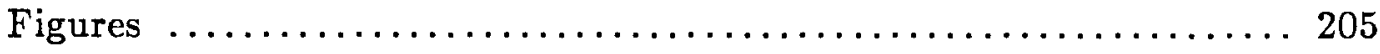

CHAPTER VI: CONCLUSIONS AND RECOMMENDATIONS . ...... 210 
CHAPTER I: INTRODUCTION 


\section{Background.}

The trend in the chemical process industries is toward the increasing use of automatic process control. This trend is mandated by the need to optimize plant operations for maximum profits. The availability of cheap and powerful microcomputers makes the widespread use of automatic control systems both technically possible and financially desirable. As a result, today's practicing control engineers are frequently faced with the task of designing controllers for plants with two or more input and output variables.

Ideally, a multivariable plant would be controlled by a single multivariable controller where the control action of each manipulated variable is a function of all the measurements (Fig. 1). In Fig. 1, $\mathbf{G}(s)=\left\{g_{i j}(s)\right\},(i, j=1, n)$ denotes the plant plant and $\mathbf{C}(s)=\left\{c_{i j}(s)\right\},(i, j=1, n)$ is the controller. The practice in the chemical process industries is to avoid multivariable controllers in favor of simpler controllers which have limited access to plant inputs and outputs. By rearranging these variables, it is always possible to present the controller in a diagonal form (Fig. 2). Such "decentralized" controllers are preferred because they result in control systems with fewer communication links and fewer tuning parameters. Furthermore, multivariable control theory has had little impact on process control until recently. As a result, the prevailing industrial philosophy is to control multi-input multi-output (MIMO) plants with a set of parallel singleinput single-output (SISO) proportional-integral-derivative (PID) controllers.

This intuitively appealing approach is not without its drawbacks. There are many outstanding examples where individually stable SISO loops yield unstable multivariable control systems when all loops are closed simultaneously. Importantly, for controllers with integral action, this phenomenon is independent of how loosely the controllers are tuned. The "interactions" between the SISO con-- 
trollers therefore create conditions that can destabilize otherwise stable systems. As a result of these interactions, the MIMO control system in Fig. 2 cannot, in general, be treated as a simple collection of SISO systems.

Attempts were made to alleviate this problem in order to retain the benefits of decentralized control. The pairing of controlled and manipulated variables soon was seen as an important factor in the degree of interactions. Interaction Measures (IM's) were suggested to quantify this effect. One such measure is the Relative Gain Array (RGA) which was introduced to assist pairing in a quantitative, although empirical manner (Bristol, 1966 ; Shinskey, 1967). The RGA is successful in providing pairing guidelines for systems with two inputs and two outputs. These guidelines make it possible to tune the two SISO controllers independently of one another. However, this is no longer true for systems larger than $2 \times 2$ and this limitation weights on the RGA's usefulness.

In the absence of a better alternative, current practice is to tune the SISO controllers $c_{i}(s),(i=1, n)$ in the diagonal multivariable controller by trial and error, and analyze the complete system

$$
\mathbf{H}(s)=\mathbf{G}(s) \mathbf{C}(s)(\mathbf{I}+\mathbf{G}(s) \mathbf{C}(s))^{-1}
$$

for stability and for performance. A recent publication by Alatiqi and Luyben (1985) examplifies this state of affairs.

Although this method of designing decentralized multivariable controllers suffices for many control problems, it fails to capture the real benefits of either decentralized or complex multivariable control. Tuning the diagonal controller to make only $\mathbf{H}(s)$ stable neglects the fact that single loop controllers are particularly suited for designing failure tolerant control systems. Moreover, the trial and error tuning effort may equal the work required to design a simple multivari- 
able controller, but has little hope of achieving the same quality of closed-loop performance.

\section{A New Approach to Decentralized Control.}

The design of a decentralized controller must require less trial and error and yield greater benefits than it does presently. It must be possible to tune each controller independently of the others and the resulting control system must be failure tolerant. These properties are desirable in the chemical industries because of recurring hardware failures (Roffel and Rijnsdorp, 1982) and because evolving plant operating conditions require frequent tuning adjustments.

The objective of this research effort is to show how a decentralized controller can be designed such that the closed-loop system has all these properties. This is done through a new, rigorous approach which emphasizes the use of diagonal and also block-diagonal controllers.

Our approach is motivated by the fact that by careful selection of the variable pairings, a multivariable control problem can, in effect, be reduced to several SISO or smaller MIMO problems. For example, consider, the control system shown in Fig. 3A for the $2 \times 2$ plant $\mathbf{G}(s)=\left\{g_{i j}(s)\right\},(i, j=1,2)$. It is physically reasonable to ignore the off-diagonal plant transfer functions $g_{12}(s)$ and $g_{21}(s)$ if these are small in magnitude relative to $g_{11}(s)$ and $g_{22}(s)$ (Fig. 2B). A decentralized controller that makes the closed-loop system in Fig. 2B stable should also stabilize the actual system in Fig. 2A with minimal loss of performance.

This example is important because it conveys the two key ideas that will allow us to develop a rigorous mathematical treatment of feedback systems with diagonal or block-diagonal controllers (Fig. 4). The first of these is that an $n \times n$ 
plant $\mathbf{G}(s)$ can be approximated by a diagonal or block-diagonal plant

$$
\tilde{\mathbf{G}}(s)=\operatorname{diag}\left(\mathbf{G}_{11}(s), \mathbf{G}_{22}(s), \ldots \mathbf{G}_{m m}(s)\right)
$$

if the off-diagonal elements or blocks of $\mathbf{G}(s)$ are sufficiently "small." Note that dominant elements or blocks can always be placed on the diagonal of $\mathbf{G}(s)$ by suitable row and column permutations. The second idea is that for sufficiently "close" plants $\mathbf{G}(s)$ and $\tilde{\mathbf{G}}(s)$, a diagonal or block-diagonal controller

$$
\mathbf{C}(s)=\operatorname{diag}\left(\mathbf{C}_{1}(s), \mathbf{C}_{2}(s), \ldots \mathbf{C}_{m}(s)\right)
$$

can be designed to make the feedback loop around $\tilde{\mathbf{G}}(s)$ stable (Fig. 5) with the assurance that the feedback loop around $\mathbf{G}(s)$ will also be stable (Fig. 4).

The selection of $\tilde{\mathbf{G}}(s)$ and the tuning of the controller blocks $\mathbf{C}_{i}(s),(i=1, m)$ are the major issues in the design of decentralized controllers. The basis of our approach is to rely on IM's to address each of these in a rigorous, quantitative manner. These IM's express the constraints imposed on the choice of $\tilde{\mathbf{G}}(s)$ and $\mathrm{C}(s)$ such that the stability of the block-diagonal system (Fig. 5)

$$
\tilde{\mathbf{H}}(s)=\tilde{\mathbf{G}}(s) \mathbf{C}(s)(\mathbf{I}+\tilde{\mathbf{G}}(s) \mathbf{C}(s))^{-1}
$$

guarantees that of the complete system (Fig. 4)

$$
\mathbf{H}(s)=\mathbf{G}(s) \mathbf{C}(s)(\mathbf{I}+\mathbf{G}(s) \mathbf{C}(s))^{-1}
$$

The benefits of this approach are that it yields decentralized control systems with the following desirable properties:

(1) Decentralized tuning. The controllers $\mathbf{C}_{i}(s),(i=1, m)$ are tuned to make each of the closed-loop systems $\mathbf{H}_{i}(s)=\mathbf{G}_{i i}(s) \mathbf{C}_{i}(s)\left(\mathbf{I}+\mathbf{G}_{i i}(s) \mathbf{C}_{i}(s)\right)^{-1}$ stable. 
(2) Failure tolerance. The control system remains stable when failure occurs in any number of feedback loops. In this event, it is assumed that the failures are recognized and the corresponding controller blocks are placed in manual. Unfortunately, IM's are only analysis tools. They can only indicate whether a given $\tilde{\mathbf{G}}(s)$ and $\mathbf{C}(s)$ yield a control system with the desired stability and decentralized tuning properties. The designer's task is to search for a suitable $\tilde{\mathbf{G}}(s)$ and $\mathbf{C}(s)$ within the guidelines set by the IM's. Therefore our approach does not completely remove the element of trial and error presently required for designing decentralized controllers. In addition, IM's provide tuning guidelines that guarantee the stability of $\mathbf{H}(s)$ but not its performance. Therefore, closed-loop responses to disturbance and set-point changes can be very badly behaved. This point will be emphasized in the thesis and possible remedies will be presented. Synthesis methods for selecting $\tilde{\mathbf{G}}(s)$ and $\mathbf{C}(s)$ are obviously desirable but are not addressed here and remain an outstanding issue for future work.

\section{Thesis Outline.}

This introduction motivates the use of decentralized controllers and suggests the need for a new approach to their design. This approach is presented in the thesis in the following manner. Chapter II gives a thorough and rigorous mathematical treatment of the RGA, the oldest and most popular of existing IM's. Emphasis is placed on its relationship to closed loop stability and on a new result showing its usefulness as a measure of closed-loop robustness with respect to plant model uncertainty. The latter does not pertain strictly to decentralized control and is included for the sake of thoroughness and to demonstrate the RGA's versatility. We subsequently present the first (corrected) statement and proof of Niederlinski's theorem. Chapter II also develops the concept of Integral 
Controllability following Morari (1985), although its usefulness to decentralized control is only discussed in Chapter V. In Chapter III we motivate the use of IM's and formulate our approach for solving the decentralized control problem. This approach leads us to derive necessary and sufficient conditions for the stability of both $\mathbf{H}(s)$ and $\tilde{\mathbf{H}}(s)$, and to subsequently define the $\mu$ IM. This new IM exploits in a unique way the notion of Structured Singular Values introduced by Doyle (1982). We demonstrate how this IM can be used both for selecting $\tilde{\mathbf{G}}(s)$ and for tuning the controllers $\mathbf{C}_{i}(s),(i=1, m)$. Finally in this chapter, we prove that Shinskey's tuning rule (Shinskey, 1979) using the RGA for a certain class of $2 \times 2$ systems is theoretically justified. Note that all theorem proofs in this chapter will be found in Chapter IV. We formulate a unified treatment of other existing IM's in Chapter IV and derive the relationship between the $\mu$ IM and Rosenbrock's Direct Nyquist Array (1974). We also derive in this chapter a second proof of Niederlinski's theorem. This proof is a generalization of Niederlinski's original result to block-diagonal control systems and open loop unstable plants. Chapter $\mathrm{V}$ presents a synopsis of existing IM's. We show how the most useful of these (the RGA and the $\mu$ IM) can be used as the basis of a novel and useful methodology for the design of decentralized controller. In Chapter VI we summarize the benefits and drawbacks of our approach to the decentralized control problem and suggest areas for future research. 


\section{REFERENCES}

Alatiqi, I. M. and W. L. Luyben (1985). Control of a Complex Sidestream Column/Stripper Distillation Configuration. Accepted for publication, Ind. Eng. Chem. Process Des. Dev.

Bristol, E. H. (1966). On a New Measure of Interaction for Multivariable Control. IEEE Trans. Autom. Cont., AC-11, 133-134.

Doyle, J. C. (1982). Analysis of Feedback Systems with Structured Uncertainties. IEE Proc., 129-D, 242-250.

Morari, M. (1985). Robust Stability of Systems with Integral Control. IEEE Trans. Autom. Cont., AC-30, 574-577.

Roffel, B. and J. E. Rijnsdorp (1982). Process Dynamics, Control and Protection. Ann Arbor Science Publishers, Ann Arbor.

Rosenbrock, H. H. (1974). Computer-Aided Control System Design. Academic Press, London.

Shinskey, F. G. (1967). Process Control Systems. McGraw-Hill Book Co., New York.

Shinskey, F. G. (1979). Process Control Systems. 2nd Ed. McGraw-Hill Book Co., New York. 


\section{FIGURE CAPTIONS}

Figure 1. Feedback system with general multivariable controller.

Figure 2. Feedback system with diagonal multivariable controller.

Figure 3A. Decentralized control structure for a $2 \times 2$ system with interactions.

Figure $3 B$. Decentralized control structure for a $2 \times 2$ system without interactions.

Figure 4. General decentralized control structure with interactions.

Figure 5. General decentralized control structure without interactions. 


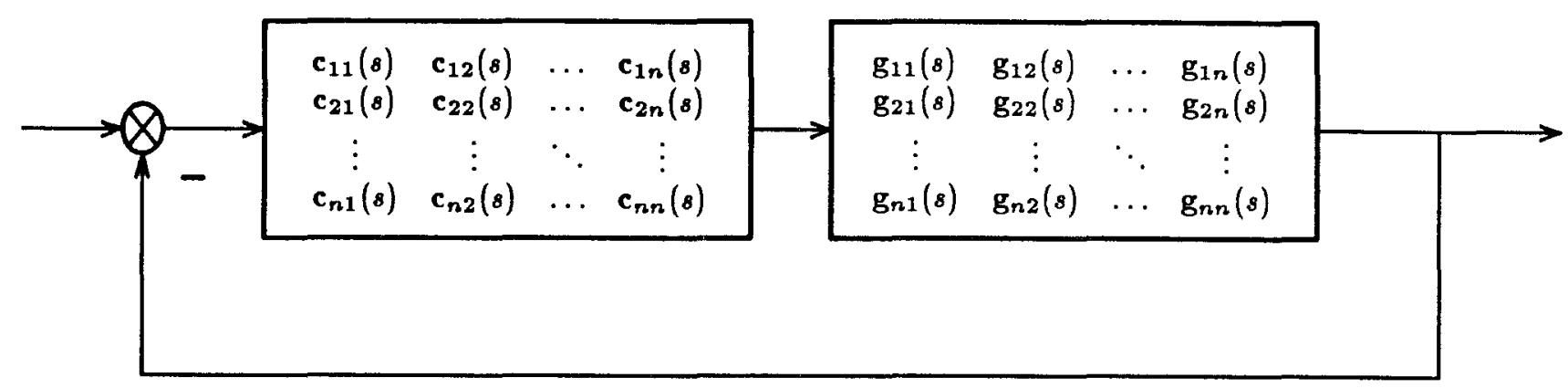

Figure 1. Feedback system with general multivariable controller.

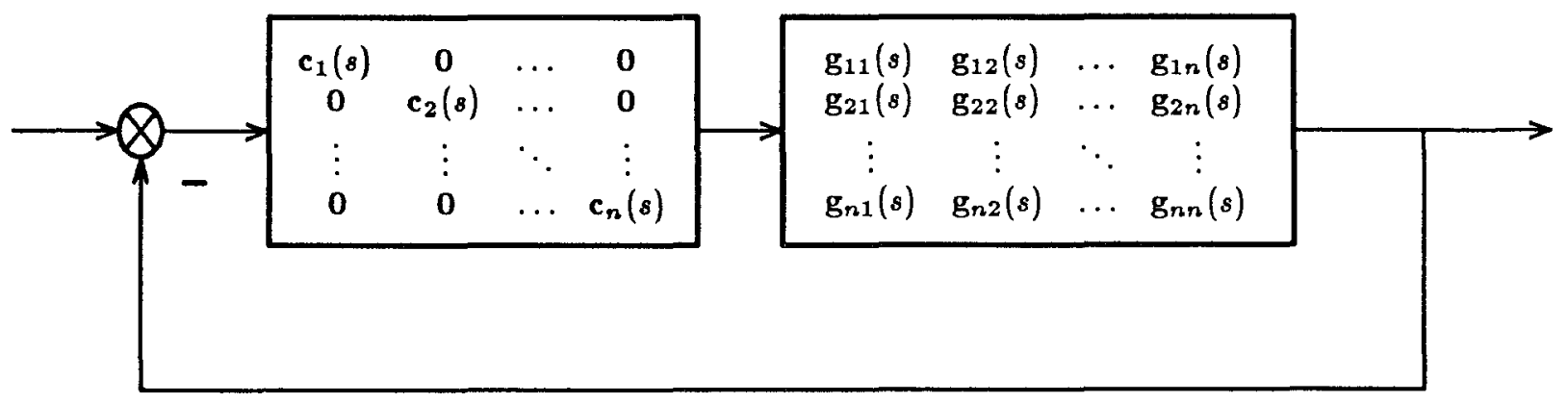

Figure 2. Feedback system with diagonal multivariable controller. 


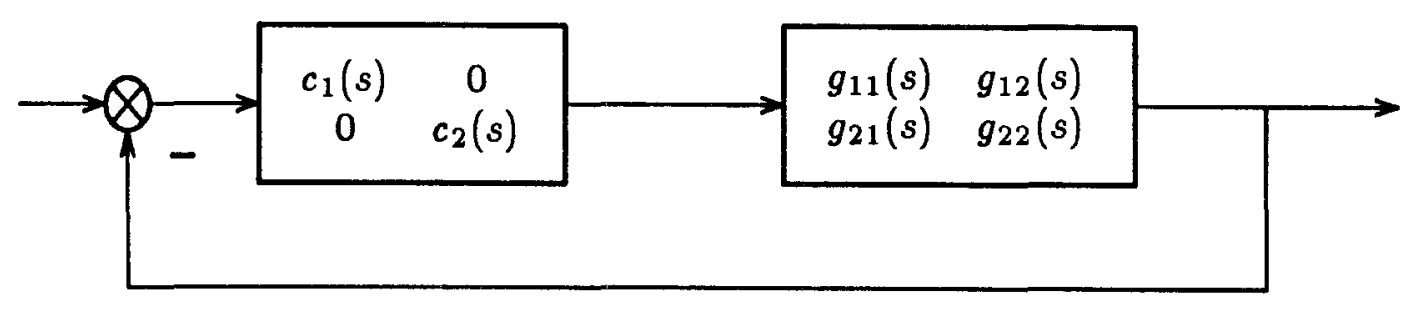

Figure 3A. Decentralized control structure for a $2 \times 2$ system with interactions.

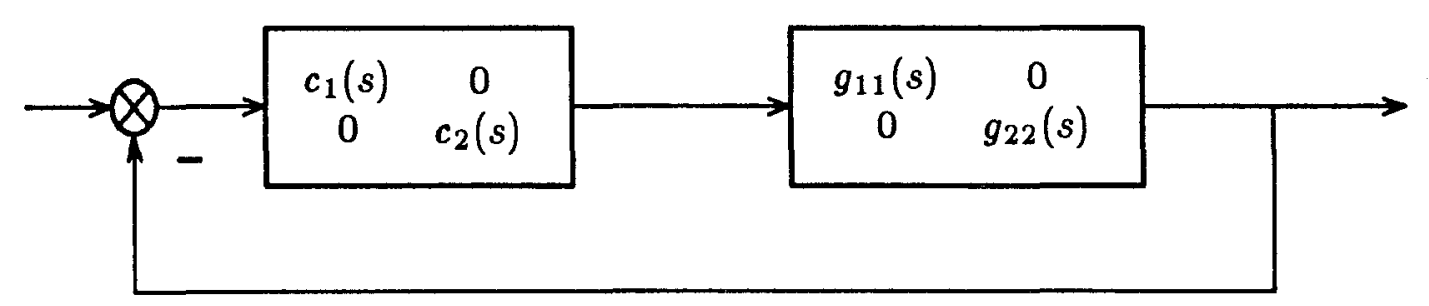

Figure 3B. Decentralized control structure for a $2 \times 2$ system without interactions. 


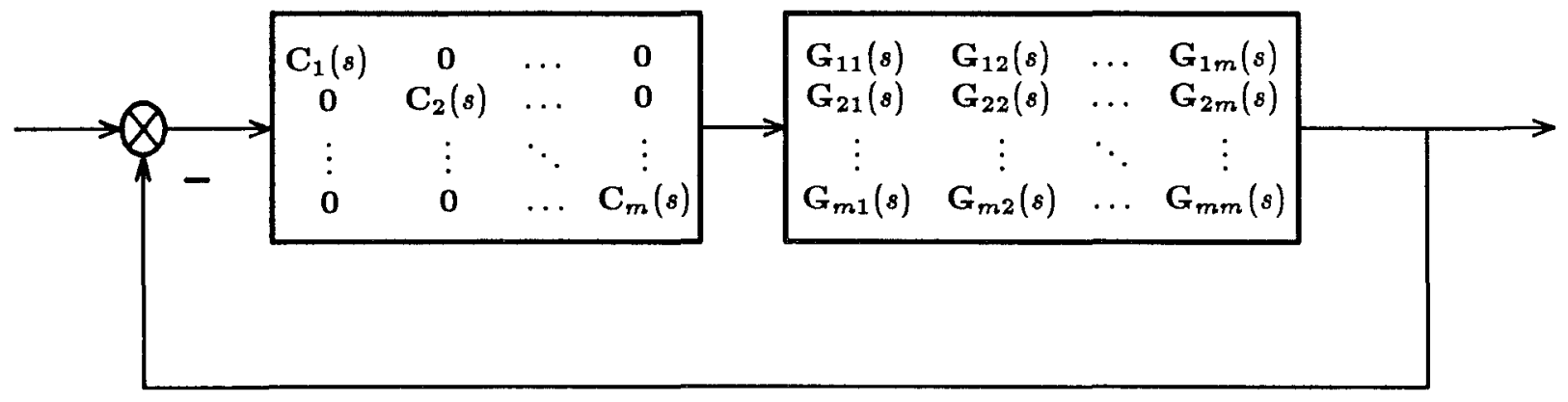

Figure 4. General decentralized control structure with interactions.

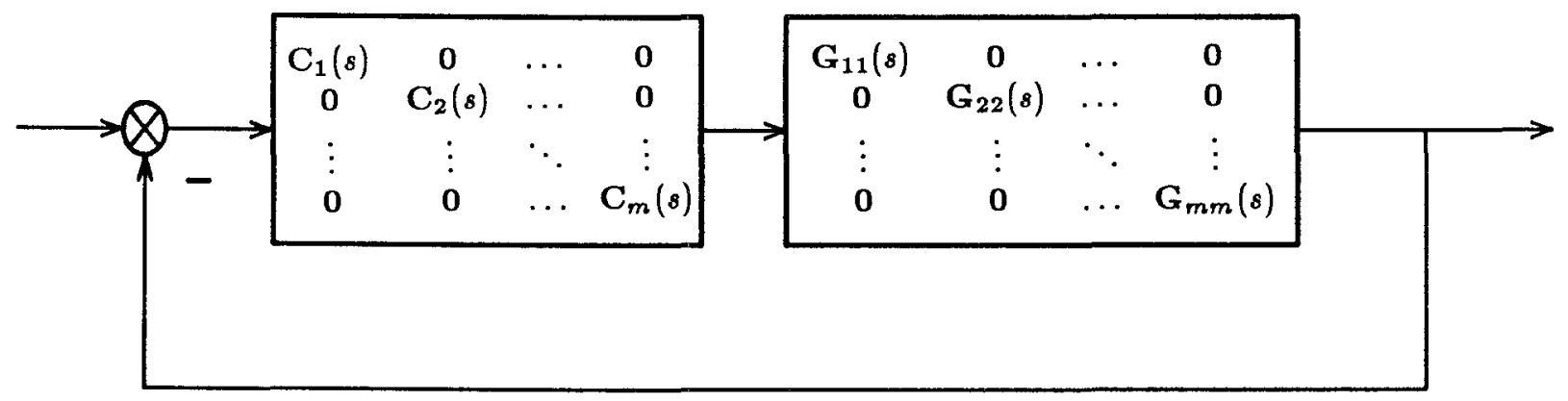

Figure 5. General decentralized control structure without interactions. 
CHAPTER II: CLOSED-LOOP PROPERTIES FROM STEADY-STATE GAIN INFORMATION 
INTRODUCTION

Modeling uncertainties and constantly changing operating conditions make it very difficult to develop reliable dynamic models for chemical processes. Often, only steady-state gain information is available. In multi-input multi-output (MIMO) systems, these data may be represented as a matrix of steady-state gains $\underline{\underline{G}}(0)$. Since this matrix $\underline{\underline{G}}(0)$ is often the only information available on the system, any method that will allow the extraction of useful feedback properties from it is clearly of great practical importance. The steady state gain matrix can, for example, be used to evaluate a measure of steady state interactions between controlled and manipulated variables. This measure of interactions, the Relative Gain Array (RGA), has found widespread acceptance both in industry and in academia since its introduction almost twenty years ago (Bristo1, 1966 and Shinskey, 1967). This popularity is chiefly a result of the array's simplicity and empirically confirmed reliability. However, in spite of wide acceptance and abundant studies on practical applications (McAvoy, 1983), the RGA remains an empirical tool with little or no rigorous theoretical basis

In this article, novel analysis techniques are presented to show that very important closed loop properties can easily be extracted from the steady-state gain matrix. These techniques have been developed for open loop stable systems subject to feedback by controllers which include integral action. The properties comprise closed-loop stability, sensor and actuator failure tolerance, feasibility of decentralized control structures and robustness with respect to modeling errors. It will be shown that some of the results can be rigorously expressed in terms of the RGA. This will show that the RGA has, in fact, sound theoretical justifications and is much more than a simple measure of interactions. 
The approach in this paper is as follows. We shall first provide a definition of the RGA and state some of its properties. It will subsequently be shown how the RGA can be used to predict the closed loop instability of multivariable control systems. Next, a new analysis technique will be presented which is able to extract much more stability information from the steady-state gain matrix than the RGA. Next, the relationship between Right Half Plane (RHP) zeros and the RGA will be briefly examined. Finally, a new theorem will show how the RGA can be used to predict the sensitivity of a multivariable system to modeling errors.

Many of the results presented in this paper were conjectured previously by other researchers. However, as we shall show, some of these conjectures are incorrect, some are partially correct, and some are correct but the arguments used to prove them are incorrect. To avoid any misunderstanding based on past misconceptions all the definitions and properties of the RGA known to date wi11 be restated.

Throughout this article, it is assumed that we are dealing with square (nxn), open loop stable and non-singular transfer matrices. These will be denoted with the bold-face letter $\underline{G}(s)$ while their individual elements will be denoted by $g_{i j}(s)$. Moreover, $\underline{G}^{i j}(s)$ will denote the matrix $\underline{\underline{G}}(s)$ with its ith row and $j$ th column removed. Steady state values of these variables will be denoted by the same characters without the "s", i.e. $\underline{\underline{G}}(0)=\underline{\underline{G}}$ and $g_{i j}(0)=g_{i j}$. Inputs and outputs (manipulated and controlled variables) are denoted by $u_{i}$ and $y_{i}$, respectively, when dealing with scalars and by $\underline{u}$ and $y$ when dealing with vectors of variables. In all cases, it is assumed that these variables represent deviations from the steady state. Finally, the open right half complex plane will be denoted by $\underline{C}^{+}$and the open left half complex plane by $\mathrm{C}^{-}$. 
DEFINITION AND ALGEBRAIC PROPERTIES OF THE RGA Definition of the RGA as an Interaction Measure

In this section, to demonstrate the basic assumptions underlying the RGA, its derivation will be carried out for $2 \times 2$ systems in extensive detail. Consider the transfer matrix $\underline{G}(s)$ with elements $g_{i j}(s)$, inputs $u_{i}$ and outputs $y_{j} ;(i, j=1,2)$. In the absence of any controller on the system, the transfer function between input $u_{1}$ and output $y_{1}$ is simply (Figure 1):

$$
\left(\mathrm{y}_{1} / \mathrm{u}_{1}\right)_{\mathrm{OL}}=\mathrm{g}_{1.1}(\mathrm{~s})
$$

where OL indicates "open loop".

Now, suppose that it is desired to control output $y_{2}$ with input $u_{2}$ by installing a controller with the transfer function $\mathrm{gc}_{2}(\mathrm{~s})$ (Figure 2 ). In the presence of this controller, the transfer function between $u_{1}$ and $y_{1}$ changes. It becomes the sum of the open-loop transfer function $g_{11}(s)$ and the transfer function created by the control loop (dotted line on Figure 2):

$$
\left(y_{1} / u_{1}\right)_{\text {CL22 }}=g_{11}(s)-\frac{g_{c_{2}}(s) g_{12}(s) g_{21}(s)}{1+g c_{2}(s) g_{22}(s)}
$$

The subscript CL22 in Equation (2) indicates that the loop between $\mathrm{u}_{2}$ and $\mathrm{y}_{2}$ is closed.

The extent to which the transfer function between $\mathrm{u}_{1}$ and $\mathrm{y}_{1}$ is affected by the presence of the control loop can be measured by the ratio of these last two expressions. Calling this ratio $\mu_{11}(\mathrm{~s})$ : 


$$
\mu_{11}(s)=\frac{\left(y_{1} / u_{1}\right) \text { oL }}{\left(y_{1} / u_{1}\right) \text { CL } 22}=\frac{g_{11}(s)+g_{2}(s) g_{11}(s) g_{22}(s)}{g_{11}(s)+g c_{2}(s)\left[g_{11}(s) g_{22}(s)-g_{12}(s) g_{21}(s)\right]}
$$

Rearranging ( $3 a)$

$$
\left(\frac{\mathrm{y}_{1}}{\mathrm{u}_{1}}\right)_{\mathrm{CL}}=\frac{1}{\mu_{11}(\mathrm{~s})}\left(\frac{\mathrm{y}_{1}}{\mathrm{u}_{1}}\right)_{\mathrm{OL}}=\frac{1}{\mu_{11}(\mathrm{~s})} \mathrm{g}_{11}(\mathrm{~s})
$$

$\mu_{11}^{-1}$ can be interpreted as a multiplicative perturbation of the transfer function $\left(\frac{\mathrm{y}_{1}}{\mathrm{u}_{1}}\right)$ oL caused by the closed control 1oop. This relationship is represented schematically in Fig. 3. $\mu_{11}(\mathrm{~s})$ is difficult to use as a measure of interaction; Equation (3a) shows that it depends not only on the transfer matrix elements but also on the controller $\mathrm{gc}_{2}(\mathrm{~s})$ and furthermore, is a function of frequency. Thus it is convenient to make assumptions both on the controller and on the frequency in order to define a simpler measure.

In view of typical process control requirements it is reasonable to assume that the controller includes integral action, i.e., that the controller transfer function contains the factor $s^{-1}$. To minimize the modeling requirements the interaction measure $\mu_{11}(s)$ is evaluated at zero frequency $(s=0)$. Under these assumptions, the controller $\mathrm{gc}_{2}(\mathrm{~s})$ has infinite steady state gain $\left(\mathrm{gc}_{2}(0)=\infty\right)$ and the measure of interaction $\mu_{11}(\mathrm{~s})$ (now denoted by $\lambda_{11}$ ) can be expressed solely in terms of the elements of the steady state gain matrix $\underline{\underline{G}}$.

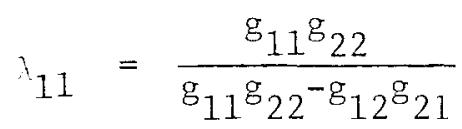

of course, similar analyses can be performed for other permutations of the variables and a relative gain $\lambda_{i j}$ can be obtained for input $u_{j}$ and output $y_{i} ;(i, j=1,2)$. For a square transfer matrix $\underline{G}$, a matrix of relative gains $\Lambda$, of the same size can be evaluated. This matrix is known as the Relative Gain Array for the transfer matrix $\mathrm{G}$. 
From the definition of the relative gain, it is clear that the system interactions, the perturbations caused by the closed control loops, will be minimized for those pairs of variables with relative gains of unity. As the magnitude of the relative gain $\lambda_{i j}$ between two variables $u_{j}$ and $y_{i}$ departs from unity, more interactions must be expected. For this reason, variables should be paired when their relative gain is as close to unity as possible.

When the relative gain between two variables is greater than zero, interactions between the two variables are said to be positive. Alternatively, negative relative gains imply negative interactions (Ray, 1981). In this case, the steady state effect of an input $u_{j}$ on an output $y_{i}$ in the closed loop case is opposite to that in the open loop case.

We chose this method of deriving the RGA over others to emphasize that the use of controllers with integral action is inherent in the definition of the RGA. This fact is sometimes referred to as the "perfect control requirement". A better term would be "perfect steady state control requirement" because the integral contro1 action leads to offset-free, that is "perfect steady state" control.

Extension of the RGA to Larger Systems

The concluding discussion in the last section leads to an alternate definition of the RGA which is particularly suitable for its extension to larger systems.

Consider an nxn transfer matrix $\underline{\underline{G}}(s)$ with inputs $u_{i}$ and outputs $y_{i}$; $(i=1, n)$, and consider specifically one input $u_{j}$ and one output $y_{i}$. 
Definition 1: The relative gain between an input $u_{j}$ and an output $y_{i}$ is the ratio of two steady state gains: The steady state gain between $u_{j}$ and $y_{i}$ when no control is applied to the system and the steady state gain between the same variables $u_{j}$ and $y_{i}$ when feedback control involving all other inputs $u_{k}(k=1, n ; k \neq j)$ and all other outputs $y_{\ell}(\ell=1, n ; \ell \neq i)$ is applied to the system such that in the steady state all $\mathrm{y}_{\ell}(\ell=1, \mathrm{n} ; \ell \neq i)$ are held at their nominal value (no off-set).

This definition can be expressed mathematically as

$$
\lambda_{i j}=\frac{\left(\partial y_{i} / \partial u_{j}\right) u_{k}=0, k \neq j}{\left(\partial y_{i} / \partial u_{j}\right)_{y_{l}}=0, \ell \neq i}
$$

and assumes that the matrix $\underline{\underline{G}}^{i j}$ is non-singular.

For an nxn transfer matrix $\underline{G}$, nxn relative gains are obtained to form the Relative Gain Array $\stackrel{\Lambda}{=}$.

By rewriting Equation (5) as:

$$
\lambda_{i j}=\left.\left.\frac{\partial y_{i}}{\partial u_{j}}\right|_{u_{k}=0, k \neq j} \cdot \frac{\partial u_{j}}{\partial y_{i}}\right|_{y_{\ell}=0, \ell \neq i}
$$

and using the identities:

$$
\begin{aligned}
& \left.\frac{\partial y_{i}}{\partial u_{j}}\right|_{u_{k}=0, k \neq j}=g_{i j} \\
& \left.\frac{\partial u_{j}}{\partial y_{i}}\right|_{y_{l}=0, \ell \neq i}=\hat{g}_{j i}
\end{aligned}
$$

the following expression for $\lambda_{i j}$ is obtained:

$$
\lambda_{i j}=g_{i j} \cdot \hat{g}_{j i}
$$


In these last two expressions, the term $\hat{g}_{j i}$ denotes the $(j, i)$ th element of the matrix $\underline{\underline{G}}^{-1}$.

Thus from the knowledge of the steady state transfer matrix $\underline{\underline{G}}$ and its inverse, the Relative Gain Array $\Lambda$ can be promptly evaluated.

\section{Algebraic Properties of the Relative Gain Array}

Property 1: The sum of the elements of each row and each column of the relative gain array is always unity.

Proof: From $\underline{G}^{-1} \cdot \underline{\underline{G}}=\underline{\underline{I}}, \sum_{j=1}^{n} \hat{\mathrm{g}}_{i j} \cdot g_{j k}=\delta_{i k}$

$$
\begin{aligned}
& \text { Thus } \sum_{j=1}^{n} \hat{g}_{i j} \cdot g_{j i}=\sum_{j=1}^{n} \lambda_{i j}=1 \\
& \text { Similarly from } \underline{\underline{G}} \cdot \underline{\underline{G}}^{-1}=\underline{\underline{I}} ; \sum_{i=1}^{n} \lambda_{i j}=1
\end{aligned}
$$

Property 2: Any permutation of rows and columns in a transfer matrix $\underline{G}$ results in the same permutation in the $\mathrm{RGA}$. Mathematically, if $\underline{=}=\mathrm{RGA}(\underline{G})$, if $\stackrel{\mathrm{P}}{=}_{1}$ and $\stackrel{\mathrm{P}}{=}_{2}$ are two permutation matrices, and if $\underline{\Lambda}^{\prime}=\operatorname{RGA}\left(\underline{\mathrm{P}}_{1} \stackrel{\mathrm{G}}{=} \underline{\mathrm{P}}_{2}\right)$, then

$$
\stackrel{\Lambda}{=}^{\prime}=\underline{\underline{P}}_{1} \stackrel{\Lambda}{=} \underline{\underline{P}}_{2}
$$

Proof: The coefficients $\lambda_{i j}$ of the RGA can be expressed as

$$
\lambda_{i j}=\frac{g_{i j} * c_{i j}}{\operatorname{det} \underline{G}}
$$

where $c_{i j}$ denotes the $(i, j)$ th cofactor of $\underline{G}$. Since any row or column permutation in $\underline{\underline{G}}$ results in the same permutation in the matrix of cofactors, it is clear from Equation (10) that the permutation carries over to the RGA. 
Property 3: The RGA is invariant under input and output scaling. By scaling we mean pre- and post-multiplication of $\underline{G}$ by diagonal matrices $\underline{S}_{1}$ and $\underline{\underline{S}}_{2}$. Mathematically, if $\underline{\Lambda}=\operatorname{RGA}(\underline{\underline{G}})$ and if $\underline{\Lambda}^{\prime}=\operatorname{RGA}\left(\underline{\mathrm{S}}_{1} \stackrel{\mathrm{G}}{\underline{\underline{S}}} \underline{\underline{S}}_{2}\right)$, then $\bigwedge^{\prime}=\Lambda$

Proof: $\quad \lambda_{i j}^{\prime}=g_{i j}^{\prime} \hat{g}_{j i}^{\prime}$

$$
\begin{aligned}
& =s_{1 i} s_{2 j} g_{i j} \cdot \frac{1}{s_{2 j}} \cdot \frac{1}{s_{1 i}} \cdot \hat{g}_{j i} \\
& =\lambda_{i j}
\end{aligned}
$$

Property 4: If the transfer matrix is diagonal or triangular, then $\underline{\underline{\Lambda}}=\underline{\underline{I}}$. In the former case, the system is said to be decoupled while in the latter case it is known as one way interactive.

Property 5: For a 2x2 transfer matrix with no zero elements, two special cases arise:

(a) If there is an odd number of positive elements in $\underline{\underline{G}}$, then $\lambda_{i j} \varepsilon(0,1) ; i, j=1,2$.

(b) If there is an even number of positive elements in $\underline{G}$, then $\lambda_{i j} \varepsilon(-\infty, 0) \cup(1, \infty) ; i, j=1,2$. 
SYSTEM STABILITY AND THE RGA

Interactions in a multivariable control system have implications beyond merely propagation of disturbances between loops. Consider for example a 2 input/output system where the controllers have been tuned independently. Even though the individual performance of the two controllers when tuned may be quite satisfactory, the overall system will sometimes go unstable when both loops are operated together. The interacting controllers therefore create conditions which can destabilize an otherwise stable system.

Such behavior is now qualitatively well understood (Shinskey, 1977 and Shinskey, 1979). Moreover, Shinskey has shown through semi-quantitative arguments that it can be interpreted and predicted through the RGA. Thus it appears that the RGA can be used not just as a measure of variable interactions but also as a measure of system stability.

Unfortunately, in spite of Shinskey's encouraging results, there have been very few attempts to formulate rigorous relationships linking the RGA to the stability of multivariable control systems. Clearly such relationships would be of great benefit considering the ease of calculation of the RGA and the importance of the stability information. The objective of this section will be to investigate the existence of such relationships.

Since in the derivation of the RGA it was assumed that the output variables were subject to perfect steady-state control, it seems natural to seek a relationship between the RGA and systems under integral control. We shall begin this section by establishing the conditions under which these systems can be made stable. This will lead to the concepts of integral stabilizability and failure sensitivity. The RGA will subsequently be examined in 1ight of these concepts. 
Integral Controllers and Integral Stabilizability

In the present context, the term integral controller is used to designate PI and PID controllers or any multivariable feedback controller which includes integral action. Al1 such controllers can be decomposed into a matrix of integrators $\mathrm{k} / \mathrm{s} \cdot \underline{\underline{I}}$ and a compensator matrix $\underline{\underline{C}}(\mathrm{~s})$. Here, $\mathrm{k}$ is a positive constant and $\underline{\underline{I}}$ is the identity matrix. In the case of a single variable PI controller, for example, $\underline{\underline{I}}=1$ and $\underline{\underline{C}}(s)=\left(\tau_{i} s+1\right) / \tau_{i}$, where $\tau_{i}$ is the reset time. Such decomposition simplifies the analysis since we are not interested in a specific compensator but rather in the general consequences of the integral action. Next, we consider the nxn plant transfer matrix $\underline{G}(s)$ and the control configuration shown in Figure 4. Define $\underline{H}(s)=\underline{\underline{G}}(s) \underline{\underline{C}}(s)$. At this point, it must be mentioned that if $\underline{\underline{G}}(s)$ contains semiproper ${ }^{*}$ ) elements, the corresponding $\underline{\underline{H}}(\mathrm{~s})$ may be improper $\left.{ }^{*}\right)$. This is a consequence of the definition of $\stackrel{\mathrm{C}}{\underline{(s}}$ ).

Definition 2: The system $\stackrel{\mathrm{H}}{=}(\mathrm{s})=\underline{\mathrm{G}}(\mathrm{s}) \underline{\mathrm{C}}(\mathrm{s})$ is called integral stabilizable if there exists a $k>0$ such that the closed loop system shown in Figure 4 is stable and has zero tracking error for all asymptotically constant inputs. Theorem 1 states a necessary condition for integral stabilizability.

Theorem 1: Assume $\underline{\underline{H}}(\mathrm{~s})$ is a $\operatorname{proper}^{*}$ ) rational transfer matrix. $\underline{\underline{H}}(\mathrm{~s})$ is integral stabilizable only if $\operatorname{det}(\underline{\underline{H}}(0))>0$.

Proof: See Appendix A

*) If $\lim _{S \rightarrow \infty} \underline{G}(s)=\underline{\underline{0}}, \underline{\underline{G}}(\mathrm{~s})$ is said to be strictly proper. If $\lim _{\mathrm{S} \rightarrow \infty} \underline{\underline{G}}(\mathrm{~s})=\underline{M}$, where $\stackrel{M}{=}$ is a matrix of constants, $\underline{\underline{G}}(s)$ is said to be semi-proper. Otherwise $\underline{\underline{G}}(\mathrm{~s})$ is improper (Rosenbrock, 1974). 
For SISO systems Theorem 1 becomes necessary and sufficient.

Theorem 2: Assume $h(s)$ is a proper rational transfer function. $h(s)$ is integral stabilizable if and only if $h(0)>0$.

Proof: This follows immediately from Theorem 7.

Theorem 2 simply states the well known result that positive feedback leads to instability. An SISO system with positive steady state gain can only be controlled with a negative feedback loop. Theorem 1 is a generalization of the negative feedback condition to multivariable systems.

It should be emphasized that Theorems 1 and 2 say nothing for systems where $\stackrel{\mathrm{H}}{=}(\mathrm{s})$ is improper. In this case, even if $\operatorname{det}(\underline{\underline{H}}(0))<0$, there may exist $a k>0$ such that the closed loop system is stable, as illustrated in the following example.

Example 1: Consider controling the SISO system, $g(s)=\frac{-5(s+1)}{2 s+1}$, with a PI controller, $k c(s) / s=k(s+1) / s$. Clearly, $h(s)=\frac{-5(s+1)(s+1)}{2 s+1}$

is improper. The characteristic equation is

$$
(2-5 k) s^{2}+(1-10 k) s-5 k=0
$$

and the system is stable for $0.4<k<\infty$ despite the fact that $h(0)<0$. Stabilizable systems such as the one shown in Example 1, which are stable for gains $\mathrm{k}, 0<\mathrm{k}_{1}<\mathrm{k}<\mathrm{k}_{2}$ will be referred to in this article as conditionally controllable systems. 
Thus, when $\underline{\underline{H}}(\mathrm{~s})$ is improper and $\operatorname{det}(\underline{\underline{H}}(0))<0$, the closed 1oop system of Figure 3 may be conditionally controllable. It is also important to note that Theorem 1 is only necessary. The next example introduces a system with $\operatorname{det}(\underline{\underline{H}}(0))>0$ which is not integral stabilizable.

Example 2: Consider the system

$$
\stackrel{\mathrm{H}}{=} 1=\left[\begin{array}{rr}
-3 & 2 \\
1 & -4
\end{array}\right]
$$

with $\operatorname{det}\left(\mathrm{H}_{1}\right)=10$. The characteristic equation for the closed loop structure shown on Figure 3 is

$$
s^{2}-7 k s+10 k^{2}=0
$$

The closed loop system is clearly unstable for all $k>0$.

Sandell and Athans (1973) have shown that, for integral stabilizability, $\operatorname{det}(\underline{\underline{H}}) \neq 0 . \quad$ Niederlinski (1971) has derived a theorem similar to Theorem 1 which is incorrect as stated. It is restated in the correct form in the following.

Theorem 3: Consider the control system shown in Figure 3 with plant transfer matrix $\underline{G}(s)$ and diagonal compensator $\underline{\underline{C}}(s)$. Let us assume that the following conditions hold:

(a) $\underline{G}(s)$ is stable.

(b) $\stackrel{\mathrm{H}}{=}(\mathrm{s})=\underline{\underline{G}}(\mathrm{~s}) \underline{\underline{\mathrm{C}}}(\mathrm{s})$ is rational and proper.

(c) A11 one-variable control systems, obtained from the multi-variable system by opening any n-1 feedback loops, are stable. 
Then the system is unstable for all $\mathrm{k}>0$ if

$$
\frac{\operatorname{det}(G(0))}{\prod_{i=1}^{n} g_{i i}(0)}<0
$$

Furthermore condition (13) is necessary and sufficient for systems of size smaller or equal to two.

Proof: See Appendix A

It must be observed that in the original statement of the theorem, (13) is claimed to be a necessary and sufficient condition for "structurally monotonic instability". In light of the proofs shown in Appendix A, it is clear that Theorem 3 is a special case of Theorem 1 and that Equation (13) is a sufficient but not necessary condition for instability, except for $2 \times 2$ and SISO systems. $3 \times 3$ and larger systems which satisfy conditions (a)-(c) and violate (13) but which are "structurally monotonic unstable", can easily be constructed. An exar.ple of such systems is shown in Appendix B (Example 14). Furthermore, Niederlinski did not require assumption (b). Example 1 shows that Theorem 3 is incorrect without this assumption.

\section{Failure Sensitivity (Morari, 1983)}

The concepts of sensor and actuator failure are comprehensive and designate actual hardware failure as well as the saturation of a manipulated variable. A burned-out thermocouple, a broken transducer, a stuck valve or the loss of pressure in the line leading to it are examples of failed sensors and actuators. A fully opened valve or a maximum load on an electrical heater 
are examples of saturated variables. Both sensor and actuator failure can have adverse consequences on a control system. The failure of a sensor, for example, means that an erratic electrical signal is sent to the controller. When this happens the integral controller will take action with the aim of eliminating the offset between the received signal and the setpoint. Because the action of the controller is based on an erroneous input signal, it may be tota1ly inappropriate for the system and ultimately lead to instabilities. Actuator failure, on the other hand, brings an end to all control action an equally dangerous situation.

The control problems created by the failure of a sensor or an actuator can be remedied by placing the controller in the failure loop in the off-line mode. In such a situation, it is desirable that without readjustments to the other parts of the control system, system stability be preserved. Sensor and actuator failure sensitivity can be defined rigorously as follows.

Definition 3: The system shown in Figure 4 is j-sensor failure sensitive (j-SFS) if the complete system is integral stabilizable but the reduced system with the $j$ th sensor removed $\left(k_{j}=0\right)$ is not.

To make this definition meaningful we assume that the failure has been recognized and that the loop with the faulty sensor has been taken out of service, i.e., $\mathrm{k}_{\mathrm{j}}$ has been set to zero. The practical implications of this definition are straightforward. If the complete system is integral stabilizable there exists a $k>0$ such that the closed loop system is stable. If the system is $j$ - SFS then the system will become unstable as soon as the $j$ th sensor is removed $\left(k_{j}=0\right)$ regardless of controller tuning, $i . e$, regardless of how $k>0$ was chosen. 
More care has to be used in the definition of actuator failure sensitivity. If only ( $n-1)$ actuators are operating, only (n-1) variables can be controlled in an offset-free manner. Thus any actuator failure requires that one controlled variable be left uncontrolled. For simplicity in notation we will assume that output $y_{j}$ is left uncontrolled when the actuator of $u_{j}$ fails.

Definition 4: The system shown in Figure 4 is j-actuator failure sensitive (j-AFS) if the complete system is integral stabilizable but the reduced system with the $j$ th actuator and the $j$ th sensor removed and with the controller appropriately reduced is not.

The following theorems follow directly from Theorem 1 .

Theorem 4: Assume that $\stackrel{\mathrm{H}}{=}(\mathrm{s})$ is rationa1, proper and integral stabilizable $(\operatorname{det}(\underline{\underline{H}}(0))>0)$. The system is $j-\operatorname{SFS} i f \operatorname{det}\left(\underline{\underline{H}}^{j j}(0)\right)<0$.

Theorem 5: Assume that $\underline{\underline{H}}(\mathrm{~s})$ is rational, proper and integral stabilizable $(\operatorname{det}(\underline{\underline{H}}(0))>0)$. The system is $j-\operatorname{AFS} i f \operatorname{det}\left(\underline{G}^{j j}(0) \underline{\underline{C}}^{j j}(0)\right)<0$.

Summarizing, we can say that if upon removal of an actuator and/or sensor the sign of the determinant of the steady state gain matrix changes, the whole control system has to be redesigned to maintain stability a highly undesirable situation! Thus every effort has to be made to design the compensator $\cong(s)$ such that these problems are avoided. Sensor failure sensitivity can be easily removed by a steady state decoupler $\underline{\underline{C}}=\underline{\underline{G}}(0)^{-1}$. 
Then $\operatorname{det}(\underline{\underline{H}}(0))=1$ and $\operatorname{det}\left(\underline{\underline{H}}^{j j}(0)\right)=1$ and therefore the system is not $j-$ SFS with respect to any sensor $j$. No such simple scheme exists to avoid AFS. Of special interest is $j$-SFS and $j$-AFS when the structure of the the compensator $\underline{\underline{C}}(\mathrm{~s})$ is "decentralized", that is one input-output pair is controlled separately from the rest. It burns out that the RGA provides some information in this respect. Note that in the case of a diagonal $\cong(s)$ j-SFS and j-AFS are equivalent.

Relationship to the Relative Gain Array

Theorem 6: If $\lambda_{j j}(\underline{\underline{G}})<0$ then for any compensator $\underline{\underline{C}}(\mathrm{~s})$ with the properties

(a) $\underline{G}(s) \underline{C}(s)$ is proper.

(b) $c_{j \ell}=c_{2 j}=0 \quad \forall \ell \neq j$

( $y_{j}$ affects $u_{j}$ only, $u_{j}$ is affected by $y_{j}$ only).

and any $k>0$ the closed loop system shown in Figure 4 has at least one of the following properties:

(a) The closed loop system is unstable.

(b) Loop $j$ is unstable by itself, i.e., with all the other loops opened.

(c) The closed loop system is unstable as loop $j$ is removed.

Proof: See Appendix A

This theorem can be interpreted in two ways. Let us assume first that loop $j$ is to be designed independently of the others. Then Theorem 6 implies 
that if loop $j$ by itself is stable and if all the other loops with the loop $j$ removed are stable $((b)$ and (c) are not met) then the closed 1oop system must be unstable. Thus it is impossible to design loop $j$ independently of the others.

On the other hand let us assume that for a particular $\cong(s)$ there exists $a k>0$ such that the closed loop system is stable. Then either 1oop $j$ is unstable by itself or the system becomes unstable when loop $j$ fails (j-SFS/AFS) or both. Thus the system is extremely failure sensitive.

There are two ways around this problem: One could sacrifice the single loop structure of loop $j$, e.g., introduce a steady-state decoupler. This w1ll avoid SFS as was argued previously. The other possibility is to look for an alternate pairing of manipulated and controlled variables. Trivially, because of the properties of the RGA, for $2 \times 2$ systems there is always a pairing such that $\lambda_{11}=\lambda_{22}>0$. However, for $3 \times 3$ and larger systems there might be no pairing for which all the $\lambda_{j j}$ 's are positive, as an example by Koppel referenced by McAvoy (1983) demonstrates.

Example 3: Consider the system transfer matrix

$$
\underline{G}_{1}=\left[\begin{array}{ccc}
1 & 1 & -0.1 \\
0.1 & 2 & -1 \\
-2 & -3 & 1
\end{array}\right]
$$

Using Equation (9) to evaluate the RGA

$$
\Lambda_{1}=\left[\begin{array}{rrr}
-1.89 & 3.59 & -0.7 \\
-0.13 & 3.02 & -1.89 \\
3.02 & -5.61 & 3.59
\end{array}\right]
$$


It is clear that there exists no permutation of the variables such that $\lambda_{j j}>0 ;(j=1,3)$.

We see that not only is the control of MIMO systems very different from that of SISO systems, but $3 \times 3$ systems can have features not found in $2 \times 2$ systems. In $2 \times 2$ systems SFS/AFS can always be avoided even when single 1oop controllers are used as long as the inputs and outputs are paired correctly. On the other hand in some $3 \times 3$ systems SFS/AFS cannot be removed unless multivariable controllers, e.g., steady-state decouplers are used.

Finally, it is worth emphasizing again that negative diagonal relative gains are sufficient but not necessary for the properties of Theorem 6 .

For $3 \times 3$ and larger systems all properties of Theorem 6 might hold even when $\lambda_{i i}>0$. 
Discussion and Conclusions

The key theorem of this section is Theorem 1 which states a necessary condition for integral stabilizability. All the other theorems with the exception of those relating specifically to SISO (Theorem $2 \& 3$ ) and $2 \times 2$ (Theorem 3) systems follow directly from Theorem 1. Also the general interpretation of negative diagonal elements of the RGA in terms of integral stabilizability and AFS/SFS is a direct consequence of Theorem 1 . Indeed, the proper application of Theorem 1 can provide much more insight than the sign of $\lambda_{j j}$ alone. A check of the condition of Theorem 1 shows when a particular system with a particular compensator is not integral stabilizable and when there is a AFS/SFS problem. $\lambda_{j j}<0$ indicates only that at least one of the three properties in Theorem 6 holds. Further considerations have to be used to determine which one.

Some relationship between Niederlinski's theorem, integrity ${ }^{*}$ ) and negative elements in the RGA was postulated for $2 \times 2$ and $3 \times 3$ systems by Gagnepain and Seborg (1982). The general result expressed through Theorem 6 is new. Moreover Niederlinskis (corrected) result (Theorem 3) is only a sufficient condition for instability. If condition (13) is violated nothing can be said about stability. Therefore all that can be concluded from $\lambda_{j j}<0$ is also only sufficient for instability.

The strength of the results in this section is that only a knowledge of the steady state gain matrix is required for making all these conclusions on stability/instability. The detailed system dynamics are irrelevant. The weakness is that only necessary conditions for stability are given. Stability

*) Integrity denotes the ability of a closed-loop system to remain stable under sensor/actuator failure (MacFarlane, 1972). 
cannot be guaranteed, because the conditions are not sufficient. In the next section we will show that much more information can be extracted from the steady state gain matrix and that sufficient conditions for stability can also be obtained. 
INTEGRAL CONTROLLABILITY AND FAILURE TOLERANCE

Our objective is not only to derive conditions on $\underset{\underline{H}}{(0)}$ that guarantee the existence of a positive $\mathrm{k}$ for which the closed loop system in Figure 4 is stable but we would also like to exclude systems which are only conditionally controllable. Conditionally controllable systems are clearly undesirable from a practical point of view. Not only is it difficult to determine the range of gains for which the closed loop system is stable, but this range is likely to change with evolving process operating conditions. In view of this observation, it would be convenient if we required that the closed loop system in Figure 4 remain stable as the controller gain is made arbitrarily sma11. The concepts of integral controllability and fault tolerance are based on this idea (Morari, 1983).

\section{Integral Controllability}

Definition 5: The open-loop stable system $\underline{\underline{H}}(\mathrm{~s})$ is called integral controllable if there exists a $k^{*}>0$ such that the closed 100 p system shown in Figure 4 is stable for all values of $k$ satisfying $0<k \leqslant k^{*}$ and has zero tracking error for asymptotically constant disturbances.

In this definition, the emphasis is placed on the existence of a range of positive gains starting from zero rather than any exact value. A practical consequence of this definition is that integral controllable systems can be tuned on-line starting with a very low gain for which stability is guaranteed, and then increasing the gain until acceptable performance is achieved.

The conditions under which a system $\underline{\underline{H}}(\mathrm{~s})$ is integral controllable are specified in the following theorem. 
Theorem 7: The rational system $\stackrel{\mathrm{H}}{=}(\mathrm{s})$ is integral controllable if all the eigenvalues of $\mathrm{H}(0)$ lie in the open right half complex plane. The rational system $\underline{H}(\mathrm{~s})$ is not integral controllable if any of the eigenvalues of $\underline{\underline{H}}(0)$ lie in the open left half complex plane.

\section{Proof: See Appendix A}

Theorem 7 says nothing about systems for which the eigenvalue of $\underline{\underline{H}}(0)$ lie in the closed right half plane and include eigenvalues on the imaginary axis (not at the origin). This is demonstrated in the next example.

Example 4: Consider the following transfer matrices

$$
\underline{\underline{H}}_{2}(s)=\left[\begin{array}{cc}
0 & -\frac{s+1}{s+10} \\
\frac{s+1}{s+10} & 0
\end{array}\right] ; \quad \stackrel{H}{=}_{3}(s)=\left[\begin{array}{cc}
0 & -\frac{s-1}{s+10} \\
\frac{s-1}{s+10} & 0
\end{array}\right]
$$

The eigenvalues of $\mathrm{H}_{2}(0)$ and $\underline{\mathrm{H}}_{3}(0)$ are $\pm 0.1 \mathrm{i} . \mathrm{H}_{2}(\mathrm{~s})$ is closed-loop stable and $\stackrel{\mathrm{H}}{=}_{3}(\mathrm{~s})$ is closed-1oop unstable for all $\mathrm{k}>0$.

It was shown in Example 1 that a system where $\underset{H}{H}(\mathrm{~s})$ is improper can sometimes be conditionally controllable when $\operatorname{det}(\underline{\underline{H}}(0))<0$. The following Corollary states that such systems are not integral controllable.

Corollary 1: If $\operatorname{det}(\underline{\underline{H}}(0))<0$, then $\underline{\underline{H}}(\mathrm{~s})$ - be it proper or improper - is not integral controllable.

Proof: See Appendix A 
Obviously al1 integral controllable systems are integral stabilizable, but the reverse is not necessarily true. Integral stabilizable systems which are not integral controllable can only occur when an even number of eigenvalues of $\underset{\underline{H}}{(}(0)$ are in the left half plane because a necesaary condition for integral stabilizability is $\operatorname{det}(\stackrel{\mathrm{H}}{=}(0))>0$ (Theorem 1 ). This is illustrated in the next example:

Example 5: Consider the system

$$
{ }_{=}^{H}(s)=\left[\begin{array}{ll}
\frac{-3(-s+1)}{(s+1)(0.5 s+1)} & \frac{4}{(0.5 s+1)} \\
\frac{-4}{(0.5 s+1)} & \frac{2}{(0.5 s+1)}
\end{array}\right]
$$

where $\underline{\mathrm{H}}_{4}(0)$ has both eigenvalues in the left half plane

$$
\begin{aligned}
& Z_{1}(\underline{\underline{H}}(0))=-0.5+3.12 i \\
& Z_{2}(\underline{\underline{H}}(0))=-0.5-3.12 i
\end{aligned}
$$

and is therefore not integral controllable. It can be integral stabilizable however because $\operatorname{det}\left(\mathrm{H}_{4}(0)\right)=10$. Indeed, the Routh test shows the system to be stable for $0.157<\mathrm{k}<0.389 . \mathrm{H}_{4}(\mathrm{~s})$ is therefore conditionally controllable. SISO systems are exceptions.

Theorem 8: Any proper rational system $h(s)$ which is integral stabilizable is also integral controllable.

Proof: The proof follows from that of Theorem 7. 
Integral controllable systems are very desirable in practice. The control loops can be tuned starting from very small gains and unstable closed loop systems can easily be stabilized by decreasing the gain. On the other hand, for systems which are only integral stabilizable, increasing the gain might be necessary for stability and stability might only be maintained for a narrow range of gains.

Using the newly introduced idea of integral controllability it is also possible to strengthen the concept of failure sensitivity.

Failure Tolerance

Definition 6: The system shown in Figure 4 is $j$-sensor failure tolerant $(j-S F T)$ if both the complete system and the reduced system with the $j$ th sensor removed $\left(k_{j}=0\right)$ are integral controllable.

Again we have to assume that the sensor failure has been recognized and that the faulty sensor has been removed from service. $j-S F T$ is a very rich system property. The controller of a j-SFT system can always be tuned such that the closed loop system will remain stable when sensor $j$ fails. After failure all the inputs are used to control the remaining outputs and the control quality might very well degrade, but without any controller adjustments, stability will be preserved.

Just as in the definition of AFS we will assume that output $y_{j}$ is left uncontrolled when the actuator of $u_{j}$ fails. 
Definition 7: The system shown in Figure 4 is j-actuator failure tolerant ( $j-\mathrm{AFT})$ if both the complete system and the reduced system with the $j$ th actuator and the $j$ th sensor removed are integral controllable.

The following theorems which follow directly from Theorem 7 specify the conditions for sensor and actuator failure tolerance.

Theorem 9: The system shown in Figure 4 with $\underset{H}{=}(s)$ rational is $j-S F T$ if all the eigenvalues of $\underline{\underline{H}}(0)$ and $\underline{H}^{j j}(0)$ are in $\underline{C}^{+}$. It is not $j-S F T$ if any of the eigenvalues of $\underline{\underline{H}}(0)$ or $\underline{H}^{j j}(0)$ are in $\underline{\mathrm{C}}^{-}$.

Theorem 10: The system shown in Figure 4 with $\stackrel{\mathrm{H}(\mathrm{s})}{=}$ rational is $\mathrm{j}$-AFT if all the eigenvalues of $\underline{\underline{H}}(0)$ and $\underline{G}^{j j}(0) \underline{G}^{j j}(0)$ are in $\underline{C}^{+}$. It is not $j-A F T$ if any of the eigenvalues of $\underline{\underline{H}}_{\underline{G}}(0)$ or $\underline{G}^{j j}(0) \underline{\underline{C}}^{j j}(0)$ are in $\underline{\mathrm{C}}^{-}$.

A few examples will illustrate these concepts.

Example 6: Let us assume $\underline{\underline{C}}(s)=\underline{\underline{I}}$ and consider the steady state gain matrix

$$
\stackrel{\mathrm{H}}{=}_{5}=\stackrel{\mathrm{G}}{=}_{5}=\left[\begin{array}{ccc}
1 & -2 & -2 \\
0 & 1 & 1 \\
1 & -2 & -1.5
\end{array}\right]
$$

with characteristic equation

$$
-z^{3}+0.5 z^{2}-2 z+0.5=0
$$

Setting $Z=-\mu$ and substituting into Equation (14) yields:

$$
\mu^{3}+0.5 \mu^{2}+2 \mu+0.5=0
$$


Using the Routh criterion, it is easy to show that all three roots of Equation (15) lie in the left-hand complex plane and therefore all three roots of the characteristic equation lie in the right-hand complex plane. The system is therefore integral controllable by virtue of Theorem 7 . If loop 3 were to fail, the system would reduce to

$$
\stackrel{\mathrm{H}}{=}_{5}^{33}=\left[\begin{array}{ll}
1 & -2 \\
0 & 1
\end{array}\right]
$$

and would still be integral controllable since both eigenvalues of $\stackrel{H}{=}_{5}^{33}$ are positive. If loop 1 or loop 2 were to fail, the system would respectively reduce to:

$$
\stackrel{H}{=}_{5}^{I I}=\left[\begin{array}{cc}
1 & 1 \\
-2 & -1.5
\end{array}\right] \text { and } \stackrel{H}{=}_{5}^{22}=\left[\begin{array}{cc}
1 & -2 \\
1 & -1.5
\end{array}\right]
$$

However, since $\stackrel{H}{=}_{5}^{11}$ and $\stackrel{\mathrm{H}}{=}_{5}^{22}$ each have one negative eigenvalue, the reduced system is no longer integral controllable. $\underline{\underline{H}}_{5}$ is therefore only $3-\mathrm{AFT} / \mathrm{SFT}$.

Finally it is worth pointing out that whereas loops 1 and 2 are integral controllable by themselves, loop 3 is not since $h_{33}<0$.

Example 7: Consider now the system

$$
\stackrel{H}{=}_{6}=\stackrel{G}{=}_{6}\left[\begin{array}{ccc}
1 & 20 & 1 / 2 \\
0 & 1 & 1 / 2 \\
1 & 1 & 1
\end{array}\right]
$$

with characteristic equation

$$
-z^{3}+3 z^{2}-2 z+10=0
$$


Substituting for $Z=-\mu$ and using the Routh criterion reveals that some of the eigenvalues of $\stackrel{\mathrm{H}}{=}_{6}$ lie in the left-hand complex plane, implying that the system is not integral controllable. If loop 1 is removed, however, the system reduces to

$$
\underset{=6}{\mathrm{H}}=\left[\begin{array}{cc}
1 & 1 / 2 \\
1 & 1
\end{array}\right]
$$

with positive eigenvalues implying integral controllability. Similar analyses show that any $2 \times 2$ pairing of the variables results in an integral controllable system. Finally, since the diagonal elements of $\stackrel{\mathrm{H}}{=}_{6}$ are all positive, all three loops are integral controllable on an SISO basis.

Relationship to the Relative Gain Array

Except for $2 \times 2$ systems and, to a 1esser extent $3 \times 3$ systems, the RGA gives no information on SFT and AFT.

Theorem 11: Let $\underline{\underline{G}}(\mathrm{~s})$ be a $2 \times 2$ system. If $\lambda_{j j}(\underline{G})>0$ then there exists a diagonal compensator $\underline{\underline{C}}(\mathrm{~s})$ such that $\underline{\underline{H}}(\mathrm{~s})$ is $1-\mathrm{SFT} / \mathrm{AFT}$ and $2-\mathrm{SFT} / \mathrm{AFT}$.

Proof: See Appendix A

In the case of $3 \times 3$ systems a weaker result is available.

Corollary 2: Let $\underline{\underline{G}}(\mathrm{~s})$ be a $3 \times 3$ system with $\lambda_{j j}(\underline{\underline{G}})>0, j=1-3$. If a diagonal compensator $\cong(\mathrm{s})$ can be found such that: 


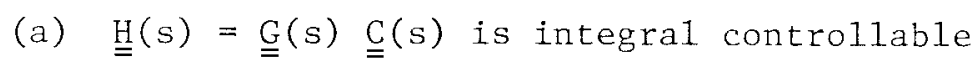

(b) $h_{j j}(0)>0, j=1-3$

then the closed loop system shown in Figure 4 is $j$-SFT/AFT for $j=1-3$.

Proof: See Appendix A

For larger systems the RGA provides no information on SFT/AFT.

Discussion and Conclusions

The results on integral stabilizability and failure sensitivity in the previous section required the sign of the principal minors of the steady state gain matrix $\underset{=}{\mathrm{H}}(0)$ to be checked. In this section we showed that much stronger and much more useful information can be obtained by looking at the eigenvalues of $\mathrm{H}(0)$. We learned that for easy controller tuning the compen-

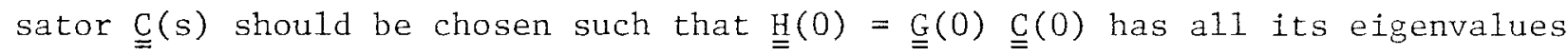
in $\underline{C}^{+}$. For $j-S F T$ the reduced system with the $j$ th row and column removed should also have its eigenvalues in $\underline{C}^{+}$. If $\cong(0)$ is chosen in this manner then we are guaranteed that the controller can be detuned such that the closed loop system will remain stable when the sensor $j$ fails. A similar condition was derived for $j-\mathrm{AFT}$.

C1ear1y $\underline{\mathrm{S}}(0)=\underline{\underline{G}}(0)^{-1}$ will do the trick, but can simpler compensators be found which are equally effective? Theorem 11 shows that $2 \times 2$ systems play a very special role among all multivariable systems. By appropriately pairing the variables to make $\lambda_{j j}>0$ and using just a diagonal compensator (single loop controllers) 1-SFT/AFT and 2-SFT/AFT can always be accomplished. 
Thus for $2 \times 2$ systems the single loop controllers can always be tuned separately. If sufficiently conservative settings are selected, complete failure tolerance is guaranteed. As we pointed out previously, for larger systems it is often not even possible to avoid failure sensitivity with single-loop controllers. These findings and the fact that the majority of multivariable process control problems are $2 \times 2$ might very we11 explain the almost complete absence of multivariable compensators in the process industries. 
RIGHT HALF PLANE ZEROS AND THE RGA

It is well known (Bode, 1945, Shinskey, 1979) that it is difficult to obtain good control for SISO systems with inverse response. Since the initial response is in the opposite direction from the steady state it seems logical that such systems would present control difficulties. It is well established that the inverse response implies and is implied by RHP zeros of the transfer function. It has also been recognized that for multivariable systems the real problem is due to the presence of the "RHP Transmission Zeros" rather than the inverse response which may or may not be observed. If the system $\underline{\underline{G}}(\mathrm{~s})$ is stable its RHP-transmission zeros can be defined as the RHP zeros of det $\underline{G}(s)$. If a system has RHP transmission zeros the quality of control which can be achieved is impaired regardless of how sophisticated a control system is used.

Al1 these issues have been considered in detail by Holt \& Morari (1984). Here we would like to ask if there is any connection between RHP zeros and negative elements in the RGA as has been postulated in the 1iterature. As the following examples show there is no connection between RHP transmission zeros and $\lambda_{j j}<0$.

Example 8: Consider the following transfer matrices:

$$
\begin{aligned}
& \underline{G}_{2}(s)=\frac{1}{s+1}\left[\begin{array}{cc} 
\pm s+2 & 1 \\
3 & 1
\end{array}\right] ; \quad \lambda_{11}=-2, \text { Zeros at } \pm 1 \\
& \underline{G}_{3}(s)=\frac{1}{s+1}\left[\begin{array}{cc} 
\pm s+2 & 1 \\
-3 & 1
\end{array}\right] ; \quad \lambda_{11}=2 / 5 \text {, Zeros } a t \pm 5
\end{aligned}
$$


Thus with $\lambda$ positive or negative the zeros can be either in the RHP or the LHP.

If controllers of arbitrary complexity can be used, RHP transmission zeros are the only factors preventing perfect control. If the controller structure is restricted, for example, to single loops without multivariable compensation, the control quality will degrade even if no RHP transmission zeros are present. It is clear that the degree of degradation depends on the system. It turns out that it can be predicted in some sense by negative elements in the RGA and that it has some connection to RHP zeros (not to RHP transmission zeros).

Theorem 12: Let us denote by $g_{j j, O L}(s)\left(=g_{j j}(s)\right)$ the transfer function between $u_{j}$ and $y_{j}$ when the system is open loop and by $g_{j j, C L}(s)$ the transfer function between $u_{j}$ and $y_{j}$ when all the other inputs and outputs are under integral control. If the following conditions hold:

(a) $g_{j j, O L}(s)$ does not have any RHP zeros.

(b) $\underline{\underline{H}}(\mathrm{~s})$ is strictly proper.

(c) All other elements of $\underline{\underline{G}(s)}$ have equal or higher pole excess than $g_{j j}$ (s). (d) $\lambda_{j j}<0$

then $g_{j j, C L}(s)$ has an odd number of RHP zeros or a pole.

Proof: See Appendix A

The necessity of assumptions (b) and (c) must be emphasized. Examples (14) and (15) in Appendix B show that the theorem breaks down if either of these assumptions is not met. 
Theorem 12 is stated under somewhat different assumptions and without proof in a publication by Bristol (1977). A similar statement is contained in Bristol's original paper (1966). Plausibility arguments for 2x2 systems can be found in McAvoy's book (1983). The general case is proven here for the first time. Interpreting Theorem 12 together with Theorem 6 we can draw the following conclusions for the case that loop $j$ is controlled separately from the others in systems with $\lambda_{j j}<0$. In order to make the closed loop system stable the designer has in general two options:

1) Loop $j$ is stable by itself but the closed loop system is $j$-SFS.

2) The closed loop system is $j-S F T$ but $g_{j j, C L}$ (s) has a RHP zero which causes performance deterioration. In addition loop $j$ is unstable by itself.

Both options are undesirable in practice. Therefore in cases where $\lambda_{j j}<0$ cannot be avoided,1ike in some $3 \times 3$ and larger systems, the use of multivariable compensators which do not leave loop $j$ isolated can be very advantageous both for performance and increased failure tolerance. 
ROBUSTNESS AND THE RELATIVE GAIN ARRAY

Controllers are designed on the basis of inaccurate models and must be tuned such that stability is preserved even, for example, when the system changes due to changes in operating conditions. The ability of a closed loop system to remain stable in the presence of model/plant mismatch will be referred to as robustness. Closed loop systems which become unstable for a "sma11" model/plant mismatch will be called sensitive. In this section we want to determine if the RGA contains any information on system sensitivity or robustness.

The first suspicion would be that if for $\lambda_{j j}>0$ for the model, but $\lambda_{j j}<0$ for the real plant, the closed loop system with the controller designed on the basis of the model would be unstable. This conjecture is incorrect. Let us consider the following examples with $\underline{G}_{p}$ denoting the plant and $\underline{G}_{M}$ the model.

Example 9:

$$
\begin{aligned}
& \underline{G}_{\mathrm{M}}=\left[\begin{array}{ll}
1 & -1 \\
3 & 2
\end{array}\right] ; \lambda_{11}=2 / 5 \\
& \stackrel{\mathrm{G}}{=}=\left[\begin{array}{rr}
-1 & -1 \\
3 & 2
\end{array}\right] ; \lambda_{11}=-2
\end{aligned}
$$

Both eigenvalues of $\underline{G}_{p}$ and $\underline{G}_{M}$ are positive, i.e., $\underline{\underline{G}}_{p}$ and $\underline{G}_{M}$ are integral controllable. Thus despite the fact that $\lambda_{11}$ changes sign, a single controller exists for which $\underline{G}_{p}$ and $\underline{G}_{M}$ will be stable. Obviously, with $\lambda_{11}$ changing sign, the system will become failure sensitive and an RHP zero might be introduced 
causing performance deterioration, but stability can be preserved with a properly tuned controller.

The next example shows that a closed loop system can become unstable even when $\lambda_{11}>0$ for both $\underline{G}_{\mathrm{p}}$ and $\underline{G}_{\mathrm{M}}$.

Example 10:

$$
\begin{aligned}
& \stackrel{G}{G}_{M}=\left[\begin{array}{cc}
1 & -1 \\
3 & 2
\end{array}\right] ; \lambda_{11}=2 / 5 \\
& \stackrel{G}{=}=\left[\begin{array}{rr}
-1 & -1 \\
3 & -2
\end{array}\right] ; \lambda_{11}=2 / 5
\end{aligned}
$$

For $\underline{G}_{p}$ both eigenvalues are in $\underline{C}^{-}$whereas for $\underline{G}_{M}$ they are in $\underline{C}^{+}$. Therefore, in general, there will be no controller for which both $\underline{G}_{\mathrm{p}}$ and $\underline{G}_{M}$ will be stable.

Though apparent1y the RGA cannot be used as a tool to determine when a closed loop system will become unstable if the plant and model do not agree, the example below shows that the RGA might provide some information on when a system is particularly "sensitive".

Examp1e 11:

$$
\begin{aligned}
& \stackrel{\underline{G}}{=}=\left[\begin{array}{ll}
1 & 1.05 \\
0.95 & 1
\end{array}\right] ; \lambda_{11}=400 ; \operatorname{det}\left(\underline{G}_{M}\right)=0.0025 \\
& \stackrel{G}{=}=\left[\begin{array}{ll}
1 & 1.05 \\
1 & 1
\end{array}\right] ; \lambda_{11}=-20 ; \operatorname{det}\left(\underline{G}_{p}\right)=-0.05
\end{aligned}
$$


Here, with one of the plant gains differing by only about $5 \%$ from the model gain, integral stabilizability is lost and obviously there will not exist any controller with which both $\underline{G}_{\mathrm{P}}$ and $\underline{G}_{\mathrm{M}}$ will be stable.

It is noticeable that for this very sensitive system, not only is $\lambda_{11}$ very large, but also a small change in $g_{21}$ induces a large change in $\lambda_{21}$ and $\lambda_{11}$. The relationship between changes in $g_{i j}$ and $\lambda_{i j}$ is easily quantified and leads to the following result.

Theorem 13: Consider the nxn transfer matrix $\underline{G}$ with its inverse $\underline{G}^{-1}$ and its

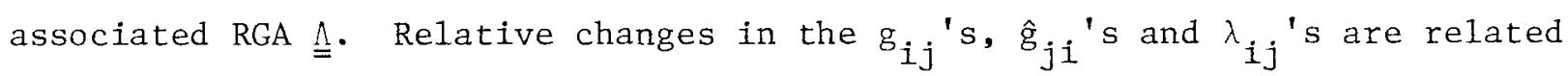
by the following expressions:

$$
\frac{d \lambda_{i j}}{\lambda_{i j}}=\left(1-\lambda_{i j}\right) \frac{d g_{i j}}{g_{i j}}
$$

and

$$
\frac{d \lambda_{i j}}{\lambda_{i j}}=\frac{\lambda_{i j}-1}{\lambda_{i j}} \frac{d \hat{g}_{j i}}{\hat{g}_{j i}}
$$

\section{Proof: See Appendix A.}

(17) shows that as $\lambda_{i j}$ grows in magnitude away from unity, it becomes more sensitive to errors in $g_{i j}$, as Example 11 clearly illustrates. shows that for large $\lambda_{i j}$, relative changes in $\lambda_{i j}$ and $\hat{g}_{j i}$ are approximately equa1. These expressions confirm the suggestion that the RGA might provide some information regarding when a system is particularly "sensitive." Indeed, they show that for large $\lambda_{i j}$, small relative changes in $g_{i j}$ lead to 1 arge relative changes in $\hat{g}_{j i}$, an indication of an error sensitive system. 
These observations will be further substantiated in the next few sections. It will first be shown that "ill-conditioned" matrices display this sensitivity and that the "condition number" can be used as a measure of conditioning. Next, the condition number will be related to system robustness and finally, a relationship between the condition number and the RGA will be derived.

It should be emphasized that the observed sensitivity phenomenon is a characteristic which is specific to MIMO systems and is not found in SISO systems. The sign of the steady state gain of a SISO system has to change, i.e., the gain has to change by more than $100 \%$ in order for the system to lose integral stabilizability. On the other hand, depending on the particular parameter values, a MIMO system can lose integral stabilizability for arbitrarily small changes in the parameters. Such sensitive systems are essentially impossible to control regardless of what single loop or multivariable controller is used. We will show that large elements in the RGA are indicators of "practically uncontrollable" systems.

Condition Number and Matrix Norms

Consider the system of linear equations $\stackrel{A x}{=}=\underline{b}$ where $\underline{A}$ is a nonsingular matrix and $\underline{x}$ is the unknown vector. If it is assumed that matrix $\underline{\underline{A}}$ is subject to perturbations $\underline{\underline{\delta A}}$, then necessarily so is $x$ and we have:

$$
\begin{aligned}
(\underline{\mathrm{x}}+\underline{\delta \mathrm{x}}) & =(\underline{\underline{A}+\underline{\underline{\delta A}}})^{-1} \underline{\mathrm{b}} \\
\underline{\delta \mathrm{x}} & =\left[\left(\underline{\left.\underline{A}+\underline{\underline{\delta A}})^{-1}-\underline{\underline{A}}^{-1}\right] \underline{\mathrm{b}}}\right.\right.
\end{aligned}
$$


Setting $(\underline{\underline{A}}+\underline{\underline{\hat{A}}})=\underline{\underline{B}}$ and using the relationship:

$$
\underline{\underline{B}}^{-1}-\underline{\underline{A}}^{-1}=\underline{\underline{A}}^{-1}[\underline{\underline{A}}-\underline{\underline{B}}] \underline{\underline{B}}^{-1}
$$

it is easy to show that

$$
\begin{aligned}
& \underline{\delta x}=-\underline{A}^{-1}\left(\underline{\underline{\delta A})}(\underline{\underline{A}}+\underline{\underline{\delta A}})^{-1} \underline{\mathrm{b}}\right. \\
& \underline{\delta}=-\underline{A}^{-1}(\underline{\underline{\delta}})(\underline{x}+\underline{\underline{\delta}})
\end{aligned}
$$

Taking norms and using the triangle inequality, the following result is obtained (Forsythe and Moler, 1967);

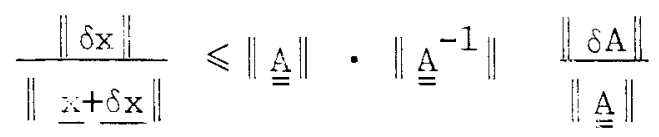

The term $\|\mathrm{A}\| \cdot\left\|\underline{\underline{A}}^{-1}\right\|$ is known as the condition number of $\underline{\underline{A}}$ and is denoted by the letter $\gamma$. It represents the maximum amount by which any relative uncertainty in $\mathrm{A}$ will be amplified and transmitted to the solution x. It is therefore clearly a measure of error sensitivity.

For a given matrix, the condition number will depend on the choice of norm. Rigorously, the norm of a matrix subordinate to a vector norm is defined by:

$$
\|\underline{\underline{A}}\|=\max _{\underline{x} \neq \underline{0}} \underline{\underline{\mathrm{Ax}}} \|
$$

The two norms commonly used in numerical calculations are the 1 - and $\infty$-norms, which are defined as (Johnson \& Riess, 1977): 


$$
\begin{aligned}
& \|\underline{\underline{A}}\|_{1}=\max _{j} \sum_{i=1}^{\mathrm{n}}\left|a_{i j}\right| \\
& \|\underline{\underline{A}}\|_{\infty}=\max _{i} \sum_{j=1}^{\mathrm{n}}\left|a_{i j}\right|
\end{aligned}
$$

Another norm, which has found wide acceptance in process control, is the Euclidean norm. For a matrix A, this is defined as:

$$
\|\stackrel{A}{=}\|_{\mathrm{e}}=\mathrm{Z}_{\max }^{\frac{1}{2}}(\stackrel{\mathrm{A}}{*} \stackrel{\mathrm{A}}{=})=\sigma_{\max }(\stackrel{\mathrm{A}}{=})
$$

where $Z_{\max }(\stackrel{A}{\underline{A}})$ denotes the maximum eigenvalue of $\stackrel{A}{=}$ and $\stackrel{A}{\underline{A}}$ the complex conjugate transpose matrix of $\stackrel{\mathrm{A}}{=}$. The square roots of the eigenvalues of $\stackrel{\mathrm{A}^{*}}{=}$ are called the singular values of $\underline{\underline{A}}$ and are denoted by $\sigma(\underline{\underline{A}})$.

Since

$$
\left\|\underline{\mathrm{A}}^{-1}\right\|_{\mathrm{e}}=\frac{1}{\sigma_{\min } \stackrel{(\mathrm{A})}{=}}
$$

the condition number becomes

$$
\gamma=\|\underline{\underline{A}}\|_{e} \cdot\left\|A^{-1}\right\|_{e}=\frac{\sigma_{\max } \stackrel{(\mathrm{A})}{=}}{\sigma_{\min } \stackrel{(\mathrm{A})}{=}}
$$

where $\sigma_{\max }(\stackrel{A}{=})$ and $\sigma_{\min } \stackrel{(A)}{=}$ represent the maximum and minimum singular values of $\stackrel{\text { A respectively. }}{=}$ 
Robustness and Condition Number

Let us denote the model by $\underline{\underline{G}}(s)$ and the plant which lies in some neighborhood of the model by $\underline{G}(\mathrm{~s})$. We want to find an expression for the maximum allowed difference between $\underline{\underline{G}}(s)$ and $\underline{\underline{G}}(s)$ such that the system remains integral controllable.

Theorem 14: Let us assume that the model $\underline{\underline{G}}(s)$ with the controller $\underline{\underline{C}}(s)$ is integral controllable. Then the plant $\underline{G}(s)$ with the same controller $\underline{\underline{C}}(\mathrm{~s})$ is integral controllable if the model/plant mismatch does not exceed the bound

$$
\frac{\|\underline{\underline{G}-\tilde{G}}\|}{\|\tilde{\underline{G}}\|}<\frac{1}{\|\tilde{\underline{G}}\|\left\|\tilde{\underline{G}}^{-1}\right\|}=\frac{1}{\gamma(\underline{\underline{G}})}
$$

Proof: See Appendix A.

The Condition Number Inequality(26) states that the maximum relative steady state gain error must be less than the inverse of the condition number of $\tilde{G}$. Under these conditions, an integral controller can be found which guarantees the closed-loop stability of the system for both the model $\tilde{G}(s)$ and the plant $\underline{\underline{G}}(s)$. It is important to emphasize that (26) guarantees the existence of such controller but says nothing about the control quality which might be quite poor if the model error is large.

Another problem with (26) is that it depends on the scaling of the inputs and outputs. This is unsatisfactory since whether a system is stable or not must obviously be independent of the scaling. This is demonstrated by the following example. 
Example 12: Consider the transfer matrix:

$$
\underline{\underline{G}}=\left[\begin{array}{ll}
1 & 0 \\
0 & 1000
\end{array}\right]
$$

with

$$
\|\tilde{\underline{G}}\|=1000
$$

and

$$
\gamma(\underline{\underline{\tilde{G}}})=1000
$$

Equation (26) yields:

$$
\|\underline{\underline{G}}-\tilde{G}\|<1 \text {. }
$$

If

$$
\underline{\underline{G}}=\left[\begin{array}{ll}
1+\varepsilon_{1} & 0 \\
0 & 1000+\varepsilon_{2}
\end{array}\right],
$$

then the errors satisfying (27) are, for example:

$$
\left|\varepsilon_{1}\right|<1, \quad \varepsilon_{2}=0
$$

or

$$
\varepsilon_{1}=0,\left|\varepsilon_{2}\right|<1
$$

where (28) is reasonable but (29) is obviously very conservative. Let us next rescale input 2 or output 2 by $1 / 1000$ such that we obtain the scaled model:

$$
\underline{G}^{\prime}=\left[\begin{array}{ll}
1 & 0 \\
0 & 1
\end{array}\right]
$$

with

$$
\left\|\underline{\underline{G}}^{\prime}\right\|=1
$$

and

$$
\gamma\left(\underline{\underline{G}}^{\prime}\right)=1
$$


Equation (26) now yields

$$
\| \underline{\underline{G}-\underline{G}^{\prime} \|<1}
$$

which is much more meaningful because it implies that a $100 \%$ error is allowed in each diagona1 element.

Since the Condition Number Inequality (26) holds for any input/output scaling of the system, it seems appropriate that it should be scaled in the least conservative manner.

Remark 1: The condition number inequality (26) is a useful measure of sensitivity only when the inputs/outputs have been scaled to minimize the condition number.

Justification: See Appendix A.

Unfortunately no general scaling procedure exists which minimizes $\gamma$.

\section{Condition Number and the Relative Gain Array}

The search for a relationship between the condition number and the RGA of a process transfer matrix is spurred by the following observations.

- The condition number is rigorously related to system sensitivity and robustness but is scale dependent.

- A relationship between the RGA and sensitivity has only been demonstrated empirically, but the RGA has the advantage of being scale independent. 
- The elements of the RGA as expressed by Equation (9) and the condition number as defined by Equation (25) display a striking mathematical resemblance. Indeed,

$$
\begin{aligned}
\lambda_{i j} & =g_{i j} \cdot \hat{g}_{j i} \\
\gamma & =\|\underline{\underline{G}}\| \cdot\left\|\underline{G}^{-1}\right\|
\end{aligned}
$$

where the $\hat{\mathrm{g}}_{i j}$ 's are the elements of $\underline{\underline{G}}^{-1}$.

In what follows, it will be shown that the condition number can be related to the RGA: This will show that the RGA is itself a measure of error sensitivity, a result which has been argued for in the past (Bristol, 1966; Jafarey \& McAvoy, 1978; Shinskey, 1979; McAvoy, 1983), but which was never rigorously proved. Results will first be demonstrated for $2 \times 2$ systems.

A difficulty in attempting to link the RGA to the condition number is the fact that whereas the RGA is scale independent (Property 3), the condition number is not. The latter is therefore a function of the units of the transfer matrix $\underline{G}$. This problem can be circumvented by scaling the transfer matrix $\underline{\underline{G}}$ with diagonal matrices in such a way that a minimum or "optimal" condition number is obtained. Optimal scaling simply ensures that the least conservative value of the condition number is obtained and it should not be given a physical interpretation. In the subsequent developments, the condition number is always defined in terms of the Euclidean norm unless otherwise noted. 
Theorem 15: For a $2 \times 2$ transfer matrix $\underline{\underline{G}}$, the minimum condition number $\gamma^{*}$ is given by:

$$
\gamma^{*}=\frac{\left\|\Lambda_{1}\right\|_{1}}{2}\left[1+\sqrt{\frac{\| \Lambda_{1}-1}{\|\underline{\Lambda}\|_{1}+1}}\right]^{2}
$$

Fur thermore:

$$
\begin{aligned}
& \gamma^{*}=1 \text { if and on1y if }\|\Lambda\|_{1}=1 \\
& \gamma^{*} \leqslant 2\|\Lambda\|_{1}
\end{aligned}
$$

and

$$
\gamma^{*}=2\|\Lambda\|_{1} \text { as }\|\Lambda\|_{1} \rightarrow \infty
$$

where $\|\Lambda\|_{1}$ denotes the 1 -norm of the RGA.

Proof: See Appendix A

Note that for $2 \times 2$ systems $\|\Lambda\|_{1}=\|\Lambda\|_{\infty}$, so that Theorem 15 could as well have been expressed in terms of the $\infty$-norm. For convenience, both (31) and (33) are shown in Figure 5. Equation (33) is seen as an excellent approximation of (31) for values of $\| \Lambda_{1}$ greater than 3 .

Several important conclusions can be drawn from Theorem 15. Diagonal and triangular systems as well as systems with an odd number of negative elements (see Property 5) have $\gamma^{*}=\|\triangleq\|_{1}=1$. Therefore they are well behaved and have no sensitivity problems. Even when these systems exhibit "strong" interaction $\left(\lambda_{i i}=0.5\right)$ good control can be obtained despite modeling errors, 
at least when some kind of multivariable compensation, e.g., steady state decoupling, is employed.

$\gamma^{*}$ is bounded above by $2 \| \Lambda_{1}^{\|}$and approaches $2\left\|\Lambda_{1}\right\|$ as it becomes large. Therefore large elements in the RGA imply that $\|\Lambda\|_{1}$ is large which in turn implies that $\gamma^{*}$ is large. Thus systems with $\left|\lambda_{j j}\right|$ large are sensitive to modeling errors and are difficult to control regardless of how sophisticated a control strategy might be used. Systems with $\left|\lambda_{j j}\right|$ large are "practically uncontrollable".

It is important to mention that these results are fully consistent with those of Shinskey (1979). In his investigation of the stability of decoupled $2 \times 2$ systems, Shinskey derived a relationship between $\lambda_{11}$ and the error sensitivity of the decoupler. For systems with $\lambda_{11}<0$ or $\lambda_{11}>1$, the decoupler error required to destabilize the closed loop system was shown to decrease with increasing $\left|\lambda_{11}\right|$. Alternatively, the closed loop stability of systems with $0<\lambda_{11}<1$ was found to be insensitive to decoupler error.

The significance of our results is that not only have we confirmed the general trend of "large" RGA-high sensitivity, we have derived a quantitative relationship (31) between $\|\Lambda\|_{1}$ and the maximum error between plant and model for which closed loop stability can be guaranteed.

The intractibility of the algebra has made it impossible to obtain analytical results equivalent to those of Theorem 15 for systems with more than two inputs/outputs. Some results based on numerical methods are nevertheless available.

Conjecture 1: For the nxn transfer matrix $\underline{\underline{G}}$ the minimum condition number $\gamma^{*}$ is bounded by 


$$
\gamma^{*} \leqslant 2 \max \left[\|\Lambda\|_{1},\|\Lambda\|_{\infty}\right]
$$

Our approach in developing this conjecture was "brute force". We generated a large number of 3 and 4 input/output transfer matrices with random coefficients uniformly distributed between -100 and +100 . Rosenbrock's numerical optimization technique (Rosenbrock, 1960) was then used to obtain the optimal scaling parameters and condition number. Figure 6 shows the relationship that was obtained for $3 \times 3$ matrices when the minimal condition number was plotted against the 1 -norm of the RGA. For $3 \times 3$ systems, our numerical results showed that the 1 - and $\infty$-norms of the RGA were always equal. Likewise, Figure 7 shows the relationship that was obtained for $4 \times 4$ systems when the minimal condition number was plotted against the maximum of the $1-$ and ${ }_{\infty}^{\infty}$-norms of the RGA. Numerical results seldom showed equality between these two norms.

At this point, it must be said that some numerical difficulties were experienced in obtaining optimal scaling parameters for those transfer matrices with two nearly identical singular values or with a singular value very close to zero. These difficulties account for the offline points in Figures 6 and 7 . In spite of this, the relationships shown on these figures show a striking similarity with that shown on Figure 5 for $2 x 2$ systems. This resemblance led us to postulate the validity of Equation (34) for systems of order higher than 4. Finally, it is worth mentioning that Equation (34) was found to hold when the condition number was defined in terms of the 1 -norm or the $\infty$-norm as well.

The results of Theorem 15 and Conjecture 1 are not without substantial practical consequences. They imply that the RGA is itself a measure of 
sensitivity. The determination of a system's sensitivity is therefore reduced from a tedious optimization problem (finding $\gamma^{*}$ ) to a trivial exercise of arithmetic (finding $\|\Lambda\|_{1}$ and $\|\underline{=}\|_{\infty}$ ).

Further considerations led us to establish conditions under which the optimal condition number of a matrix will take on a value of unity. It was seen that for a $2 \times 2$ matrix, this situation will arise if and only if the 1-norm of the RGA is unity.

Theorem 16: No system larger than $3 \times 3$ with all non-zero entries can have a minimum condition number of unity.

Proof: See Appendix A

This theorem implies that as transfer matrices grow in size their optimal condition number grows in magnitude and they generally become more sensitive to modeling errors. 


\section{CONCLUSIONS}

Based on the assumptions that the open loop nxn system $\underline{\underline{G}}(\mathrm{~s})$ is stable and equipped with a controller with integral action, we have shown that a wealth of closed loop information can be extracted from the steady state gain matrix $\underline{\underline{G}}(0)$ alone. Referring to Figure 4, let $\underline{\underline{\mathrm{C}}}(\mathrm{s})$ represent the part of the compensator without the integrator and assume $k>0$. Defining $\underline{\underline{H}}(s)=\underline{G}(s) \underline{\underline{C}}(s)$, we can sumarize the results qualitatively (without restating al1 the assumptions) as follows.

Integral Stabilizability

1) The closed loop system is unstable for all $k>0$ ("non-integral-stabilizable") when $\operatorname{det}(\underline{\underline{H}}(0))<0$.

2) Upon failure of sensor/actuator $j$ the closed loop system becomes unstable ("j-sensor/actuator failure sensitive") when

$\operatorname{det}\left(\underline{\underline{H}}^{j \mathrm{j}}(0)\right)<0 / \operatorname{det}\left(\underline{G}^{j j}(0) \underline{\underline{G}}^{j \mathrm{j}}(0)\right)<0$.

These sufficient conditions for instability are complemented by the following sufficient conditions for stability.

\section{Integral Controllability}

1) The closed loop system is stable for $0<k \leqslant k^{*}$ ("integral controllable") when all the eigenvalues of $\stackrel{\mathrm{H}}{=}(0)$ are in $\underline{\mathrm{C}}^{+}$.

2) There exists a $k>0$ such that the closed loop system remains stable upon failure of sensor/actuator $j$ when all the eigenvalues of both $\stackrel{\mathrm{H}}{=}(0)$ and $\underline{G}^{j \mathrm{j}}(0) \stackrel{\mathrm{C}}{\mathrm{j}}^{j \mathrm{j}}(0)$ are in $\underline{\mathrm{C}}^{+}$.

Integral controllable systems are desirable in F can be tuned on-line starting with a very low gain, $f c$ guaranteed, and then increasing the gain until acceptat achieved. 
With regard to the RGA the following results were established (again the structure of Figure 4 is assumed):

RGA

1) If a single loop controller is used on an input/output pair associated with a negative RGA element, then assuming that the multivariable closed loop system is stable at least one of the following is true:

a) The single loop transfer function includes an RHP zero and the loop is unstable by itself.

b) The overall system becomes unstable when the single loop is opened.

2) For large norms of the RGA the norm of the allowed modelling error $\stackrel{G}{=}-\tilde{G}$ for which closed loop stability is preserved is given approximately by

$$
\| \underset{G-\tilde{G} \|}{\|\underline{\underline{G}}\|} \leqslant \frac{1}{2 \max \left(\|\underline{=}\|_{1},\left\|\Lambda_{\infty}\right\|_{\infty}\right)}
$$

3) Let $\underline{G}(s)$ be a $2 \times 2$ system with $\lambda_{11}>0$ then there exists a diagonal compensator $\underline{\underline{C}}(\mathrm{~s})$ (i.e.,pair of single loop controllers) such that the closed loop system remains stable upon failure of either actuator/sensor.

4) Let $\underset{\underline{G}}{(\mathrm{~s})}$ be a $3 \times 3$ system with $\lambda_{j j}>0 j=1,3$. If a diagona1 compensator $\stackrel{\mathrm{C}}{\underline{\mathrm{C}}} \mathrm{s})$ can be found such that

a) $\quad \underline{\underline{H}}(s)=\underline{\underline{G}}(s) \underline{\underline{C}}(s)$ is integral controllable

b) $h_{j j}(0)>0 j=1,3$

then the closed loop system shown in Figure 4 can be designed such that it is stable upon failure of any sensor or actuator. 
These theoretical findings have the following practical implications.

1) If the system is $2 \times 2$, if the performance specifications are not very strict (no offset, stability in the event of sensor/actuator failure), and if the RGA elements are not very large (preferabiy between 0 and 1), then two single loop controllers without multivariable compensation are satisfactory.

2) If the system is $3 \times 3$ and larger and if negative elements on the diagona1 of the RGA cannot be avoided, a series of single loop controllers will result in both poor performance and low integrity. Multivariable compensation should be used.

3) Large RGA elements are an indication of high sensitivity to modeling error. Systems for which the norm of the RGA is "large" are "practically uncontrollable" regardless of how sophisticated a multivariable controller is employed.

The proof of all theorems relied on the assumption that $\underline{\underline{G}}(\mathrm{~s})$ is rational. However, because the derived conditions involve properties of $\underline{\underline{G}}(0)$ only, the theorems hold also for systems with time delays -- at least in an "engineering sense". 
APPENDIX A

Proof of Theorem 1:

The proof is based on the Routh test. The characteristic equation (CE) for the closed loop system of Figure 4 is given by:

$$
\phi(s) \cdot \operatorname{det}\left(\underline{\underline{I}}+\underline{\underline{H}}(\mathrm{~s}) \frac{k I}{\mathrm{~s}}\right)=0
$$

where $\phi(s)$ is the open loop characteristic polynomial of $\underline{H}(s)$. Express $\underline{H}(s)$ as $\underline{\underline{H}}(\mathrm{~s})=\underline{N}(\mathrm{~s}) \mathrm{d}^{-1}(\mathrm{~s})$ where $\mathrm{d}(\mathrm{s})$ is the common denominator of the elements of $\underline{\mathrm{H}}(\mathrm{s})$ and $\mathrm{N}(\mathrm{s})$ is a polynomial matrix. Equation (A1) can then be expressed as:

$$
\frac{\phi(s)}{\mathrm{sd}(\mathrm{s})} \cdot \operatorname{det}(\mathrm{sd}(\mathrm{s}) \stackrel{\mathrm{I}}{=}+\mathrm{k} \stackrel{\mathrm{N}}{=}(\mathrm{s}))=0
$$

Upon expansion of the determinant, this expression becomes

$$
\frac{\phi(s)}{s d(s)} \cdot\left(s^{n} d^{n}(s)+\ldots+k^{n} \operatorname{det} \stackrel{N}{=}(0)\right)=0
$$

If $\underset{H}{\mathrm{H}}(\mathrm{s})$ is proper, the coefficient of the highest power of $s$ in (A3)will be the coefficient of the highest power of $s$ in $d(s)$. This coefficeint will be positive because of the stability assumption. The closed loop system will be stable only if all the coefficients in $\operatorname{det}(\mathrm{sd}(\mathrm{s}) \underline{\underline{I}}+\mathrm{kN}(\mathrm{s})$ ) are positive. The constant coefficient is $\operatorname{det}(\mathrm{kN}(0))$ and therefore for closed loop stability it is required that $\operatorname{det}(\stackrel{\mathrm{N}}{=}(0))>0$ and $\operatorname{det}(\underline{\mathrm{H}}(0))>0 . \quad$ QED 
Proof of Theorem 3:

We assume that the compensator is diagonal

$$
\stackrel{C}{=}(s)=\operatorname{diag}\left(c_{1}(s), \ldots c_{n}(s)\right)
$$

and that each loop by itself is stable. From Theorem 2 we know that this is the case if and only if

$$
c_{i}(0) g_{i i}(0)>0 \quad \nabla_{i}
$$

or

$$
\prod_{i=1}^{\pi} c_{i}(0) g_{i i}(0)>0
$$

It follows from Theorem 1 that $\stackrel{\mathrm{H}}{=}(\mathrm{s})$ is not integral stabilizable if

$$
\operatorname{det} \underline{\underline{H}}(0)=\prod_{i=1}^{n} c_{i}(0) \operatorname{det} \underline{\underline{G}}(0)<0
$$

From (A4) this is equivalent to Niederlinskis condition

$$
\frac{\operatorname{det} G(0)}{\prod_{i=1}^{n} g_{i i}(0)}<0
$$

In the case of $2 \times 2$ and SISO systems, Equation (13) is also a necessary condition for "structural monotonic instability". This can be proved by showing that if Equation (13) is violated, then there will always exist a compensator $\underline{\underline{C}}(\mathrm{~s})$ and a gain $\mathrm{k}>0$ such that the closed loop system is stable. In the case of $2 \times 2$ systems, let 


$$
\frac{\operatorname{det}(\underline{\underline{G}}(0))}{g_{11}(0) g_{22}(0)}>0
$$

then there always exists a compensator $\underline{\underline{C}}(\mathrm{~s})$ such that $\operatorname{det}(\underline{\underline{H}}(0))>0$, $h_{11}(0)>0$ and $h_{22}(0)>0$.

Indeed, if

$$
\begin{aligned}
& \operatorname{det}(\underline{\underline{G}}(0))>0, g_{11}(0)>0 \text { and } g_{22}(0)>0 \text {, choose } c_{1}(0)>0, c_{2}(0)>0 \\
& \operatorname{det}(\underline{G}(0))>0, g_{11}(0)<0 \text { and } g_{22}(0)<0 \text {, choose } c_{1}(0)<0, c_{2}(0)<0 \\
& \operatorname{det}(\underline{G}(0))<0, g_{11}(0)>0 \text { and } g_{22}(0)<0 \text {, choose } c_{1}(0)>0, c_{2}(0)<0 \\
& \text { If } \operatorname{det}(\underline{\underline{H}}(0))>0 \text { and } h_{11}(0)+h_{22}(0)>0 \text {, the eigenvalues of } \underline{\underline{H}}(0) \text { are }
\end{aligned}
$$
in $\mathrm{C}^{+}$and $\mathrm{a} \mathrm{k}>0$ exists by virtue of Theorem 7 . Finally, in the case of SISO systems, Equation (13) yields:

$$
\frac{\operatorname{det} g(0)}{g(0)}=1>0
$$

This simply expresses the fact that an SISO system is always integral controllable provided the sign of the compensator $\mathrm{c}(\mathrm{s})$ is chosen such that $g(0) c(0)>0$ 
Proof of Theorem 6:

Because $\lambda_{i j}$ is invariant under input and output scaling we have for

any diagonal pre- or post-compensator $\stackrel{C}{\cong}(0)$

$$
\begin{aligned}
\lambda_{i j} & =(-1)^{i+j} g_{i j} \frac{\operatorname{det}\left(G(0)^{j i}\right)}{\operatorname{det}(\underline{G}(0))} \\
& =(-1)^{i+j} h_{i j} \frac{\operatorname{det}\left(H(0)^{j i}\right)}{\operatorname{det}(\underline{\underline{H}(0))}}
\end{aligned}
$$

If $\lambda_{j j}<0$ then one or three of the terms in Equation (A7) is negative. For property (a) $\operatorname{det}(\underline{\underline{H}}(0))<0$; for property (b) $h_{j j}<0$; for property (c) $\operatorname{det}\left(\underline{\mathrm{H}}^{\mathrm{j}} \mathrm{j}(0)\right)<0$. 
Proof of Theorem 7:

Let the Nyquist D-contour be indented at the origin to the right to exclude the pole of $1 / \mathrm{s} \stackrel{\mathrm{H}}{=}(\mathrm{s})$ at the origin. The system will be closed loop stable if none of the characteristic loci (CL) encircles the point $(-1 / k, 0)$. For integral controllability it is necessary and sufficient that the CL intersect the negative real axis only at finite values. An intersection at $(-\infty, 0)$ could only occur because of the pole of $1 / s \underline{H}(s)$ at the origin. Along the indentation, the small semi-circle with radius $\varepsilon$ around the origin, the CL can be described by

$$
\left.Z_{j} \stackrel{(H}{=}(0)\right) \cdot \frac{1}{\varepsilon} e^{-i \phi} \quad-\frac{\pi}{2} \leqslant \phi \leqslant \frac{\pi}{2} ; j=1, n
$$

for small $\varepsilon$. Let $\left.\mathrm{Z}_{j} \stackrel{(\mathrm{H}}{=}(0)\right)=\mathrm{r}_{j} e^{i \ominus}$ then the expression for the CL can be rewritten as

$$
\begin{aligned}
\frac{r_{j}}{\varepsilon} e^{i\left(\Theta_{j}-\phi\right)}-\frac{\pi}{2} & \leqslant \phi \leqslant \frac{\pi}{2} \\
j & =1, n
\end{aligned}
$$

The CL do not cross the negative real axis if $-\pi<\theta_{j}-\phi<\pi$ or $-\frac{\pi}{2}<\theta_{j}<\frac{\pi}{2}$ which means $Z_{j}(\underline{H}(0)) \varepsilon \underline{C}^{+}, j=1, n$. The characteristic 1ocus $j$ crosses the negative real axis if $\frac{\pi}{2}<\theta_{j}<\frac{3 \pi}{2}$ which means $z_{j}(H(0)) \varepsilon \underline{C}^{-}$. Nothing can be said from this proof about systems for which the spectrum of $\underline{\underline{H}}(0)$ is constrained to the closed right half plane and includes eigenvalues on the imaginary axis. 
Proof of Theorem 11:

The necessity follows from Theorem 6. The sufficiency can be proved as follows:

$$
\lambda_{11}=\frac{h_{11} h_{22}}{\operatorname{det}(\underline{\underline{H}}(0))}
$$

There always exists a diagonal compensator $\underline{\underline{C}}$ such that $h_{11}>0(2-\mathrm{SFT} / \mathrm{AFT})$ and $h_{22}>0(1-\mathrm{SFT} / \mathrm{AFT})$. Therefore $\lambda_{11}>0$ implies $\operatorname{det}(\underline{\underline{H}}(0))>0$. The eigenvalues of $\stackrel{H}{=}(0)$ are the roots of

$$
\mathrm{z}^{2}-\left(\mathrm{h}_{11}+\mathrm{h}_{22}\right) \mathrm{z}+\operatorname{det}(\underline{\mathrm{H}}(0))=0
$$

For this second order polynomial $\operatorname{det}(\underline{\underline{H}}(0))>0$ and $h_{11}+h_{22}>0$ implies that a11 the eigenvalues of $\underline{\underline{H}}(0)$ are in the $\mathrm{RHP}$. $\underline{\underline{H}}(\mathrm{~s})$ is therefore integral controllable. Moreover, when $\lambda_{11}<0$ define

$$
\underline{G}^{\prime}=\underline{G}\left[\begin{array}{ll}
0 & 1 \\
1 & 0
\end{array}\right]
$$

that is, exchange the system inputs. Then

$$
\lambda_{11}\left(\underline{G}^{\prime}\right)=\lambda_{12} \stackrel{(G)}{=}=1-\lambda_{11}(\underline{G})>0 \quad \underline{Q E D}
$$


Proof of Theorem 12:

The theorem was stated for $i=j$ but is in fact valid for a11 $i, j=1, n$. Consider the nxn rational transfer matrix $\underline{\underline{G}}(s)$ and assume presently that $g_{i j}(0)>0$. If $\lambda_{i j}<0$, then by virtue of Theorem $6, g_{i j, C L}(s)$ may or may not be stable. In the latter case, clearly it has a pole in $\underline{C}^{+}$. Alternatively, if $g_{i j, C L}(s)$ is stable, the initial and final value theorems can be used to show that it has an odd number of RHP zeros. $g_{i j, C L}(s)$ can be expressed as follows:

$$
g_{i j, C L}(s)=y_{i} /\left.u_{j}\right|_{C L}=g_{i j, O L}(s)-\underline{G}^{i}(s)\left[\left(\underline{\underline{I}}+\underline{\underline{K}}(s) \underline{G}^{i j}(s)\right]^{-I} \underline{\underline{K}}(s) \underline{G}^{j}(s)\right.
$$

where $\quad G^{i}(s)$ is the ith row of $\underline{g}(s)$ less $g_{i j}(s)$

$$
\begin{aligned}
& \underline{G}^{j}(s) \text { is the } j \text { th column of } \underline{\underline{G}}(s) \text { less } g_{i j}(s) \\
& \underline{=}(s) \text { is an }(n-1) \times(n-1) \text { matrix of integral controllers }
\end{aligned}
$$

Observe that Equation (A8) is simply the generalization of Equation (2). Applying a forcing function $u_{j}=1 / s$, the initial value theorem shows that the response has a positive initial slope:

$$
\lim _{t \rightarrow 0} \frac{\partial y_{i}}{\partial t}=\lim _{s \rightarrow \infty} s\left[g_{i j, O L}(s)-\underline{\underline{G}}^{i}(s)\left[\underline{\underline{I}}+\underline{\underline{K}}(s) \underline{\underline{G}}^{i j}(s)\right]^{-1} \underline{\underline{K}}(s) \underline{G}^{j}(s)\right]
$$

Because of assumptions (b) and (c) in Theorem 12, and barring any cancellation in the polynomial, the term

$$
\underline{\underline{G}}^{i}(s)\left[\underline{\underline{I}}+\underline{\underline{K}}(s) \underline{\underline{G}}^{i j}(s)\right]^{-1} \underline{\underline{K}}(\mathrm{~s}) \underline{G}^{j}(s)
$$

will be several orders of magnitude smaller than $g_{i j, \text { OL }}$ (s) for "large" values of s. Accordingly, Equation (A9) reduces to: 


$$
\operatorname{Lim}_{t \rightarrow 0} \frac{\partial y_{i}}{\partial t}=\operatorname{Lim}_{s \rightarrow \infty} s g_{i j, O L}(s)>0
$$

Likewise, the final value theorem shows that

$$
\operatorname{Lim}_{t \rightarrow \infty} y_{i}=\operatorname{Lim}_{s \rightarrow 0} g_{i j, C L}(s)=\frac{g_{i j, O L}(0)}{\lambda_{i j}} \lessdot 0
$$

The positive initial slope (Equation $A 10$ ) and the final negative value (Equation Al1) for $y_{i}$ imply the presence of an odd number of RHP zeros in $g_{i j, C L}(s)$. An analogous result is obtained if it is assumed that $g_{i j, O L}(0)<0$.
$\underline{Q E D}$ 
Proof of Theorem 13:

The relative gain between $u_{j}$ and $y_{i}$ can be expressed as:

$$
\lambda_{i j}=g_{i j} \cdot \hat{g}_{j i}=\frac{g_{i j}}{g_{i j}-\underline{G}^{i}\left(\underline{\underline{G}}^{i j}\right)^{-1} \underline{G}^{j}}
$$

where $\underline{G}^{i}$ and $\underline{G}^{j}$ are defined as in (A8). Differentiation of (Al2) yields (17). (18) is derived analogous1y.

Proof of Theorem 14:

Consider the closed-loop transfer matrix of the system shown in Figure 4 with plant $\underline{\underline{G}}(\mathrm{~s})$ and controller $\underline{\underline{K}}(\mathrm{~s})=\underline{\underline{C}}(\mathrm{~s}) \frac{\mathrm{k}}{\mathrm{s}} \mathrm{I}$,

$$
\underline{\underline{G}}(\mathrm{~s})(\underline{\underline{I}}+\underline{\underline{K}}(\mathrm{~s}) \underline{\underline{G}}(\mathrm{~s}))^{-1} \underline{\underline{K}}(\mathrm{~s})
$$

Letting $\tilde{\underline{G}}(\mathrm{~s})$ denote the process model, (A13) can be shown to be equivalent to the following expression.

$$
\underline{\underline{G}}(\mathrm{~s})\left(\underline{\underline{\mathrm{I}}}+(\underline{\underline{I}}+\underline{\underline{K}}(\mathrm{~s}) \underline{\underline{G}}(\mathrm{~s}))^{-1} \underline{\underline{K}}(\mathrm{~s})(\underline{\underline{G}}(\mathrm{~s})-\underline{\underline{\underline{G}}}(\mathrm{~s}))\right)^{-1}(\underline{\underline{I}}+\underline{\underline{K}}(\mathrm{~s}) \underline{\underline{\underline{G}}}(\mathrm{~s}))^{-1} \underline{\underline{K}}(\mathrm{~s})
$$

Inspection of (A14) shows that the system with the plant $\underline{\underline{G}}(\mathrm{~s})$ will be integral controllable if the following conditions are met:

(1) The nominal system $\underset{\underline{G}}{\underline{G}}(s) \underline{\underline{C}}(s)$ is Integral Controllable.

(2) The transfer matrix

$$
(\underline{\underline{I}}+\underline{\underline{K}}(s) \underline{\underline{G}}(s))^{-1} \underline{\underline{K}}(s)(\underline{\underline{G}}(s)-\underline{\underline{G}}(s))
$$

is integral controllable in the sense of Definition 5.

Condition ( 1 ) is satisfied by hypothesis and therefore all that is needed to prove the theorem is a sufficient condition for the Integral Controllability of (A15). Applying the Sma11 Gain Theorem to (A15), such a condition can be derived by inspection. For finite values of $s$ along the Nyquist D-contour, (A15) can be made arbitrarily small be decreasing $k$. (A15), however, takes on a constant value of 


$$
\underline{\underline{G}}^{-1}(\underline{\underline{G}}-\underline{\underline{G}})
$$

when s contours the pole at the origin, and this is irrespective of how $k$ and $\stackrel{\underline{C}}{(s)}$ are chosen. Thus, in order to ensure that a $k^{*}$ and $a \stackrel{C}{\underline{C}}(\mathrm{~s})$ can be found such that the CL of (A15) do not encircle the point $(-1,0)$ for all values of $k$ satisfying $0<k \leqslant k^{*}$, it is sufficent to require that the eigenvalues of (A16) have a magnitude less than unity. This condition will be satisfied if

$$
\left\|\underline{\tilde{G}}^{-1}(\underline{\underline{G}}-\tilde{\underline{G}})\right\|<1
$$

(A17), in turn, is implied by:

$$
\frac{\|\underline{\underline{G}}-\underline{\underline{G}}\|}{\|\tilde{\underline{G}}\|} \cdot\|\underline{\underline{G}}\| \cdot\left\|\underline{\tilde{G}}^{-1}\right\|<1
$$

or

$$
\frac{\|\underline{G}-\underline{\underline{G}}\|}{\|\underline{\underline{G}}\|}<\frac{1}{\gamma(\underline{\underline{G}})}
$$


Proof of Theorem 15:

We consider the case of a $2 \times 2$ transfer matrix $\underline{G}$. Differential calculus shows that $\mathrm{G}$ will be optimally scaled when pre-and post-multiplied by diagonal matrices $\underline{\underline{S}}_{1}$ and $\underline{\underline{S}}_{2}$ such that:

$$
\underline{S}_{1}=\left[\begin{array}{cc}
s_{1} & 0 \\
0 & s_{1}\left[\left|\frac{g_{11} g_{12}}{g_{21} g_{22}}\right|^{\frac{1}{2}}\right.
\end{array}\right] \text { and } \quad \underline{s}_{2}=\left[\begin{array}{cc}
s_{2} & 0 \\
0 & s_{2}\left[\mid \frac{g_{11} g_{21}}{g_{12} g_{22}}\right]^{\frac{1}{2}}
\end{array}\right]
$$

where $s_{1}$ and $s_{2}$ are any non-zero real numbers. 
Proof of Theorem 16:

Consider an arbitrary nxn transfer matrix $\underline{\underline{G}}$ with non-zero entries and consider the scaling matrices $\underline{S}_{1}$ and $\stackrel{\mathrm{S}}{=}_{2}$.

Let

$$
\underline{\underline{G}}^{\prime} \quad=\quad \underline{S}_{1} \stackrel{G}{\underline{S}} \underline{S}_{2}
$$

Then

$$
\underline{\underline{G}}^{\prime} * \underline{\underline{G}}^{\prime}=\left(\underline{\underline{S}}_{1} \underline{\underline{G}} \underline{\underline{S}}_{2}\right) *\left(\underline{\underline{S}}_{1} \stackrel{\underline{G}}{\underline{S}_{2}}\right)
$$

or

$$
\underline{G}^{\prime} * \underline{G}^{\prime}=\underline{S}_{2}\left(\underline{G}^{*} \underline{S}_{1}^{2} \underline{G}\right) \underline{S}_{2}
$$

This last step is made possible by the fact that $\underline{S}_{1}$ and $\underline{\underline{S}}_{2}$ are real diagonal matrices. The minimal condition number wil1 take on a value of unity if and only if the eigenvalues of $\underline{G}^{\prime *} \underline{G}^{\prime}$ are all equal. This in turn will only be true if and only if $\underline{G}^{*} \underline{S}_{1}^{2} \underline{G}$ is a diagonal matrix. For an nxn system, this requires that $n(n-1) / 2$ offdiagonal elements with $n$ unknowns (the scaling parameters) be equal to zero. For systems of order higher than 3 (Table I) this is clearly impossible. 
Proof of Corollary 1

This corollary can be proved using arguments similar to those used in proving Theorem 1. The CE for the closed loop system of Figure 4 is again given by Equation (A1) .

A rational transfer matrix $\underline{\underline{H}}(\mathrm{~s})$ with $\operatorname{det}(\underline{\mathrm{H}}(0))<0$ will be integral controllable only if all the coefficients in det (sd(s) $\stackrel{I+k N}{=}(\mathrm{s})$ ) are negative for arbitrarily small values of $k$. If $\underset{H}{H}(\mathrm{~s})$ is improper, the coefficient of the highest power of $s$ in $\operatorname{det}(s d(s) \underset{I}{I}+\underset{N}{N}(s))$ is of the form:

$$
a_{n}=\Pi_{n}+k \cdot c
$$

Where $\pi_{n}$ is the coefficient of the highest power of $s$ in $d(s)$ and $c$ is a constant which depends on $\stackrel{N}{=}(s)$. Of course a necessary condition for $\underset{=}{H}(s)$ to be stabilizable is that $c<0$. In this case however,

$$
\operatorname{Lim}_{k \rightarrow 0} a_{n}=i_{n}>0
$$

Thus, for sufficiently small values of $k$, the coefficient of the highest power of $\mathrm{s}$ in det $(\mathrm{sd}(\mathrm{s}) \stackrel{\mathrm{I}}{=}+\mathrm{kN}(\mathrm{s}))$ becomes positive while the constant term $\operatorname{det}(\mathrm{kN}(0))$ remains negative. Such systems cannot be stable. $\quad \underline{\text { QED }}$ 
Proof of Corol1ary 2:

Condition (a) of the corollary implies det $(\stackrel{\mathrm{H}}{\underline{(}(0))}>0$. Since

$$
\lambda_{j j} \stackrel{(G)}{=} h_{j j} \frac{\operatorname{det}\left(H_{=}^{j j}(0)\right.}{\operatorname{det}(\underline{\underline{H}}(0)}
$$

condition (b), together with (a) and $\lambda_{j j}>0, j=1-3$ imply

$$
\operatorname{det}\left(\underline{\underline{H}}^{j \mathrm{j}}(0)\right)>0, j=1-3
$$

Final1y, condition (b) together with (A24) imply

$$
Z_{i}\left(\underline{H}^{j j}(0)\right) \varepsilon \underline{C}^{+}, \quad i=1,2 ; j=1-3
$$

where the $Z_{i}$ 's denote the eigenvalues of $\stackrel{H}{=}^{j j}(0)$. By virtue of Theorem 7 each of the three $2 \times 2$ subsystems $\underline{\underline{H}}^{j \mathrm{j}}(\mathrm{s})$ is integral controllable. Thus $\underline{H}(s)$ is $j-S F T / A F T, j=1-3$. 
Justification of Remark 1:

Recal1 the Left Hand Side of (A18):

$$
\frac{\|\underline{\underline{G}}-\underline{\underline{G}}\|}{\|\tilde{\underline{G}}\|} \cdot\|\underline{\underline{G}}\| \cdot\left\|\underline{\underline{G}}^{-1}\right\|
$$

Since (A25) is an upper bound for the eigenvalues of

$$
\underline{\underline{G}}^{-1}(\underline{\underline{G}}-\underline{\tilde{G}})
$$

(A18) is sufficient to guarantee that the eigenvalues of (A26) have a magnitude less than unity irrespective of how $\underline{\underline{G}}(\mathrm{~s})$ and $\underline{\underline{G}}(\mathrm{~s})$ are scaled. Scaling of $\underline{\underline{G}}(s)$ and $\underline{\underline{G}}(s)$ by diagonal matrices will leave the eigenvalues of (A26) unchanged but can make (A25) arbitrarily large. For this reason, scaling should be done with the aim of minimizing (A25).

This, however, is not a very practical approach since it requires knowledge of $\underline{\underline{G}}$. Fortunately, numerical results show that the term

$$
\frac{\|\underline{\underline{G}-\tilde{G}}\|}{\|\underline{\underline{G}}\|}
$$

in (A25) is only weakly scaling dependent. Specifically, these results show that (A27) is bounded above by the maximum relative change in the elements

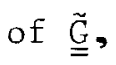

$$
\max \left|\frac{g_{i j}-\tilde{g}_{i j}}{\tilde{g}_{i j}}\right| i, j=1, n
$$

irrespective of how $\underline{\underline{G}}$ and $\tilde{\underline{G}}$ are scaled. Thus, instead of scaling such that (A25) is minimized, it is sufficient to scale such that the condition number is minimized. The simplicity introduced by this approximation offsets the fact that the resulting inequality (cf. (A18)) is not necessarily the least conservative. 
APPENDIX B

Example 13:

Consider the control structure shown in Figure 4. Let us assume $\underline{\underline{C}}(\mathrm{~s})=I$ and consider the steady state gain matrix

$$
\stackrel{\mathrm{H}}{=}_{7}=\left[\begin{array}{lll}
1.5 & 4.8 & 4.8 \\
7.9 & 8.8 & 7.4 \\
6.9 & 9.0 & 3.4
\end{array}\right]
$$

with

$$
\frac{\operatorname{det}\left(\underline{\underline{H}}_{7}\right)}{\prod_{i=1}^{3} h_{i i}}=2.5
$$

but with eigenvalues in the left half plane:

$$
\begin{aligned}
& \mathrm{Z}_{1}\left(\stackrel{\mathrm{H}}{=}_{7}\right)=18.8 \\
& \mathrm{Z}_{2}\left(\stackrel{\mathrm{H}}{=}_{7}\right)=-1.82 \\
& \mathrm{Z}_{3}\left(\stackrel{(}{=}_{7}\right)=-3.31
\end{aligned}
$$

By virtue of Theorem 7 , the system $\mathrm{H}_{7}$ is not integral controllable. By virtue of Theorem 1, it may be conditionally controllable. This will be the case if the characteristic loci corresponding to the negative eigenvalues of $\underline{\mathrm{H}}_{7}$ were to cross the negative real axis at finite values. For any constant matrix, this is impossible and therefore the closed loop system will be unstable for all values of $k>0$, i.e., the system will be "structurally monotonic unstable". 
Example 14:

This example will show that Theorem 12 breaks down if condition (b) is violated. Consider the following semi-proper transfer matrix:

$$
\underline{G}_{4}(s)=\left[\begin{array}{ll}
2 & -7 \\
2 & \frac{-5(s+1)}{(2 s+1)}
\end{array}\right]
$$

with $\lambda_{11}=-2.5$

Assume $\mathrm{y}_{2}$ is controlled through $\mathrm{u}_{2}$ with an integral controller $\mathrm{gc}_{2}(\mathrm{~s})$ and let

$$
\mathrm{gc}_{2}(\mathrm{~s})=\frac{(\mathrm{s}+1)}{\mathrm{s}}
$$

then $\quad g_{11, \mathrm{CL}}(\mathrm{s})=g_{11, \mathrm{OL}}(\mathrm{s})-\frac{\mathrm{gc}_{2}(\mathrm{~s}) \mathrm{g}_{12}(\mathrm{~s}) \mathrm{g}_{21}(\mathrm{~s})}{1+\mathrm{gc}_{2}(\mathrm{~s}) \mathrm{g}_{22}(\mathrm{~s})}$

and, upon substitution,

$$
g_{11, C L}(s)=-\frac{\left(22 s^{2}+24 s+4\right)}{3 s^{2}+9 s+5}
$$

Clearly $g_{11, C L}(s)$ has neither a RHP zero or a RHP pole in spite of the fact that $\lambda_{11}<0$. 
Example 15:

This last example will show that Theorem 12 also breaks down if condition (c) is violated. Consider the transfer matrix

$$
\underline{G}_{7}(s)=\left[\begin{array}{cc}
\frac{2}{2 s+1} & 7 \\
2 & 5
\end{array}\right]
$$

with $\lambda_{11}=-2.5$.

As in Example 15, if

$$
\mathrm{gc}_{2}(\mathrm{~s})=\frac{(\mathrm{s}+1)}{\mathrm{s}}
$$

then

$$
g_{11, C L}(s)=-\frac{\left(28 s^{2}+30 s+4\right)}{(2 s+1)(6 s+5)}
$$

which is stable and minimum phase despite $\lambda_{11}<0$. 
REFERENCES

Bode, H. "Network Analysis and Feedback Amplifier Design", D. Van Nostrand, New York, 1945.

Bristol, E. H. On a New Measure of Interaction for Multivariable Process Contro1, IEEE Trans. Autom. Contro1, 1966, AC-11, 133.

Bristol, E. H. RGA 1977: Dynamic Effects of Interaction, 16th IEEE Conference on Decision and Contro1, New Orleans, LA, 1977.

Forsythe, G. E.; Moler, C. B. "Computer Solution of Linear Algebraic Systems", Prentice-Ha11, Inc., Englewood Cliffs, NJ, 1967, pp. 20-26.

Gagnepain, J. P.; Seborg, D. E. Analysis of Process Interactions with Applications to Multiloop Control System Design, Ind. Eng. Chem. Process Des. Dev., 1982, 21, 5 .

Holt, B. R.; Morari, M. Design of Resilient Processing Plants. The Effect of Right-Ha1f-Plane Zeros on Dynamic Resilience, Chem. Eng. Sci., in press, 1984.

Jafarey, A.; McAvoy, T. J. Degeneracy of Decoupling in Distillation Columns , Ind. Eng. Chem. Process Des. Dev., 1978, 17, 485.

Johnson, L. W.; Riess, R. D. "Numerical Analysis", Addison-Wesley Publishing Co., Reading MA, 1977, pp 40-41.

MacFarlane, A. G. J. A Survey of Some Recent Results in Linear Multivariable Feedback Theory, Automatica, 1972, 8, 455.

McAvoy, T. J. "Interaction Analysis", ISA Monograph, Research Triangle Park, NC, 1983.

Morari, M. "Robust Stability of Systems with Integral Control", 22nd IEEE Conference on Decision and Control, San Antonio, Texas, 1983. 
Niederlinski, A. A Heuristic Approach to the Design of Linear Multivariable Interacting Control Systems, Automatica, 1971, 7, 691.

Ray, W. H. "Advanced Process Control", McGraw-Hill Book Company, Inc., New York, 1981, p. 69.

Rosenbrock, H. H. An Automatic Method for Finding the Greatest or Least Value of a Function, Computer J., 1960, 3, 1975.

Rosenbrock, H. H. "Computer-Aided Control System Design", Academic Press, New York, 1974.

Sande11, H.; Athans, M. On "Type L" Multivariable Linear Systems, Automatica, $1973,9,131$.

Shinskey, F. G. "Process Control Systems", McGraw-Hil1 Book Company, Inc., New York, 1967, pp. 188-189.

Shinskey, F. G. "The Stability of Interacting Control Loops With and Without Decoupling", Proc. IFAC Multivariable Technological Systems Conf., Univ. of New Brunswick, 1977, pp. 21-30.

Shinskey, F. G. "Process Control Systems", McGraw-Hill Book Company, Inc., New York, 1979, pp. 196-212. 
TABLE I

\begin{tabular}{lccc}
\hline $\begin{array}{c}\text { System } \\
\text { order }\end{array}$ & $\begin{array}{c}\text { Number of scaling } \\
\text { parameters }\end{array}$ & $\begin{array}{c}\text { Number of } \\
\text { equations }\end{array}$ & $\begin{array}{c}\text { Degrees of } \\
\text { freedom }\end{array}$ \\
\hline 2 & 2 & 1 & 1 \\
3 & 3 & 3 & 0 \\
4 & 4 & 6 & -2 \\
$\mathrm{n}$ & $\mathrm{n}$ & $\mathrm{n}(\mathrm{n}-1) / 2$ & $\mathrm{n}(\mathrm{n}-3) / 2$ \\
\hline
\end{tabular}


FIGURE CAPTIONS

Figure 1. B1ock diagram for $2 \times 2$ system.

Figure 2. Block diagram for $2 \times 2$ system with controller on loop 2.

Figure 3. $\mu_{1}^{-1}(\mathrm{~s})$ as a multiplicative perturbation on $\left(y_{1} / u_{1}\right)$ OL $=g_{11}(s)$.

Figure 4. Basic MIMO integral control configuration.

Figure 5. $\quad \gamma^{*}$ versus $\|\Lambda\|_{1}$ for $2 \times 2$ systems, analytical results

Figure 6. $\quad \gamma^{*}$ versus $\|\underline{\underline{\Lambda}}\|_{1}$ for $3 \times 3$ systems, numerical results.

Figure 7. $\gamma^{*}$ versus $\max \left[\|\underline{=}\|_{1},\|\Lambda\|_{\infty}\right]$ for $4 \times 4$ systems, numerical results. 


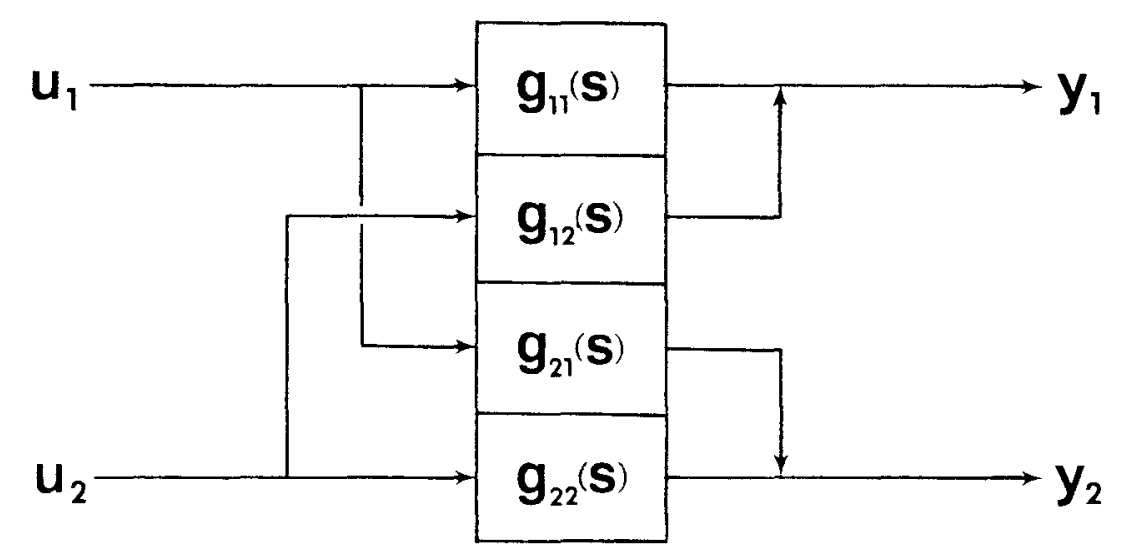

Figure 1. Block diagram for $2 \times 2$ system.

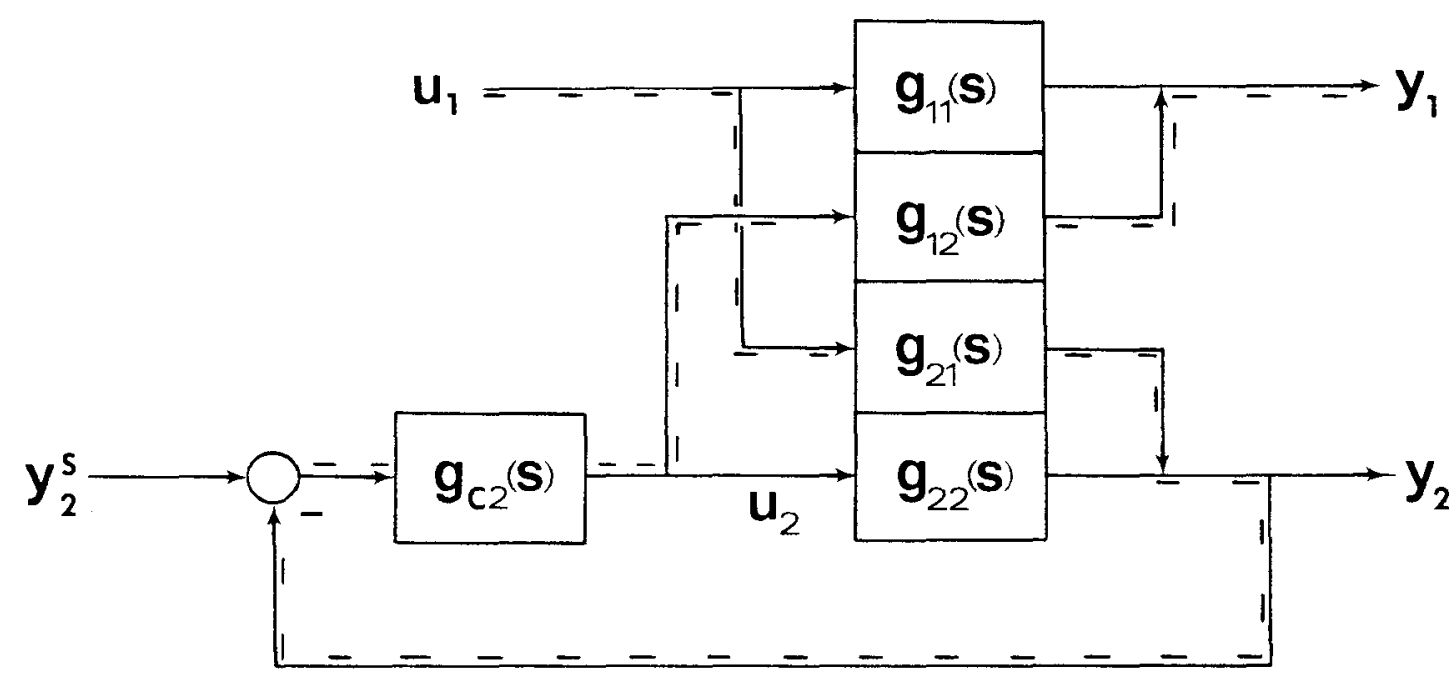

Figure 2. Block diagram for $2 \times 2$ system with controller on $100 \mathrm{p} 2$. 


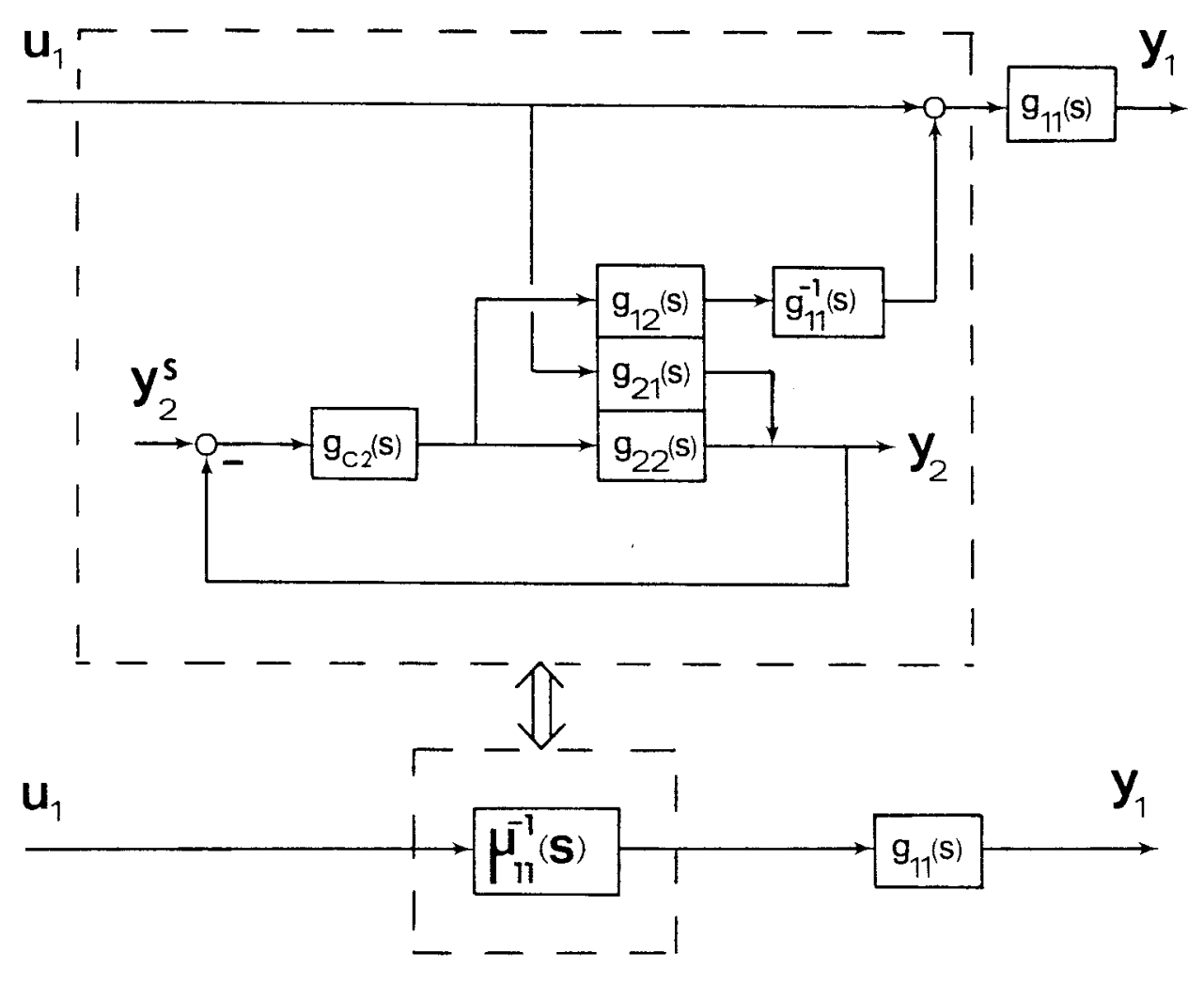

Figure 3. $\mu_{1}^{-1}(s)$ as a multiplicative perturbation on $\left(y_{1} / u_{1}\right)_{\text {OL }}=g_{11}(s)$.

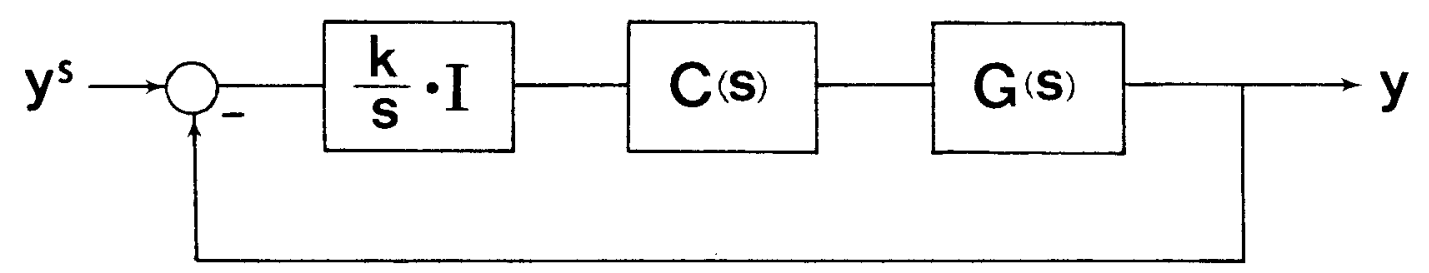

Figure 4. Basic MIMO integral control configuration. 


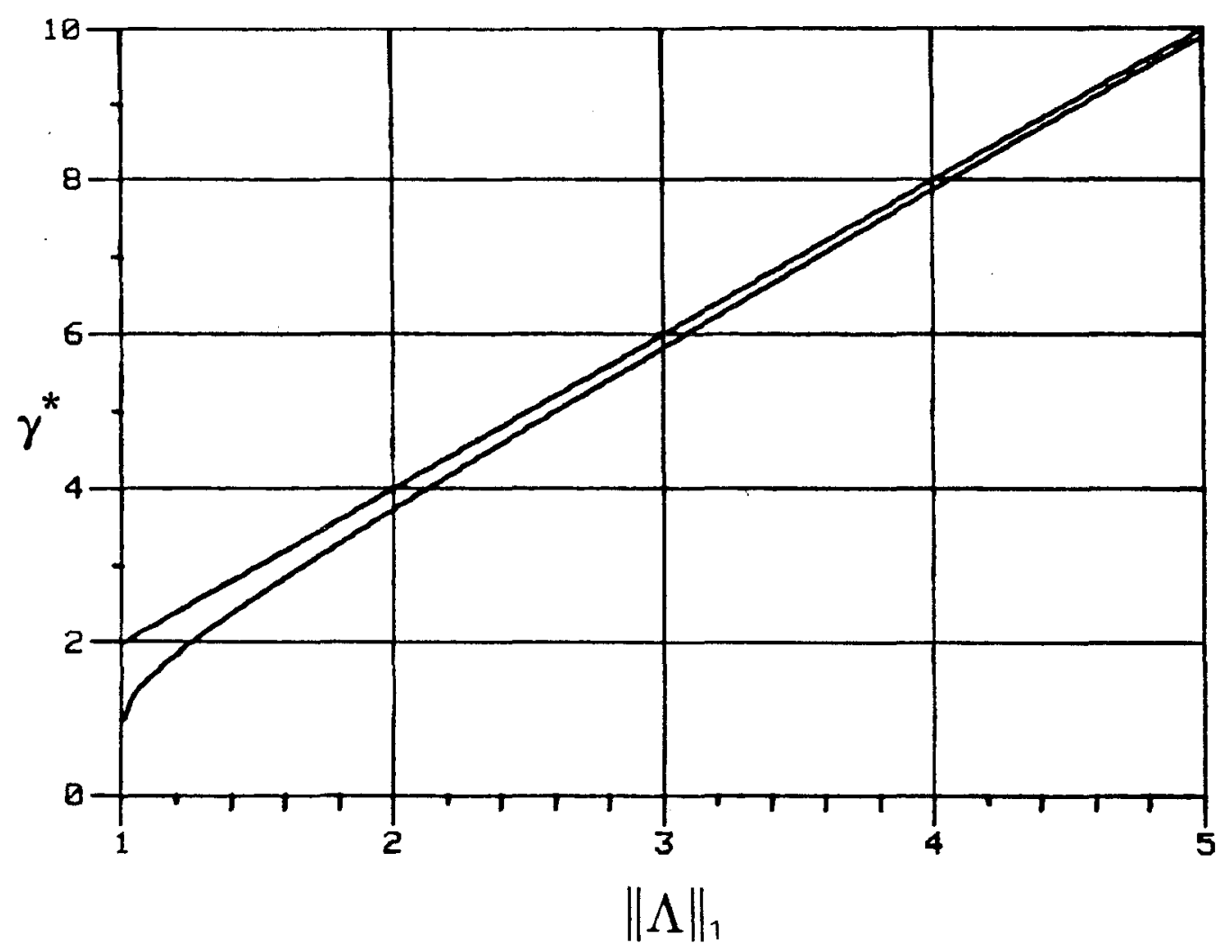

Figure 5. $\quad \gamma^{*}$ versus $\|\Lambda\|_{1}$ for $2 \times 2$ systems, analytical results. 


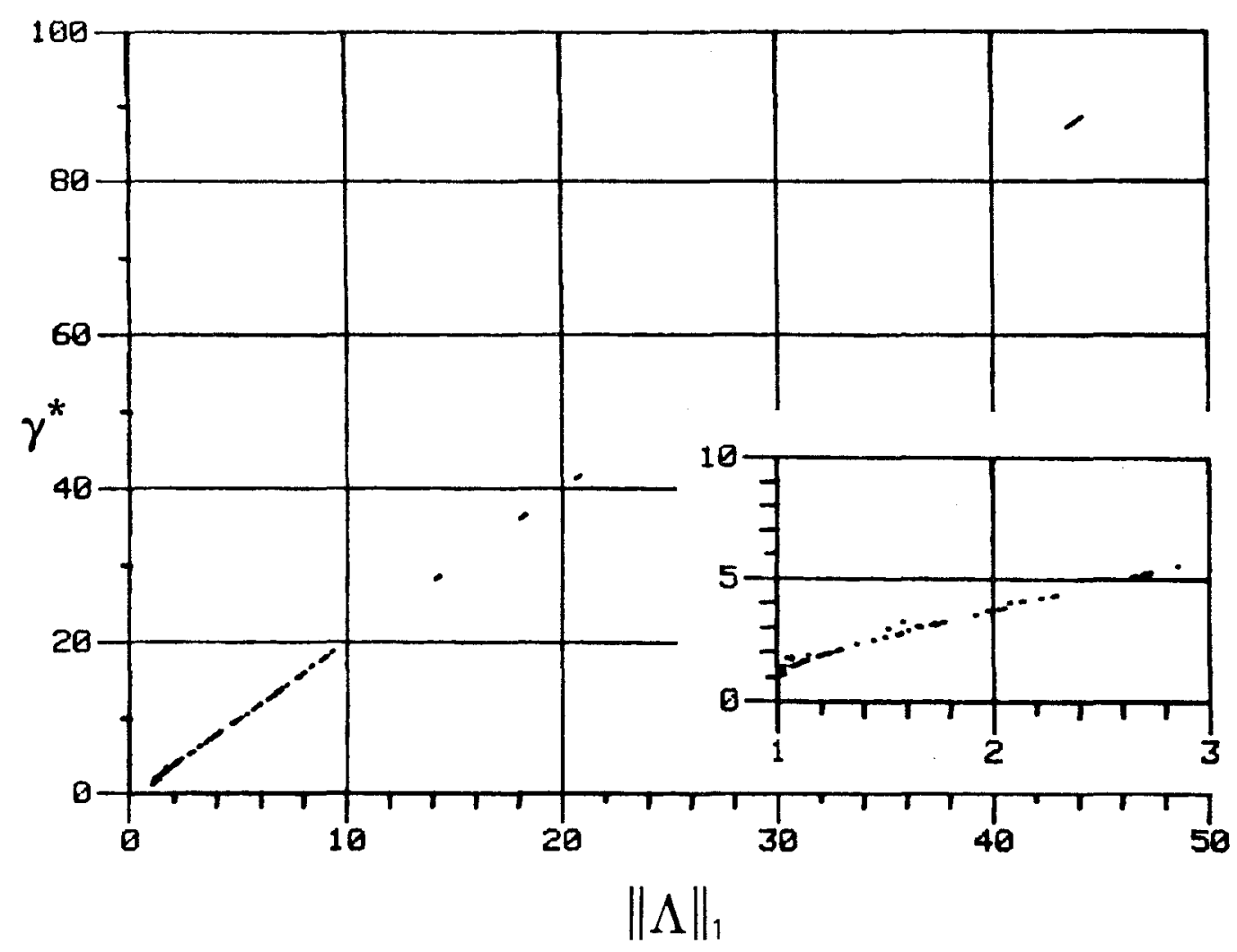

Figure 6. $\quad \gamma^{*}$ versus $\|\Lambda\|_{1}$ for $3 \times 3$ systems, numerical results. 


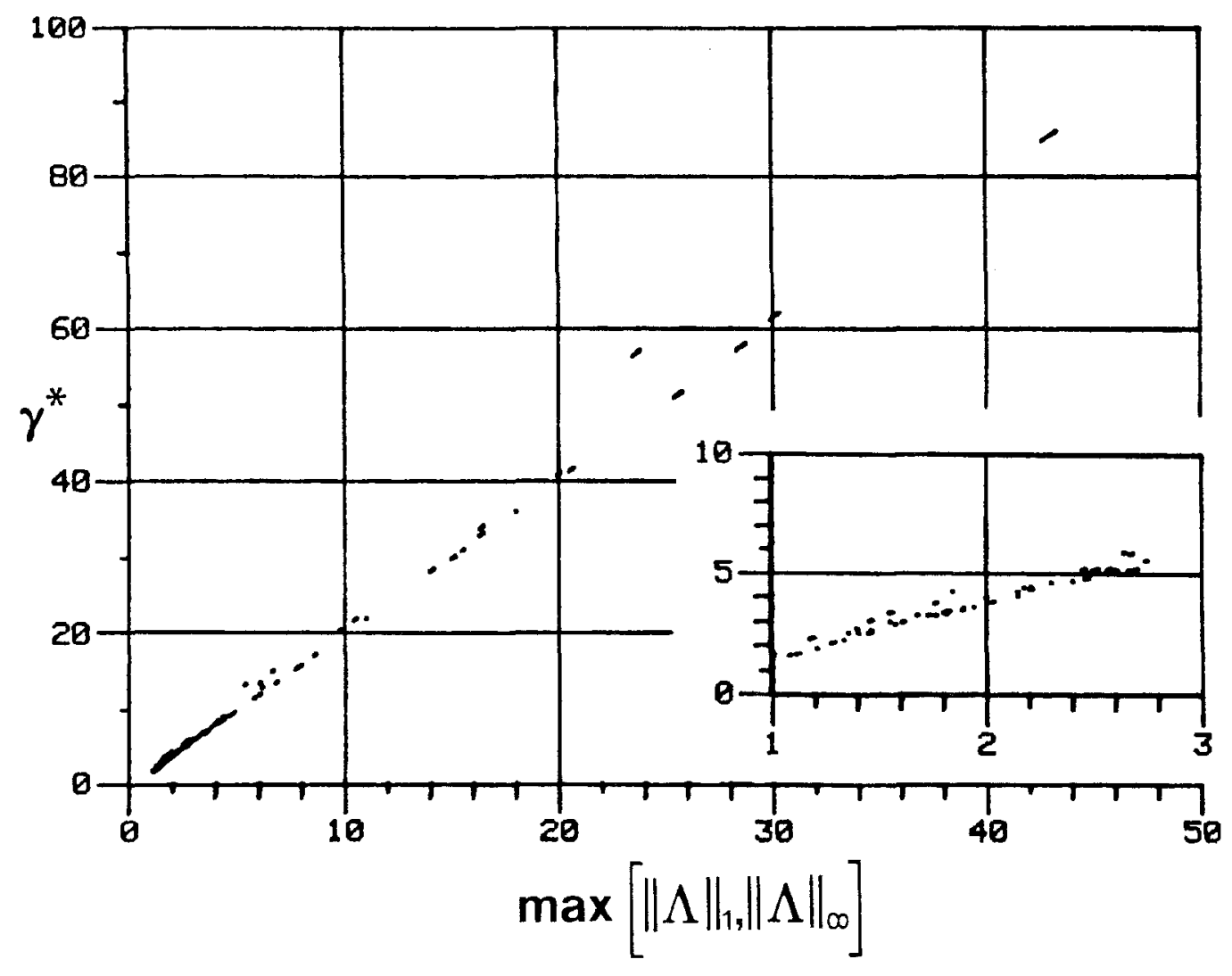

Figure 7. $\gamma^{*}$ versus $\max \left[\|\Lambda\|_{1},\|\Lambda\|_{\infty}\right]$ for $4 \times 4$ systems, numerical results. 
CHAPTER III: THE $\mu$ INTERACTION MEASURE 
INTRODUCTION

The trend in the chemical process industries is towards increased process integration. It has been followed, in turn, by a need for more advanced high-performance control systems. Practicing control engineers are now frequently faced with the task of designing controllers for multivariable plants with more than just one or two inputs and outputs. Ideally a multivariable plant would be controlled by a single multivariable controller where the control action of each manipulated variable is a function of all the measurements. In practice, in the chemical process industries, multivariable controllers are avoided in favor of simpler "decentralized" controllers. In this paper we mean by decentralized control that the controller $\mathrm{C}(\mathrm{s})$ for a plant $\mathrm{G}(\mathrm{s})$ is blockdiagonal (Fig. 1).

$$
u_{i}=c_{i}(s)\left(y_{i}-r_{i}\right)
$$

Here we have assumed that $\mathbf{u}$ and $\mathbf{y}$ are the vectors of inputs and outputs, respectively, for the $n \times n$ transfer function matrix $G(s)$. $r$ is the vector of reference signals or setpoints for the closed loop system. It is further assumed that $\mathbf{u}, \mathbf{y}$ and $\mathbf{r}$ have been partitioned in the same manner $u=\left(u_{1}, u_{2}, \ldots u_{m}\right)^{T}, y=\left(y_{1}, y_{2}, \ldots y_{m}\right)^{T}, r=\left(r_{1}, r_{2}, \ldots r_{m}\right)^{T}$ as the controller. A distillation column controlled with two or more SingleInput Single-output (SISO) control loops is an example of a multivariable plant under feedback with a diagonal or "fully-decentralized" controller.

Decentralized control structures are desirable because they offer many practical advantages. Not least among these is the fact that by carefully selecting the variable pairings, a multivariable control problem can be effectively reduced to several SISO problems. Not only does this result in fewer tuning parameters, but the tuning can be left 
to some relatively unspecialized personnel. In contrast, an advanced multivariable controller would almost invariably require the presence of a specialist.

An additional reason for using decentralized control structures lies with the fact that more complex controllers simply may not be needed. Consider the control system shown in Fig. $2 \mathrm{~A}$ for the $2 \times 2$ plant $\mathrm{G}(\mathrm{s})=$ $g_{i j}(s),(i, j=1,2)$. It is reasonable to ignore the of $f$-diagonal plant transfer functions $g_{12}(s)$ and $g_{21}(s)$ if these are "small" relative to $g_{11}(s)$ and $g_{22}(s)$ (Fig. 2B). A decentralized controller that makes the closed-loop system in Fig. $2 \mathrm{~B}$ stable should also stabilize that in Fig. $2 \mathrm{~A}$ with little or no loss of performance.

These last two remarks are important because they convey the two key ideas that will allow us to develop a mathematical treatment of decentralized control structures. The first is that an $n \times n$ plant $G(s)$ can be approximated by a block-diagonal plant $\tilde{\mathrm{G}}(\mathrm{s})$ if the off-diagonal blocks of $\mathrm{G}(\mathrm{s})$ are sufficiently "small". The second is that for sufficiently "close" plants $G(s)$ and $\tilde{G}(s)$, a block-diagonal controller $\mathbf{C}(\mathrm{s})$ can be designed to make the feedback loop around $\tilde{\mathbf{G}}(\mathrm{s})$ stable with the assurance that the feedback 100 around $G(s)$ will be stable as well.

The task of determining which blocks in $\mathrm{G}(\mathrm{s})$ can be ignored is akin to the pairing problem in fully-decentralized control systems. In both cases we seek to identify the "dominant" transfer functions in $G(s)$. A $2 \times 2$ system, for example, offers two alternatives: $G(s)$ can be approximated by

$$
\tilde{G}_{1}(s)=\left[\begin{array}{cc}
g_{11}(s) & 0 \\
0 & g_{22}(s)
\end{array}\right] \text { or } \quad \tilde{G}_{2}(s)=\left[\begin{array}{cc}
0 & g_{12}(s) \\
g_{21}(s) & 0
\end{array}\right]
$$

and therefore a decentralized controller can be implemented on the 
diagonal or of $f$-diagonal transfer functions in $\mathbf{G}(\mathrm{s})$. A $3 \times 3$ system, on the other hand, offers 15 alternatives. Clearly the number of alternatives grows rapidly for systems with more than two inputs and two outputs, and the selection of a control structure becomes a problem of significant importance. The purpose of an Interaction Measure would be to assist the control engineer in this selection by quantifying the difference between a plant $\mathbf{G}(\mathrm{s})$ and its approximation $\tilde{\mathbf{G}}(\mathrm{s})$.

Such a measure should also guide the engineer in the tuning of the block-diagonal controller $\mathbf{C}(s)$ for the plant $\mathbf{G}(\mathrm{s})$. Even if the difference between $\mathrm{G}(\mathrm{s})$ and $\tilde{\mathrm{G}}(\mathrm{s})$ is small, selecting $\mathrm{C}(\mathrm{s})$ such that the feedback loop around $\tilde{\mathrm{G}}(\mathrm{s})$ is stable does not guarantee the stability of the feedback loop around the real plant $G(s)$. Ignoring the off-diagonal system blocks results in interactions which can lead to performance deterioration and even instability.

For our further developments we will make a more precise definition of "Interaction Measure" with reference to Fig. 3:

A controller

$$
C(s)=\operatorname{diag}\left(C_{1}(s), C_{2}(s), \ldots C_{m}(s)\right)
$$

is to be designed for the system

$$
\tilde{\mathbf{G}}(\mathrm{s})=\operatorname{diag}\left(\mathbf{G}_{11}(s), \mathbf{G}_{22}(s), \ldots \mathbf{G}_{m m}(s)\right)
$$

such that the block diagonal closed loop system with the transfer matrix

$$
\tilde{\mathrm{H}}(\mathrm{s})=\tilde{\mathrm{G}}(\mathrm{s}) \mathrm{C}(\mathrm{s})(\mathrm{I}+\tilde{\mathrm{G}}(\mathrm{s}) \mathrm{C}(\mathrm{s}))^{-1}
$$

is stable ( $\delta=0$ in Fig. 3). An IM expresses the constraints imposed on the choice of the closed loop transfer matrix $\tilde{\mathrm{H}}(\mathrm{s})$ for the block diagonal system which guarantee that the full closed loop system

$$
H(s)=G(s) C(s)(I+G(s) C(s))^{-1}
$$

is stable (i.e., $\delta=1$ in Fig. 3). 
The only justification for most proposed dynamic IM's (Davison, 1970; Witcher \& McAvoy, 1977; Tung \& Edgar, 1981; Gagnepain \& Seborg, 1982) is that they become unity or zero for diagonal or triangular systems and tend to increase as the off-diagonal elements increase in magnitude. Though this is certainly an essential property of any IM, it does not by itself define a scale according to which the severity of the interactions is to be judged. Generally the implicitly employed scale does not have a theoretical justification but is only "proven" through a couple of case studies. In this case one might as well look at the system transfer matrix directly and not confuse the issue by using an arbitrary IM which again has to be judged subjectively. The recent correspondence between Jensen (1985) and McAvoy (1985) is a good example of this confusion which we hope to clear up through this work.

Nevertheless our definition of an IM has its limitations and therefore the results should be interpreted with caution. Though the definition of the IM guarantees $\mathbf{H}(\mathbf{s})$ to be stable it can be very badly behaved. The IM might indicate "small" interactions but the performance could be arbitrarily poor. This will be demonstrated in one of the examples in this paper. The problem of achieving a selected performance objective with a decentralized controller is the subject of current research.

The following developments will show that the matrix $(G(s)-\tilde{G}(s)) \tilde{G}^{-1}(s)$ plays a central role in interaction analysis. For simplicity of notation, we define the matrix

$$
E(s)=(G(s)-\tilde{G}(s)) \tilde{G}^{-1}(s)
$$

E(s) can be viewed as the "relative error" arising from the "approximation" of the full system $G(s)$ by the block diagonal system 
$\tilde{\mathrm{G}}(\mathrm{s})$.

STABILITY CONDITIONS FOR DECENTRALIZED CONTROL SYSTEMS

The development of an IM requires a precise understanding of the conditions under which a decentralized controller will make the two closed-loop transfer matrices (5) and (6) stable. Let us denote by $\mathrm{N}(\mathrm{k}$, $g(s))$ the net number of clockwise encirclements of the point $(k, 0)$ by the image of the Nyquist $D$ contour under $g(s)$. An application of the multivariable Nyquist criterion to the control system in Fig. 1 yields the following stability conditions.

Theorem 1: Assume that $\mathrm{G}(\mathrm{s})$ and $\tilde{\mathrm{H}}(\mathrm{s})$ are stable. Then the closed loop system $H(s)$ is stable if and only if

$$
\mathrm{N}(\mathrm{O}, \operatorname{det}(\mathrm{I}+\mathrm{E}(\mathrm{s}) \tilde{\mathrm{H}}(\mathrm{s})))=0
$$

Proof: The proof of all the theorems in this paper will be found in an earlier publication (Grosdidier and Morari, 1985).

Theorem 1 can be extended to open-1oop unstable systems with a rather restrictive set of assumptions. This situation has been investigated by Grosdidier \& Morari (1985). An exception are systems with integrators: Woolverton (1980) uses the model of a liquid-liquid separator which has one unstable pole at the origin. The diagonal system $\tilde{G}(\mathrm{~s})$ has the same unstable pole. In this case, if $\tilde{\mathbf{H}}(\mathrm{s})$ is stable then (8) is both a necessary and sufficient condition for the stability of $\mathrm{H}(\mathrm{s})$.

Inspection of (8) points to the importance of the matrix $E(s)=$ $(G(S)-\widetilde{G}(s)) \widetilde{G}^{-1}(s)$ in the assessment of interactions. Ideally one would want to select $\tilde{\mathrm{H}}(\mathrm{s})=\mathrm{I}$, i.e., perfect control. If $\mathrm{G}(\mathrm{s})=\tilde{\mathrm{G}}(\mathrm{s})$, i.e., $\mathrm{G}(\mathrm{s})$ itself is block diagonal, then $\mathbf{E}(\mathrm{s})=0$ and ( 8 ) is trivially satisfied. 
Thus the closed loop system $\mathrm{H}(\mathrm{S})$ is stable regardless of how $\tilde{\mathrm{H}}(\mathrm{s})$ is chosen. If $\mathrm{G}(\mathrm{s}) \neq \tilde{\mathrm{G}}(\mathrm{s}), \tilde{\mathrm{H}}(\mathrm{s})$ has to be chosen such that (8) remains satisfied. Qualitatively, at least, it is clear that when $\mathrm{E}(\mathrm{s})$ is "large" $\tilde{\mathbf{H}}(\mathrm{s})$ has to be made "small" to avoid encirclements. A small $\tilde{\mathbf{H}}(\mathrm{s})$ implies poor performance. Thus the control structure should be selected such that $\mathbf{E}(\mathrm{s})$ is "small" and one can select $\tilde{\mathbf{H}}(\mathrm{S})=\mathbf{I}$, at least for some frequency range, and still satisfy (8). The development of an IM in the next section is based on this observation.

As an illustration of the importance of properly selecting the control structure, consider the following corollary.

Corollary 1.1: Assume that

i) $\quad \mathrm{G}(\mathrm{s})$ and $\tilde{\mathrm{H}}(\mathrm{s})$ are strictly stable

ii) $\quad G(s) C(s)$ is strictly proper

iii) $\tilde{\mathrm{H}}(\mathrm{S})$ has vanishing tracking error for asymptotically constant inputs, i.e., $\tilde{\mathrm{H}}(0)=\mathrm{I}$.

then the closed loop system $\mathbf{H}(\mathrm{s})$ will be unstable if

$$
\operatorname{det}\left(\mathbf{G}(0) \tilde{G}^{-1}(0)\right)<0
$$

If the gain matrices $\mathrm{G}(0)$ and $\tilde{\mathrm{G}}(0)$ are sufficiently different (9) might be satisfied. Then there exist no decentralized controller that can make (5) and (6) stable.

Note that Corollary 1.1 is a generalization of Niederlinski's theorem and condition (Grosdidier et al., 1985)

$$
\frac{\operatorname{det}(G(0))}{n}<0
$$

to block diagonal controllers. Its main limitation is that it is not 
sufficient for stability. Even if $\operatorname{det}\left(\mathrm{G}(0) \tilde{\mathrm{G}}^{-1}(0)\right)>0$, there may not exist a controller such that (8) is satisfied, except in the case of $2 \times 2$ systems.

THE $\mu$ INTERACTION MEASURE

Although Theorem 1 is useful to single out the importance of the "error" matrix E(s), it offers, as such, no apparent benefit over the multivariable Nyquist criterion. Its real usefulness comes from our ability to derive sufficient stability conditions for $\mathrm{H}(\mathrm{s})$ in the form of bounds on $\tilde{\mathbf{H}}(\mathrm{s})$. This next theorem provides a sufficient condition for (8).

Theorem 2: Assume that $\mathrm{G}(\mathrm{s})$ and $\tilde{\mathrm{H}}(\mathrm{s})$ are stable. Then the closed loop system $\mathrm{H}(\mathrm{s})$ is stable if

$$
|| \tilde{H}(j \omega)||<|| E(j \omega)||^{-1} \quad \forall \omega
$$

where || $\mathbf{A}||$ denotes any induced norm of $\mathbf{A}$.

According to our definition ||$E(j \omega)||^{-1}$ is an example of an IM. (11) is the condition under which a controller that makes $\tilde{\mathrm{H}}(\mathrm{s})$ stable will also make $\mathrm{H}(\mathrm{s})$ stable. It states that for stability the magnitude of the diagonal blocks $\tilde{\mathbf{H}}(j \omega)$ has to be constrained by the reciprocal of the norm of the error matrix $\mathrm{E}(\mathrm{j} \omega)$. The control structure should therefore be selected such that || $\mathbf{E}(j \omega)||$ is small for as large a frequency range as possible.

||$E(j \omega)||^{-1}$ can be conveniently displayed on an amplitude-frequency diagram and serve as an upper bound for $\left\|\tilde{H}_{i}(j \omega)\right\|, \quad(i=1, n)$. Thus this IM lends itself well to graphical implementation.

||$E(j \omega)||^{-1}$ is an intuitively appealing IM. Its disadvantage lies in its conservatism (11) makes no assumptions about the structure of 
$\tilde{\mathbf{H}}(\mathrm{s})$. $\tilde{\mathrm{H}}(\mathrm{S})$ could actually be a full matrix, which is not meaningful in the present context since $\tilde{\mathrm{H}}(\mathrm{s}$ ) is block-diagonal (cf. (5)). It is therefore natural to look for an expression like (11) which takes into account the block-diagonal structure of $\tilde{\mathbf{H}}(\mathrm{s})$.

The importance of taking the block-diagonal structure of $\tilde{\mathrm{H}}(\mathrm{s})$ into account is best conveyed when we investigate the effect scaling has on (11). The expression

$$
\operatorname{det}[\mathrm{I}+\mathrm{E}(\mathrm{s}) \tilde{\mathrm{H}}(\mathrm{s})]
$$

in (8) can be scaled with a similarity transformation D without affecting the stability criterion. (12) becomes

$$
\operatorname{det}\left[\mathrm{I}+\mathrm{DE}(\mathrm{s}) \tilde{\mathrm{H}}(\mathrm{S}) \mathrm{D}^{-1}\right]
$$

If we now restrict $D$ to $\mathscr{D}$, the set of all real diagonal matrices with the same block-diagonal structure as $\tilde{\mathrm{H}}(\mathrm{s})$

$$
\mathscr{D}=\operatorname{diag}\left(d_{1} I_{1}, d_{2} I_{2}, \ldots d_{m} I_{m}\right), d_{i} \varepsilon \mathbb{R}_{+},
$$

(13) can be expressed as

$$
\operatorname{det}\left[\mathrm{I}+\mathrm{DE}(\mathrm{s}) \mathrm{D}^{-1} \tilde{\mathrm{H}}(\mathrm{s})\right]
$$

A sufficient condition on $\tilde{\mathbf{H}}(\mathrm{S})$ analogous to (11) could then be simply

$$
|| \tilde{\mathrm{H}}(j \omega)||<|| \mathrm{DE}(j \omega) \mathrm{D}^{-1}||^{-1}
$$

However, since (16) is a sufficient condition for (8) irrespective of $D \varepsilon$ $\mathscr{D}$, D should be selected to make (16) the least conservative. This leads us to the following result. Theorem 3: Assume $\mathrm{G}(\mathrm{s})$ and $\tilde{\mathbf{H}}(\mathrm{s})$ stable. Then $\mathrm{H}(\mathrm{s})$ is stable if

$$
|| \tilde{\mathbf{H}}(j \omega)||<\left[\inf _{\mathbf{D} \varepsilon \mathscr{D}}|| D E(j \omega) D^{-1}||\right]^{-1} \quad \forall \omega
$$

The optimization problem defined in (17) is easily solved via the Perron-Frobenius Theorem in the case of the 1- and the $\infty$-norm (Grosdidier \& Morari, 1985). The optimization involving the Euclidean 
norm (maximum singular value norm) will be discussed shortly.

The use of scaling matrices is only one method of taking into account the structure of $\tilde{\mathrm{H}}(\mathrm{s})$. An even less conservative bound can be derived if we assume that the diagonal blocks of $\tilde{\mathbf{H}}(\mathrm{s})$ are norm-bounded in the sense of the Euclidean norm

$$
|| \tilde{H}_{i}(j \omega)|| E=\sigma_{\max }\left(\tilde{H}_{i}(j \omega)\right)<\delta(\omega) \quad \forall i, \omega
$$

or equivalently

$$
\left.|| \tilde{H}(j \omega)\right|_{E}=\sigma_{\max }(\tilde{H}(j \omega))<\delta(\omega) \quad \forall \omega
$$

where $\sigma_{\max }(\mathbf{A})$ is the maximum singular value of $\mathbf{A}$. A real positive function $\mu(E(j \omega))$ can be defined with the property that (8) is satisfied for all matrices $\tilde{H}(j \omega)$ satisfying (18) if and only if

$$
\sigma_{\max }(\tilde{\mathrm{H}}(j \omega))<\mu^{-1}(E(j \omega))
$$

Here, $\mu$ is the Structured Singular Value (SSV) defined by Doyle (1982).

Theorem 4 ( $\mu$ Interaction Measure): Assume that $\mathrm{G}(\mathrm{s}$ ) and $\tilde{\mathbf{H}}(\mathrm{s}$ ) are stable. Then the closed loop system $\mathrm{H}(\mathrm{s})$ is stable if

$$
\sigma_{\max }(\tilde{\mathrm{H}}(j \omega))<\mu^{-1}(E(j \omega)) \quad \forall \omega
$$

$\mu^{-1}(E(j \omega))$ is the "optimal" IM because (20) constitutes the tightest

possible norm bound: If there is a system $\tilde{\mathbf{H}}_{1}(\mathrm{~s})$ which violates (20)

$$
\sigma_{\max }\left(\tilde{\mathbf{H}}_{1}(j \omega)\right)>\mu^{-1}(E(j \omega))
$$

then there exists another system $\tilde{\mathbf{H}}_{2}(\mathrm{~s})$ such that

$$
\sigma_{\max }\left(\tilde{\mathrm{H}}_{1}(j \omega)\right)=\sigma_{\max }\left(\tilde{\mathrm{H}}_{2}(j \omega)\right)
$$

for which (8) is violated and $H(s)$ is unstable. Nevertheless $\mu^{-1}(E(j \omega))$ is conservative because it places only a magnitude bound on $\mathbb{H}(j \omega)$. This may be overly conservative at low frequency where $\tilde{\mathrm{H}}(j \omega)$ takes on a directionally structured form. If the controller contains integral action, for example, then 


$$
\lim _{\omega \rightarrow 0} \tilde{H}(j \omega)=I
$$

and there is conservatism in considering all norm bounded $\tilde{\mathbf{H}}(0)$ as in (18). A less conservative IM could be derived if we considered an expression like (20) which accounted for the "directionality" in $\tilde{\mathrm{H}}(\mathrm{s})$. In general, the more assumptions we make, the less conservative the resulting IM but the more demanding its computation.

As defined by Doyle (1982) the value of $\mu(E(j \omega))$ depends only on the block-diagonal structure of $\tilde{\mathrm{H}}(\mathrm{s})$. Its computation is an active area of research at present. An optimization is suggested by the upper bound derived by Doyle (1982).

$$
\mu(\mathrm{E}(j \omega)) \leq \inf _{\mathrm{D} \in \mathscr{g})} \sigma_{\max }\left(\mathrm{DE}(j \omega) \mathrm{D}^{-1}\right)
$$

where $\mathscr{D}$ is defined as in (14). The optimization implied by (24) is convex but the infimum is only equal to $\mu$ for $m \leq 3$. Thus for systems with two or three blocks $\mu(\mathrm{E}(j \omega))$ is equal to the IM introduced in Theorem 3 when the Euclidean norm is used. Unfortunately, efficient software for its computation is presently not accessible.

Experience has shown that an excellent upper bound for $\mu(E(j \omega))$ is obtained by scaling $\mathrm{E}(\mathrm{j} \omega)$ to minimize its Frobenius norm and subsequently computing its maximum singular value

$$
\mu(\mathrm{E}(j \omega)) \leq \sigma_{\max }\left(\mathrm{DE}(j \omega) \mathbf{D}^{-1}\right)
$$

where $\mathrm{D} \varepsilon \mathscr{D}$ solves

$$
\inf || D E(j \omega) D^{-1}|| F
$$

and where ||$A|| F=\sqrt{\sum_{i j}\left|a_{i j}\right|^{2}}$ is the Frobenius norm of A. A simple and efficient algorithm for solving (26) was published by Osborne (1960). 
It can also be shown that $\mu(E(j \omega))$ is bounded above by the PerronFrobenius eigenvalue of the matrix $|E(j \omega)|$,

$$
\mu(E(j \omega)) \leq \rho(|E(j \omega)|)
$$

where $|\mathbf{A}|$ denotes the matrix $\mathbf{A}$ with all its elements replaced by their magnitude and $\rho(\mathbf{A})$ is the spectral radius of $\mathbf{A}$. The quantity $\rho(|E(j \omega)|)$ appears explicitly in the definition of generalized diagonal dominance (Mees, 1981; Limebeer, 1982). Implications of (27) on generalized diagonal dominance and on Rosenbrock's Nyquist Array (Rosenbrock, 1974) have been discussed by Grosdidier \& Morari (1985).

Note that $\mu$ treats both diagonal and block diagonal $\tilde{\mathrm{H}}(\mathrm{s})$ in a unified "optimal" manner. This offers the advantage that independent of the number of system inputs and outputs the design engineer can determine from a single curve if the selected control structure leads to significant performance deterioration or not. In particular, only if $\mu(\mathrm{E}(0))<1$ are we assured on the basis of Theorem 4 that the decentralized controller can incorporate integral action. Every effort should therefore be made to select a control structure that satisfies this condition. The implementation of a decoupler, steady-state or dynamic, to ensure $\mu(E(0))<1$ defeats the purpose of using a decentralized controller. At present the only way of selecting the least interactive control structure is by successively trying all possible alternatives.

The limitation associated with the result of Theorem 4 is that $\mu$ gives equal preference to all the loops. In some cases this may impose an early and perhaps unnecessary roll-off for some of the $\tilde{H}_{i}(s)$. The introduction of a weighting matrix $\mathbf{W}(s)$, with block-diagonal structure equal to that of $\tilde{\mathbf{H}}(\mathrm{s})$, into the expression 


$$
(\mathrm{I}+\mathrm{E}(\mathrm{s}) \tilde{\mathrm{H}}(\mathrm{s}))
$$

circumvents this problem. (28) becomes

$$
\left(\mathrm{I}+\mathrm{E}(\mathrm{s}) \mathrm{W}(\mathrm{s}) \mathrm{W}^{-1}(\mathrm{~s}) \tilde{\mathrm{H}}(\mathrm{s})\right)
$$

and the sufficient stability condition of Theorem 4 (cf. (20)) becomes

$$
\sigma_{\max }\left(\boldsymbol{W}^{-1}(j \omega) \tilde{\mathbf{H}}(j \omega)\right)<\mu^{-1}(E(j \omega) \boldsymbol{W}(j \omega)) \quad \forall \omega
$$

In practice, a proper weighting matrix will express a performance constraint imposed on one or more of the $\tilde{\mathrm{H}}_{\dot{1}}(\mathrm{~s})$ which is due, for example, to constraints in manipulated variables or plant uncertainty. The benefit of (30) is that a weighting matrix can be used to reduce the conservatism of the $\mu$ constraint (cf. (20)) on some of the $\tilde{H}_{i}(s)$ by increasing that on others. This procedure will later be illustrated in an example.

\section{IMPLICATIONS FOR 2X2 SYSTEMS}

The interaction measures for $2 \times 2$ systems which are most widely used industrially were proposed by Rijnsdorp (1965) and Bristol (1966). The Rijnsdorp Interaction Measure is defined as

$$
k(s)=\frac{g_{12}(s) g_{21}(s)}{g_{11}(s) g_{22}(s)}
$$

and the Relative Gain as

$$
\lambda=\frac{1}{1-k(0)}
$$

Theorem 1 can be rephrased specifically for $2 \times 2$ systems.

Corollary 1.2: Assume that $\mathrm{G}(\mathrm{s})$ and $\tilde{\mathrm{H}}(\mathrm{s})$ are stable. Then the closed loop system $H(s)$ is stable if and only if

$$
\mathrm{N}(+1, \mathrm{z}(\mathrm{s}))=0
$$

where

$$
z(s)=\tilde{h}_{1}(s) \tilde{h}_{2}(s) k(s)
$$


Corollary 1.2 shows that $k(s)$ is rigorously related to closed loop stability and that both the gain and the phase information of $\kappa(s)$ can be utilized to establish the constraints imposed on $\tilde{h}_{i}(s),(i=1,2)$, by the interactions. The application of Cor. 1.2 will be discussed later in the paper.

From (24) it can be easily shown that for $2 \times 2$ systems

$$
\mu(E(j \omega))=\sqrt{|k(j \omega)|}
$$

Corollary 4.1: Assume that $\mathrm{G}(\mathrm{s})$ and $\tilde{\mathrm{H}}(\mathrm{s})$ are stable. Then the closed loop system $\mathrm{H}(\mathrm{s})$ is stable if

$$
\left|\tilde{h}_{i}(j \omega)\right|<|k(j \omega)|^{-1 / 2}=\mu^{-1}(E(j \omega)) \quad \forall i, \omega
$$

Corollary 4.1 is more conservative than Cor. 1.2 because the phase information is discarded but it is much easier to apply. Corollary 4.1 establishes the Rijnsdorp Interaction Measure as optimal for 2x2 systems in the sense of the SSV $\mu$. From these results it is self evident that $\mu^{2}(E(j \omega))$ is the most natural extension of the Rijnsdorp Interaction Measure $k(s)$ to systems larger than $2 \times 2$ and to block diagonal controller structures. Other physically motivated extensions which have been proposed (Jensen et al., 1985) have no apparent theoretical justification. McAvoy (1981) has argued that a frequency dependent definition (32) of the Relative Gain $\lambda$

$$
\lambda(s)=\frac{1}{1-k(s)}
$$

could be used in a semiquantitative manner for loop tuning. If $|\kappa(j \omega)|>$ 1 then (37) satisfies

$$
|\lambda(j \omega)|<\frac{1}{|\kappa(j \omega)|-1}
$$

and for $|\kappa(j \omega)| \gg 1$ we have the approximation

$$
|\lambda(j \omega)|<|k(j \omega)|-1 / 2
$$


Thus $|\lambda(j \omega)|$ could be used instead of $|k(j \omega)|^{-1 / 2}$ in Cor. 4.1. However, this approximation holds only for $|\kappa(j \omega)| \gg 1,(|\lambda(j \omega)| \ll 1)$, i.e., when the interactions are strong and is unnecessarily conservative. Therefore the "dynamic extension" of the Relative Gain is not recommended as an Interaction Measure.

Shinskey (1979) suggests a heuristic controller tuning rule based on the Relative Gain for a special class of decentralized $2 \times 2$ systems. This class consists of systems with constant $k(s)$ and with one 10op, say 10op 2, much faster than the other. By this it is meant that the closed-10op transfer function $\tilde{h}_{1}(\mathrm{~s})$ rolls off at a frequency much lower than that of $\tilde{h}_{2}(s)$. In the chemical industries this situation occurs, for example, when $y_{1}$ is composition and $y_{2}$ is flow.

A qualitative analysis of these systems shows that closing the slow loop (10op 1) has relatively little effect on the open-loop transfer function between $u_{2}$ and $y_{2}$. Alternatively, the open-1oop transfer function between $u_{1}$ and $y_{1}$ sees its gain scaled by a factor $1 / \lambda$ at the cross-over frequency when loop 2 is closed. As a result of this, Shinskey suggests tuning each controller with the other one in manual. The controller in the slow $100 \mathrm{p}$ is then detuned by a factor $\lambda$ prior to closing both loops simultaneously. Corollary 1.2 can be used to provide theoretical justification for this tuning rule. Corollary 1.3: Assume that $\mathbf{G}(\mathrm{s})$ and $\tilde{\mathrm{H}}(\mathrm{s})$ are stable and that the $2 \mathrm{x} 2$ system satisfies the following conditions:

i) $\quad k(s)=k=$ constant

ii) loop 2 is much faster than loop 1

iii) The Nyquist plot of $g_{1 ;} c_{1}(j \omega)$ does not intersect the real axis to the left of the point $(-1,0)$, i.e., a conditionally stable single 
loop control system is not allowed.

Then the system $\mathrm{H}(\mathrm{s})$ is stable if

Case $\mathrm{A}: \quad \lambda>1$, or if

Case B: $0<\lambda<1$ and if $\mathrm{C}_{1}(\mathrm{~s})$ is detuned by a factor $\lambda$ prior to closing both loops simultaneously.

Proof: See the Appendix.

\section{EXAMPLES}

Example 1: This first example will show how different control structures affect the value of the constraint $\mu^{-1}(E(j \omega))$. We consider the distillation column of Doukas \& Luyben (1978). The transfer function matrix for this $4 \times 4$ system is shown in Table 1 . Controlled and manipulated variables are listed in Table 2. The system was scaled to make the transfer function matrix and all the variables dimensionless. Doukas \& Luyben's scaling was such that a unit change in a dimensionless variable corresponds to a $10 \%$ change in a controlled variable or to the saturation of a manipulated variable.

The authors used a fully-decentralized controller based on the output-input pairs $((1,3),(2,2),(3,4),(4,1))$. Line 1 in Fig. 4 is a plot of $\mu^{-1}(E(j \omega))$ corresponding to these pairings. It shows that a controller with integral action cannot be designed on basis of Theorem 4 since $\mu^{-1}(E(0))<1$. This constraint can be relaxed by using an alternative controller structure. Line 2 in the same figure is a plot of $\mu^{-1}(E(j \omega))$ corresponding to a fully-decentralized controller with (diagonal) pairings $((1,1),(2,2),(3,3),(4,4))$. In this case a controller with integral action is possible since $\mu^{-1}(\mathrm{E}(0))>1$. However the interactions 
limit the achievable closed-1oop bandwidth to about $0.1 \mathrm{rad} / \mathrm{min}$.

These pairings, the only ones to yield a value of $\mu^{-1}(\mathbf{E}(0))$ greater than unity, can be compared with those derived from the Relative Gain Array of the system (Table 3). Relative Gain analysis recommends that variables be paired when their Relative Gain is close to unity (Bristol, 1966; Grosdidier et al.,1985). Table 3 shows that this rule leads to variable pairings identical to those based on Theorem 4. This suggests that the RGA might be helpful in predicting the correct pairings to use in Theorem 4 - thereby avoiding many lengthy computations of $\mu$. We also note from Table 3 that the pairings chosen by Doukas and Luyben are associated with a negative Relative Gain $\left(\lambda_{41}\right)$, an indication of a system with low integrity (Morari, 1985). In fact, it can easily be verified that Doukas and Luyben's closed-10op system will become unstable should the controller in loop 1, 2, or 4 be placed in "manual" and this so irrespective of how the controllers are tuned. This integrity problem is satisfactorily solved by pairing the variables as recommended on basis of Theorem 4 .

Block-decentralized control structures can yield values of $\mu$ less conservative than fully-decentralized structures, although this is not always the case. For the system in Table 1, consider blockdecentralized structures based on the pairings $((1-2,1-2),(3,3),(4,4))$ and $((1,1),(2,2),(3-4,3-4))$. Lines 1 and 2 in Fig. 5 show plots of $\mu^{-1}(E(j \omega))$ for these two control structures, respectively. Line 3 is a plot of $\mu^{-1}(E(j \omega))$ for the diagonal control structure and is reproduced for convenience. These curves show that the constraint is slightly improved with the first block-decentralized structure but deteriorates significantly with the second. 
Example 2: In this next example, the IMC-PID controller tuning rules (Rivera et al., 1985) are used in conjunction with the $\mu$ Interaction Measure to design a diagonal, fully-decentralized controller for the system in Table 1. The Internal Model Control (IMC) design procedure applies to SISO systems and leads to PD controllers, occasionally augmented with a first order lag. These controllers have as their only tuning parameter the closed-loop time constant $\varepsilon$.

The controllers in loops 1, 2 and 3 are selected by applying the IMC tuning rules to second order lag approximations of $g_{11}(s), g_{22}(s)$ and $\mathrm{g}_{3}(\mathrm{~s})$. The approximations are carried out by simply ignoring the time delay in each of the transfer functions. This procedure leads to three PID controllers. The controller in $100 \mathrm{p} 4$ is selected by approximating the pure dead-time in $\mathrm{g}_{44}(\mathrm{~s})$ with a first order Pade. In this case, the IMC tuning rules lead to a PI controller augmented with a first order lag.

"Filter time constants" $\varepsilon_{i}=15 \mathrm{~min},(i=1,4)$, ensure that each of the transfer function $\tilde{h}_{i}(s),(i=1,4)$, is stable and has its magnitude $\left|\tilde{h}_{i}(j \omega)\right|$, $(i=1,4)$, bounded by $H^{-1}(E(j \omega)$ ) (lines $1-5$ in Fig. 6, respectively). The corresponding controller parameters are shown in Table 4.

Closed-10op responses to unit step changes in $r_{i}(s),(i=1,4)$, are shown in Figs. 7-10, respectively. The effect of the interactions is evidently pronounced, especially in the case of Fig. 9. Figures 11 and 12 show responses to step changes in $r_{2}(1)$ and $r_{3}(s)$, respectively, when the closed-loop time constants have been increased to $\varepsilon_{i}=45 \mathrm{~min}$, $(i=1,4)$, and the controller parameters have been adjusted accordingly. The increased time constants improve the response shown in Fig. 11 but not that shown in Fig. 12. In general, increasing the margin between 
$\left|\tilde{h}_{i}(j \omega)\right|$ and $\mu^{-1}(E(j \omega))$ will increase the sluggishness of the closed-1oop response but will not necessarily improve its performance. These results confirm the earlier claim that Theorem 1 guarantees the stability of $\mathrm{H}(\mathrm{s})$ but that performance can be poor. The selection of appropriate "filter time constants" for each loop remains, as of this moment, an open problem.

In this particular case, the large deviation displayed by $\mathrm{y}_{4}$ suggests the controller in loop 4 is insufficiently tight and that the overall response might be improved if the time constant $\varepsilon_{4}$ is reduced while $\varepsilon_{i}$, $(i=1,3)$, remain at a fixed value of $45 \mathrm{~min}$. The extent to which $\varepsilon_{4}$ can be reduced, however, is limited to approximately $\varepsilon_{4}=10$ min, on basis of Theorem 4. Smaller values of $\varepsilon_{4}$ result in a plot of $\left|\tilde{h}_{4}(j \omega)\right|$ which violates the constraint set by $\mu^{-1}(E(j \omega))$ (Line 5 in Fig. 6). In what follows, we shall show that values of $\varepsilon_{4}$ smaller than 10 min are actually possible if we account for the large margins which exist between $\left|\tilde{h}_{j}(j \omega)\right|, \quad(i=1,3)$, and $\mu^{-1}(E(j \omega))$. Intuitively, it is reasonable to expect that these margins will allow us to relax the constraint set on $\left|\tilde{h}_{4}(j \omega)\right|$. This, in fact, is the motivation for introducing weighting matrices into the $\mu$-constraint as in Eq. (30). Such matrices can account for the fact that $\left|\tilde{h}_{i}(j \omega)\right|,(i=1,3)$, roll of $f$ at a frequency smaller than that imposed by the interactions, and use this to establish a less conservative bound on $\left|\tilde{h}_{4}(j \omega)\right|$. In effect, a weighting matrix allows us to "distribute" the margins among the $\tilde{h}_{i}(s)$.

Let us therefore introduce the weighting matrix

$$
\mathbf{W}(s)=\operatorname{diag}\left[\frac{1}{(45 s+1)}, \frac{1}{(45 s+1)}, \frac{1}{(45 s+1)}, 1\right]
$$

into the $\mu$-constraint. Equation (30) now becomes our sufficient 
condition for stability. The transfer functions

$$
w_{i}(s)=\frac{1}{(45 s+1)}, i=1,3
$$

in (40) express the fact that $\left|\tilde{h}_{i}(j \omega)\right|,(i=1,3)$, roll off as first order lags at a time constant $\varepsilon_{i}=45 \mathrm{~min},(i=1,3)$. The fact that $\mathrm{w}_{4}(\mathrm{~s})$ was assigned a value of unity simply implies that no constraint has been placed on the value of $\varepsilon_{4}$. Therefore for loop 4, (30) reduces to

$$
\left|\tilde{h}_{4}(j \omega)\right|<\mu^{m-1}(E(j \omega) W(j \omega)) \quad \forall \omega
$$

In view of (40), the constraint set by (42) can only be less conservative than that set by (20), or,

$$
\left|\tilde{h}_{4}(j \omega)\right|<\mu^{-1}(E(j \omega)) \quad \forall \omega
$$

The reduced conservatism of (42) is confirmed by the plot of $\mu^{-1}(E(j \omega) W(j \omega))$ (Line 5 in Fig. 13). Compare with Line 5 in Fig. 6.

Lines $1-3$ in Fig. 13 show plots of $\left|\tilde{h}_{i}(j \omega) w_{i}^{-1}(j \omega)\right|, \quad(i=1,3)$, respectively while Line 4 shows a plot of $\left|\tilde{h}_{4}(j \omega)\right|$ when $\varepsilon_{4}=2$ min. The controller parameters corresponding to these filter time constants are shown in Table 5. The plot of $\mu^{-1}(E(j \omega) \mathbf{W}(j \omega)$ ) (Line 5 in Fig. 13) shows that (30) is satisfied and therefore the complete closed loop system will be stable. Figures 14 and 15 show the response of the closed-1oop system for unit step changes in $r_{2}(s)$ and $r_{3}(s)$. The improved responses justify the smaller closed loop time constant in $100 \mathrm{p} 4$.

Example 3. This last example will illustrate the usefulness of Corollary 1.2. We consider a $2 x 2$ system with transfer matrix

$$
G(s)=\left[\begin{array}{cc}
\frac{5}{(4 s+1)} & \frac{2.5 e^{-5 s}}{(2 s+1)(15 s+1)} \\
\frac{-4 e^{-6 s}}{(s+1)(20 s+1)} & \frac{1}{(3 s+1)}
\end{array}\right]
$$


under decentralized control. For a decentralized controller with pairings $((1,1),(2,2)), \kappa(0)=-2$, whereas for pairings $((1,2),(2,1)) \kappa(0)$ $=-0.5$. Corollary 3.1 suggests that the decentralized controller should be implemented on these latter pairings in order to allow integral action on both control loops. This choice, however, appears undesirable because of the time delays and relatively slow dynamics associated with $g_{12}(s)$ and $g_{21}(s)$. In order to investigate the feasibility and desirability of the pairings $((1,1),(2,2))$ the necessary and sufficient stability condition of Cor. 1.2 has to be utilized rather than the conservative sufficient condition of Cor. 4.1 .

We first consider a control structure based on the diagonal transfer functions $g_{11}(s)$ and $g_{22}(s)$. Figure 16 shows the Bode plot of $k(s)$ for this structure (Line 1). Since encirclement of the point $(1,0)$ is easily avoided, it should be possible to design controllers giving rise to two closed-1oop transfer functions $\tilde{h}_{1}(s)$ and $\tilde{h}_{2}(s)$ such that $z(s)=$ $\tilde{h}_{1}(s) \tilde{h}_{2}(s)_{K}(s)$ also avoids the encirclement. Applying the IMC tuning rules for first-order lags to $g_{11}(s)$ and $g_{22}(s)$ yields two controllers with proportional-integral action. The controller parameters corresponding to a unit value of $\varepsilon$ are shown in Table 5. With these parameters, the Bode plot of $z(s)$ (Line 2 in Fig. 16) is not significantly different from that of $k(s)$ and stability of the $2 \times 2$ system is guaranteed. The closed-10op response of the system for a unit step change in $r_{1}(s)$ is shown in Fig. 17.

Next we consider a control structure based on the off-diagonal transfer functions $g_{12}(s)$ and $g_{21}(s)$. Line 3 in Fig. 18 is a plot of $\mu^{-1}(E(j \omega))$ for these pairings. To select controllers for $g_{12}(s)$ and $g_{2 i}(s)$, we approximate these transfer functions as first order lags with dead- 
time and apply the IMC tuning rules. The approximation is performed by ignoring the smaller of the two time constants in $g_{12}(s)$ and $g_{21}(s)$. The tuning rules for first order lag with dead-time yield PID controllers. Controller parameters corresponding to a filter constant $\varepsilon=15$ are shown in Table 5. This value of $\varepsilon$ was selected to ensure that the closed-loop transfer functions $\tilde{h}_{1}(s)$ and $\tilde{h}_{2}(s)$ corresponding to $g_{12}(s)$ and $g_{21}(s)$ (Lines 1 and 2 in Fig. 18) lie within the constraint set by $\mu^{-1}(E(j \omega))$. The closed-1oop response of the system for a unit step change in $r_{1}(s)$ is shown in Fig. 19. The better response shown in Fig. 17 justifies the diagonal pairings based on Cor. 4.1.

This example shows that it is useful to consider both the magnitude and the phase of $\kappa(s)$ for variable pairing and controller design in $2 \times 2$ systems. It also shows that the performance of these systems can be quite acceptable when $\kappa<-1 \quad(\lambda<1 / 2)$. In general, for $2 \times 2$ systems, variables should be paired such that $|\kappa(0)|<1$ because of Corollary 4.1 . However, if $\kappa(0)<-1$ but $|\kappa(j \omega)|$ is a decreasing function of frequency with a slope between -1 and -2 , this pairing may often be preferable as illustrated in Example 3.

\section{CONCLUSION}

The purpose of an "Interaction Measure" is to quantify the performance degradation caused by a decentralized control structure. More specifically, an Interaction Measure was defined to be a bound on the magnitude of the decentralized closed-loop transfer matrix $\tilde{\mathrm{H}}$ (s) sufficient to guarantee the stability of the full closed-loop transfer $\operatorname{matrix} \mathrm{H}(\mathrm{s})$. 
Using the notion of Structured Singular Value, the "optimal" dynamic Interaction Measure was derived which provides the tightest bound which can be established on $\sigma_{\max }(\tilde{\mathrm{H}}(j \omega))$. This measure, called the $\mu$ Interaction Measure is a unique function of the "relative error matrix" $E(s)=(G(s)-\tilde{G}(s)) \tilde{G}^{-1}(s)$, and the structure of the decentralized controller.

The $\mu$ Interaction Measure treats multivariable systems of arbitrary size under feedback with diagonal or block-diagonal controllers, all in a unified manner. it can be conveniently displayed on an amplitudefrequency diagram to predict the stability of decentralized control systems and to measure the performance loss caused by these control structures. In particular, its steady-state value provides a sufficient condition for achieving offset-free performance. In the case of decentralized $2 \times 2$ systems, the $\mu$ Interaction Measure is closely related to Rijnsdorp's Interaction Measure.

\section{ACKNOWLEDGMENTS}

Support from the Department of Energy and the National Science Foundation are gratefully acknowledged. We are thankful to J. Doyle and S. Skogestad for many useful discussions. 


\section{APPENDIX}

Proof of Corollary 1.3: Because of assumptions (i) and (ii) (34) becomes

$$
z(s)=\tilde{h}_{1}(s) k
$$

in the frequency range of interest. In the following $\omega^{*}$ will denote all frequencies for which

$$
\operatorname{Im}\left\{g_{11} \mathrm{c}_{1}\left(j \omega^{*}\right)\right\}=0
$$

Case A: $\lambda>1,0<\kappa<1$

From Cor. 1.2 and (A1) $\mathrm{H}(\mathrm{s})$ is stable if

$$
\tilde{h}_{1}\left(j \omega^{*}\right)=\frac{g_{11} c_{1}\left(j \omega^{*}\right)}{1+g_{11} c_{1}\left(j \omega^{*}\right)}<\frac{1}{k}
$$

(A3) is always satisfied because $g_{1} c_{1}\left(j \omega^{*}\right)>-1$ by assumption (iii).

Case B: $0<\lambda<1$, $\quad k<0$

Detuning $c_{1}(s)$ by a factor $\lambda$ implies that for the multivariable system

$$
c_{1}^{\prime}(s)=\lambda c_{1}(s)
$$

From Cor. 1.2 and (A1) H(s) is stable if

$$
\kappa \tilde{h} !\left(j \omega^{*}\right)=\kappa \frac{\lambda g_{11} c_{1}\left(j \omega^{*}\right)}{\left.1+\lambda g_{11} c_{1}(j \omega)^{*}\right)}<1
$$

For $\tilde{h}_{1}^{\prime}\left(j \omega^{*}\right)>0(A 5)$ is always satisfied. For $\tilde{h} !\left(j \omega^{*}\right)<0$ (A5) is equivalent to

$$
|k|\left|\tilde{h}_{i}(j \omega)^{*}\right|<1
$$

$\tilde{h}:\left(j \omega^{*}\right)<0$ if $-1<\lambda g_{1_{1}} c_{1}\left(j \omega^{*}\right)<0$. Because of (iii) $g_{11} c_{1}\left(j \omega^{*}\right)>-1$ and therefore $(A 6)$ has to be satisfied for $-1<g_{11} c_{1}\left(j \omega^{*}\right)<0$. For this range of values (A6) can be rewritten as

$$
\begin{aligned}
& \left|\frac{\lambda-1}{\lambda}\right| \frac{\mid \lambda g_{11} c_{1}\left(j \omega^{*}\right)}{\left|+\lambda g_{11} c_{1}\left(j \omega^{*}\right)\right|}< \\
& \left|\frac{\lambda-1}{\lambda}\right| \frac{\lambda}{\left|1+\lambda g_{11} c_{1}\left(j \omega^{*}\right)\right|}=
\end{aligned}
$$




$$
\begin{gathered}
\left|\frac{\lambda-1}{\lambda}\right| \frac{\lambda}{1-\lambda \mid g_{11} c_{1}\left(j \omega^{*}\right)} \mid< \\
\left|\frac{\lambda-1}{\lambda}\right| \frac{\lambda}{1-\lambda}=1
\end{gathered}
$$


REFERENCES

Bristol, E. H. (1966). On a New Measure of Interaction for Multivariable Process Control. IEEE Trans. Autom. Control, AC-11, 133-134. Davison, E. J. (1970). Interaction Index for Multivariable Control Systems, IEE Proc., 117, 459-462.

Doukas, N. and W. Luyben (1978). Control of Sidestream Columns Separating Ternary Mixtures. Anal. Instrum., 16, 51-58. Doyle, J. (1982). Analysis of Feedback Systems with Structured Uncertainties. IEE Proc., 129, 242-250.

Gagnepain, J. P. and D. E. Seborg (1982). Analysis of Process Interactions with Applications to Multiloop Control System Design. Ind. Eng. Chem. Process Des. Dev., 21, 5-11. Grosdidier, P., M. Morari and B. R. Holt (1985). Closed-Loop Properties from Steady-State Gain Information. Ind. Eng. Chem. Fund., 24, $221-235$.

Grosdidier, P. and M. Morari (1985). Interaction Measures for Systems

Under Decentralized Control. Accepted for publication, Automatica.

Jensen, N. (1985). Letter to the Editor. Ind. Eng. Chem. Process Des. Dev., 24, 228-229.

Jensen, N., D. G. Fisher and S. L. Shah (1985). Interaction Analysis in Multivariable Control System Design. AIChE J. (in press). Limebeer, D. J. N. (1982). The Application of Generalized Diagonal Dominance to Linear System Stability Theory. Int. J. Control, 36, $185-212$

McAvoy, T. J. (1981). Connection Between Relative Gain and Control Loop 
Stability and Design. AIChE J., 27, 613-619.

McAvoy, T. J. (1985). Letter to the Editor. Ind. Eng. Chem. Process Des. Dev., 24 229-230.

Mees. A. I. (1981). Achieving Diagonal Dominance. Systems and Control Letters, 1, 155-158.

Morari, M. (1985). Robust Stability of Systems with Integral Control. IEEE Trans. Autom. Control, AC-30, 574-577.

Osborne, E. E. (1960). On Pre-Conditioning of Matrices. J. Assoc. Comput. Mach., 7, 338-345.

Rijnsdorp, J. E. (1965). Interaction in Two-Variable Control Systems for Distillation Columns - I. Automatica, 1, 15-28.

Rivera, D. E., M. Morari and S. Skogestad (1986). Internal Model Control. 4. PID Controller Design. Ind. Eng. Chem. Process Des. Dev, 25, $252-265$

Rosenbrock, H. H. (1974). Computer-Aided Control System Design. Academic Press, London.

Shinskey, F. G. (1979). Process Control Systems. McGraw-Hill Book Co., New York.

Tung, L. S. and T. F. Edgar (1981). Analysis of Control-output Interactions in Dynamic Systems. AIChE J., 28, 590-693. Witcher, M. F. and T. J. McAvoy (1977). Interacting Control Systems: Steady State and Dynamic Measurement of Interaction. ISA Trans., $16,34-41$

Woolverton, P. F. (1980). How to Use Relative Gain Analysis in Systems with Integrating Variables. InTech, September, 63-65. 
Table 1. Distillation Column Transfer Function Matrix

\begin{tabular}{|c|c|c|c|c|}
\hline & $u_{1}$ & $u_{2}$ & $u_{3}$ & $u_{4}$ \\
\hline $\mathrm{y}_{1}$ & $\frac{-11 \cdot 3 e^{-3.79 s}}{(21.74 s+1)^{2}}$ & $\frac{0.374 e^{-7.75 s}}{(22.22 s+1)^{2}}$ & $\frac{-9.811 e^{-1.59 s}}{(11.36 s+1)}$ & $\frac{-2.37 e^{-27.33 s}}{(33 \cdot 3 s+1)}$ \\
\hline $\mathrm{y}_{2}$ & $\frac{5.24 e^{-60 s}}{(400 s+1)}$ & $\frac{-1.986 e^{-0.71 s}}{(66.67 s+1)^{2}}$ & $\frac{5.984 e^{-2.24 s}}{(14.29 s+1)}$ & $\frac{0.422 e^{-8.72 s}}{(250 s+1)^{2}}$ \\
\hline $\mathrm{y}_{3}$ & $\frac{-0.33 e^{-0.68 s}}{(2.38 s+1)^{2}}$ & $\frac{0.0204 e^{-0.59 s}}{(7.14 s+1)^{2}}$ & $\frac{2.38 e^{-0.42 s}}{(1.43 s+1)^{2}}$ & $0.513 e^{-s}$ \\
\hline $\mathrm{y}_{4}$ & $\frac{4.48 e^{-0.52 s}}{(11.11 s+1)}$ & $\frac{-0.176 e^{-0.48 s}}{(6.90 s+1)^{2}}$ & $\frac{-11.67 e^{-1.91 s}}{(12.19 s+1)}$ & $15.54 e^{-s}$ \\
\hline
\end{tabular}

Table 2. Controlled and Manipulated Variables

$\begin{array}{ll}\mathrm{y}_{1}=\text { Toluene impurity in the bottom } & \mathrm{u}_{1}=\text { Sidestream flowrate } \\ \mathrm{y}_{2}=\text { Toluene impurity in the distillate } & \mathrm{u}_{2}=\text { Reflux ratio } \\ \mathrm{y}_{3}=\text { Benzene impurity in the sidestream } & \mathrm{u}_{3}=\text { Reboil duty } \\ \mathrm{y}_{4}=\text { Xylene impurity in the sidestream } & \mathrm{u}_{4}=\text { Side draw location }\end{array}$


118

Table 3. Relative Gain Array for $4 \times 4$ System in Table 1. Boxes indicate recommended pairings

\begin{tabular}{llll}
\hline 1.006 & -0.101 & 0.126 & -0.030 \\
-0.104 & 1.094 & 0.011 & 0 \\
0.108 & 0.002 & 0.723 & 0.166 \\
-0.010 & 0.005 & 0.140 & 0.864 \\
\hline
\end{tabular}


Table 4: PD Controller Parameters for Example 2

\begin{tabular}{|c|c|c|c|c|}
\hline & $c_{1}(s)$ & $c_{2}(s)$ & $c_{3}(s)$ & $c_{4}(s)$ \\
\hline \multicolumn{5}{|c|}{ Initial Tuning Parameters } \\
\hline$\varepsilon_{i}, \min$ & 15 & 15 & 15 & 15 \\
\hline$k_{i}$ & -0.26 & -4.5 & 0.080 & 0.0020 \\
\hline $1 / \tau_{i}, \min ^{-1}$ & 0.023 & 0.0075 & 0.35 & 2 \\
\hline$\tau_{D}, \min$ & 10.9 & $33 \cdot 3$ & 0.715 & 0 \\
\hline$\tau_{F}, \min$ & 0 & 0 & 0 & 0.47 \\
\hline \multicolumn{5}{|c|}{ Final Tuning Parameters } \\
\hline$\varepsilon_{i}, \min$ & 45 & 45 & 45 & 2 \\
\hline$k_{i}$ & -0.085 & -1.5 & 0.027 & 0.011 \\
\hline $1 / \tau_{i}, \min ^{-1}$ & 0.023 & 0.0075 & 0.35 & 2 \\
\hline${ }^{\mathrm{T}} \mathrm{D}, \min$ & 10.9 & $33 \cdot 3$ & 0.715 & 0 \\
\hline$\tau_{F}, \min$ & 0 & 0 & 0 & 0.33 \\
\hline
\end{tabular}

Note: $\tau_{F}$ is the first order lag time constant 
Table 5: PID Controller Parameters for Example 3

\begin{tabular}{|c|c|c|c|c|}
\hline & \multicolumn{2}{|c|}{ Diagonal Pairings } & \multicolumn{2}{|c|}{ Off-Diagonal Pairings } \\
\hline & $c_{1}(s)$ & $c_{2}(s)$ & $c_{1}(s)$ & $c_{2}(s)$ \\
\hline$k$ & 0.8 & 3 & 0.40 & -0.32 \\
\hline $1 / \tau_{i}, \min ^{-1}$ & 0.25 & 0.33 & 0.057 & 0.043 \\
\hline$\tau_{d}, \min$ & 0 & 0 & 2.1 & 2.6 \\
\hline
\end{tabular}




\section{FIGURE CAPTIONS}

Figure 1. General decentralized control structure.

Figure 2A. Decentralized control structure for a $2 \times 2$ system with interactions.

Figure 2B. Decentralized control structures for a $2 \times 2$ system without interactions.

Figure 3. Block-diagram representation of interactions as additive uncertainty.

Figure 4. (Example 1), Interaction Measures for fully-decentralized controllers. Line $1=\mu^{-1}(E(j \omega))$ for Doukas and Luyben pairings; Line $2=\mu^{-1}(E(j \omega))$ for diagonal pairings.

Figure 5. (Example 1), Lines 1 and $2=\mu^{-1}(E(j \omega)$ ) for blockdecentralized controller with pairings $((1-2,1-2),(3,3)$, $(4,4)$ ) and $((1,1),(2,2),(3-4,3-4))$, respectively; Line $3=$ $\mu^{-1}(E(j \omega))$ for fully-decentralized controller with diagonal pairings.

Figure 6. (Example 2), Lines $1-4=\left|\tilde{h}_{i}(j \omega)\right|, i=1-4$, respectively; Line $5=\mu^{-1}(\mathbf{E}(j \omega))$ for fully-decentralized controller with diagonal pairings.

Figure 7. (Example 2), Closed-loop response for unit step change in $r_{1}(\mathrm{~s}) . \quad \varepsilon_{i}=15 \mathrm{~min}, i=1,4$.

Figure 8. (Example 2), Closed-loop response for unit step change in $r_{2}(s) . \quad \varepsilon_{i}=15 \mathrm{~min}, i=1,4$.

Figure 9. (Example 2), Closed-loop response for unit step change in $r_{3}(\mathrm{~s}) . \quad \varepsilon_{i}=15 \mathrm{~min}, i=1,4$.

Figure 10. (Example 2), Closed-1oop response for unit step change in $r^{4}(\mathrm{~s}) . \quad \varepsilon_{i}=15 \mathrm{~min}, i=1,4$. 
Figure 11. (Example 2), Closed-1oop response for unit step change in $r_{2}(\mathrm{~s}) . \quad \varepsilon_{i}=45 \mathrm{~min}, i=1,4$.

Figure 12. (Example 2), Closed loop response for unit step change in $r_{3}(\mathrm{~s}) . \quad \varepsilon_{i}=45 \mathrm{~min}, i=1,4$.

Figure 13. (Example 2), Lines $1-3=\left|\tilde{h}_{i}(j \omega) w_{i}^{-1}(j \omega)\right|, \quad i=1,3$,

respectively. Line $4=\left|\tilde{h}_{4}(j \omega)\right|$. Line $5=\mu^{-1}(E(j \omega) W(j \omega))$ for fully-decentralized controller with diagonal pairings.

Figure 14. (Example 2), Closed-1oop response for unit step change in $r_{2}(\mathrm{~s}) . \quad \varepsilon_{i}=45 \mathrm{~min}, i=1,3 ; \varepsilon_{4}=2 \mathrm{~min}$.

Figure 15. (Example 2), Closed-1oop response for unit step change in $r_{3}(s) . \quad \varepsilon_{i}=45 \mathrm{~min}, i=1,3 ; \varepsilon_{4}=2 \mathrm{~min}$.

Figure 16. (Example 3), Bode plots. Line $1=k(s)$; Line $2=z(s)=$ $\tilde{h}_{1}(s) \tilde{h}_{2}(s)_{K}(s)$, diagonal pairings.

Figure 17. (Example 3), closed-1oop response for unit step change in $r_{1}(s)$, diagonal pairings.

Figure 18. (Example 3), Lines 1 and $2=\left|\tilde{h}_{i}(j \omega)\right|, i=1,2$ respectively; Line $3=\mu^{-1}(E(j \omega))$, for diagonal controller with of $f$-diagonal pairings.

Figure 19. (Example 3), closed-1oop response for unit step change in $r_{1}(s)$, off-diagonal pairings. 


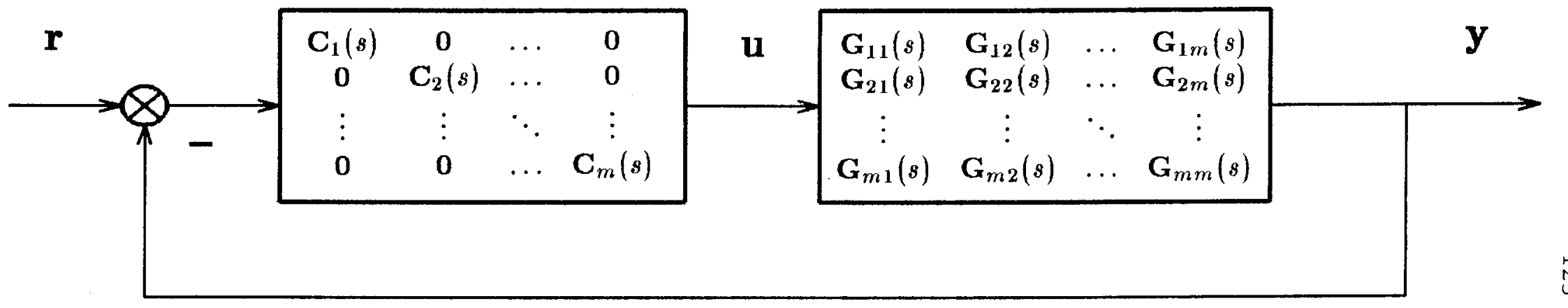

Figure 1. General decentralized control structure. 


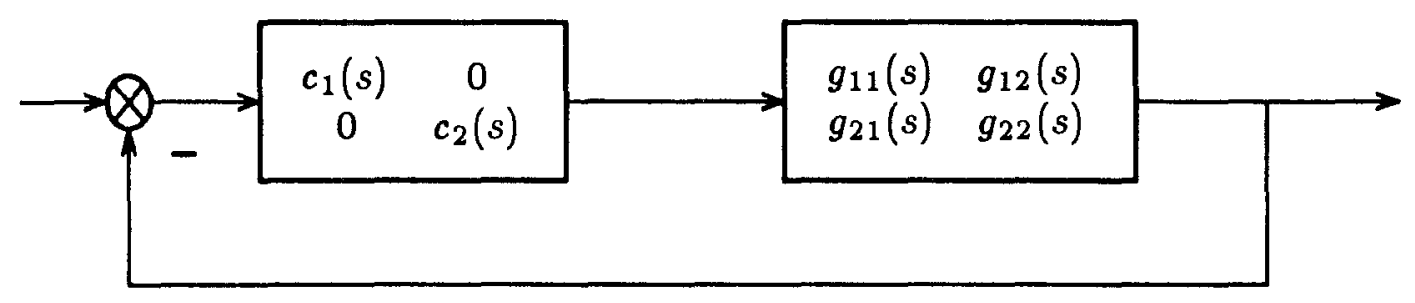

Figure 2A. Decentralized control structure for a $2 \times 2$ system with interactions.

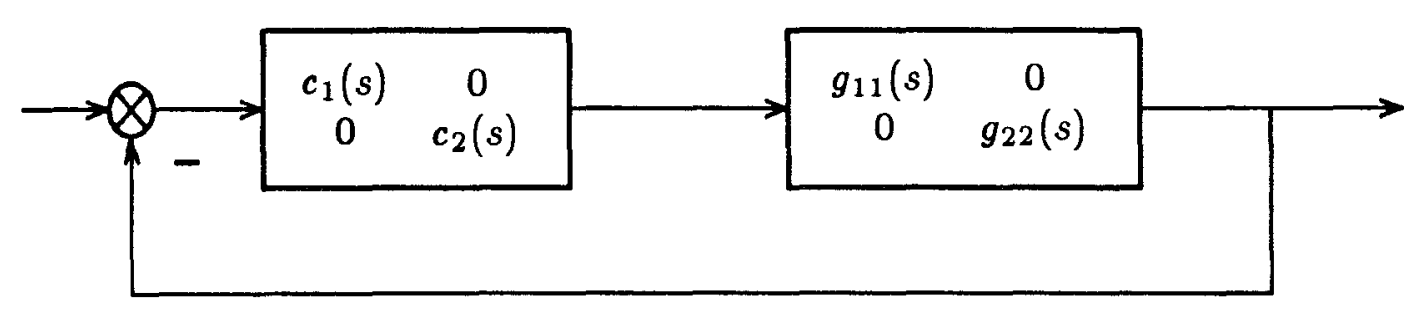

Figure 2B. Decentralized control structures for a $2 \times 2$ system without interactions. 


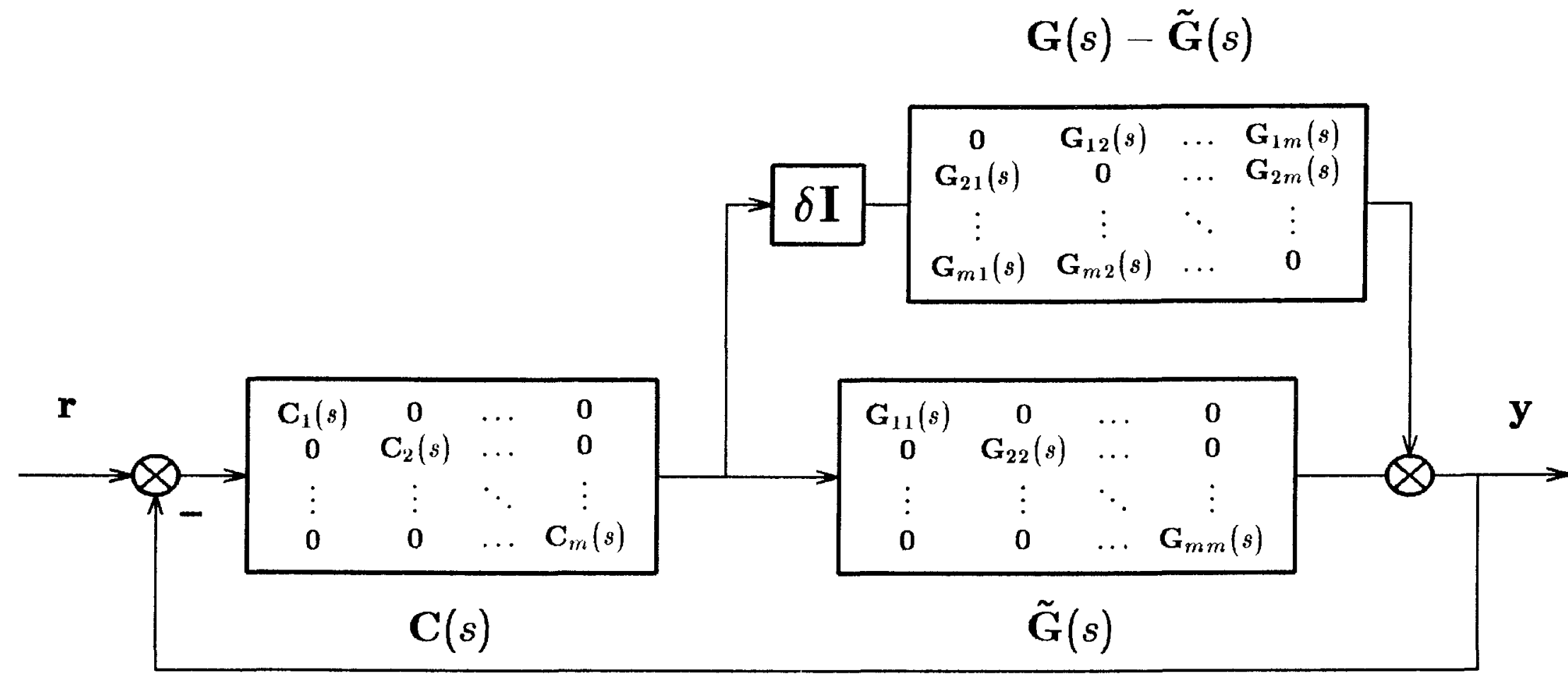

Figure 3. Block-diagram representation of interactions as additive uncertainty. 


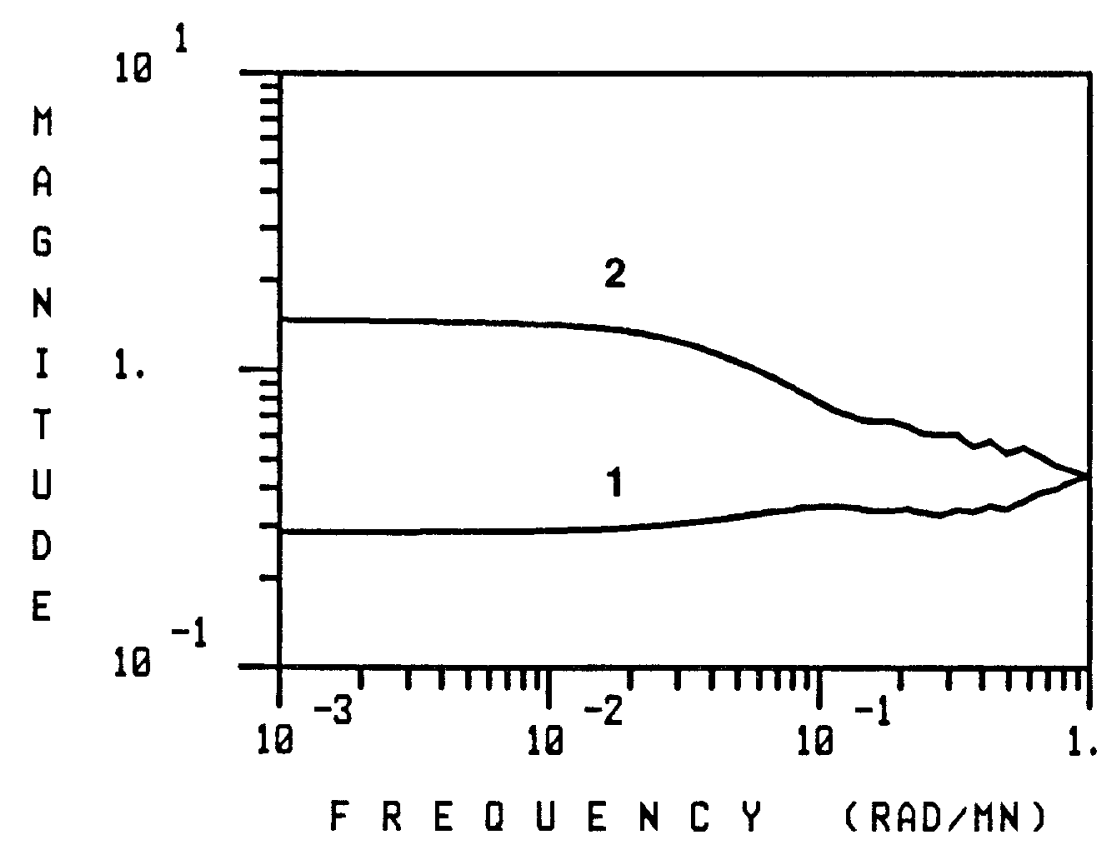

Figure 4. (Example 1), Interaction Measures for fully-decentralized controllers. Line $1=\mu^{-1}(E(j \omega))$ for Doukas and Luyben pairings; Line $2=\mu^{-1}(E(j \omega))$ for diagonal pairings. 


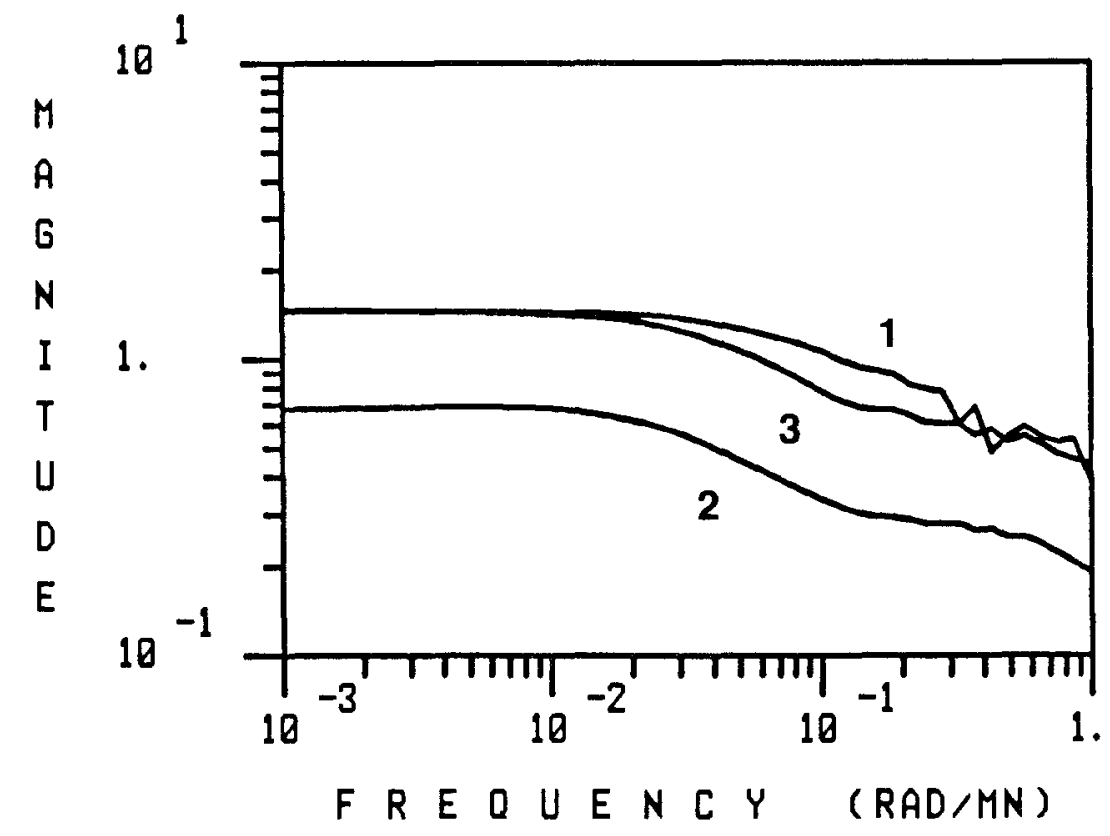

Figure 5. (Example 1), Lines 1 and $2=\mu^{-1}(E(j \omega)$ ) for blockdecentralized controller with pairings $((1-2,1-2),(3,3)$, $(4,4))$ and $((1,1),(2,2),(3-4,3-4))$, respectively; Line $3=$ $\mu^{-1}(E(j \omega))$ for fully-decentralized controller with diagonal pairings. 


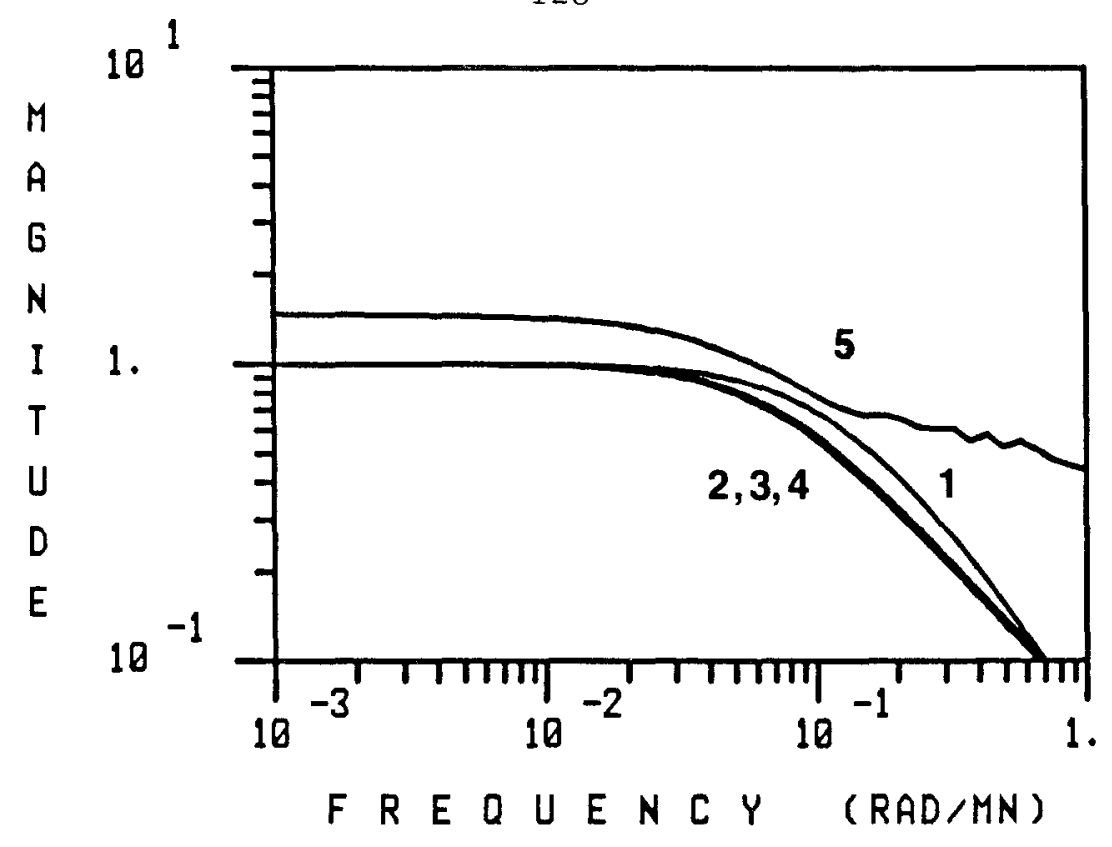

Figure 6. (Example 2), Lines $1-4=\left|\tilde{h}_{i}(j \omega)\right|, i=1-4$, respectively; Line $5=\mu^{-1}(E(j \omega))$ for fully-decentralized controller with diagonal pairings.

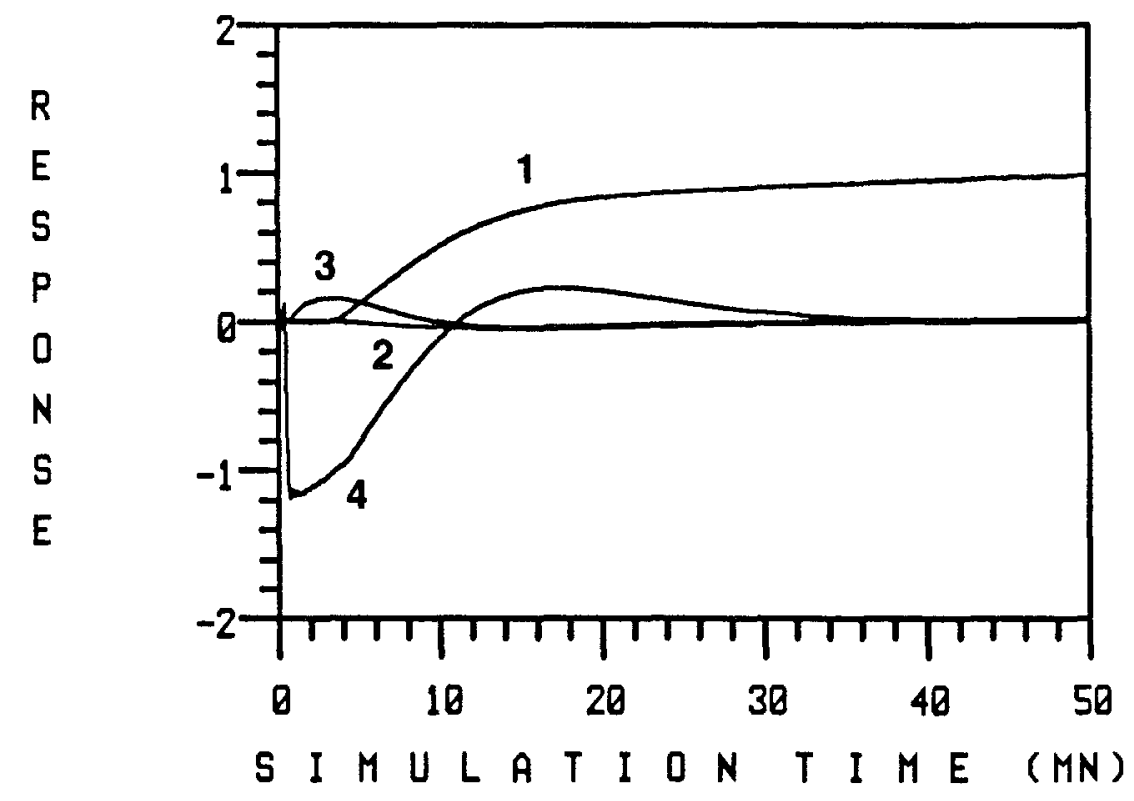

Figure 7. (Example 2), Closed-10op response for unit step change in $r_{1}(s) . \quad \varepsilon_{i}=15 \mathrm{~min}, i=1,4$. 


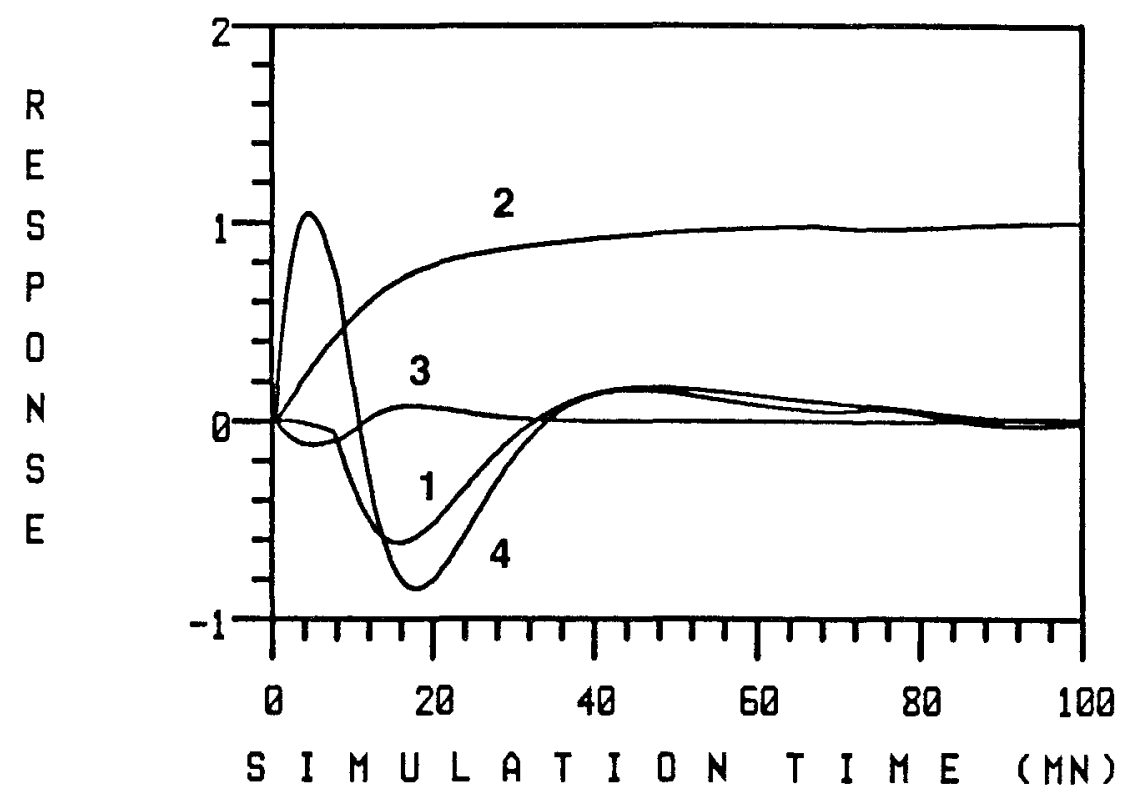

Figure 8. (Example 2), Closed-10op response for unit step change in $r_{2}(s) . \quad \varepsilon_{i}=15 \mathrm{~min}, i=1,4$.

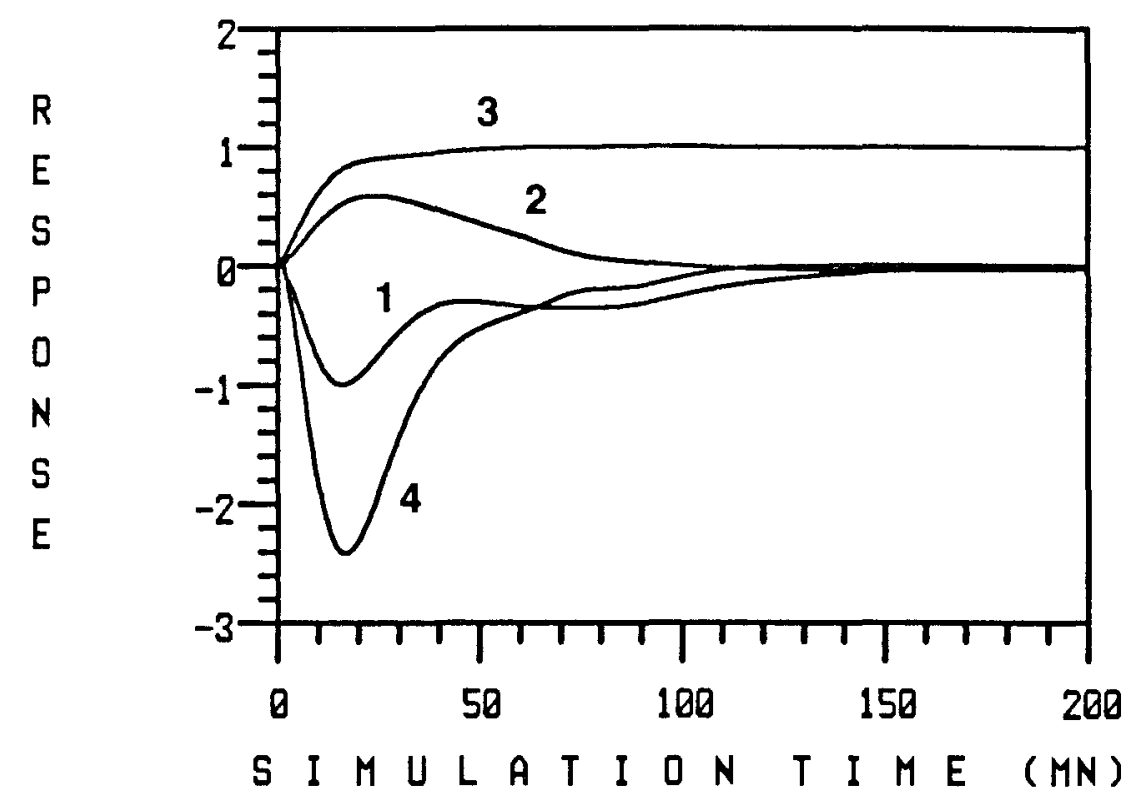

Figure 9. (Example 2), Closed-1oop response for unit step change in $r_{3}(s) . \quad \varepsilon_{i}=15 \mathrm{~min}, i=1,4$. 


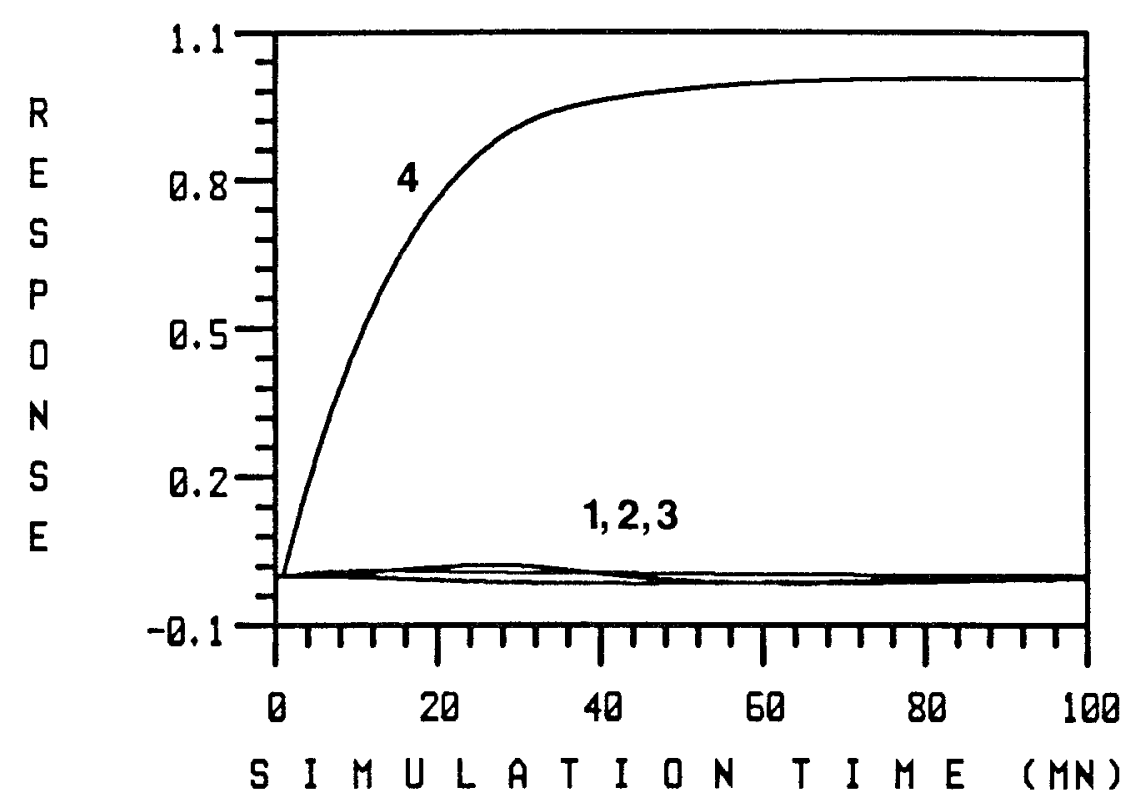

Figure 10. (Example 2), Closed-1oop response for unit step change in $r_{4}(s) . \quad \varepsilon_{i}=15 \min , i=1,4$.

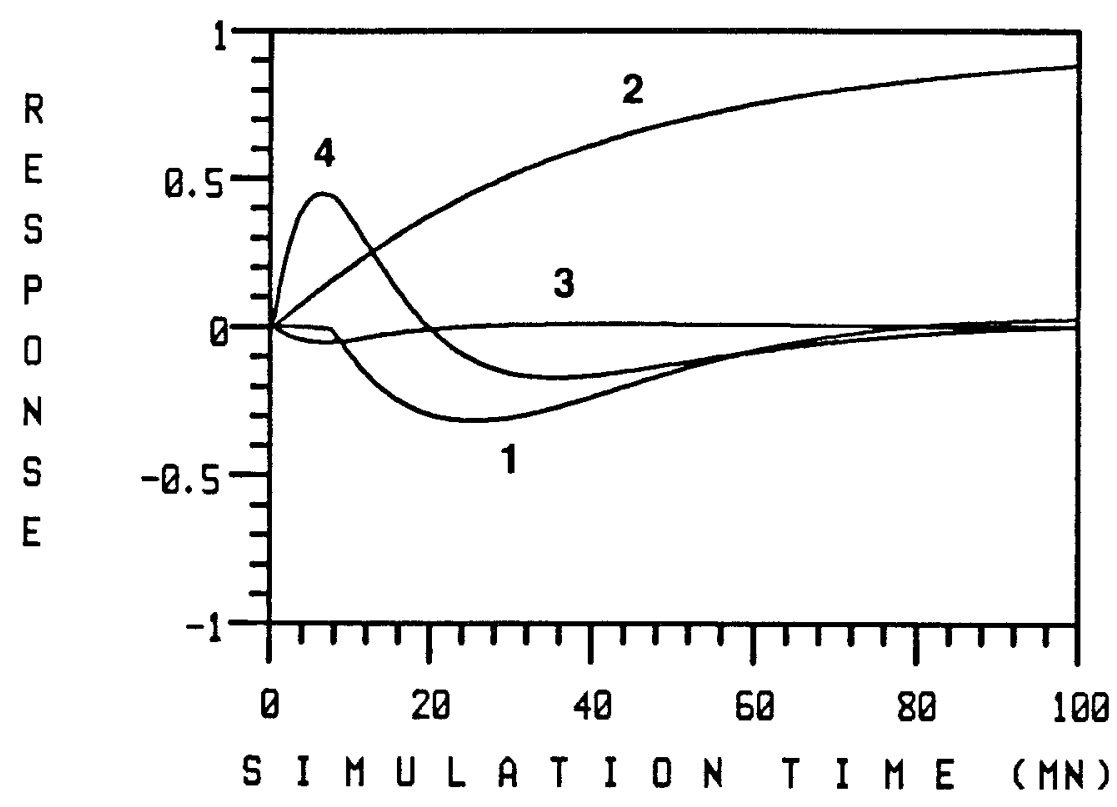

Figure 11. (Example 2), Closed-1oop response for unit step change in $r_{2}(s) . \quad \varepsilon_{i}=45 \mathrm{~min}, i=1,4$. 
131

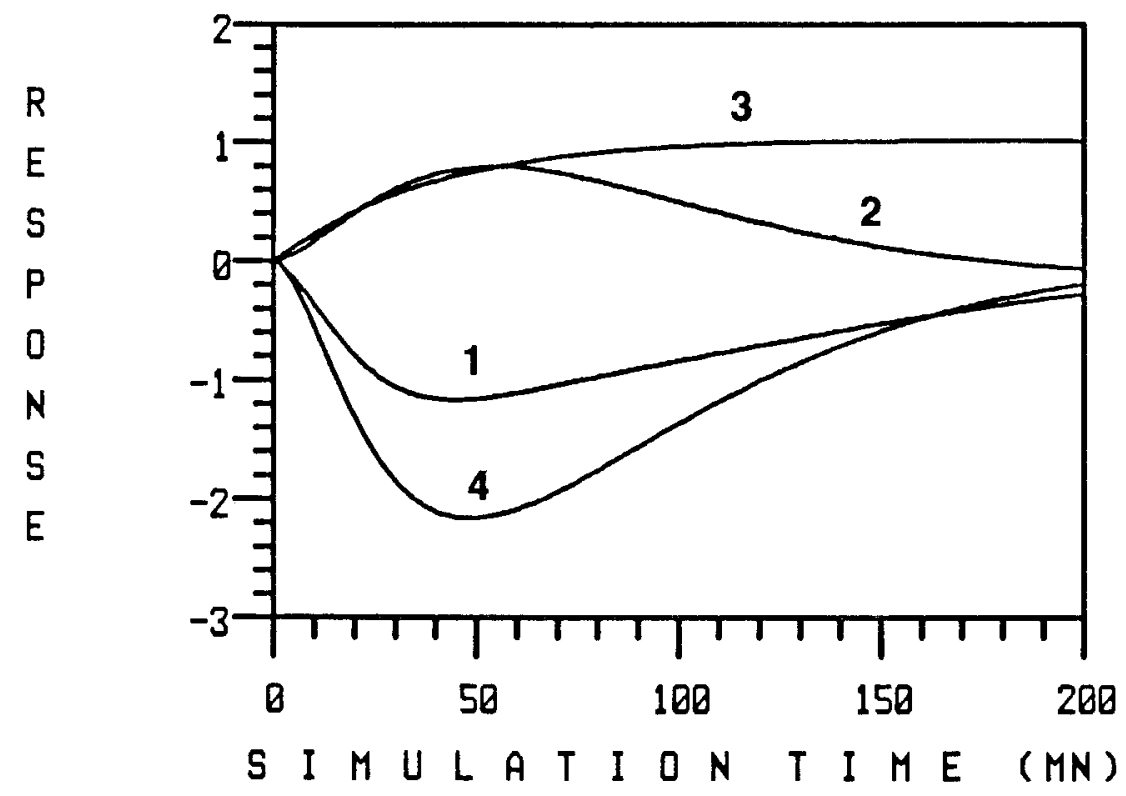

Figure 12. (Example 2), Closed loop response for unit step change in $r_{3}(\mathrm{~s}) . \quad \varepsilon_{i}=45 \mathrm{~min}, i=1,4$.

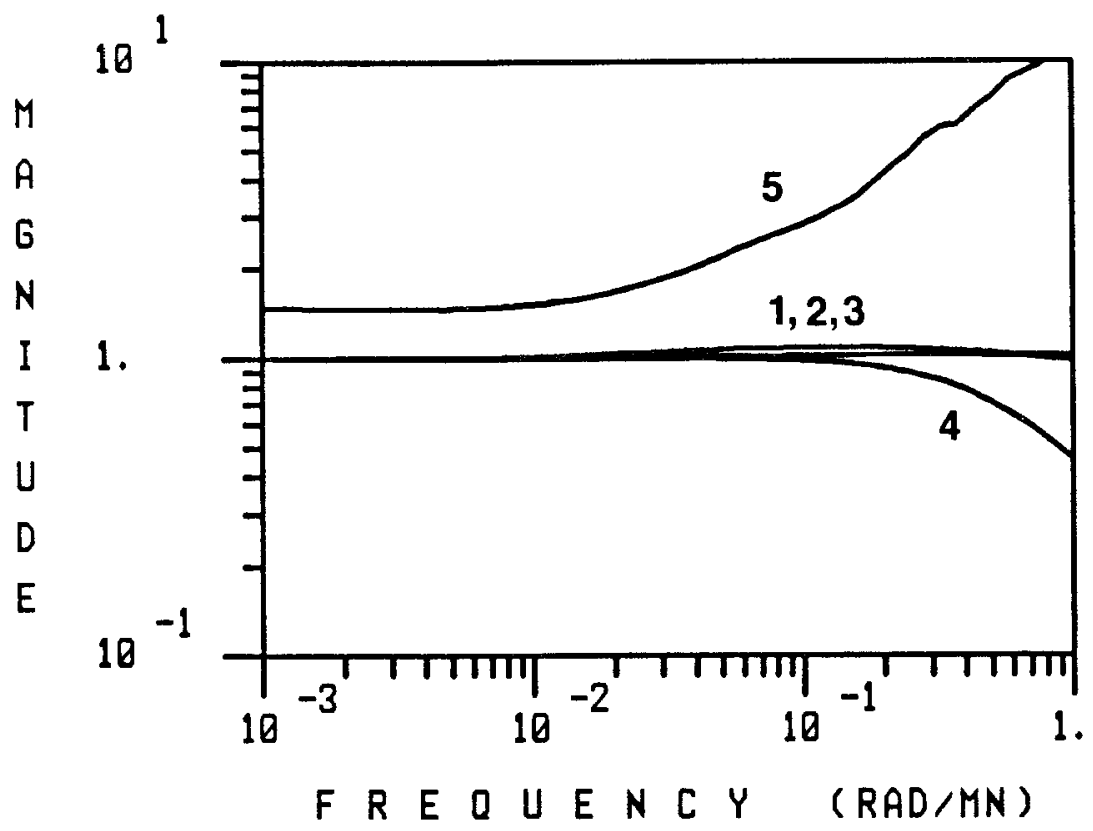

Figure 13. (Example 2), Lines $1-3=\left|\tilde{h}_{i}(j \omega) w_{i}^{-1}(j \omega)\right|, \quad i=1,3$, respectively. Line $4=\left|\tilde{h}_{4}(j \omega)\right|$. Line $5=\mu^{-1}(E(j \omega) W(j \omega))$ for fully-decentralized controller with diagonal pairings. 


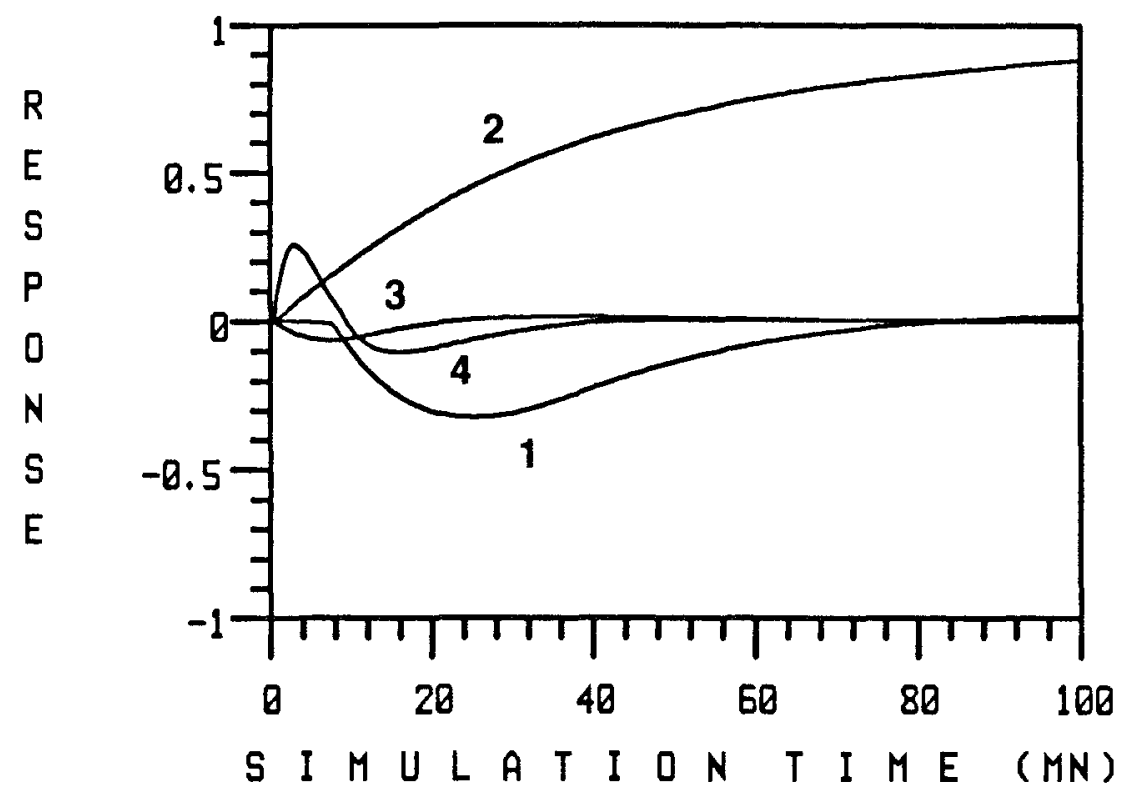

Figure 14. (Example 2), Closed-1oop response for unit step change in $r_{2}(s) . \quad \varepsilon_{i}=45 \mathrm{~min}, i=1,3 ; \varepsilon_{4}=2 \mathrm{~min}$.

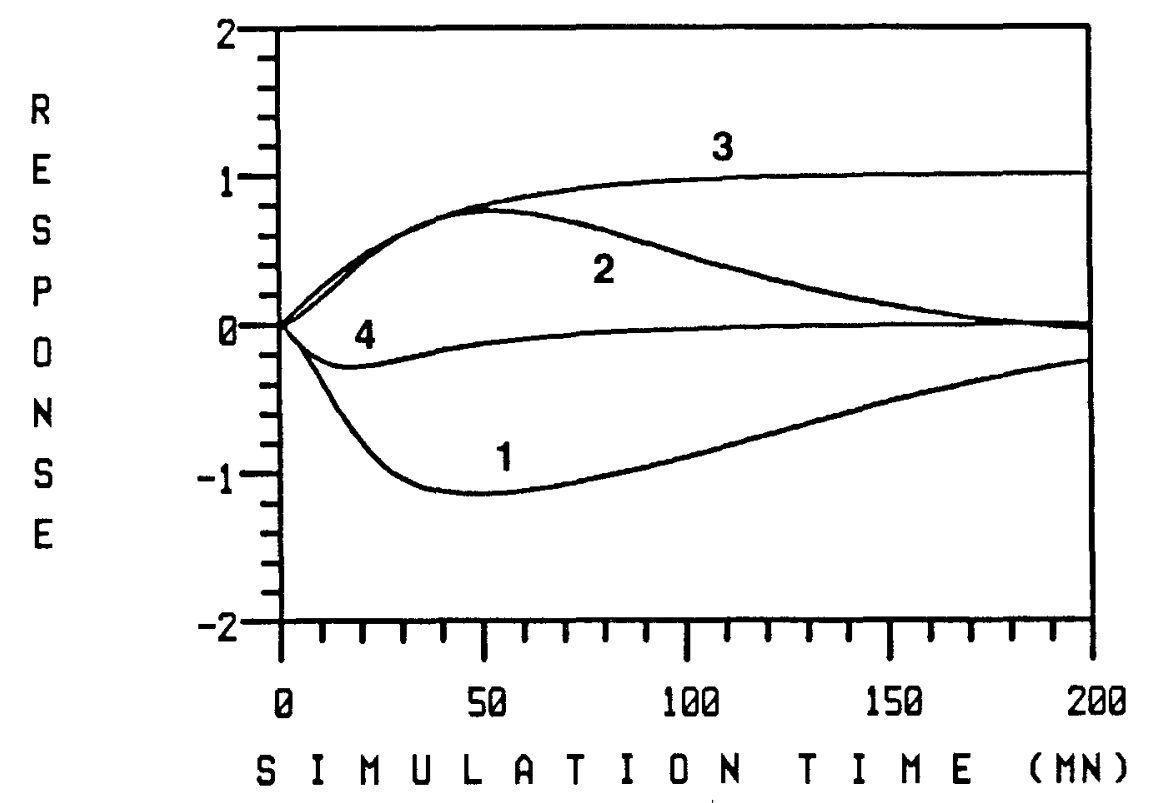

Figure 15. (Example 2), Closed-1oop response for unit step change in $r_{3}(s) . \quad \varepsilon_{i}=45 \mathrm{~min}, i=1,3 ; \varepsilon_{4}=2 \mathrm{~min}$. 

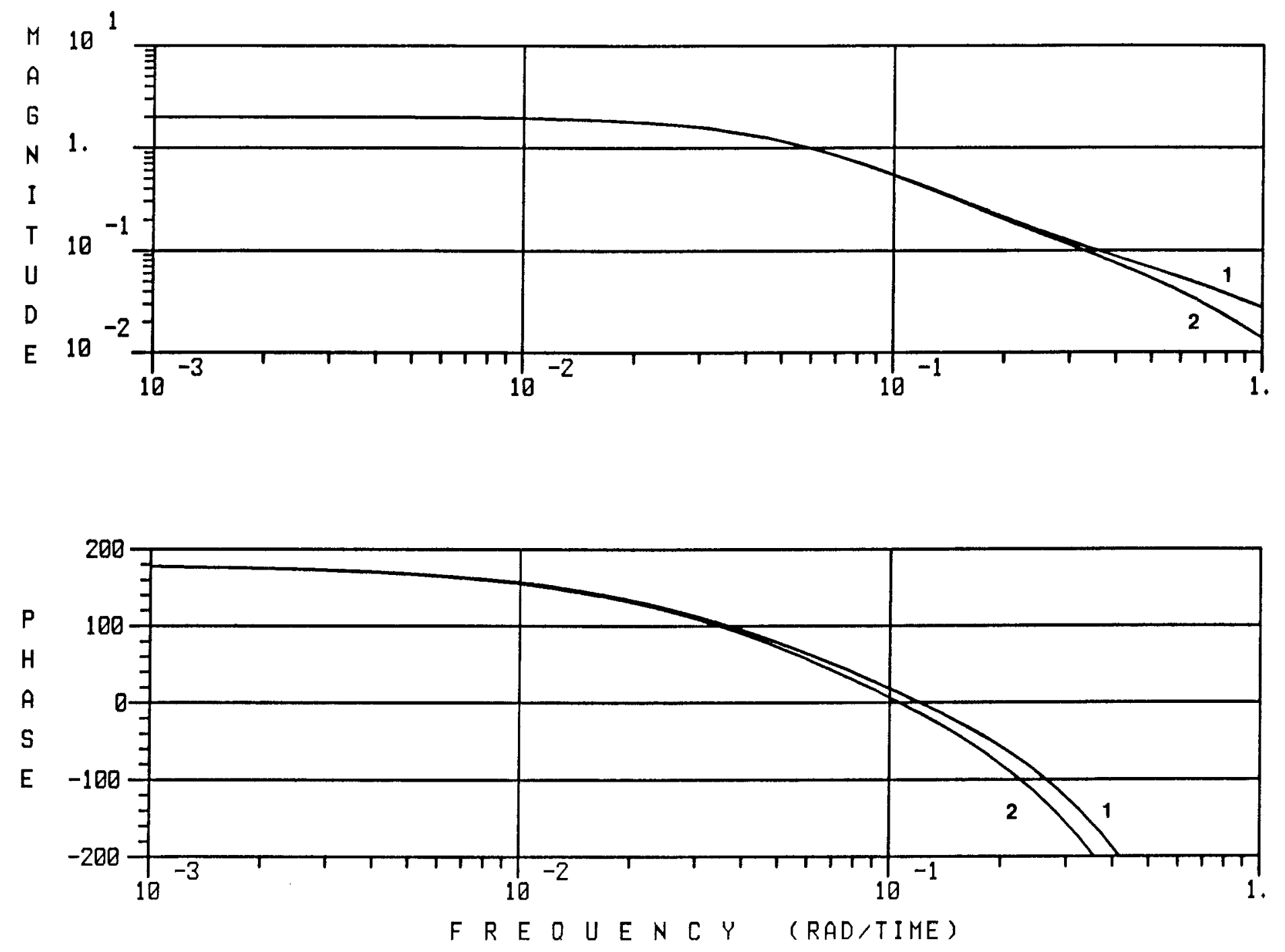

Figure 16. (Example 3), Bode plots. Line $1=\kappa(s)$; Line $2=z(s)=\tilde{h}_{1}(s) \tilde{h}_{2}(s) \kappa(s)$, diagonal pairings. 


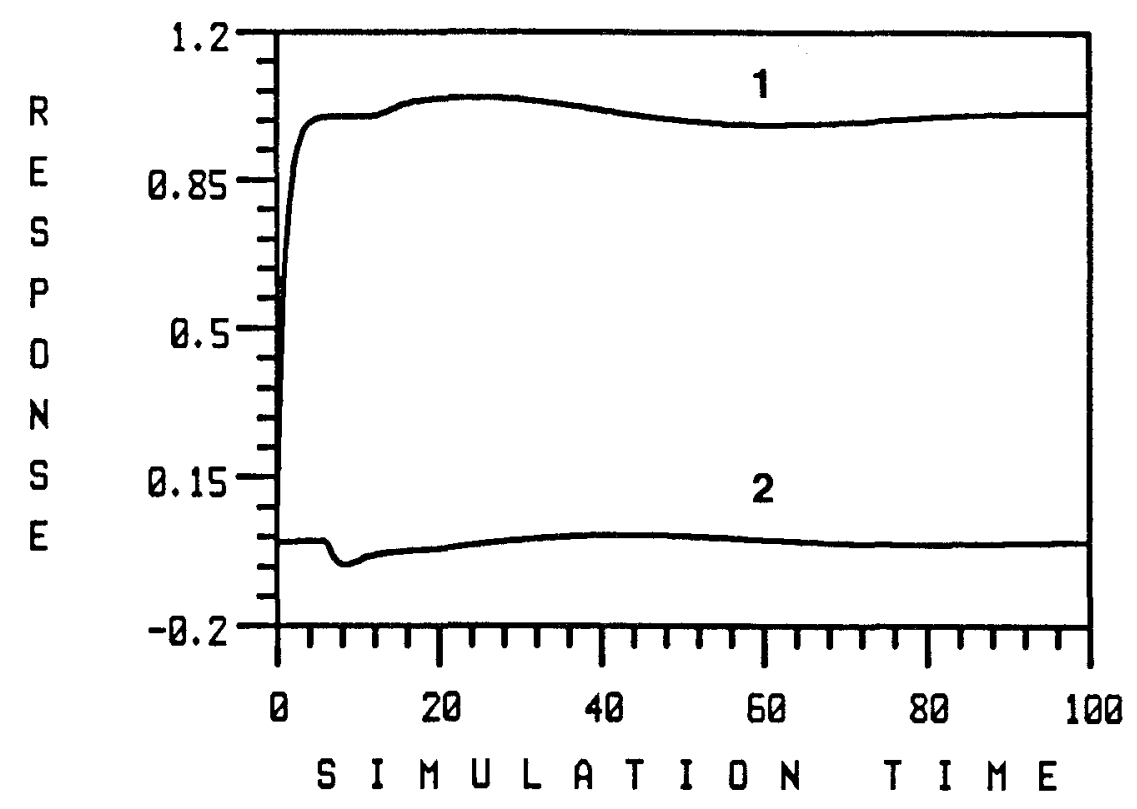

Figure 17. (Example 3), closed-loop response for unit step change in $r_{1}(s)$, diagonal pairings.

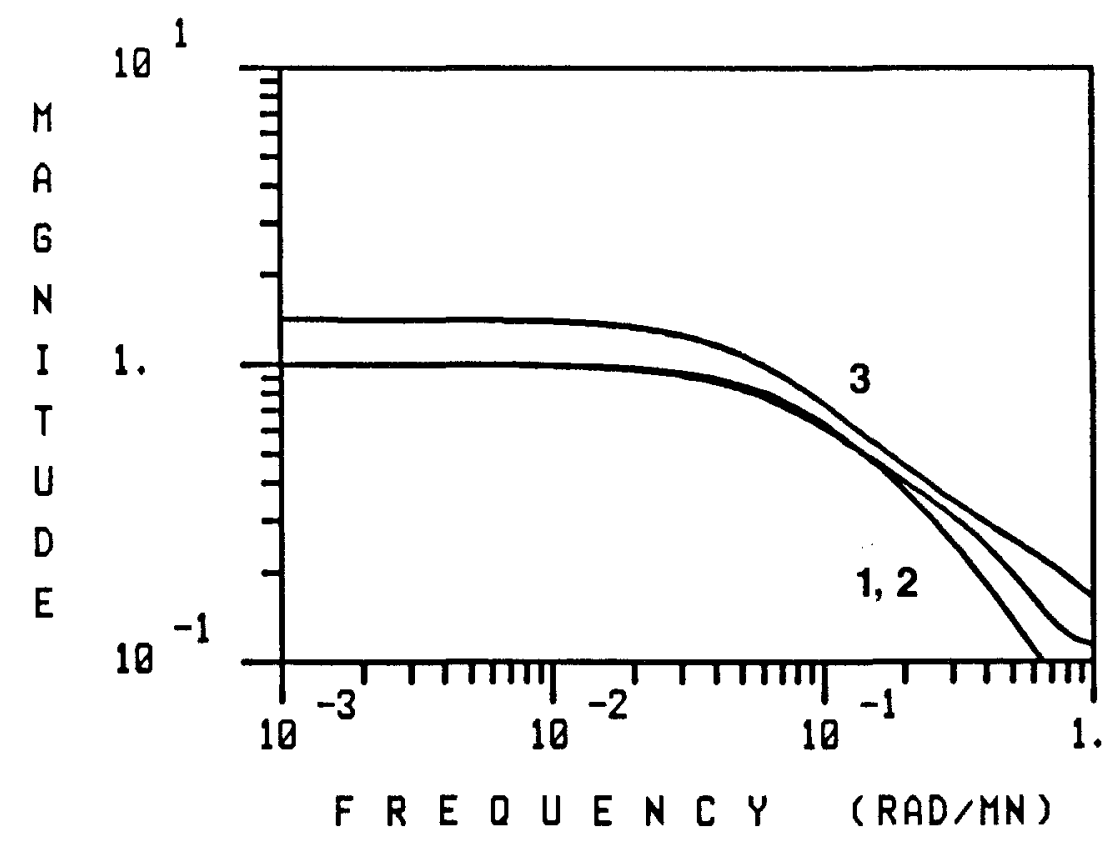

Figure 18. (Example 3), Lines 1 and $2=\left|\tilde{h}_{j}(j \omega)\right|, i=1,2$ respectively; Line $3=\mu^{-1}(E(j \omega))$, for diagonal controller with of $f$-diagonal pairings. 


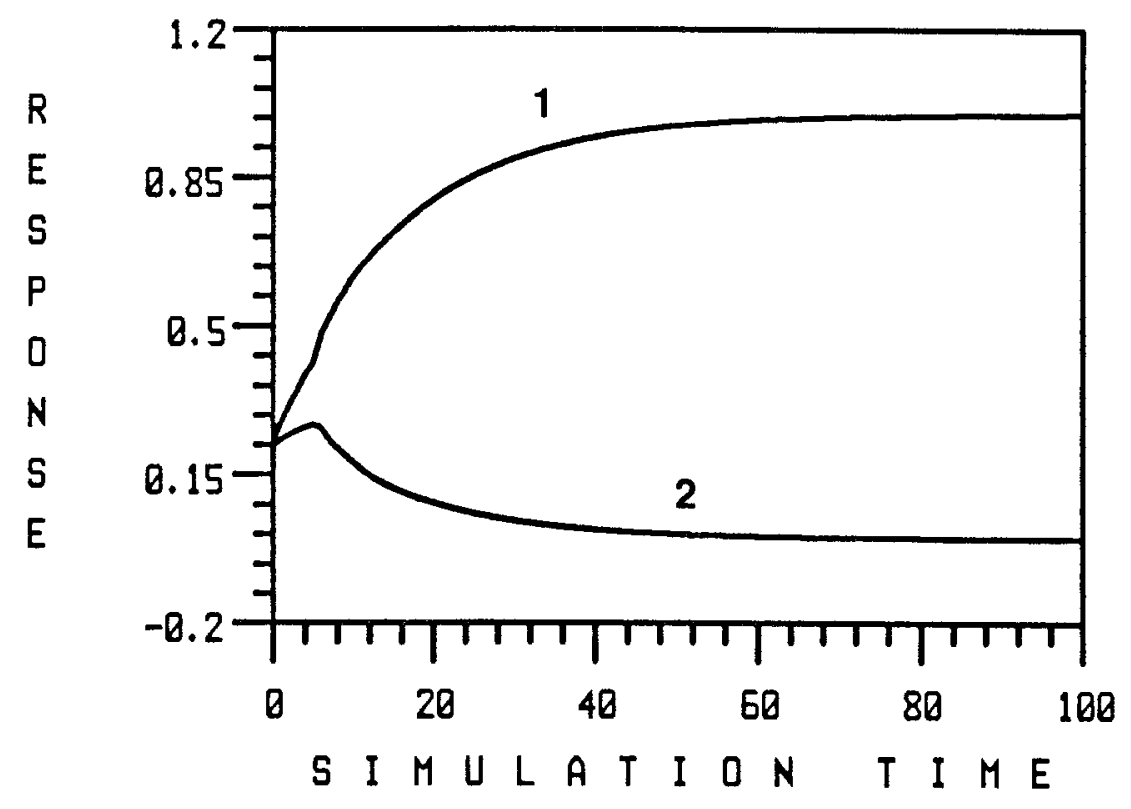

Figure 19. (Example 3), closed-10op response for unit step change in $r_{1}(s)$, off-diagonal pairings. 
CHAPTER IV: INTERACTION MEASURES FOR SYSTEMS UNDER DECENTRALIZED CONTROL 


\section{INTRODUCTION}

Let $G(s)$ be an nxn rational transfer function matrix relating the vector of system inputs $u$ to the vector of system outputs $y$. Let $r$ be the vector of reference signals or setpoints for the closed loop system. Assume that $u, y$ and $r$ have been partitioned in the same manner $u=$ $\left(u_{1}, u_{2}, \ldots u_{m}\right)^{\mathrm{T}}, \mathrm{y}=\left(\mathrm{y}_{1}, \mathrm{y}_{2}, \ldots \mathrm{y}_{\mathrm{m}}\right)^{\mathrm{T}}, r=\left(r_{1}, r_{2}, \ldots r_{\mathrm{m}}\right)^{\mathrm{T}}$. In this paper we mean by decentralized control that the controller $\mathrm{C}(\mathrm{s})$ is block diagonal (Fig. 1)

$$
u_{i}=c_{i}(s)\left(y_{i}-r_{i}\right)
$$

These constraints on the controller structure invariably lead to a performance deterioration when compared to the system with a full controller matrix. This sacrifice has to be weighed against the following two factors:

1. Hardware Simplicity: If $u_{i}, y_{i}$ are physically close but $u_{i}, y_{j}(i \neq j)$ are far apart, a full controller could require expensive communication links. Also, the controller hardware costs could be high if an implementation through analog circuitry is required. The purpose of an "interaction measure" would be to measure the performance degradation caused by the block diagonal controller. These considerations are relevant, for example, for large networks of power stations where the distances between the stations can be significant. Hardware issues are generally irrelevant in the context of process control: In all modern plants all measurements signals are sent into a central control room from where all the actuator signals originate.

2. Design Simplicity: If the block $G_{i j}(s)=0(i \neq j)$ then each controller $\mathrm{C}_{\dot{1}}(\mathrm{~s})$ can be designed for the isolated subsystem $\mathrm{G}_{\mathrm{i}}(\mathrm{s})$ 
without any loss of performance. If $G_{i j}(s)(i \neq j)$ is "small" then it should still be possible to design the controller for the essentially independent subsystems $G_{i i}(s)$. The advantage is that many fewer controller parameters need to be chosen than for the full system. This is particularly relevant in process control where often thousands of variables have to be controlled which could lead to an enormously complex controller. Here the objective of an "interaction measure" is more difficult to pinpoint. On one hand it should express the tuning difficulties caused by the offdiagonal system blocks; on the other it should give an indication of the performance degradation caused by the decentralized, i.e.,block diagonal controller. This problem is the subject of this paper. For our further developments we will make a more precise definition of "interaction measure" (IM) with reference to Fig. 2:

A controller

$$
C(s)=\operatorname{diag}\left(C_{1}(s), C_{2}(s), \ldots C_{m}(s)\right)
$$

is to be designed for the system

$$
\tilde{\mathrm{G}}(\mathrm{s})=\operatorname{diag}\left(\mathrm{G}_{11}(\mathrm{~s}), \mathrm{G}_{22}(\mathrm{~s}), \ldots \mathrm{G}_{\mathrm{mm}}(\mathrm{s})\right)
$$

such that the block diagonal closed loop system with the transfer matrix

$$
\tilde{\mathrm{H}}(\mathrm{s})=\tilde{\mathbf{G}}(\mathrm{s}) \mathbf{C}(\mathrm{s})(\mathrm{I}+\tilde{\mathrm{G}}(\mathrm{s}) \mathbf{C}(\mathrm{s}))^{-1}
$$

is stable ( $\delta=0$ in Fig. 2). An IM expresses the constraints imposed on the choice of the closed loop transfer matrix $\tilde{\mathrm{H}}(\mathrm{s})$ for the block diagonal system which guarantee that the full closed loop system

$$
H(s)=G(s) C(s)(I+G(s) C(s))^{-1}
$$

is stable (i.e., $\delta=1$ in Fig. 2).

As we will show in this paper, all popular IM's (Relative Gain, Rijnsdorp's IM, Nyquist Array) are either explicitly or implicitly based 
on this definition and thus have a rigorous theoretical basis. Other IMS (Witcher and McAvoy, 1977; Tung and Edgar, 1981; Gagnepain and Seborg, 1982) cannot be placed in this context which might be the reason that they have not been widely accepted.

Nevertheless this definition of an IM has its limitations and therefore the results should be interpreted with caution. The reason is that the IM is based on the block diagonal $\tilde{H}(s)$ which might or might not be indicative of the actual full closed loop transfer matrix $\mathrm{H}(\mathrm{s})$. Though the definition of the IM guarantees $H(s)$ to be stable it can be very badly behaved. The IM might indicate "small" interactions but the performance could be arbitrarily poor. This problem is the subject of current research.

The following developments will show that the matrix $(G(s)-\tilde{G}(s)) \tilde{G}^{-1}(s)$ plays a central role in interaction analysis. For simplicity of notation, we derine the matrices

$$
E(s)=(G(s)-\tilde{G}(s)) \tilde{G}^{-1}(s)
$$

and

$$
E^{\prime}(s)=\tilde{G}^{-1}(s)(\mathbf{G}(s)-\tilde{G}(s)) \text {. }
$$

Both $\mathbf{E}(\mathrm{s})$ and $\mathrm{E}^{\prime}(\mathrm{s})$ can be viewed as "relative errors" arising from the "approximation" of the full system $\mathbf{G}(\mathrm{s})$ by the block diagonal system $\tilde{\mathbf{G}}(\mathrm{s})$.

\section{STABILITY CONDITIONS}

Let us denote by $N(k, g(s))$ the net number of clockwise encirclements of the point $(k, 0)$ by the image of the Nyquist $D$ contour under $g(s)$. An application of the multivariable Nyquist criterion (Postlethwaite \& MacFarlane, 1979) to the control system in Fig. 1 
yields the following stability conditions.

Theorem 1: Assume that $\mathbf{G}(s)$ and $\tilde{G}(s)$ have the same number of RHP poles and that $\tilde{\mathrm{H}}(\mathrm{s})$ is stable. Then the closed loop system $\mathrm{H}(\mathrm{s})$ is stable if and only if

$$
\mathrm{N}(\mathrm{O}, \operatorname{det}(\mathrm{I}+\mathrm{E}(\mathrm{s}) \tilde{\mathrm{H}}(\mathrm{s})))=0
$$

Proof: The return difference operator for the full system $\mathrm{H}(\mathrm{s})$ can be factored as

$$
(I+G(s) C(s))=(I+E(s) \tilde{H}(s))(I+\tilde{G}(s) C(s))
$$

Let the number of open loop unstable poles of $G(s)$ and $\tilde{G}(s)$ be $p_{0} . H(s)$ is stable if and only if

$$
\mathrm{N}\left(0, \operatorname{det}(I+G(s) C(s))=N(0, \operatorname{det}(I+\tilde{G}(s) C(s)))+N\left(0, \operatorname{det}(I+E(s) \tilde{H}(s))=-p_{0}(10)\right.\right.
$$

Because $\tilde{\mathbf{H}}(\mathbf{s})$ is stable by assumption

$$
\mathrm{N}\left(0, \operatorname{det}(\mathrm{I}+\tilde{\mathrm{G}}(\mathrm{s}) \mathrm{C}(\mathrm{s}))=-\mathrm{p}_{0}\right.
$$

Substituting (11) into (10), (8) follows immediately. QED

Theorem 1 forms the cornerstone of much of the further development and deserves some discussion. First note that the poles of $G(s)$ are always a subset of the poles of $\tilde{\mathrm{G}}(\mathrm{s})$. Therefore the assumption of Theorem 1 that the number of unstable poles of $\mathbf{G}(s)$ and $\tilde{\mathbf{G}}(\mathrm{s})$ be the same is equivalent to the assumption that the location and multiplicity of the RHP poles be the same. Generically the subset is proper, and thus the assumption is generically never satisfied except trivially for stable systems. An exception are systems with integrators: Woolverton (1980) uses the model of a liquid-liquid separator (Table 1) which has one unstable pole at the origin. The diagonal system $\tilde{\mathbf{G}}(\mathrm{s})$ has the same unstable pole. Thus for all practical purposes the usefulness of Theorem 1 is limited to stable systems or systems with integrators which 
141

appear with a special structure in the model. With regard to the general problem of decentralized stabilization of large-scale systems we conclude that it is generically impossible to combine individually stabilized subsystems into an overall stable system.

The rearrangement of the block diagram in Fig. 2 for $\delta=1$ into the entirely equivalent form shown in Fig. 3 allows an interesting interpretation: (8) is a "robustness" condition for $\tilde{\mathrm{H}}(\mathrm{s})$ to remain stable under the multiplicative perturbation $\mathbf{E}(\mathrm{s})=(\mathrm{G}(\mathrm{s})-\tilde{\mathrm{G}}(\mathrm{s})) \tilde{\mathrm{G}}^{-1}(\mathrm{~s})$. Ideally one would want to select $\tilde{\mathrm{H}}(\mathrm{s})=\mathrm{I}$, i.e. perfect control. If $\mathrm{G}(\mathrm{s})=\tilde{\mathrm{G}}(\mathrm{s})$, i.e.,G(s) itself is block diagonal, then (8) is trivially satisfied. Thus the closed loop system $\mathrm{H}(\mathrm{s})$ is stable regardless of how $\widetilde{\mathrm{H}}(\mathrm{s})$ is chosen. If $\mathrm{G}(\mathrm{s}) \neq \tilde{\mathrm{G}}(\mathrm{s}), \tilde{\mathrm{H}}(\mathrm{s})$ has to be chosen such that (8) remains satisfied. Qualitatively, at least, it is clear that when $\mathbf{E}(\mathrm{s})$ is "large" $\tilde{\mathbf{H}}(\mathrm{s})$ has to be made "small" to avoid encirclements. A small $\tilde{\mathrm{H}}(\mathrm{s})$ implies poor performance. All IM's provide a quantitative indication of the constraints on $\tilde{\mathrm{H}}(\mathrm{s})$ imposed by $\mathbf{E}(\mathrm{s})$. As an illustration consider the following Corollary.

Corollary 1.1: Assume that

i) $\quad \mathrm{G}(\mathrm{s})$ and $\tilde{\mathrm{G}}(\mathrm{s})$ have the same RHP poles

ii) $\quad G(s) C(s)$ is strictly proper

iii) $\quad \tilde{\mathbf{H}}(\mathrm{s})$ is stable

iv) $\tilde{\mathrm{H}}(\mathrm{s})$ has vanishing tracking error for asymptotically constant inputs, i.e., $\tilde{\mathrm{H}}(0)=\mathrm{I}$.

then the closed loop system $\mathbf{H}(\mathrm{s})$ will be unstable if

$$
\lim _{s \rightarrow 0} \operatorname{det}\left(\mathbf{G}(s) \tilde{G}^{-i}(s)\right)<0
$$

Proof: Assumption ii) guarantees that

$$
\lim _{S \rightarrow \infty} \operatorname{det}(I+E(S) \tilde{H}(S))=1
$$


By assumption iv)

$$
\lim _{S \rightarrow 0} \operatorname{det}(I+E(s) \tilde{H}(s))=\lim _{s \rightarrow 0} \operatorname{det}\left(G(s) \tilde{G}^{-1}(s)\right)
$$

If (14) is negative (8) has to be violated because the Nyquist contour starts left and ends right of the origin. QED

If $\mathbf{G}(s)=\tilde{G}(s)$ then $\operatorname{det}\left(\mathbf{G}(s) \tilde{G}^{-1}(s)\right)=1$. If $\mathbf{G}(s)$ and $\tilde{\mathbf{G}}(s)$ are "very different", i.e., if the off diagonal terms are "large" it is likely that (12) holds. Then $\tilde{\mathrm{H}}(0)=\mathrm{I}$ will lead to an unstable system $\mathrm{H}(\mathrm{s})$. In order to obtain a stable system $\mathrm{H}(\mathrm{s})$, constraints have to be imposed on $\tilde{\mathrm{H}}(0)$, i.e., $\tilde{\mathrm{H}}(0) \neq \mathrm{I}$ and low frequency performance has to be sacrificed. Note that Corollary 1.1 is a generalization of Niederlinski's theorem and condition (Grosdidier et al., 1985)

$$
\frac{\operatorname{det}(\mathrm{G}(0))}{n}<0
$$

to block diagonal controllers and open-loop unstable systems. Its main limitation is that it is only necessary for stability. A simple sufficient condition is stated next.

Theorem 2: Assume that $\mathrm{G}(\mathrm{s})$ and $\tilde{\mathrm{G}}(\mathrm{s})$ have the same RHP poles and that $\tilde{\mathbf{H}}(\mathrm{s})$ is stable. Then the closed loop system $\mathbf{H}(\mathrm{s})$ is stable if

$$
\rho(E(j \omega) \tilde{H}(j \omega))<1 \quad \forall \omega
$$

where $\rho(\mathbf{A})$ is the spectral radius of $\mathbf{A}$.

Proof: (8) is satisfied if the characteristic loci (eigenvalues) of $\mathbf{E}(j \omega) \tilde{\mathbf{H}}(j \omega)$ do not encircle $(-1,0)$. (16) guarantees that this is the case. QED

Several approaches can be taken to derive explicit bounds on $\mathbb{H}(\mathrm{s})$ from (16). There is a trade-off between the assumptions made about the 
structure of $\tilde{\mathbf{H}}(\mathrm{s})$ and the "restrictiveness" of the bounds derived from (16). Less restrictive bounds on the magnitude of $\tilde{\mathbf{H}}(\mathbf{s})$ are obtained as more restrictive assumptions are made about the structure of $\tilde{H}(s)$. Assuming a highly structured form for $\tilde{\mathbf{H}}(\mathrm{s})$, i.e., $\tilde{\mathrm{H}}(\mathrm{s})=\tilde{\mathrm{h}}(\mathrm{s}) \cdot \mathrm{I}$, leads to the following bound.

Corollary 2.1: Assume $\tilde{\mathrm{H}}(\mathrm{s})=\tilde{\mathrm{h}}(\mathrm{s}) \cdot \mathrm{I}$. Under the assumptions of Theorem 2 the closed loop system $\mathrm{H}(\mathrm{s})$ is stable if

$$
|\tilde{h}(j \omega)|<\rho^{-1}(E(j \omega)) \quad \forall \omega
$$

The form of $\tilde{\mathbf{H}}(\mathrm{s})$ assumed for Corollary 2.1 is very restrictive but $\rho^{-1}(E(j \omega))$ is the least restrictive magnitude bound that can be derived for $\tilde{\mathbf{H}}(\mathrm{s})$ from (16). It is more practical to use the fact that the spectral radius of a matrix is bounded above by any induced norm. Corollary 2.2: Under the assumptions of Theorem 2 the closed 1oop system $H(s)$ is stable if

$$
|| E(j \omega) \tilde{\mathrm{H}}(j \omega)||<1 \quad \forall \omega
$$

where || $\mathbf{A}||$ denotes any induced norm of $\mathbf{A}$.

As we will show in the next sections Corollary 2.2 is the basis for all the "diagonal dominance" results. Bounds on ||$\tilde{\mathrm{H}}(j \omega)||$ can also be obtained directly from (18)

$$
|| \tilde{H}(j \omega)||<|| E(j \omega)||^{-1} \quad \forall \omega
$$

(19) makes no assumptions about the structure of $\tilde{\mathbf{H}}(\mathrm{s}) . \tilde{\mathbf{H}}(\mathrm{s})$ could actually be a full matrix, which is not meaningful in the present context and clearly indicates that the bound (19) is conservative. The conservativeness also depends on the type of norm used. It is therefore natural to look for an expression like (19) which takes into account the structure of $\tilde{\mathbf{H}}(\mathrm{s})$ and represents an optimal bound in the following sense: Let $\tilde{\mathbf{H}}(\mathrm{s})$ be block diagonal and norm bounded 


$$
\begin{gathered}
144 \\
\tilde{\mathrm{H}}(s)=\operatorname{diag}\left(\tilde{\mathrm{H}}_{i}(s), \ldots . \tilde{\mathrm{H}}_{\mathrm{m}}(s)\right) \\
\sigma_{\max }\left(\tilde{\mathrm{H}}_{i}(j \omega)\right)<\delta(\omega) \quad \forall i, \omega
\end{gathered}
$$

or equivalently

$$
\sigma_{\max }(\tilde{\mathbf{H}}(j \omega))<\delta(\omega) \quad \forall \omega
$$

where $\sigma_{\max }(\mathbf{A})$ is the maximum singular value of $\mathbf{A}$. A real positive

function $\mu(E(j \omega))$ is desired with the property that (8) is satisfied for all matrices $\tilde{\mathrm{H}}(\mathrm{j} \omega)$ satisfying (20) and (21) if and only if

$$
\sigma_{\max }(\tilde{\mathrm{H}}(j \omega))<\mu^{-1}(\mathrm{E}(j \omega)) \quad \forall \omega
$$

$\mu$ turns out to be the Structured Singular Value (SSV) suggested by Doyle (1982) for the analysis of feedback systems with structured uncertainties.

Theorem 3: Assume that $\mathbf{G}(s)$ and $\tilde{\mathbf{G}}(\mathrm{s})$ have the same RHP poles and that $\tilde{\mathbf{H}}(\mathrm{s})$ is stable. Then the closed loop system $\mathrm{H}(\mathrm{s})$ is stable if

$$
\sigma_{\max }(\tilde{\mathrm{H}}(j \omega))<\mu^{-1}(E(j \omega)) \quad \forall \omega
$$

(23) is the tightest norm bound in the sense that if there is a system $\tilde{\mathrm{H}}_{1}(\mathrm{~S})$ which violates (23)

$$
\sigma_{\max }\left(\tilde{\mathrm{H}}_{i}(j \omega)\right)>\mu^{-1}(E(j \omega))
$$

then there exists another system $\tilde{\mathrm{H}}_{2}(\mathrm{~s})$ such that

$$
\sigma_{\max }\left(\tilde{\mathrm{H}}_{1}(j \omega)\right)=\sigma_{\max }\left(\tilde{\mathrm{H}}_{2}(j \omega)\right)
$$

for which (8) is violated and $\mathbf{H}(\mathrm{s})$ is unstable.

\section{INTERACTION MEASURES}

1. Diagonal Dominance Criteria

Assume that $\tilde{\mathrm{G}}(\mathrm{s})$ and $\tilde{\mathrm{H}}(\mathrm{s})$ are diagonal and that the 1 -norm is used in (18). Then (18) becomes 


$$
\sum_{i \neq j}\left|\frac{g_{i j}(j \omega)}{g_{j j}(j \omega)} \tilde{h}_{j}(j \omega)\right|<1 \quad \forall j, \omega
$$

Or

$$
\left|\tilde{h}_{j}(j \omega)\right|<\left[\sum_{i \neq j}\left|\frac{g_{i j}(j \omega)}{g_{j j}(j \omega)}\right|\right]^{-1} \quad \forall_{j}, \omega
$$

(27) is the IMC interaction measure for column dominance (Economou and Morari, 1985). It expresses the constraints the individual loops $\tilde{h}_{j}(s)$ must satisfy for the overall system to be stable. A plot of the RHS of (27) as a function of frequency is a good indicator of the bandwidth over Which good control can be achieved. Clearly, if all the of $f$ diagonal elements in column $j$ are zero, $\tilde{h}_{j}(s)$ is unconstrained. (26) can also be written as

$$
\sum_{i \neq j}\left|g_{i j}(j \omega)\right|<\left|g_{j j}(j \omega)+\frac{1}{c_{j}(j \omega)}\right| \quad \forall j, \omega
$$

When $c_{j}(s)$ is a constant, the LHS of (28) defines the radii of the circles forming the Gershgorin bands for column dominance (Rosenbrock, 1974). The role of the "relative error" $\mathbf{E}(\mathrm{s})$ becomes apparent in the definition of dominance. Definition 1: Let $\tilde{G}(j \omega)$ be diagonal. The complex matrix $\mathbf{G}(j \omega)$ is said to be column dominant if

$$
|| E(j \omega)||<1
$$

or

$$
\sum_{i \neq j}\left|g_{i j}(j \omega)\right|<\left|g_{j j}(j \omega)\right| \quad \forall j
$$

When $\mathbf{G}(j \omega)$ is diagonally dominant for all $\omega$, a fortunate and rare situation, then the constraint (27) on $\left|\tilde{h}_{j}(j \omega)\right|$ is very mild 


$$
\left|\tilde{h}_{j}(j \omega)\right|\langle\alpha \text { where } \alpha>1 \quad \forall j, \omega
$$

and there are no limitations on the bandwidth imposed by the interactions.

We remark that

$$
\operatorname{det}\left(I+(G(s)-\tilde{G}(s)) \tilde{G}^{-1}(s) \tilde{H}(s)\right)=\operatorname{det}\left(I+\tilde{G}^{-1}(s) \tilde{H}(s)(G(s)-\tilde{G}(s))\right)
$$

Therefore another sufficient stability condition which is similar to (18) is

$$
|| \tilde{G}^{-1}(j \omega) \tilde{H}(j \omega)(G(j \omega)-\tilde{G}(j \omega))||<1 \quad \forall \omega
$$

When the $\infty$-norm is used in (31) the IMC interaction measure and the circle radii for row dominance are obtained in a straightforward fashion. 2. Generalized Diagonal Dominance Criteria

Let us assume again that $\tilde{\mathrm{G}}(\mathrm{s})$ and $\tilde{\mathrm{H}}(\mathrm{s})$ are diagonal. If the inputs of $\mathrm{G}(\mathrm{s})$ are scaled by a diagonal nonsingular matrix $\mathrm{S}_{2}$ and the outputs by a diagonal nonsingular matrix $\mathbf{S}_{\downarrow}$ and if the controller $\mathbf{C}(\mathrm{s})$ is scaled accordingly the stability of the system should be unaffected. This can be seen easily from (8)

$$
\begin{gathered}
\operatorname{det}\left(I+\left(S_{1} G(s) S_{2}-S_{1} \tilde{G}(s) S_{2}\right) S_{2}^{-1} \tilde{G}^{-1}(s) S_{1}^{-1} \cdot S_{1} \tilde{H}(s) S_{1}^{-1}\right)= \\
\operatorname{det}\left(I+S_{1}(G(s)-\tilde{G}(s)) \tilde{G}^{-1}(s) \tilde{H}(s) S_{1}^{-1}\right)= \\
\operatorname{det}\left(I+S_{1}(G(s)-\tilde{G}(s)) \tilde{G}^{-1}(s) S_{1}^{-1} \tilde{H}(s)\right)= \\
\operatorname{det}(I+E(s) \tilde{H}(s))
\end{gathered}
$$

A similar development holds for the right hand side of (30). Though stability is independent of scaling the sufficient stability condition (19) is not. It is natural to seek the scaling which makes (19) least conservative. Equation (32c) shows that for the error matrix $E(s)$ only one scaling matrix $\left(S_{1}\right)$ is necessary, the other one cancels. Thus we are seeking to solve the minimization problems

$$
\min _{S_{1}}|| S_{1} E(j \omega) S_{1}^{-1} \|_{1}
$$


and

$$
\min _{\mathrm{S}_{2}}|| \mathrm{S}_{2}^{-1} \mathrm{E}^{\prime}(j \omega) \mathrm{S}_{2}||_{\infty}
$$

The solutions of ( $33 \mathrm{a}$ and $\mathrm{b}$ ) are provided by the Perron-Frobenius Theorem (Seneta, 1973; Mees, 1981; Limebeer, 1982).

Theorem 4:

$$
\min _{S_{1}}|| S_{1} E(j \omega) S_{1}^{-1}||_{1}=\min _{S_{2}}|| S_{2}^{-1} E^{\prime}(j \omega) S_{2}||_{\infty}=\rho(|E(j \omega)|)
$$

where $|\mathbf{A}|$ denotes the matrix $\mathbf{A}$ with all its elements replaced by their magnitudes.

Corollary 2.3: Assume that $\mathrm{G}(\mathrm{s})$ and $\tilde{\mathrm{G}}(\mathrm{s})$ have the same RHP poles and that $\tilde{H}(s)$ is stable. Then the closed loop system $H(s)$ is stable if

$$
\left|\tilde{h}_{j}(j \omega)\right|<\rho^{-1}(|E(j \omega)|) \quad \forall_{j}, \omega
$$

Note the similarity between Corollary 2.1 and 2.3. (17) is a tighter bound than (35) because the spectral radius bounds any norm - also when it is minimized - from below. However, for obtaining this tighter bound $\tilde{\mathrm{H}}(\mathrm{s})$ had to be restricted to $\tilde{\mathrm{H}}(\mathrm{s})=\tilde{\mathrm{h}}(\mathrm{s}) \cdot \mathrm{I}$.

(35) can be written as

$$
g_{j j}(j \omega) \rho(|E(j \omega)|)<\left|g_{j j}(j \omega)+\frac{1}{c_{j}(j \omega)}\right| \quad \forall j, \omega
$$

When $c_{j}(j \omega)$ is a constant the LHS of (36) defines the radii of the circles for generalized diagonal dominance (Mees, 1981; Limebeer, 1982). The "relative error" $\mathbf{E}(\mathrm{s})$ appears again explicitely in the definition of generalized diagonal dominance.

Definition 2: Assume $\tilde{\mathbf{G}}(j \omega)$ to be diagonal. The complex matrix $\mathbf{G}(j \omega)$ is said to be generalized diagonal dominant if

$$
\rho(|E(j \omega)|)<1
$$

(37) is clearly less conservative than (29a) because it is the result of a minimization. On the other hand it would be incorrect to view (35) or 
(36) as less conservative than (27) or (28). (27) provides individual bounds for each of the single loop transfer functions $\tilde{h}_{j}(s)$ and thus allows trade-offs between the different loops. The optimization giving rise to (35) minimizes the worst bound and in the process makes all the bounds even. However (35) has the advantage that independent of the number of system inputs and outputs the design engineer can determine from a single curve if the selected control structure leads to significant performance deterioration or not. Even the trade-off problem can be addressed in (35) by introducing weighting matrices as we will explain later.

Both the diagonal dominance and generalized diagonal dominance concept can be extended to block diagonal systems (Feingold and Varga, 1962; Limebeer, 1982). The bounds on ||$\tilde{\mathrm{H}}_{1}(j \omega)||$ obtained by this approach are excessively conservative and therefore not very useful in most practical applications.

\section{The SSV-Interaction Measure}

Theorem 3 states that for stability the magnitude of the diagonal blocks $\tilde{\mathrm{H}}_{i}(\mathrm{~s})$ has to be constrained by the reciprocal of the SSV $\mu$ of the "relative error" matrix

$$
\sigma_{\max }\left(\tilde{H}_{i}(j \omega)\right)<\mu^{-1}(E(j \omega)) \quad \forall i, \omega
$$

The value of $\mu$ depends on the structure assumed for $\tilde{\mathrm{H}}(\mathrm{s})$. Its computation is an active area of research at present. Two optimization problems are suggested by the bounds derived by Doyle (1982)

$$
\max _{\mathbf{U} \in \mathcal{V}} \rho(\mathrm{UE}(j \omega))=\mu(\mathrm{E}(j \omega)) \leq \inf _{\mathbf{D} \varepsilon \mathscr{Q}} \sigma_{\max }\left(\mathrm{DE}(j \omega) \mathrm{D}^{-1}\right)
$$

where $U$ is the set of all unitary matrices with the same block diagonal structure as $\tilde{\mathrm{H}}(\mathrm{s})$ 
149

$$
\mathcal{U}=\left\{\operatorname{diag}\left\{\mathbf{U}_{1}, \ldots \mathrm{U}_{\mathrm{m}}\right\}, \mathrm{U}_{\mathrm{i}} \text { unitary }\right\}
$$

and

$$
\mathscr{D}=\left\{\operatorname{diag}\left\{\mathrm{d}_{\perp} \mathrm{I}_{1}, \ldots \mathrm{d}_{\mathrm{m}} \mathrm{I}_{\mathrm{m}}\right\}, \mathrm{d}_{\mathrm{i}} \in \mathrm{R}_{+}\right\}
$$

The block structure of $\mathscr{D}$ is again defined by the block structure of $\tilde{\mathbf{H}}(\mathrm{s})$. Unfortunately the maximization in (39) is nonconvex. The optimization implied by the upper bound is convex but the infimum is only equal to $\mu$ for $m \leq 3$. Experience has shown the upper bound to be very good even when $\mathrm{m}>3$.

(39) implies that

$$
\rho(E(j \omega)) \leq \mu(E(j \omega))
$$

This confirms our finding of Corollary 2.1 that $\rho^{-1}(E(j \omega))$ constitutes the loosest bound but that it is only correct for the rather restricted structure $\tilde{H}(s)=\tilde{h}(s) \cdot I$. Equation (39) also implies that

$$
\mu(E(j \omega)) \leq \sigma_{\max }(E(j \omega))
$$

This is consistent with the conservative result (19) when the spectral norm is used and when no structural constraints are put on $\tilde{\mathrm{H}}(\mathrm{s})$. Not surprisingly $\mu(E(j \omega))$ lies between the extremes when $\tilde{H}(s)$ has no specific structure at all and when $\tilde{\mathrm{H}}(\mathrm{s})=\tilde{\mathrm{h}}(\mathrm{s}) \cdot \mathrm{I}$.

Note that $\mu$ treats both diagonal and block diagonal $\tilde{\mathrm{H}}(\mathrm{s})$ in a unified "optimal" manner. Just like in the case of generalized diagonal dominance all loops are given equal preference. We will show later how weighting functions can be used to establish a preference structure.

For diagonal $\tilde{\mathrm{H}}(\mathrm{s})$ (38) can be rewritten as

$$
g_{j j}(j \omega) \mu(E(j \omega))<\left(g_{j j}(j \omega)+\frac{1}{c_{j}(j \omega)}\right) \quad \forall_{j}, \omega
$$

The LHS of (44) defines the radii of circles which form the equivalent of Gershgorin bands on the Nyquist plot of $g_{j j}(s)$. The bands are the narrowest ones possible in the discussed sense. They are always 
narrower or equal to the bands from generalized diagonal dominance (36). 4. Interaction Measures for $2 \times 2$ Systems

The interaction measures for $2 \times 2$ systems which are most widely used industrially were proposed by Rijnsdorp (1965) and Bristol (1966). The Rijnsdorp Interaction Measure is defined as

$$
K(s)=\frac{g_{12}(s) g_{21}(s)}{g_{11}(s) g_{22}(s)}
$$

and the Relative Gain as

$$
\lambda=\lim _{S \rightarrow 0} \frac{1}{1-k(s)}
$$

Theorem 1 can be rephrased specifically for $2 x 2$ systems.

Corollary 1.2: Assume that $\mathbf{G}(s)$ and $\tilde{\mathbf{G}}(s)$ have the same RHP poles and that $\tilde{\mathbf{H}}(\mathrm{s})$ is stable. Then the closed loop system $\mathrm{H}(\mathrm{s})$ is stable if and only if

$$
N(+1, z(s))=0
$$

where

$$
z(s)=\tilde{h}_{1}(s) \tilde{h}_{2}(s) k(s)
$$

Corollary 1.2 shows that $k(s)$ is rigorously related to closed loop stability and that both the gain and the phase information of $k(s)$ can be utilized to establish the constraints imposed on $\tilde{h}_{i}(s),(i=1,2)$, by the interactions. The application of Cor. 1.2 will be discussed later in the paper.

From (39) it can be easily shown that for $2 \times 2$ systems

$$
\rho(|E(j \omega)|)=\rho(E(j \omega))=\mu(E(j \omega))=\sqrt{|\kappa(j \omega)|}
$$

Corollary 3.1: Assume that $\mathrm{G}(\mathrm{s})$ and $\tilde{\mathbf{G}}(\mathrm{s})$ have the same RHP poles and that $\tilde{\mathbf{H}}(\mathrm{s})$ is stable. Then the closed loop system $\mathrm{H}(\mathrm{s})$ is stable if

$$
\left|\tilde{h}_{\dot{1}}(j \omega)\right|<|k(j \omega)|^{-1 / 2}=\rho^{-1}\left(E(j(\omega))=\mu^{-1}(E(j \omega)) \quad \forall i, \omega\right.
$$


Corollary 3.1 is more conservative than Cor. 1.2 because the phase information is discarded but it is much easier to apply. Corollary 3.1 establishes the Rijnsdorp Interaction Measure as optimal for $2 \times 2$ systems in the sense of the SSV $\mu$.

McAvoy (1981) has argued that a frequency dependent definition (46) of the Relative Gain $\lambda$

$$
\lambda(s)=\frac{1}{1-K(s)}
$$

could be used in a semiquantitative manner for loop tuning. If $|\kappa(j \omega)|>$ 1 then (51) satisfies

$$
|\lambda(j \omega)|<\frac{1}{|\kappa(j \omega)|-1}
$$

and for $|k(j \omega)| \gg 1$ we have the approximation

$$
|\lambda(j \omega)|<|k(j \omega)|^{-1 / 2}
$$

Thus $|\lambda(j \omega)|$ could be used instead of $|\kappa(j \omega)|^{-1 / 2}$ in Cor. 3.1. However, this approximation holds only for $|\kappa(j \omega)| \gg 1$, i.e. when the interactions are strong and is unnecessarily conservative. Therefore the "dynamic extension" of the Relative Gain is not recommended as an Interaction Measure.

From (46), (49) and Definition 2 we derive the following fact:

Fact 1: The following are equivalent for $2 \times 2$ systems
(a) $\mathbf{G}(0)$ is generalized diagonally dominant
(b) $\rho(E(0))<1$
(c) $\quad \rho(|E(0)|)<1$
(d) $\mu(E(0))<1$
(e) $|\kappa(0)|<1$
(f) $\lambda>\frac{1}{2}$

This explains the famous rule of thumb (Shinskey, 1979) for connecting 
input-output pairs with $\lambda>\frac{1}{2}$ as a simple generalized diagonal dominance criterion. Dominance at $\omega=0$ has important practical consequences. The following corollary follows directly from Theorem 3 .

Corollary 3.2: Assume that for a $2 \times 2$ system $\mathbf{G}(\mathrm{s})$ and $\tilde{\mathbf{G}}(\mathrm{s})$ have the same RHP poles, and that any of the conditions of Fact 1 are satisfied. Then there exists a diagonal controller with integral action, i.e., $\tilde{\mathrm{H}}(0)=I$, such that both $\tilde{\mathrm{H}}(\mathrm{s})$ and $\mathrm{H}(\mathrm{s})$ are stable.

From (49), Cor. 2.3 and Thm. 3 it is self evident that either $\rho^{2}(|\mathbf{E}(j \omega)|)$ or $\mu^{2}(\mathbf{E}(j \omega))$ is the most natural extension of the Rijnsdorp Interaction Measure $k(s)$ to systems larger than $2 x 2$. Other physically motivated extensions which have been proposed (Jensen et al.,1985) have no apparent theoretical justification. At present $\rho(|\mathbf{E}(j \omega)|)$ is simpler to compute than $\mu(E(j \omega))$ but it is less tight. By comparing $\rho(|E(j \omega)|)$ with $\rho(E(j \omega))$, the lower bound on $\mu(E(j \omega))$, a decision can be made on a case by case basis if the effort of computing $\mu(\mathbf{E}(j \omega))$ is worthwhile.

\section{WEIGHTING MATRICES}

The limitation associated with the result of Theorem 3 is that $\mu$ gives equal preference to all the loops. In some cases this may impose an early and perhaps unnecessary roll-off for some of the $\tilde{\mathbf{H}}_{\mathfrak{i}}(\mathrm{s})$. The introduction of a weighting matrix $\mathbf{W}(\mathrm{s})$, with block-diagonal structure equal to that of $\tilde{\mathrm{H}}(\mathrm{s})$, into the expression

$$
(\mathrm{I}+\mathrm{E}(\mathrm{s}) \tilde{\mathbf{H}}(\mathrm{s}))
$$

circumvents this problem. (54) becomes

$$
\left(\mathrm{I}+\mathrm{E}(\mathrm{s}) \mathrm{W}(\mathrm{s}) \mathrm{W}^{-1}(\mathrm{~s}) \mathbf{H}(\mathrm{s})\right)
$$

and the sufficient stability condition of Theorem 3 (cf. (23)) becomes

$$
\sigma_{\max }\left(W^{-1}(j \omega) \tilde{H}(j \omega)\right)<\mu^{-1}(E(j \omega) W(j \omega)) \quad \forall \bar{\omega}
$$


153

In practice, a proper weighting matrix will express a performance

constraint imposed on one or more of the $\tilde{\mathbf{H}}_{\dot{1}}(\mathrm{~s})$ and due, for example, to constraints in manipulated variables or plant uncertainty. This procedure will later be illustrated in an example.

\section{EXAMPLES}

Example 1. We consider the distillation column of Doukas and Luyben (1978). The transfer function matrix for a $3 \times 3$ subsystem is shown in Table 2.

Line 1 in Fig. 4 shows a plot of $\mu^{-1}(E(j \omega))$ for the fullydecentralized control system with (diagonal) pairings $((1,1),(2,2)$, $(3,3))$. This curve shows that a fully-decentralized controller with integral action cannot be designed on the basis of Theorem 3 since $\mu^{-1}(\mathbf{E}(0))$ < 1. This constraint can be relaxed by considering a more complex controller structure. Line 2 in the same figure shows a plot of $\mu^{-1}(E(j \omega))$ for the block-decentralized control system with pairings $((1-2,1-2),(3,3))$. In this case, a controller with integral action is possible since $\mu^{-1}(E(0))>1$. However, the interactions limit the achievable closed loop bandwidth to about $0.2 \mathrm{rad} / \mathrm{min}$.

$\rho^{-1}(|E(j \omega)|)$ for the block-decentralized controller is shown as Line 3 in Fig. 4. A comparison of Lines 2 and 3 demonstrates the conservativeness associated with $\rho^{-1}(|\mathbf{E}(j \omega)|)$ as IM.

Example 2. Block-decentralized controllers do not necessarily yield values of $\mu$ less conservative than fully-decentralized controllers. For the system in Table 2, consider a fully-decentralized control structure implemented on the variable pairs $((1,2),(2,1),(3,3))$ and a blockdecentralized control structure implemented on the pairings $((1,2),(2-3$, 
1-3)). Lines 1 and 2 in Fig. 5 show plots of $\mu^{-1}(E(j \omega))$ for the fullyand block-decentralized control structures, respectively. On the basis of Theorem 3, a controller with integral action is only possible with the fully-decentralized control structure.

Example 3. We assume now a fully-decentralized control structure for the system in Table 2 implemented on the variable pairs $((1,2),(2,1)$, $(3,3))$. The objective is to demonstrate the response of the closed-1oop system when the three controllers are designed on basis of Theorem 3 . Figure 6 shows a plot of $\mu^{-1}(E(j \omega)$ ) (Line 1). The interactions limit the closed loop bandwidth to about $0.08 \mathrm{rad} / \mathrm{min}$. Figure 6 also shows plots of $\left|\tilde{h}_{i}(j \omega)\right|$, $(i=1,3)$, for three different sets of controllers. In the first case, the three controllers are chosen such that

$$
\tilde{h}_{i}(s)=\frac{e^{-\tau_{i} s}}{(4 s+1)^{3}} \quad i=1,3
$$

while in the second and third cases the controllers are chosen such that

$$
\tilde{h}_{i}(s)=\frac{e^{-\tau_{i} s}}{(10 s+1)^{3}} \quad i=1,3
$$

and

$$
\tilde{h}_{\dot{i}}(s)=\frac{e^{-\tau_{i} s}}{(25 s+1)^{3}} \quad i=1,3
$$

respectively. Here, $\tau_{i},(i=1,3)$, are the time delays in $g_{12}(s), g_{21}(s)$ and $\mathrm{g}_{33}(\mathrm{~s})$, respectively. The responses of the closed-1oop systems for these three sets of controllers were tested for step changes in $r_{2}(s)$. The responses corresponding to (57), (58) and (59) are shown in Figs. (7a), (7b) and (7c) respectively. It is apparent that as the $\tilde{h}_{i}(s)$ are moved away from the stability bound (Line 1) and thus made more conservative, the closed-loop responses become more sluggish but less oscillatory. Example 4. We consider the system of Example 3 with the weighting 
matrix

$$
W(s)=\operatorname{diag}\left[\frac{1}{(20 s+1)^{2}}, \frac{1}{(60 s+1)^{2}}, 1\right]
$$

This weighting matrix imposes roll-off frequencies of $0.05 \mathrm{rad} / \mathrm{min}$ and $0.017 \mathrm{rad} / \mathrm{min}$ for $\tilde{h}_{1}(\mathrm{~s})$ and $\tilde{h}_{2}(\mathrm{~s})$, respectively. On the other hand, the constraint on the other transfer function is relaxed as shown by the plot of $\mu^{-1}(\mathbf{E}(j \omega) \mathbf{W}(j \omega))$ in Fig. 8 (Line 1). A controller can therefore be designed giving $\tilde{h}_{3}(s)$ an arbitrarily large bandwidth with a guarantee that $H(s)$ will be stable. Line 2 in this figure is a plot of $\mu^{-1}(E(j \omega))$ and is shown only for comparison.

Example 5. We consider a $2 \times 2$ system with transfer function matrix

$$
G(s)=\left[\begin{array}{cc}
\frac{5}{(4 s+1)} & \frac{2.5 e^{-5 s}}{(2 s+1)(15 s+1)} \\
\frac{-4 e^{-6 s}}{(20 s+1)} & \frac{1}{(3 s+1)}
\end{array}\right]
$$

under decentralized control. For a decentralized controller with structure $((1,1),(2,2)), k^{-1}(0)=0.5$, whereas for structure $((1,2),(2,1))$ $K^{-1}(0)=2$. Fact 1 suggests that the decentralized controller should be implemented on this latter pairing in order to allow integral action on both control loops (Cor. 3.2). This choice, however, appears undesirable because of the time delays and relatively slow dymamics associated with $g_{12}(s)$ and $g_{21}(s)$. In order to investigate the feasibility and desirability of the pairing $((1,1),(2,2))$ the necessary and sufficient stability condition of Cor. 1.2 has to be utilized rather than the conservative sufficient condition of Cor. 3.1 and 3.2 .

We first consider a control structure based on the diagonal transfer functions $g_{11}(s)$ and $g_{22}(s)$. Figure 9 shows the Bode plot of $\kappa(s)$ for this structure (Line 1). Since encirclement of the point $(1,0)$ is easily avoided, it should be possible to design controllers giving rise 
to two closed-10op transfer functions $\tilde{h}_{1}(s)$ and $\tilde{h}_{2}(s)$ such that $z(s)=$ $\tilde{h}_{1}(s) \tilde{h}_{2}(s)_{k}(s)$ also avoids the encirclement. Choosing the two controllers such that

$$
\tilde{h}_{1}(s)=\tilde{h}_{2}(s)=\frac{1}{(s+1)^{2}}
$$

satisfies this constraint as shown in Fig. 9 (Line 2). Figure 10 shows the closed loop response of the system for a unit step change in $r_{1}(s)$. By comparison, Fig. 11 shows the closed-loop response of the system for the same forcing function but with the controller implemented on the offdiagonal transfer functions $\mathrm{g}_{12}(\mathrm{~s})$ and $\mathrm{g}_{21}(\mathrm{~s})$. The controllers for this response were tuned on basis of Theorem 3. The better response in Fig. 10 justifies the diagonal pairings.

This example shows that it is useful to consider both the magnitude and the phase of $\kappa(\mathrm{s})$ for variable pairing and controller design in $2 \times 2$ systems. It also shows that the performance of these systems can be quite acceptable when $\lambda<1 / 2$. In general, for $2 \times 2$ systems, variables should be paired such that $k(0)<1$ as a consequence of Fact 1 . However, if $k(0)>1$ but $k(s)$ is a decreasing function of frequency with a slope between -1 and -2 , this pairing may often be preferable as illustrated in Example 5.

\section{CONCLUSION}

By interpreting interactions in decentralized control systems as multiplicative uncertainty, we have given a unified treatment of all currently available measures of interactions. Results show that these measures express a bound on the magnitude of the decentralized closed loop transfer matrix $\tilde{\mathbf{H}}(\mathrm{s})$ imposed by the "relative error matrix" $\mathrm{E}(\mathrm{s})=$ 
$(\mathrm{G}(\mathrm{s})-\tilde{\mathrm{G}}(\mathrm{s})) \tilde{\mathrm{G}}^{-1}(\mathrm{~s})$, where $\tilde{\mathrm{G}}(\mathrm{s})=\operatorname{block}-\operatorname{diag}(\mathrm{G}(\mathrm{s}))$.

Using the notion of Structured Singular Value (SSV), we have defined a new dynamic interaction measure which provides the tightest possible bound which can be defined on $\sigma_{\max }(\tilde{\mathrm{H}}(\mathrm{s})) \div$ not on $\tilde{\mathrm{H}}(\mathrm{s})$. This measure, called the SSV-Interaction Measure, treats multivariable systems of arbitrary size under feedback with diagonal or block-diagonal controllers, all in a unified manner.

The SSV-Interaction Measure can be conveniently displayed on an amplitude-frequency diagram. Such plots can be used not only to predict the stability of decentralized control systems but also to predict the performance loss caused by these control structures. In particular, the steady-state value of the SSV-Interaction Measure provides a sufficient condition for achieving offset-free performance with the closed-1oop system. In the case of decentralized $2 \times 2$ systems, this measure is found to be closely related to Rijnsdorp's Interaction Measure.

\section{ACKNOWLEDGEMENTS}

Support from the Department of Energy and the National Science Foundation are gratefully acknowledged. We are thankful to J. Doyle and S. Skogestad for many useful discussions. 
REFERENCES

Bristol, E. H. (1966). On a New Measure of Interaction for Multivariable Process Control. EEE Trans. Autom. Control, AC-11, 133-134.

Doukas, N. and W. Luyben (1978). Control of Sidestream Columns Separating Ternary Mixtures. Anal. Instrum., 16, 51-58.

Doyle, J. (1982). Analysis of Feedback Systems with Structured Uncertainties. IEE Proc., 129, 242-250.

Economou, C. and M. Morari (1985). The IMC Interaction Measure. Ind. Eng. Chem. Process Des. Dev. (accepted for publication). Feingold, D. G. and R. S. Varga (1962). Block Diagonally Dominant Matrices and Generalizations of the Gerschgorin Circle Theorem. Pacific J. Maths., 12, 1241-1250.

Gagnepain, J. P. and D. E. Seborg (1982). Analysis of Process Interactions with Applications to Multiloop Control System Design. Ind. Eng. Chem. Process Des. Dev., 21, 5-11.

Grosdidier, P., M. Morari and B. R. Holt (1985). Closed-Loop Properties from Steady-State Gain Information. Ind. Eng. Chem. Fund., 24, $221-235$.

Jensen, N., D. G. Fisher and S. L. Shah (1985). Interaction Analysis in Multivariable Control System Design. AIChE J. (in press). Limebeer, D. J. N. (1982). The Application of Generalized Diagonal Dominance to Linear System Stability Theory. Int. J. Control, $36,185-212$

McAvoy, T. J. (1981). Connection Between Relative Gain and Control Loop Stability and Design. AIChE J., 27, 613-619.

Mees, A. I. (1981). Achieving Diagonal Dominance. Systems and 
Control Letters, 1, 155-158.

Postlethwaite, I. and J. M. MacFarlane (1979). A Complex Variable Approach to the Analysis of Linear Multivariable Feedback Systems. Springer, Berlin.

Rijnsdorp, J. E. (1965). Interaction in Two-Variable Control Systems for Distillation Columns - I. Automatica, 1, 15-28.

Rosenbrock, H. H. (1974). Computer-Aided Control System Design. Academic Press, London.

Seneta, E. (1973). Non-Negative Matrices, John Wiley \& Sons, New York. Shinskey, F. G. (1979). Process Control Systems. McGraw-Hill Book Co., New York.

Tung, L. S. and T. F. Edgar (1981). Analysis of Control-Output Interactions in Dynamic Systems. AIChE J., 28 590-693. Witcher, M. F. and T. J. McAvoy (1977). Interacting Control Systems: Steady State and Dynamic Measurement of Interaction. ISA Trans., $16,34-41$

Woolverton, P. F. (1980). How to Use Relative Gain Analysis in Systems with Integrating Variables. InTech, September, 63-65. 
160

Table 1. Liquid-Liquid Separator Transfer Function Matrix

\begin{tabular}{lcc}
$19.9 g_{11}(s)$ & $98.6 g_{12}(s)$ & $101.5 g_{13}(s)$ \\
$1.435 g_{21}(s)$ & $-3.3 g_{22}(s)$ & $-3.025 g_{23}(s)$ \\
0 & $\frac{145.5}{s} g_{32}(s)$ & $\frac{-4.12}{s} g_{33}(s)$ \\
\hline
\end{tabular}

Table 2. Distillation Column Transfer Function Matrix

\begin{tabular}{lccc}
\hline & reflux ratio & side draw & reboil duty \\
\hline toluene in bottom & $\frac{0.374 e^{-7 \cdot 75 s}}{(22.2 s+1)^{2}}$ & $\frac{-11.3 e^{-3 \cdot 79 s}}{(21.74 s+1)^{2}}$ & $\frac{-9.811 e^{-1.59 s}}{(11.36 s+1)}$ \\
toluene in tops & $\frac{-1.986 e^{-0.71 S}}{(66.67 s+1)^{2}}$ & $\frac{5.24 e^{-60 S}}{(400 s+1)}$ & $\frac{5.984 e^{-2 \cdot 24 S}}{(14.29 s+1)}$ \\
benzene in side draw & $\frac{0.0204 e^{-0.59 S}}{(7.14 s+1)^{2}}$ & $\frac{-0.33 e^{-0.68 s}}{(2.38 s+1)^{2}}$ & $\frac{2.38 e^{-0.42 S}}{(1.43 s+1)^{2}}$ \\
\hline
\end{tabular}


FIGURE CAPTIONS

Figure 1. General decentralized control structure.

Figure 2. Block-diagram representation of interactions as additive uncertainty.

Figure 3. Rearranged classical control structure.

Figure 4. (Example 1), Interaction Measures. Line $1=\mu^{-1}(E(j \omega))$ for decentralized controller; Line $2=\mu^{-1}(\mathbf{E}(j \omega))$ and Line $3=$ $\rho^{-1}(|E(j \omega)|)$ for block-decentralized controller.

Figure 5. (Example 2), Line $1=\mu^{-1}(E(j \omega))$ for fully-decentralized controller; Line $2=\mu^{-1}(E(j \omega))$ for block-decentralized controller.

Figure 6. (Example 3), Line $1=\mu^{-1}(E(j \omega))$; Lines 2, 3 and $4=$ amplitude ratio for (57), (58) and (59), respectively.

Figure 7a. (Example 3), Closed-loop responses for unit step change in $r_{2}(s) . \tilde{h}_{i}(s),(i=1,3)$, given by $(57)$.

Figure 7b. (Example 3), Closed-10op responses for unit step change in $r_{2}(s) . \tilde{h}_{i}(s),(i=1,3)$, given by (58).

Figure 7c. (Example 3), Closed-1oop responses for unit step change in $r_{2}(s), \tilde{h}_{i}(s),(i=1,3)$, given by $(59)$.

Figure 8. (Example 4), Line $1=\mu^{-1}(E(j \omega) \mathbf{W}(j \omega))$; Line $2=\mu^{-1}(E(j \omega))$.

Figure 9. (Example 5), Bode plots. Line $1=\kappa(s)$; Line $2=z(s)=$ $\tilde{\mathrm{h}}_{1}(\mathrm{~s}) \tilde{\mathrm{h}}_{2}(\mathrm{~s}) K(\mathrm{~s})$.

Figure 10. (Example 5), closed-1oop response for unit step change in $r_{1}(s)$, diagonal pairings.

Figure 11. (Example 5), closed-1oop response for unit step change in $r_{1}(s)$, of $f$-diagonal pairings. 


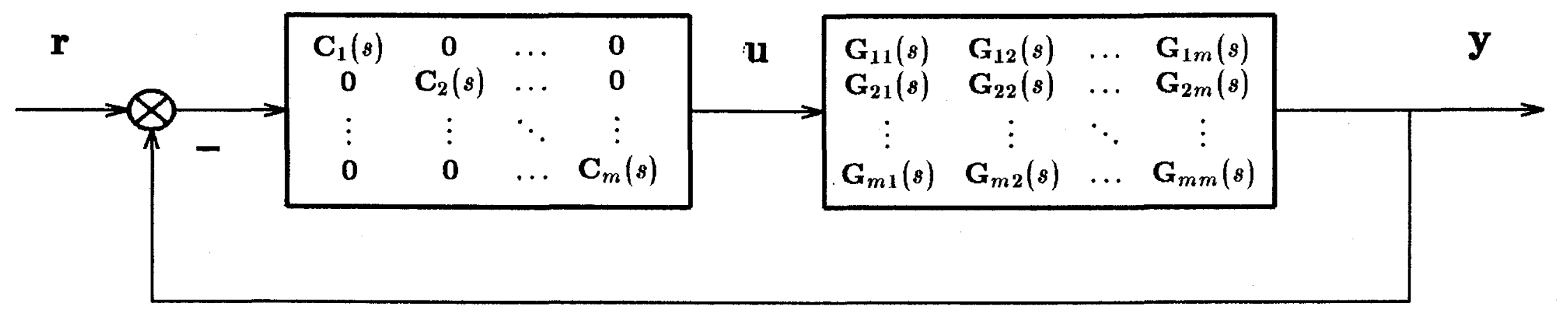

Figure 1. General decentralized control structure. 


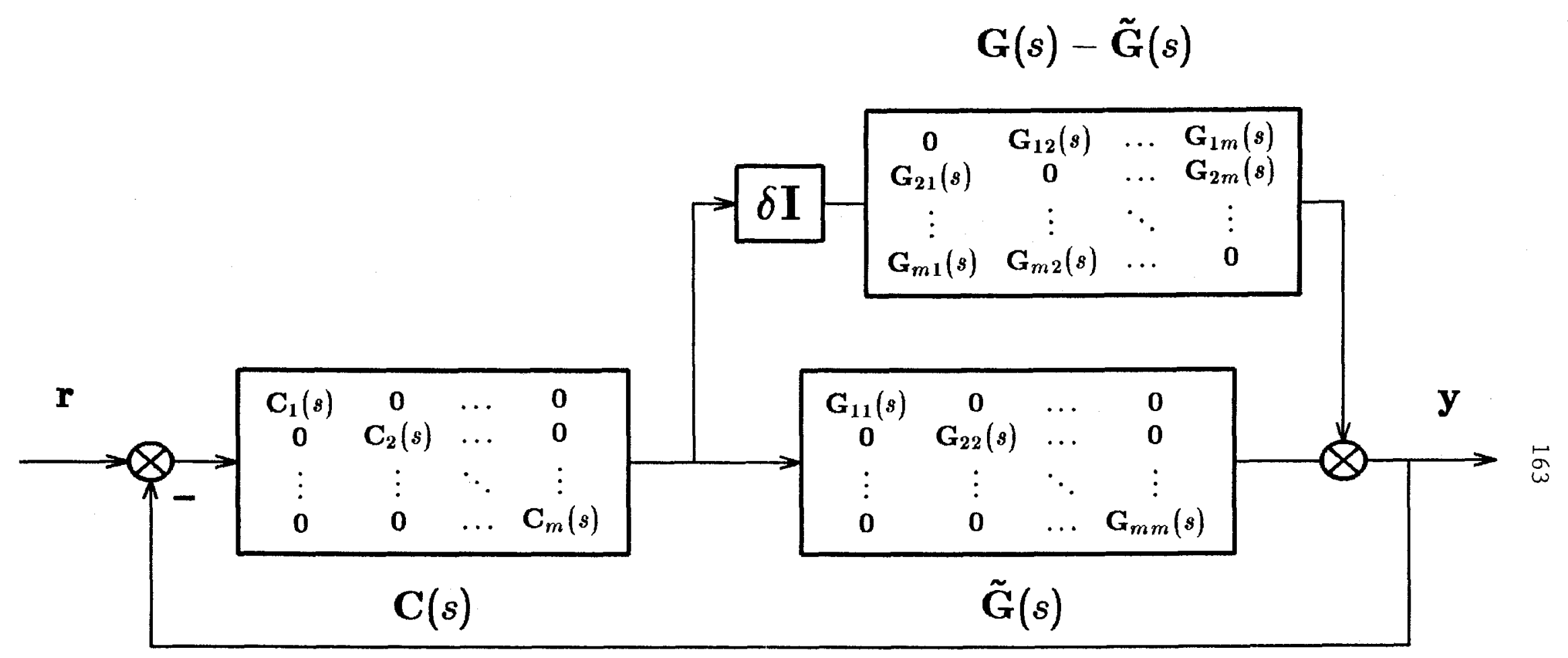

Figure 2. Block-diagram representation of interactions as additive uncertainty. 


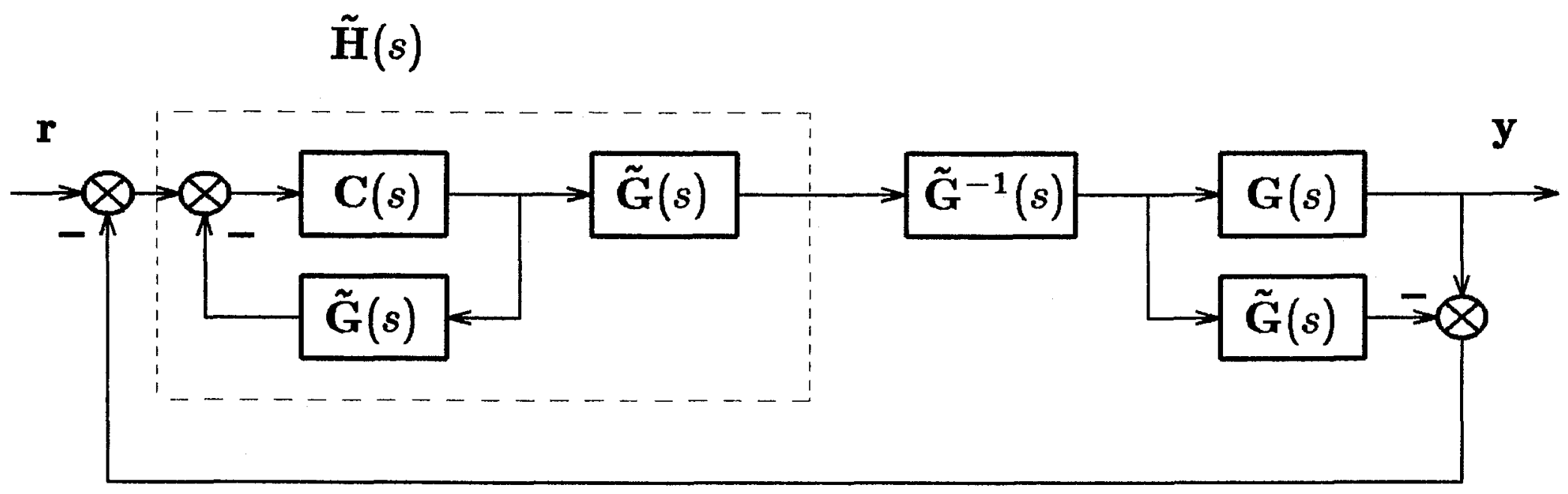

Figure 3. Rearranged classical control structure. 


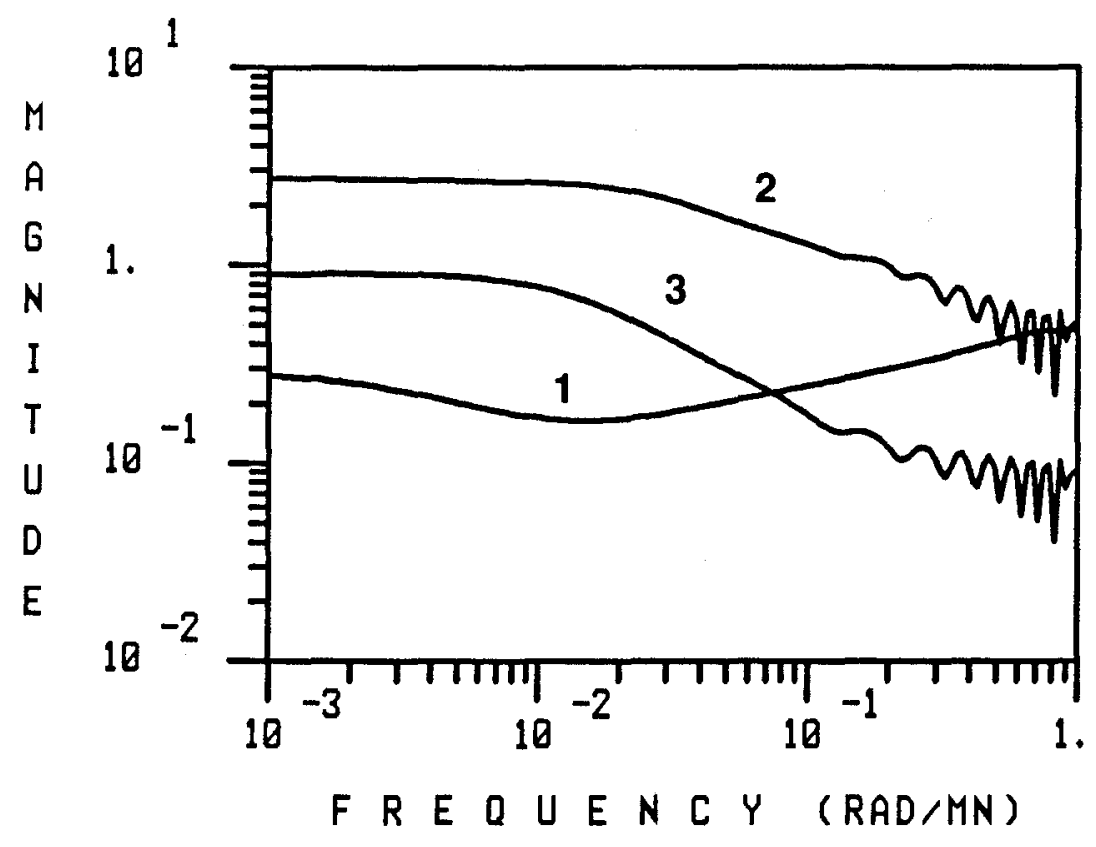

Figure 4. (Example 1), Interaction Measures. Line $1=\mu^{-1}(E(j \omega)$ ) for decentralized controller; Line $2=\mu^{-1}(E(j \omega))$ and Line $3=$ $\rho^{-1}(|E(j \omega)|)$ for block-decentralized controller. 


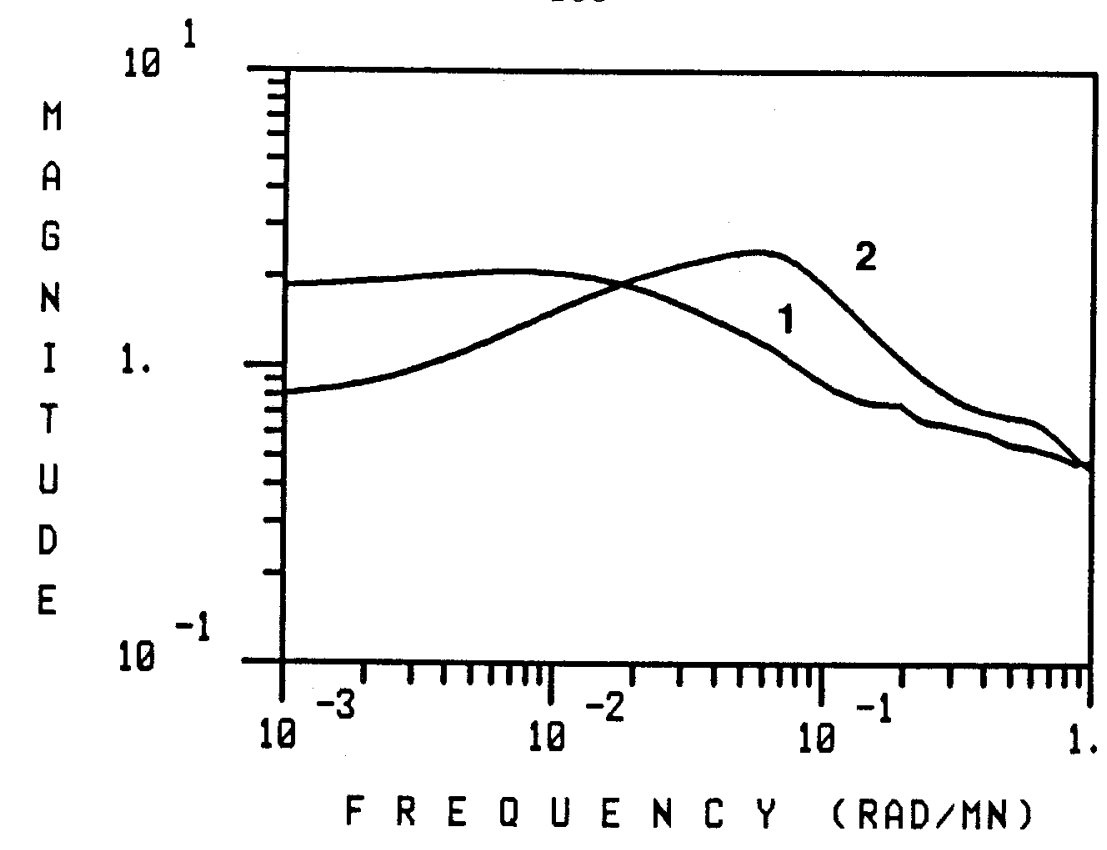

Figure 5. (Example 2), Line $1=\mu^{-1}(E(j \omega))$ for fully-decentralized controller; Line $2=\mu^{-1}(E(j \omega))$ for block-decentralized controller.

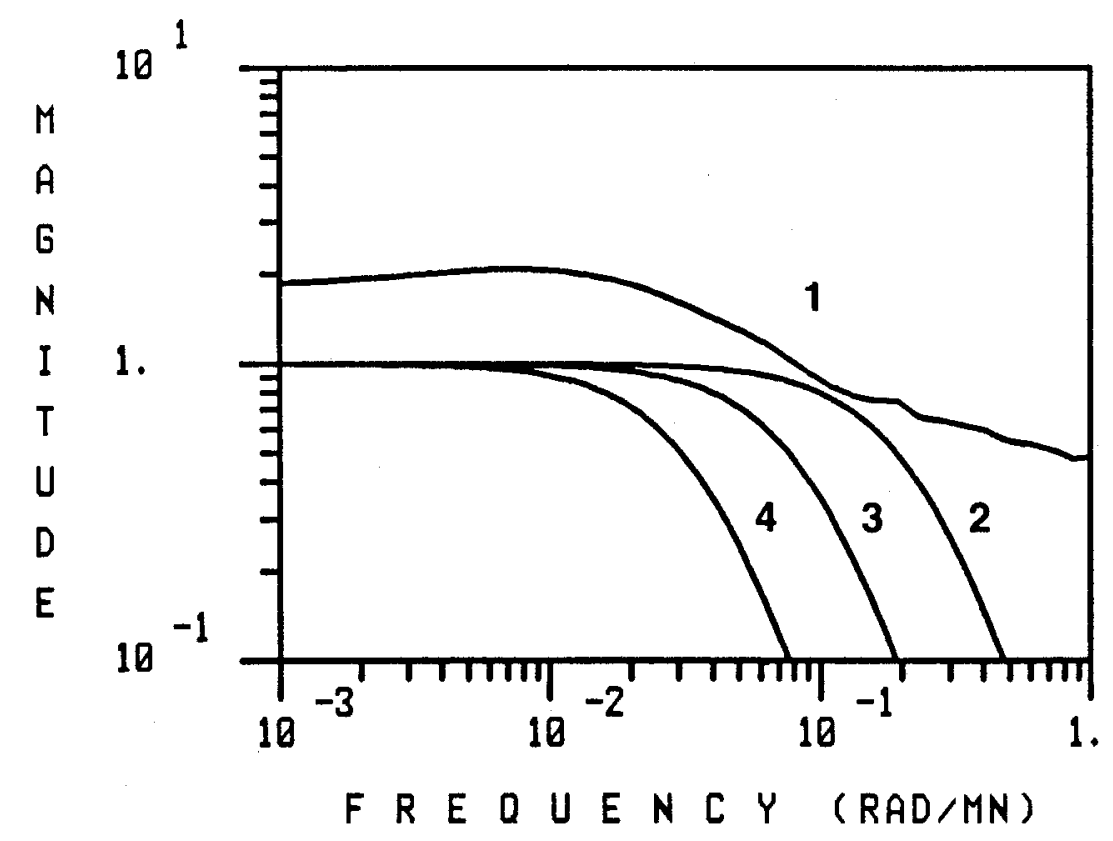

Figure 6. (Example 3), Line $1=\mu^{-1}(E(j \omega))$; Lines 2,3 and $4=$ amplitude ratio for (57), (58) and (59), respectively. 


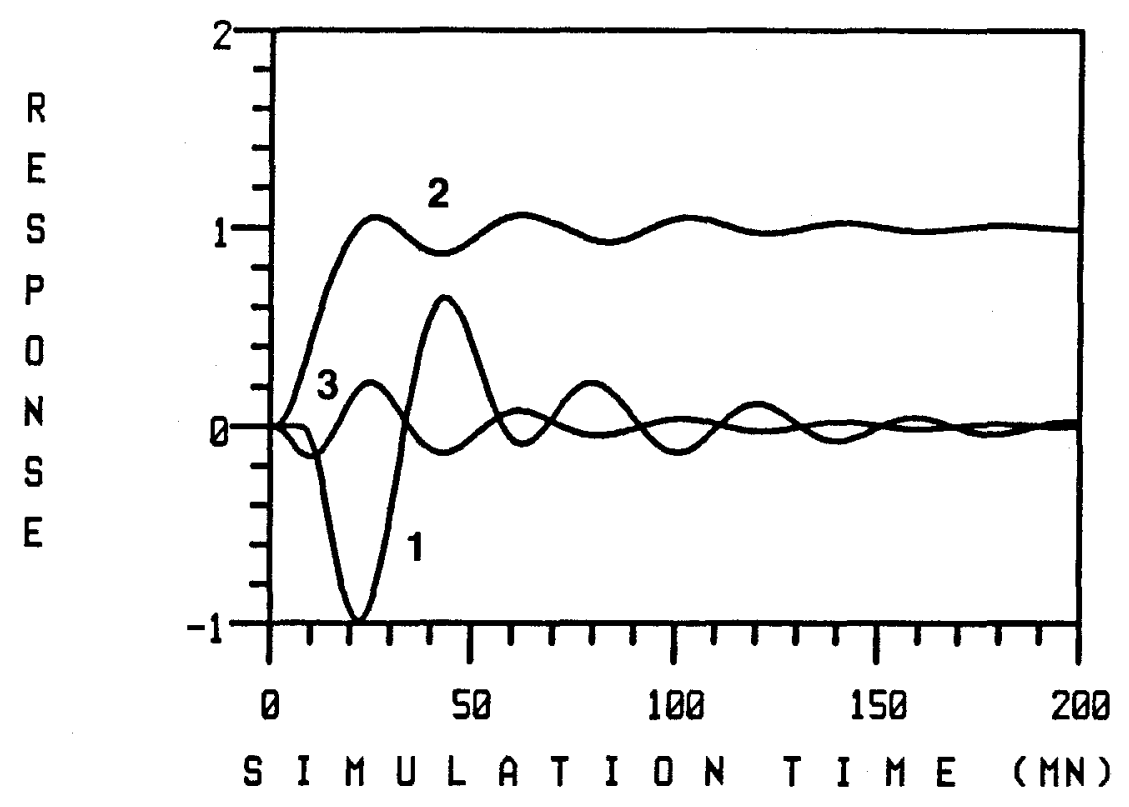

Figure 7a. (Example 3), Closed-1oop responses for unit step change in $r_{2}(s)$. $\tilde{h}_{\mathfrak{i}}(s),(i=1,3)$, given by $(57)$.

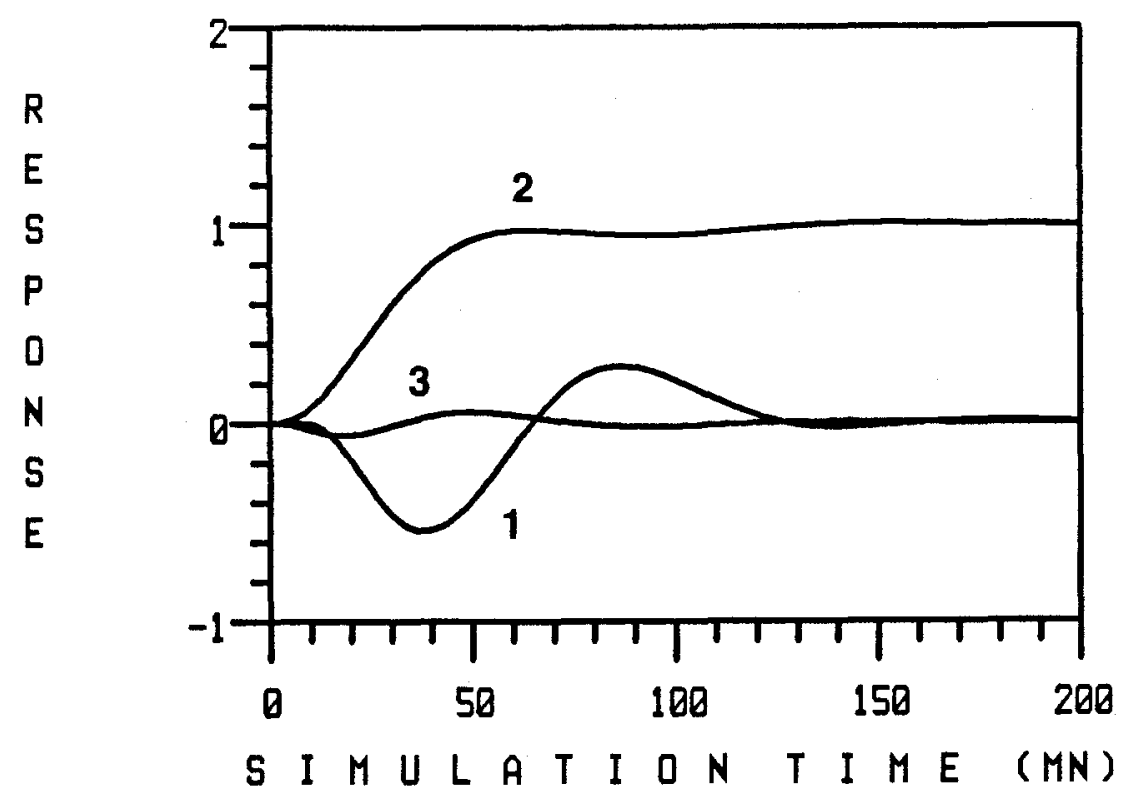

Figure 7b. (Example 3), Closed-10op responses for unit step change in $r_{2}(s) . \quad \tilde{h}_{i}(s),(i=1,3)$, given by $(58)$. 


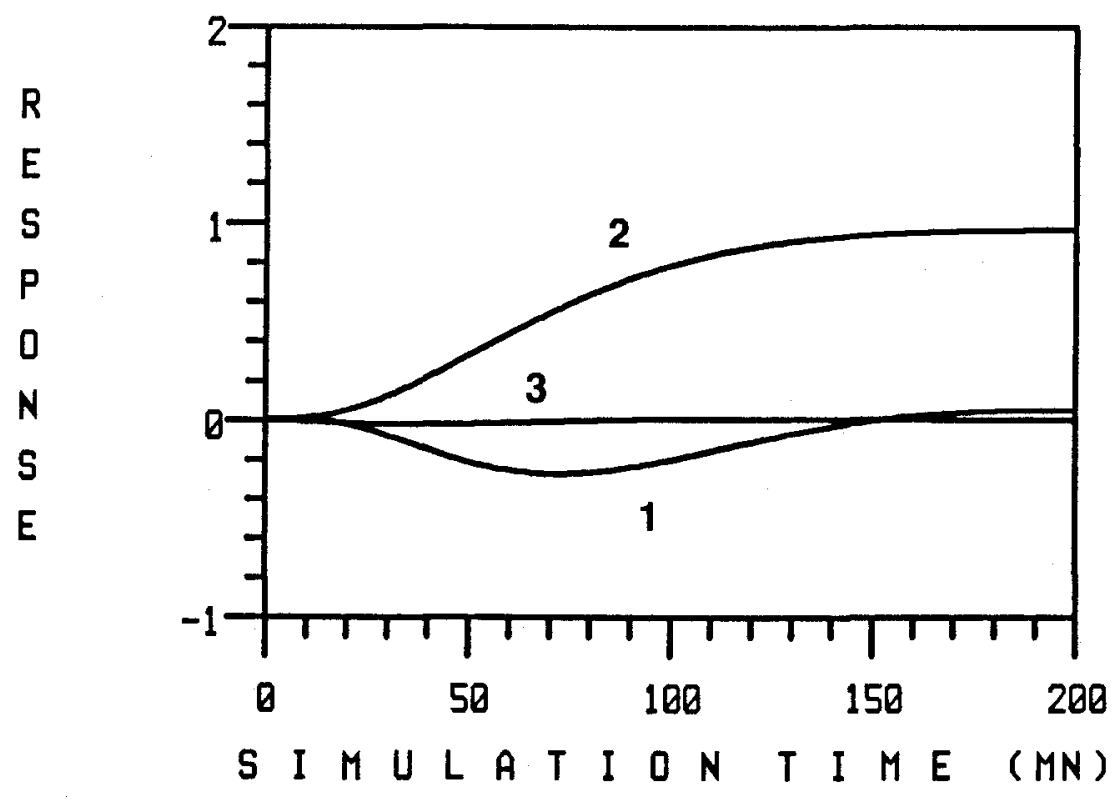

Figure 7c. (Example 3), Closed-10op responses for unit step change in $r_{2}(s) . \quad \tilde{h}_{i}(s),(i=1,3)$, given by $(59)$.

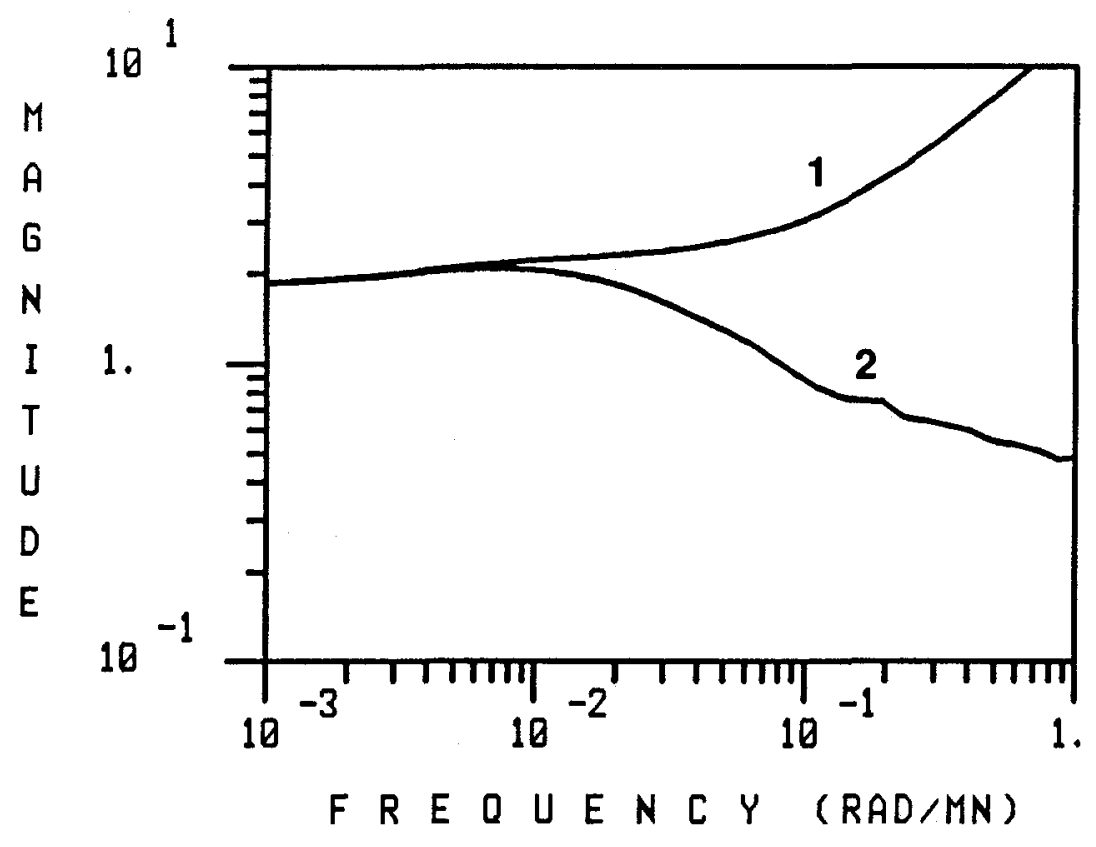

Figure 8. (Example 4), Line $1=\mu^{-1}(E(j \omega) \mathbf{W}(j \omega))$; Line $2=\mu^{-1}(E(j \omega))$. 

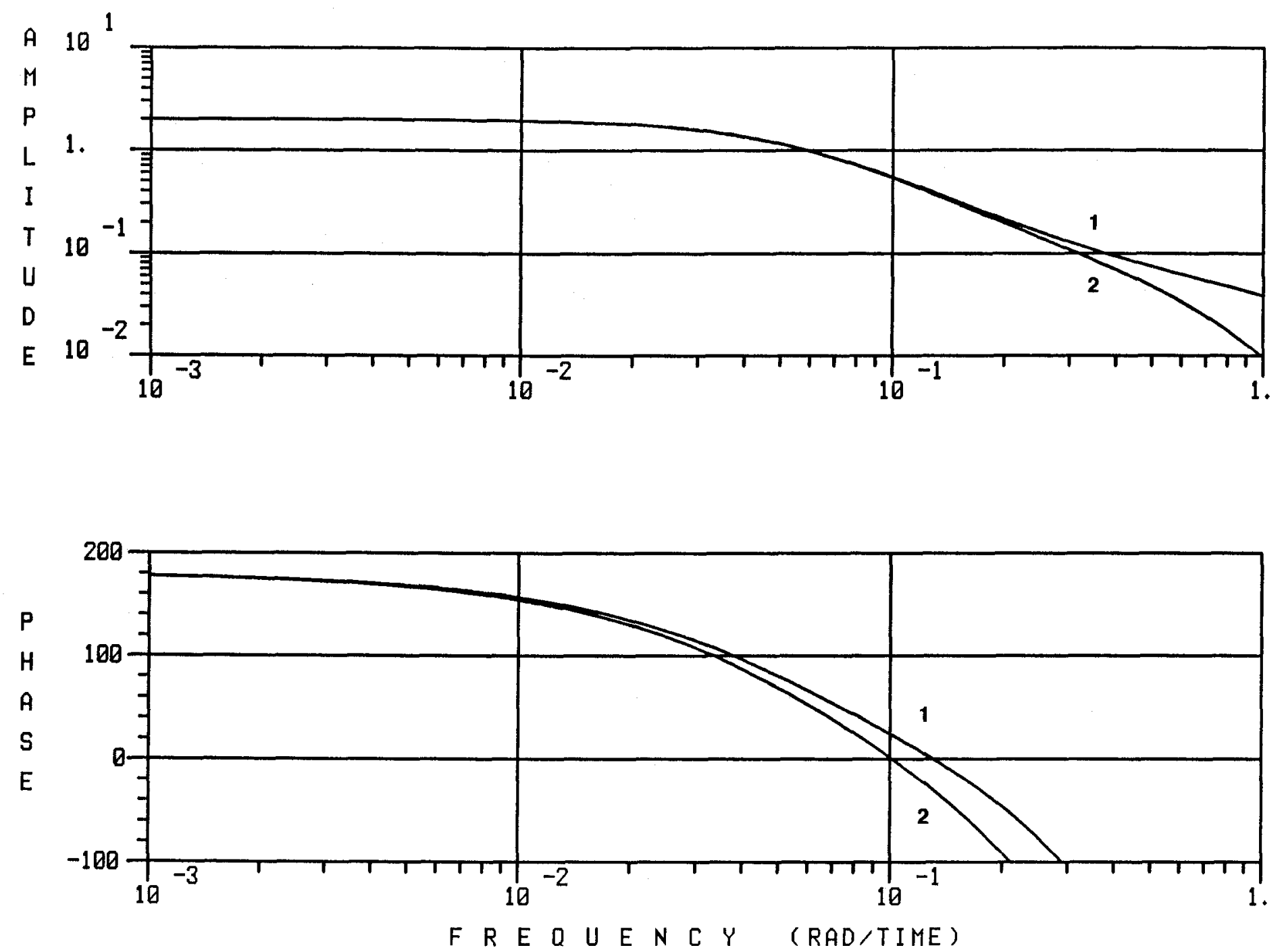

Figure 9. (Example 5), Bode plots. Line $1=\kappa(s)$; Line $2=z(s)=h_{1}(s) h_{2}(s)_{k}(s)$. 


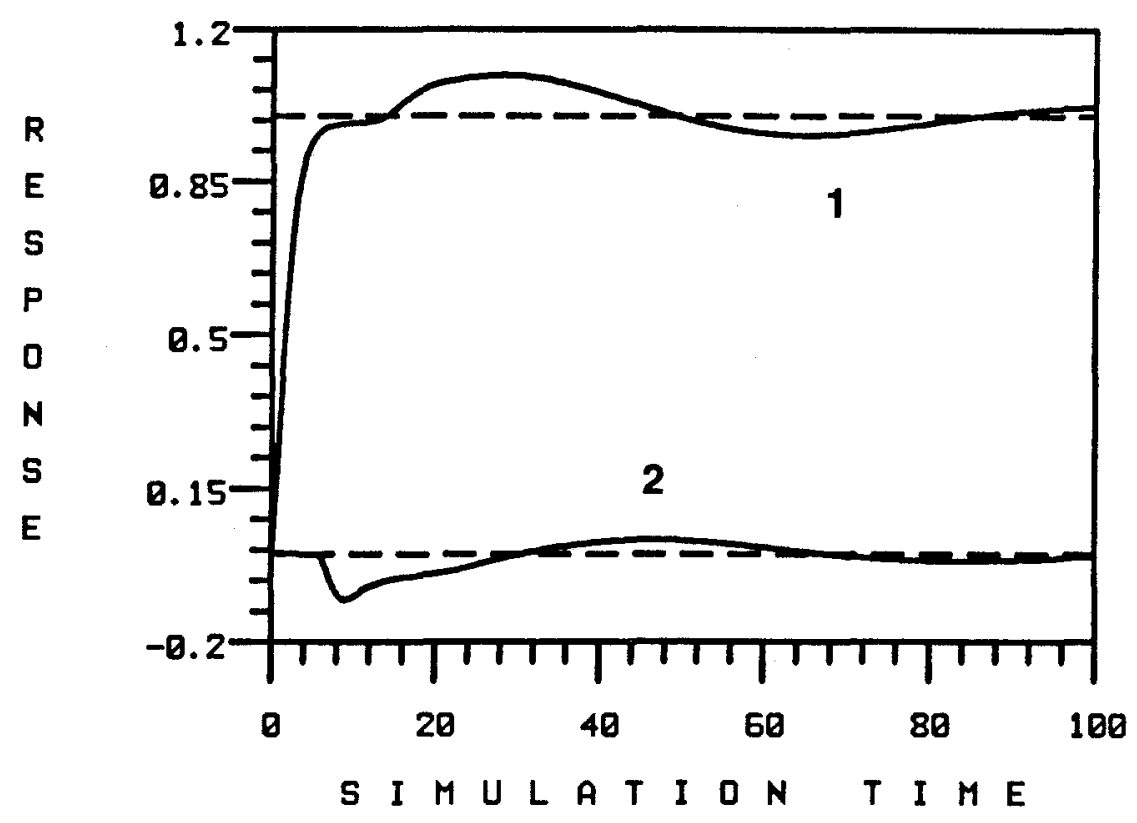

Figure 10. (Example 5), closed-10op response for unit step change in $r_{1}(s)$, diagonal pairings.

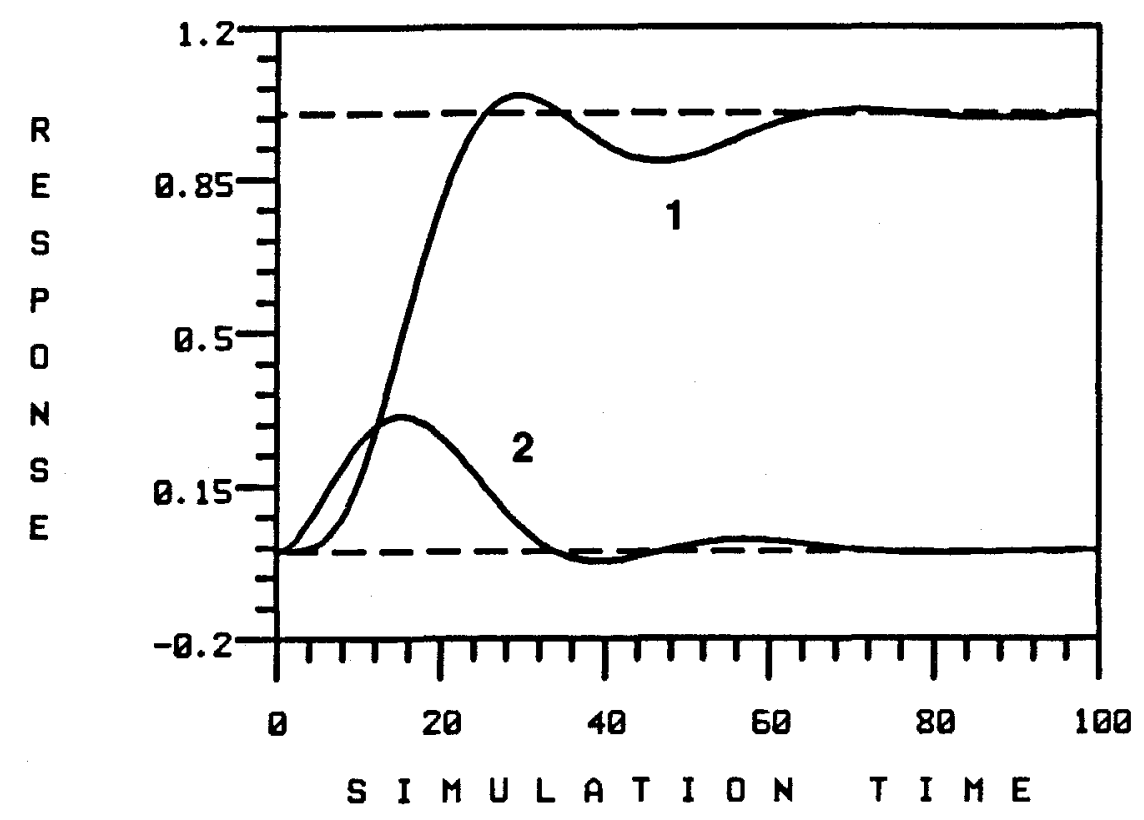

Figure 11. (Example 5), closed-1oop response for unit step change in $r_{1}(s)$, off-diagonal pairings. 
CHAPTER V: A COMPUTER-AIDED METHODOLOGY FOR THE DESIGN OF DECENTRALIZED CONTROLLERS 


\section{INTRODUCTION}

Most multivariable controllers in the chemical process industries have only limited access to plant inputs and outputs. Reordering these variables always makes it possible to present the controller in a diagonal or block-diagonal form (Fig. 1)

$$
C(s)=\operatorname{diag}\left(C_{1}(s), C_{2}(s), \ldots C_{m}(s)\right)
$$

with

$$
u_{i}=c_{i}(s)\left(y_{i}-r_{i}\right)
$$

Here, it is assumed that the vectors of inputs, outputs and setpoints have been partitioned in the same manner $u=\left(u_{1}, u_{2}, \ldots u_{m}\right)^{T}, y=$ $\left(\mathrm{y}_{1}, \mathrm{y}_{2}, \ldots \mathrm{y}_{\mathrm{m}}\right)^{\mathrm{T}}$ and $\mathbf{r}=\left(\mathrm{r}_{1}, \mathrm{r}_{2}, \ldots \mathrm{r}_{\mathrm{m}}\right)^{\mathrm{T}}$, respectively.

In this communication, we shall consider any square, nxn, open loop stable system $\mathrm{G}(\mathrm{s})$ under feedback with such "decentralized" controllers. In all subsequent developments, it will be assumed that the controllers $\mathrm{C}(\mathrm{s})$ contain integral action, i.e., $\mathrm{H}(\mathrm{s}=\mathrm{O})=\mathrm{H}(0)=\mathrm{I}$, where $\mathbf{H}(\mathrm{s})=$ $G(s) C(s)(I+G(s) C(s))^{-1}$ denotes the closed loop transfer function matrix for the system in Fig. 1. Each controller block $\mathrm{C}_{i}(\mathrm{~s})$ can therefore be decomposed into a matrix of integrators $\underline{k_{i}} / s \cdot I_{i}$ and a compensator $\operatorname{matrix} \mathrm{K}_{\mathrm{i}}(\mathrm{s})$ (Fig. 2).

With respect to the system shown in Fig. 1, the following notation will be adopted: Let I denote any subset of integers within the set $\{1,2, \ldots \mathrm{m}\}$, where $\mathrm{m}$ is the number of blocks $\mathrm{C}_{j}(\mathrm{~s})$ in $\mathrm{C}(\mathrm{s})$, and let $\mathfrak{g}$ be the ensemble of all possible I's, The subsets I are used to define the subplants

$$
\mathrm{G}_{\mathrm{I}}(\mathrm{s})=\left\{\mathbf{G}_{i j}(\mathrm{~s})\right\}, \mathrm{i}, j \in \mathrm{I}
$$

within $G(s)$. For example, if $m=3$ and $I=\{1,3\}$, then 


$$
G_{I}(s)=\left[\begin{array}{ll}
G_{11}(s) & G_{13}(s) \\
G_{31}(s) & G_{33}(s)
\end{array}\right] .
$$

Definitions of the submatrices $C_{I}(s)$ and $H_{I}(s)=G_{I}(s) C_{I}(s)\left(I+G_{I}(s) C_{I}(s)\right)^{-1}$ follow accordingly. $\mathrm{H}_{\mathrm{I}}(\mathrm{s})$ denotes the closed loop transfer matrix when only the controller blocks $\mathrm{C}_{i}(\mathrm{~s})$ ( $\forall i \varepsilon I$ ) are placed in automatic, all other blocks being in manual. In the event where $I=\{i\}$, the simpler notation

$$
H_{I}(s)=H_{i}(s)=G_{i i}(s) C_{i}(s)\left(I+G_{i i}(s) C_{i}(s)\right)^{-1}
$$

will be used. Finally, if $\mathrm{G}_{i j}(\mathrm{~s})$ has only one input and one output, $G_{i i}(s)=g_{i i}(s)$ and $H_{i}(s)=h_{i}(s)$.

Decentralized controllers have been preferred to more complex multivariable controllers for the following reasons:

(i) Ease of implementation. The control system has fewer communication links.

(ii) Simplified design. The controller has fewer tuning parameters.

(iii) Decentralized tuning. The controllers $C_{i}(s),(i=1, m)$ are tuned to make each block $\mathrm{H}_{\mathrm{i}}(\mathrm{s})$ stable.

(iv) Failure tolerance. The control system must remain stable in the event of loop failure. This problem cannot be addressed in a simple manner with full controllers.

The last three reasons for using decentralized controllers, simplified design, decentralized tuning and failure tolerance, require more precise specifications. A decentralized control system for a plant G(s) will be justified if it possesses the following properties:

Property 1. Stability. $\mathbf{H}(\mathrm{s})$ and $\mathrm{H}_{\mathrm{i}}(\mathrm{s}),(\mathbf{i}=1, \mathrm{~m})$ are stable and track asymptotically constant inputs with vanishing error, i.e., $\mathrm{H}(0)=\mathrm{I}$ and $H_{i}(0)=I,(i=1, m)$. 
Property 2. Integral Controllability. For simple tuning, the control system must be integral controllable in the sense defined by Grosdidier et al. (1985): With reference to Fig. 2, assume $\underline{k}_{i}=\underline{k}, \forall i$. The openloop stable system $Q(s)=G(s) K(s)$ is Integral Controllable (IC) if there exists a $\underline{k}^{*}>0$ such that the closed loop system shown in Fig. 2 is stable for all values of $\underline{k}$ satisfying $0<\underline{k} \leq \underline{k}^{*}$ and has zero tracking error for asymptotically constant disturbances.

The control system must also be IC with respect to each of the controller blocks $C_{j}(s)$. Block IC refers to the ability to selectively detune one of the controller gains $\underline{k}_{i}$. Let $I_{i}=\{1,2, \ldots i-1, i+1, \ldots m\}$ and assume $\mathrm{H}_{\mathrm{I}_{1}}(\mathrm{~s})$ is stable. The open-loop stable system $\mathrm{Q}(\mathrm{s})=\mathrm{G}(\mathrm{s}) \mathrm{K}(\mathrm{s})$ is block-i IC if there exists a $\underline{k}_{i}^{*}>0$ such that the closed loop system shown in Fig. 2 is stable for all values of $\underline{k}_{i}$ satisfying $0<\underline{k_{i}} \leq{\underline{k_{i}}}^{*}$ and has zero tracking error for asymptotically constant disturbances. Property 3. Failure tolerance. The control system must remain stable when failure occurs in one or more of the feedback loops. In this event, it is assumed that the failures are recognized and the corresponding controller blocks are placed in manual. For stability under any combination of loop failures, we require $\mathrm{H}_{\mathrm{I}}(\mathrm{s})$ stable, $\forall I \mathcal{J}$.

Whether or not a decentralized control system possesses these three properties depends on the plant, the control structure and on controller tuning. By control structure we mean the number and size of the controller blocks and the input/output pairings corresponding to each of these. In the present context, a control structure will be called "acceptable" if it can lead to a decentralized control system which possesses Properties (1)-(3). 
Obviously, these properties are quite demanding. In some cases, interactions in the plant will be such that it will not be possible to satisfy them all. In other cases, they will be satisfied only with a significant sacrifice of closed-1oop performance.

Interaction Measures (IM's) are analysis tools which aid both in control structure selection and controller tuning. They are conditions on $G(s), G(0)$ and $G(s) C(s)$ which indicate whether properties (1)-(3) are or can be satisfied, and under what conditions. Note that since Properties (1)-(3) are scaling invariant, it is only logical to expect that these IM's are also scaling invariant. By scaling, we mean pre- and post-multiplication of the matrix $G(s)$ by diagonal, non singular matrices.

The objective of this communication is to present a synopsis of currently existing IM's and to show how these can form the basis of a computer aided methodology for the design of decentralized controllers.

\section{SYNOPSIS OF INTERACTION MEASURES}

The emphasis in this section will be placed on presenting each tool's theoretical properties and on showing how these relate to properties (1)-(3). Most results will be stated without proof since these can be found in the cited references.

The Relative Gain Array (Bristol, 1966). Currently the most widely used measure of interactions, the Relative Gain Array (RGA) is a pairing tool for diagonal control structures. For each possible pair $\left(u_{j}, y_{i}\right)$ of input and output variables in a control system, a Relative Gain (RG) is defined 


$$
\lambda_{i j}=g_{i j}(0) \cdot \hat{g}_{j i}(0) .
$$

Here $g_{i j}(0)$ and $\hat{g}_{j i}(0)$ are the $(i, j)$ th and $(j, i)$ th elements of the steady state gain matrices $\mathrm{G}(0)$ and $\mathrm{G}^{-1}(0)$. The $\mathrm{RGA}$ is defined as the real, scaling invariant matrix

$$
\Lambda=\left\{\lambda_{i j}\right\}, i, j=1, n
$$

A number of rigorous theoretical results are associated with the RGA. The following theorems were taken from Grosdidier et al., (1985). Theorem 1. Stability. If $\lambda_{i i}<0$, then for any compensator $\mathbf{K}(\mathbf{s})=$ $\operatorname{diag}\left(k_{1}(s), k_{2}(s), \ldots k_{m}(s)\right)$ with the properties

(a) $\mathrm{G}(\mathrm{s}) \mathrm{K}(\mathrm{s})$ is proper,

(b) $\quad k_{i j}(s)=k_{j i}(s)=0, \quad \forall_{j} \neq i$

and any $\underline{k}_{i}>0, \forall i$, the closed loop system shown in Fig. 2 has at least one of the following properties:

(a) The closed loop system is unstable.

(b) Loop i is unstable by itself, i.e., with all the other loops opened.

(c) The closed loop system is unstable as $100 \mathrm{p} j$ is removed. Theorem 1 says that a diagonal control structure can satisfy properties (1) and (3) only if it is based on variable pairings with positive RG's. The weakness of this result is that for systems larger than $2 \times 2$ these properties still may not be satisfied when all the RG's are positive. A further limitation of the RGA is that it does not address multiple loop failures as required by property (3). Theorem 2. Robustness. For a $2 \times 2$ transfer matrix $G(0)$, the minimum condition number $\gamma^{*}$ is given

$$
\gamma^{*}=|| \Lambda||_{1}+\sqrt[V]{\mid \Lambda \|_{1}^{2}-1}
$$

where ||$\Lambda||_{1}$ denotes the 1 -norm of the RGA. For nxn plants, the equality 
is replaced by the approximate bound (Skogestad, 1985)

$$
r^{*} \leq|| \Lambda|| E S
$$

where ||$\Lambda|| E S=\sum_{i, j}\left|\lambda_{i j}\right|$ denotes the element sum norm of the RGA.

Theorem 2 shows that the RGA is rigorously related to the model error sensitivity of a plant - as quantified by its minimal condition number $\gamma^{*}$. Systems with large RG's, and therefore large $\gamma^{*}$, are sensitive to modeling errors and are difficult to control regardless of what control strategy might be used.

Block Relative Gains. Block Relative Gains (BRG's), the multivariable extension of RG's, were recently documented by Manousiouthakis and Arkun (1985). The BRG of a square subsystem $G_{i j}(s)$ in $G(s)$ is defined as

$$
\Lambda_{i j}=G_{i j}(0)=\hat{G}_{j i}(0)
$$

where $\hat{G}_{j i}(0)$ is the $(j, i)$ th block of the matrix $G^{-1}(0)$.

In an $n \times n$ plant $G(s)$, the total number of different subsystems $\mathrm{G}_{i j}(\mathrm{~s})$ with dimension $\ell \mathrm{x} \ell$ is $\left[\begin{array}{l}\mathrm{n} \\ \ell\end{array}\right]^{2}$, each subsystem corresponding to a different subset of input and output variables. Accordingly, an equal number of BRG's can be calculated, and this precludes an array-like display.

Although the simplicity of the RGA is lost, the usefulness of BRG'S remains the same. This next theorem is a generalization of Theorem 1 . Let $\operatorname{det}(\mathbf{A})$ denote the determinant of the matrix $\mathbf{A}$.

Theorem 3. If $\operatorname{det}\left(\Lambda_{i i}\right)<0$, then for any compensator $\mathbf{K}(\mathrm{s})$ with the properties

(a) $\mathbf{G}(\mathrm{s}) \mathbf{K}(\mathrm{s})$ is proper, 
(b) $\quad K_{i j}(s)=K_{j i}(s)=0 \quad \forall j \neq i$

and any $\underline{k}_{i}>0, \forall i$, the closed loop system shown in Fig. 2 has at least one of the following properties:

(a) The closed loop system is unstable.

(b) Block $i$ is unstable by itself, i.e., with all the other blocks of loops opened.

(c) The closed loop system is unstable as block i is removed.

Proof: See the Appendix.

Block diagonal control systems will satisfy Properties (1) and (3) only if their associated BRG's have positive determinants. For simplicity, a BRG with positive determinant will subsequently be referred to as a positive BRG. Note that although BRG's are input and output scaling dependent, their useful property $\left(\operatorname{det}\left(\Lambda_{i i}\right)\right)$ is not.

Niederlinski's Index (Grosdidier et al., 1985). Like the RGA, Niederlinski's Index (NI) is based on steady state gain information and is a tool for control structure selection only. Initially defined for diagonal control structures, the Index was later generalized to blockdiagonal structures (Grosdidier and Morari, 1986). It is defined as

$$
\operatorname{det}\left(\mathbf{G}(0) \tilde{\mathbf{G}}^{-1}(0)\right)
$$

where

$$
\tilde{G}(s)=\operatorname{diag}\left(G_{11}(s), G_{22}(s), \ldots G_{m m}(s)\right) .
$$

The theoretical justification for NI is based on the following theorem (Grosdidier and Morari, 1985).

Theorem 4. Assume that

(a) $\quad \mathbf{G}(\mathrm{s})$ and $\tilde{\mathbf{H}}(\mathrm{s})=\tilde{\mathrm{G}}(\mathrm{s}) \mathbf{C}(\mathrm{s})(\mathbf{I}+\tilde{\mathrm{G}}(\mathrm{s}) \mathbf{C}(\mathrm{s}))^{-1}$ are strictly stable,

(b) $\mathrm{G}(\mathrm{s}) \mathrm{C}(\mathrm{s})$ is strictly proper,

(c) $\tilde{\mathrm{H}}(\mathrm{s})$ has vanishing tracking error for asymptotically constant 
inputs, i.e., $\tilde{\mathrm{H}}(0)=I$.

Then the closed loop system $\mathrm{H}(\mathrm{s})$ will be unstable if

$$
\operatorname{det}\left(\mathbf{G}(0) \cdot \tilde{G}^{-1}(0)\right)<0 .
$$

Theorem 4 says that property (1) can be satisfied only if $\operatorname{det}\left(\mathbf{G}(0) \tilde{G}^{-1}(0)\right)>0$. Like the RGA, NI is scaling invariant.

Integral Controllability Eigenvalues. The conditions under which a system $Q(s)=G(s) K(s)$ (Fig. 2) is IC were derived by Morari (1985). Theorem 5. Assume $\underline{k}_{j}=\underline{k}, \forall i$. The rational system $Q(s)$ is IC if and only if all the eigenvalues of the matrix $G(s) K(0)$ lie in the open right half complex plane. The rational system $Q(s)$ is not IC if any of the eigenvalues of $\mathrm{G}(0) \mathrm{K}(0)$ lie in the open left half complex plane.

This next theorem follows from Theorem 5 and the concept of BRG. Theorem 6. Assume $\mathrm{H}_{\mathrm{I}_{\mathrm{i}}}(\mathrm{s})$ is stable. The rational system $\mathrm{Q}(\mathrm{s})=\mathrm{G}(\mathrm{s}) \mathrm{K}(\mathrm{s})$ is Block-i IC if and only if all the eigenvalues of the matrix $\Lambda^{-1}$ ii $\mathrm{G}_{\mathrm{ij}}(0) \mathrm{K}_{\mathrm{i}}(0)$ lie in the open right half complex plane. The rational system $Q(s)$ is not Block-i IC if any of the eigenvalues of $\Lambda^{-1}$ ii $\mathrm{G}_{\mathrm{ii}}(0) \mathrm{K}_{\mathbf{i}}(0)$ lie in the open left half complex plane. Proof: See the Appendix.

Theorems 5 and 6 state necessary and sufficient conditions for property (2). Note that these conditions are scaling invariant only if care is taken to scale $\mathbf{C}(\mathrm{s})$ consistently with $\mathbf{G}(\mathrm{s})$. Their main drawback is that they require the user to assume steady-state values for the compensators $K_{i}(s),(i=1, m)$.

The Direct Nyquist Array (Rosenbrock, 1974). The Direct Nyquist Array (DNA) methodology was the first attempt to formulate a rigorous theory of decentralized control. Initially introduced as a tool for the design of diagonal controllers, the method was later generalized to 
block-diagonal controllers (Limebeer, 1982). It can be used both for control structure selection and controller tuning. The "generalized" or optimally scaled version of the DNA will be the only one discussed in this publication.

This next theorem is valid only for diagonal controllers. Theorem 7. Assume that $\mathbf{G}(\mathrm{s})$ and $\tilde{\mathrm{H}}(\mathrm{s})$ are stable. Then the closed 1oop system $H(s)$ is stable if

$$
\left|h_{i}(j \omega)\right|<\rho^{-1}\left(\left|(\mathbf{G}(j \omega)-\tilde{G}(j \omega)) \tilde{G}^{-1}(j \omega)\right|\right) \quad \forall i, \omega
$$

where $\tilde{G}(s)=\operatorname{diag}(\mathbf{G}(s))$. In $(14), \rho(A)$ denotes the spectral radius of the matrix $\mathbf{A}$ and $|\mathbf{A}|$ is the matrix $\mathbf{A}$ with all its elements replaced by their absolute values. The DNA IM (cf. $\left.\rho\left(\left|(\mathbf{G}(j \omega)-\tilde{G}(j \omega)) \tilde{G}^{-1}(j \omega)\right|\right)\right)$ expresses the constraint imposed on the closed loop transfer functions $h_{i}(j \omega),(i=1, n)$ which guarantees that the full system is stable.

Theorem 7 is actually a reformulation of the original result by Rosenbrock (1974). It should more accurately be seen as an optimally scaled version of the IMC IM (Economou and Morari, 1986). The DNA IM is simply the optimally scaled 1-norm of the matrix $\mathbf{E}(j \omega)=$ $(\mathbf{G}(j \omega)-\tilde{G}(j \omega)) \tilde{G}^{-1}(j \omega)$. It is also known as the Perron-Frobenius root of the matrix $|\mathbf{E}(j \omega)|$ (Mees, 1981), and is a quantity invariant under input and output scaling. A decentralized control system designed on basis of Theorem 7 can always be made to satisfy Properties (1) and (2) if

$$
\rho^{-1}(|E(0)|)>1
$$

Moreover, since

$$
\rho\left(\left|E_{I}(j \omega)\right|\right) \leq \rho(|E(j \omega)|), \quad \forall I \varepsilon \quad \mathcal{J}, \omega
$$

placing one or more controllers $\mathrm{C}_{i}(\mathrm{~s})$ in manual simply relaxes the constraint in (14). Therefore the decentralized control system automatically satisfies property (3). 
Note that (15) is the definition of Generalized Diagonal Dominance for the plant $\mathrm{G}(0)$. This condition is independent of tuning parameters and is sufficient to guarantee that the corresponding control structure is acceptable.

Conditions equivalent to (14) and (15) exist for block-diagonal control structures but have found few, if any practical applications. The $\mu$ IM (Grosdidier and Morari, 1986). The theoretical foundations of the $\mu$ IM are identical to those of the DNA IM. However, the $\mu$ IM is less conservative and is in fact the tightest norm bound which can be placed on $H_{i}(j \omega)$, $(i=1, m)$.

Theorem 8. Assume that $\mathrm{G}(\mathrm{s})$ and $\tilde{\mathrm{H}}(\mathrm{s})$ are stable. Then the closed 1oop system $\mathrm{H}(\mathrm{s})$ is stable if

$$
\bar{\sigma}\left(H_{i}(j \omega)\right)<\mu^{-1}(E(j \omega)) \quad \forall i, \omega
$$

where $\bar{\sigma}(\mathbf{A})$ denotes the maximum singular value of the matrix $\mathbf{A}$ and $\mu(\mathbf{A})$ is the Structured Singular Value (SSV) of A (Doyle, 1982). In (17) $\mu(E(j \omega))$ is calculated with respect to the structure defined by $\tilde{G}(s)$. Note that (17) treats diagonal and block-diagonal control structures in a simple, unified manner.

A decentralized control system designed on basis of Theorem 8 can always satisfy properties (1), (2) and (3) if

$$
\mu^{-1}(\mathrm{E}(0))>1 \text {. }
$$

This condition is therefore sufficient to guarantee that the corresponding control structure is acceptable.

Rijnsdorp's IM (Rijnsdorp, 1965). Rijnsdorp's IM was introduced as a tool for the analysis of interactions in systems with 2 inputs and 2 outputs. Like the DNA and $\mu$ IM's, it can be used for pairing variables and tuning controllers. 
For any $2 \times 2$ plant $G(s)=\left\{g_{i j}(s)\right\}(i, j=1,2)$, Rijnsdorp's IM is defined as

$$
\kappa(s)=\frac{g_{12}(s) g_{21}(s)}{g_{11}(s) g_{22}(s)}
$$

the following equality shows the close relationship with the $\mu$ IM

$$
\mu(E(j \omega))=\sqrt{|\kappa(j \omega)|}
$$

and therefore Theorem 8 can be reformulated in terms of $\kappa(s)$ in the case of $2 \times 2$ systems. However, (17) is only a sufficient condition for the stability of $\mathrm{H}(\mathrm{s})$. This next theorem provides a necessary and sufficient condition.

Theorem 9. (Grosdidier and Morari, 1986). Assume that $\mathbf{G}(\mathrm{s})$ and $\tilde{\mathrm{H}}(\mathrm{s})$ are stable. Then the closed loop system $H(s)$ is stable if and only if

$$
N(1, z(s))=0
$$

where

$$
z(s)=\kappa(s) h_{1}(s) h_{2}(s)
$$

and where $\mathrm{N}(\mathrm{k}, \mathrm{g}(\mathrm{s}))$ denotes the net number of clockwise encirclements of the point $(k, 0)$ by the image of the Nyquist D contour under $g(s)$.

Trivially, a decentralized control system designed on the basis of Theorem 9 satisfies Properties (1)-(3).

Nyquist Stability Criterion. The multivariable Nyquist Stability Criterion provides a necessary and sufficient condition for the stability of a closed loop system $H(s)=G(s) C(s)(I+G(s) C(s))^{-1}$. Under the assumption that $\mathrm{G}(\mathrm{s})$ is open loop stable, $\mathrm{H}(\mathrm{s})$ will be stable if and only if

$$
\mathrm{N}(0, \operatorname{det}(\mathrm{I}+\mathrm{G}(\mathrm{s}) \mathrm{C}(\mathrm{s}))=0
$$

The Nyquist Stability Criterion can be used to address properties (1) and (3) of decentralized control systems. 
Performance Analysis. Although not explicitly included in our list of properties for decentralized control systems, good performance is an essential feature of any control system, irrespective of controller structure.

The performance of a control system can be analyzed via its sensitivity operator $(I+G(S) C(s))^{-1}$. This tool is needed to compensate for the fact that none of the IM's presented in this section provide controller tuning guidelines that will guarantee some performance specification for $\mathrm{H}(\mathrm{s})$.

Physically, the sensitivity operator is the transfer function matrix between disturbances acting at plant output and the tracking error. For good performance, its magnitude, i.e., $\bar{\sigma}\left((I+G(j \omega) C(j \omega))^{-1}\right)$ should be "small" at all frequencies. In practice, $\tilde{\sigma}\left((I+G(j \omega) C(j \omega))^{-1}\right)$ can be made small only at low frequencies. At higher frequencies, it will peak and subsequently take on an asymptotically constant value greater or equal to unity if $\mathrm{G}(\mathrm{s}) \mathrm{C}(\mathrm{s})$ is strictly proper. The peak indicates the frequency at which disturbances are amplified the most and its magnitude serves as an accepted measure of closed-10op performance. For good performance, and under typical conditions, it should be no greater than 2. Also important is the frequency at which $\overline{0}\left((I+G(j \omega) C(j \omega))^{-1}\right)$ first crosses the unit magnitude axis. This frequency, approximately equal to the bandwidth, also serves as a measure of closed-loop performance: all disturbances with lower frequencies will be immediately rejected by the closed-1oop system.

Discussion. The power of the RGA, BRG'S and NI is that they are computationally simple and provide sufficient conditions for rejecting 
unacceptable control structures. These features make the measures ideally suited for screening many alternative structures in a quick and efficient manner. Relative Gains and BRG's are unrelated to NI. Examples of control structures with positive RG's but negative NI can be constructed. Both measures are therefore useful for control structure selection.

Other steady state IM's, specifically Integral Controllability Eigenvalues, are of limited usefulness because they require an a priori knowledge of $\mathbf{K}_{\mathbf{i}}(0),(i=1, m)$. For this reason, these IM's will be given no further consideration in this communication.

The DNA and $\mu$ IM's are more powerful tools than the RGA, BRG's and NI since they can address both the control structure selection and the controller tuning problem. However, the $\mu$ IM is superior to the DNA IM because it is less conservative. Conservatism is the major stumbling block for any practical application of the DNA IM. Very few real systems are diagonally dominant and yet fewer are block-diagonally dominant. The use of a steady state decoupler to ensure diagonal dominance can serve as a palliative but will only defeat the purpose of decentralized control.

A drawback of both IM's is that they provide tuning guidelines that guarantee the stability of the closed loop system $H(s)$, but not its performance. The latter, in fact, can be arbitrarily poor. This point will later be illustrated in an example.

In the case of $2 \times 2$ systems, the chief benefit of Rijnsdorp's IM over the $\mu$ IM is that it uses both the gain and phase information contained in the elements of $\mathrm{G}(\mathrm{s})$. 
DECENTRALIZED CONTROL SYSTEM DESIGN METHODOLOGY

The design of a decentralized controller for a plant $G(s)$ is naturally decomposed into two successive operations: the selection of an acceptable control structure and the tuning of the controllers $\mathbf{c}_{i}(\mathrm{~s})$, $(i=1, m)$ such that properties (1)-(3) are satisfied. These operations will form the basis of our control system design methodology.

Control Structure Selection. Whereas a $2 \times 2$ plant offers two alternatives for decentralized control, a 3x3 plant offers 16 and a $4 \times 4$ plant 130. Evidently, the number of alternative structures grows rapidly with the number of plant inputs and outputs. For a given plant, the designer's task is to determine which of the many possible structures are acceptable.

It is only logical to start this search with the simplest structures and the simplest tools. For an $n \times n$ plant, there are $n !$ different alternatives for a fully-decentralized controller, each corresponding to a different set of variable pairings. The RGA and the NI of the plant can be used to sort out a large number of these: AlI structures associated with negative RG's or negative NI's can be eliminated from further considerations since they are known to be unacceptable. Calculation of $\mu(E(0))$ is only warranted for those control structures associated with positive RG's and NI. In turn, the calculation of $\mu(E(j \omega))$ over some specified frequency range is only justified if $\mu^{-1}(\mathbf{E}(0))>1$.

If a search fails to detect an acceptable diagonal control structure, more complex structures must be investigated. For an nxn plant, the simplest block diagonal control structure consists of a $2 \times 2$ controller block in parallel with $(n-2) 1 \times 1$ or SISO controllers. 
Undesirable control structures can again be eliminated by inspecting the signs of RG's, BRG's and NI's. The search for $2 \times 2$ blocks with positive BRG need not and should not be exhaustive; SISO blocks with positive RG's are easily identified and should be sought out first. There is simply no point in searching for $2 \times 2$ blocks with positive BRG unless the $(n-2) 1 \times 1$ blocks in the control structure themselves have positive RG's. Additional control structures can be eliminated by considering the RG's, $B R G^{\prime} s$ and NI's of submatrices $G_{I}(s), \forall I \varepsilon \mathcal{J}$. For the sake of clarity, this option will not be considered here.

This method of finding acceptable control structures generalizes in a trivial way to control structures of arbitrary complexity. Exhaustive searches will, in general, yield several acceptable control structures.

In the case of $2 \times 2$ systems, Rijnsdorp's IM may be more appropriate for selecting the variable pairings (Grosdidier and Morari, 1986). Controller Tuning. The constraints imposed by the $\mu$ IM or by Rijnsdorp's IM, (cf. (17) and (21)), serve as guidelines for tuning the controller blocks $C_{i}(s),(i=1, m)$. When single control loops $H_{i}(s)$ are stable, those guidelines will guarantee the stability of the complete system $H(s)$.

The performance of $\mathrm{H}(\mathrm{s})$ can be analyzed with the sensitivity operator $(\mathbf{I}+\mathbf{G}(\mathrm{s}) \mathbf{C}(\mathrm{s}))^{-1}$. Improved performance can be obtained by varying the bandwidth of the $H_{i}(s)(i=1, m)$ within the constraint set by $\mu^{-1}(E(j \omega))$. However, any such "fine tuning" effort is entirely trial and error. Implementation - Program DECENT. A salient feature of the mathematical tools presented in this communication is that most provide results in the form of Bode or Nyquist diagrams. This fact, coupled with the obvious need for computation implies that these tools are well- 
suited for computer implementation.

One such attempt at implementation was recently made at Caltech with the development of program DECENT. This program runs interactively and is a reflection of our methodology: Given an arbitrary plant $\mathbf{G}(\mathrm{s})$, a user can first address the control structure selection and subsequently the tuning of the controllers. Since our methodology can involve a large number of trials with alternate control structures and different controllers, simple scanning and updating features were emphasized.

Obviously, any such Interaction Analysis program will require a complimentary package for graphic display and for such tasks as the handling of frequency domain files and time domain simulation. In our case, much of this framework was preexistent in our Control System Design (CONSYD) package. As a result, DECENT conforms itself to the guidelines set by CONSYD and runs only on a VAX VMS operating system. Complimentary routines for matrix inversion and singular value decomposition were supplied by EISPACK and LINPACK, respectively. In this next section, DECENT will be used in the step-by-step design of a decentralized controller for a distillation system with four inputs and four outputs. 


\section{EXAMPLES}

Example 1. In this first example, DECENT will be used to investigate the feasibility of decentralized control for the $4 \times 4$ plant shown in Table 1 (Luyben, 1985). Controlled, manipulated and disturbance variables are listed in Table 2. We shall first investigate the feasibility of a diagonal controller and subsequently that of blockdiagonal controllers.

There are 24 possible diagonal control structures for the system in Table 1, each corresponding to a different set of variable pairings. Figure 3 shows the matrix of steady-state gains for the plant as well as its RGA (the data in this figure is presented in the same manner as the output of program DECENT).

Since a diagonal control structure must be based exclusively on variable pairings with positive relative gains, it is clear from Fig. 3 that the structure based on the (diagonal) pairings $\left(\left(\mathrm{u}_{1}, \mathrm{y}_{1}\right),\left(\mathrm{u}_{2}, \mathrm{y}_{2}\right)\right.$, $\left.\left(u_{3}, y_{3}\right),\left(u_{4}, y_{4}\right)\right)$ is the only one possibly acceptable. Any other set of pairings involves at least one negative relative gain which immediately rules out the possibility of decentralized control. Unfortunately, DECENT finds $\mu^{-1}(E(0))=0.61$ for a diagonal control structure based on diagonal pairings. Since this value is below unity, there is no guarantee that this control structure is acceptable.

Our next alternative is to investigate the feasibility of a blockdiagonal control structure. For a system with four inputs and four outputs, there are three kinds of block-diagonal control structures, each with a different number and different sizes of controller blocks. The simplest of these consists of a $2 \times 2$ controller block in series with 
two $1 \times 1$ controllers. There are 72 alternatives for such a structure, each corresponding to different variable pairings! There are 16 alternatives for a structure with a $3 \times 3$ and a $1 \times 1$ controller block and 18 alternatives for a structure with two $2 \times 2$ blocks (Table 3 ). In the first two cases, the RGA alone can be used to eliminate many unacceptable structures: A $1 \times 1$ controller should not be implemented on a pair of variables with a negative relative gain. Any such structure is certainly unacceptable.

Consider, for example, the control structures with a $3 \times 3$ and a $1 \times 1$ block. Figure 3 shows that for each variable pairing of the $1 \times 1$ controller, there corresponds only one $3 \times 3$ controller block. Since there are 16 possible pairings for the $1 \times 1$ controller, there are 16 possible control structures (Table 3). However, only 7 of these 16 pairings for the $1 \times 1$ controller are based on positive relative gains and are therefore possibly acceptable. With considerations to the RGA of $\mathrm{G}(\mathrm{s})$ only, more than half of the 16 alternatives have already been eliminated!

A similar analysis for structures with a $2 \times 2$ and two $1 \times 1$ controller blocks shows that only 16 of the 72 alternatives are possibly acceptable. We have so far managed to avoid calculating any BRG's. In the case of control structures with two $2 \times 2$ blocks, this is unavoidable unless one wishes to calculate $\mu^{-1}(E(0))$ for all 18 alternative structures.

Our search with program DECENT eventually led us to establish that there are 3 block diagonal control structures with $\mu^{-1}(E(0))>1$. Since this last condition is sufficient but not necessary for "acceptability", we conclude that there are at least 3 acceptable block-diagonal control 
structures for the system in Table 1. Table 4 summarizes these results and Fig. 4 shows plots of $\mu^{-1}(E(j \omega))$ for each of the 3 structures. The plots were obtained through program DECENT with CONSYD's plotting routines. Structure 1 has the most conservative $\mu^{-1}(\mathbf{E}(j \omega))$ plot but has the benefit of relative simplicity. For this reason, it will be the only one retained for further analysis among the three acceptable structures.

Example 2. Having selected a control structure for the plants in Table 1 (Structure 1 in Table 4), we shall now proceed and design the controller $\mathrm{C}(\mathrm{s})=\operatorname{diag}\left(\mathrm{C}_{1}(\mathrm{~s}), \mathrm{c}_{2}(\mathrm{~s}), \mathrm{c}_{3}(\mathrm{~s})\right)$. Here, $\mathrm{C}_{1}(\mathrm{~s})$ is a $2 \times 2$ controller which controls outputs $y_{1}$ and $y_{4}$ with inputs $u_{1}$ and $u_{4}$ and $c_{2}(s)$ and $c_{3}(s)$ are $1 \times 1$ controllers which control $y_{2}$ and $y_{3}$ with $u_{2}$ and $u_{3}$, respectively. Each of these controllers must be selected to make the closed-1oop transfer functions $H_{1}(s), h_{2}(s)$ and $h_{3}(s)$ stable and satisfy the magnitude bound set by Line 1 in Fig 4 .

For controller $\mathrm{C}_{1}(\mathrm{~s})$, we decided on a steady-state decoupler

$$
D=\left[\begin{array}{ll}
1.42 & 0.171 \\
3.55 & 1.42
\end{array}\right]
$$

precompensated by a diagonal matrix of PID controllers. For $c_{2}(s)$ and $c_{3}(s)$, we designed two PI controllers, each augmented with a first order lag. The controller parameters were selected by applying the IMC tuning rules (Rivera et al., 1986) to approximations of the transfer functions $g_{i i}(s),(i=1,4)$. Each of the controllers was tuned by gradually decreasing the "filter time constants" $\varepsilon_{i},(i=1,4)$ until $\sigma\left(H_{1}(j \omega)\right),\left|h_{2}(j \omega)\right|$ and $\left|h_{3}(j \omega)\right|$ approached the constraint set by $\mu^{-1}\left(E_{1}(j \omega)\right)$. Stability checks were also performed on $H_{1}(s), h_{2}(s)$ and $h_{3}(s)$ for each decrease in the time constants. Final tuning parameters are shown in Table 5 . 
Lines 1,2 and 3 in Fig. 5 are the Nyquist Loci of $\operatorname{det}\left(I+G_{1}(j \omega) C_{1}(j \omega)\right), 1+g_{22}(j \omega) C_{2}(j \omega)$ and $1+g_{33}(j \omega) c_{3}(j \omega)$, respectively. Since none of the loci encircles the origin of the complex plane, the stability of $\mathrm{H}_{1}(s), h_{2}(s)$ and $h_{3}(s)$ is guaranteed. Lines 1,2 and 3 in Fig. 6 are the plots of $\bar{\sigma}\left(H_{1}(j \omega)\right),\left|h_{2}(j \omega)\right|,\left|h_{3}(j \omega)\right|$, respectively. Since these lines remain constrained by the plot of $\mu^{-1}\left(E_{1}(j \omega)\right.$ ) (Line 4$)$, the stability of the complete system $\mathrm{H}(\mathrm{s})$ is guaranteed.

Finally, Fig. 7 shows a plot of $\bar{\sigma}\left((I+G(j \omega) C(j \omega))^{-1}\right)$. This figure shows that our performance measure peaks at a magnitude of 5 , a value above what is generally desired. Fine tuning of the controllers can be expected to reduce the magnitude of the peak but was not attempted here.

No control system design is complete without time-domain simulation of the closed loop system. In this particular case, simulation was performed by one of CONSYD's simulation programs. Using this program, we have simulated responses of the closed-loop system for setpoint and disturbance changes. Output responses to unit step changes in $r_{2}$ and $r_{3}$ are shown in Fig. 8 and 9, respectively. Responses to a unit step disturbance are shown in Fig. 10.

Figures 8 and 9 show that interactions affect different outputs to varying degrees. This is a result of the fact that interactions have direction in addition to magnitude. Only the latter is quantified by the IM's discussed in this communication.

An understanding of how the direction of interactions affect performance might yield guidelines for the selection of appropriate "filter time constants" for each controller $C_{j}(s),(i=1,3)$. 
Example 3. See Grosdidier and Morari (1985) for an illustration of Rijnsdorp's IM.

Example 4. The inability to find an acceptable control structure for a given plant may indicate more than the unsuitability of decentralized control. It may also indicate that the plant under consideration is inherently difficult to control. This point can be illustrated by considering a pair of $2 \times 2$ plants $G_{1}(s)$ and $G_{2}(s)$ with steady-state gain matrices

$$
G_{1}(0)=\left[\begin{array}{cc}
10 & 10 \\
-9 & 10
\end{array}\right] ; \quad G_{2}(0)=\left[\begin{array}{cc}
10 & 10 \\
9 & 10
\end{array}\right]
$$

A control structure based on diagonal pairings yields

$$
\mu^{-1}\left(E_{1}(0)\right)=\mu^{-1}\left(E_{2}(0)\right)=1.05
$$

A value of $\mu^{-1}(E(0))$ so close to unity indicates strong interactions in both plants and therefore decentralized control - although possible, is not recommended.

However, interactions within these two plants are not the same. This will become evident after inspection of the RGAs for $\mathbf{G}_{1}(0)$ and $\mathbf{G}_{2}(0)$ :

$$
\Lambda_{1}=\left[\begin{array}{ll}
0.53 & 0.47 \\
0.47 & 0.53
\end{array}\right] ; \Lambda_{2}=\left[\begin{array}{cc}
10 & -9 \\
-9 & 10
\end{array}\right]
$$

Whereas $\Lambda_{1}$ has relative gains between zero and unity, $\Lambda_{2}$ has relative gains with magnitude much larger than one. It is now well-established that large relative gains are the trademark of "ill-conditioned" plants (Grosdidier et al., 1985). These plants display high sensitivity to modeling error and, as a result, can be inherently difficulty to control. Altematively, plants with relative gains between zero and unity are well-behaved and, when strongly interactive as in the case of $G_{1}(s)$, can 
be easily controlled with a multivariable controller.

This example shows that interactions in plants can differ in ways undetected by the $\mu$ IM, yet with great implications with regards to the feasibility of control, whether decentralized or not. This fact should be borne in mind whenever an attempt is made to design a decentralized controller. It also stresses, once again, the value of the RGA as a complementary tool to the $\mu$ IM.

\section{CONCLUSION}

The use of decentralized controllers in the chemical process industries is justified if tuning is simplified and the resulting control system is failure tolerant. The ability to satisfy these criteria depends on the choices of control structure and tuning parameters. Interaction Measures (IM's) are analysis tools which assist the designer in addressing each of these choices in a rigorous, quantitative manner.

In terms of usefulness, two IM's stand out distinctively among others: The Relative Gain (RG) IM and the $\mu$ IM. The first, by virtue of its computational simplicity, is a powerful tool for screening many alternative control stmuctures in a quick and efficient manner. Undesirable control structures can be rejected after mere inspection of the sign of their RG's. Control structures which passed this test can be subsequently analyzed in greater detail with the $\mu$ IM. For appropriate control structures this measure provides simple tuning guidelines which guarantee, among other things, the stability and failure tolerance of the closed loop system. Unfortunately, the guidelines provide no guarantee of closed loop performance. 
Along with other control system analysis tools, these IM's form the basis of a simple methodology for the design of decentralized controllers. A program developed at Caltech within the framework of the CONSYD package reflects this methodology. 
APPENDIX

The theorems will be proved for $\Lambda_{11}$ but are valid for any $\Lambda_{i j}$, $(i=1, m)$.

Proof of Theorem 3. Let $I_{1}=[2,3, \ldots, \mathrm{m}]$ and express $\mathbf{G}(0)$ as

$$
G(0)=\left[\begin{array}{ll}
G_{11}(0) & G_{1 \Gamma}(0) \\
G_{1 C}(0) & G_{I_{1}}(0)
\end{array}\right]
$$

then

$$
\Lambda_{11}=G_{11}(0) \cdot \hat{G}_{11}(0)
$$

can be expressed as

$$
\Lambda_{11}=\left(I-G_{1 \Gamma}(0) G_{I_{1}}^{-1}(0) G_{1 C}(0) G_{11}^{-1}(0)\right)^{-1}
$$

Applying Schur's formula (Gantmacher, 1959) yields

$$
\operatorname{det}\left(\Lambda_{11}\right)=\frac{\operatorname{det}\left(\mathbf{G}_{11}(0)\right) \operatorname{det}\left(\mathbf{G}_{I_{1}}(0)\right)}{\operatorname{det}(\mathbf{G}(0))}
$$

Although $\Lambda_{11}$ is scaling dependent, $\operatorname{det}\left(\Lambda_{11}\right)$ is scaling invariant for block-diagonal scaling corresponding to the partitioning in (A1). Therefore

$$
\operatorname{det}\left(\Lambda_{11}\right)=\frac{\operatorname{det}\left(\mathbf{G}_{11}(0) \mathbf{K}_{1}(0)\right) \cdot \operatorname{det}\left(\mathbf{G}_{\mathrm{I}_{1}}(0) \mathbf{K}_{\mathrm{I}_{1}}(0)\right)}{\operatorname{det}(\mathbf{G}(0) \mathbf{K}(0))}
$$

If $\operatorname{det}\left(\Lambda_{11}\right)<0$, then one or three of the terms in (A5) is negative. Using theorem 1 of Grosdidier et al. (1985), we get, for property (a) $\operatorname{det}(\mathrm{G}(0) \mathrm{K}(0))<0$; for property (b) $\operatorname{det}\left(\mathrm{G}_{11}(0) \mathrm{K}_{1}(0)\right)<0$; for property (c) $\operatorname{det}\left(\mathrm{G}_{\mathrm{I}_{1}}(0) \mathrm{K}_{\mathrm{I}_{1}}(0)\right)<0$. 
Proof of Theorem 6. When $\mathrm{G}_{\mathrm{I}_{1}}$ (s) is under feedback, the transfer function matrix between input vector $u_{1}$ and output vector $y_{1}$ is effectively

$$
\left.G_{11}, C L(s)=G_{11}(s)-G_{1 \Gamma}(s)\left(I+G_{I_{1}}(s) C_{I_{1}}(s)\right)^{-1} C_{I_{1}}(s)\right) G_{1 C}(s)
$$

If $s=0$

$$
G_{11, C L}(0)=G_{11}(0)-G_{1 \Gamma}(0) G_{I_{1}}^{-1}(0) G_{1 C}(0)
$$

Using (A3),

$$
G_{11, C L}(0)=\Lambda_{11}^{-1} G_{11}(0)
$$

QED 
REFERENCES

Alatiqi, I. M. and W. L. Luyben (1985). Control of a Complex Sidestream Column/Stripper Distillation Configuration. Accepted for publication, Ind. Eng. Chem. Process Des. Dev.

Bristol, E. H. (1966). On a New Measure of Interaction for Multivariable

Process Control. IEEE Trans. Autom. Control, AC-11, 133-134.

Doyle, J. (1982). Analysis of Feedback Systems with Structured

Uncertainties. IEE Proc., 129, 242-250.

Economou, C. and M. Morari (1986). Internal Model Control. 6.

Multiloop Design. In press, Ind. Eng. Chem. Process Des. Dev.

Gantmacher, F. R. (1959). Matrix Theory. Chelsea Publishing Co.,

New York.

Grosdidier, P., M. Morari and B. R. Holt (1985). Closed-Loop

Properties from Steady-State Gain Information. Ind. Eng. Chem.

Fund., 24, 221-235.

Grosdidier, P. and M. Morari (1985). The $\mu$ Interaction Measure.

Submitted to Ind. Eng. Chem. Fund.

Grosdidier, P. and M. Morari (1986). Interaction Measures for Systems

Under Decentralized Control. In press, Automatica.

Limebeer, D. J. N. (1982). The Application of Generalized Diagonal

Dominance to Linear System Stability Theory. Int. J. Control,

$36,185-212$.

Manousiouthakis, V. and Y. Arkun (1985). Selection of Decentralized

Process Control Structures, Proceedings of the American Control

Conference, Boston, 299-304.

Mees, A. I. (1981). Achieving Diagonal Dominance. Systems and Control 
Letters, $1,155-158$.

Morari, M. (1985). Robust Stability of Systems with Integral Control. IEEE Trans. Autom. Control, AC-30, 574-577.

Rijnsdorp, J. E. (1965). Interaction in Two-Variable Control Systems for Distillation Columns - I. Automatica, 1, 15-28.

Rivera, D. E., M. Morari and S. Skogestad (1986). Internal Model Control. 4. PID Controller Design. Ind. Eng. Chem. Process Des. Dev., 25, 252-265.

Rosenbrock, H. H. (1974). Computer-Aided Control System Design. Academic Press, London.

Skogestad, S. (1985). Personal communication. 
Table 1. Distillation Column Transfer Function Matrix

\begin{tabular}{|c|c|c|c|c|c|}
\hline & $u_{x}$ & $\mathrm{u}_{2}$ & $u_{3}$ & $u_{4}$ & $d$ \\
\hline $\mathrm{y}_{1}$ & $\frac{4.09 e^{-1.3 s}}{(33 s+1)(8.3 s+1)}$ & $\frac{-6.36 e^{-0.2 s}}{(31.6 s+1)(20 s+1)}$ & $\frac{-0.25 e^{-0.4 s}}{(21 s+1)}$ & $\frac{-0.49 e^{-5 s}}{(22 s+1)^{2}}$ & $\frac{-0.86 e^{-6 s}}{(19.2 s+1)^{2}}$ \\
\hline $\mathrm{y}_{2}$ & $\frac{-4.17 e^{-4 s}}{(45 s+1)}$ & $\frac{6.93 e^{-1.01 s}}{(44.6 s+1)}$ & $\frac{-0.05 e^{-5 s}}{(34.5 s+1)^{2}}$ & $\frac{1.53 e^{-2.8 s}}{(48 s+1)}$ & $\frac{-1.06 e^{-5 s}}{(35 s+1)}$ \\
\hline $\mathrm{y}_{3}$ & $\frac{-1.73 e^{-17 s}}{(13 s+1)^{2}}$ & $\frac{5.11 e^{-11 s}}{(13 \cdot 3 s+1)^{2}}$ & $\frac{4.61 e^{-1.02 s}}{(18.5 s+1)}$ & $\frac{-5.48 e^{-0.5 s}}{(15 s+1)}$ & $\frac{1.2 e^{-9 s}}{(24 s+1)}$ \\
\hline $\mathrm{y}_{4}$ & $\frac{-11.18 e^{-2.6 s}}{(43 s+1)(6.5 s+1)}$ & $\frac{14.04 e^{-0.02 s}}{(45 s+1)(10 s+1)}$ & $\frac{-0.1 e^{-0.05 s}}{(31.6 s+1)(5 s+1)}$ & $\frac{4.49 e^{-0.6 s}}{(48 s+1)(6.3 s+1)}$ & $\frac{-0.86 e^{-0.2 s}}{\left(16 s^{2}+4 s+1\right)}$ \\
\hline
\end{tabular}


Table 2. Controlled, Manipulated and Disturbance Variables

$\mathrm{y}_{1}=$ Benzene purity in distillate of first column

$\mathrm{y}_{2}=$ Xylene purity in bottoms of first column

$\mathrm{y}_{3}=$ Toluene purity in bottoms of second column

$\mathrm{y}_{4}=$ Temperature difference across the first column

$u_{1}=$ Reflux ratio of first column

$u_{2}=$ Reboil duty of first column

$u_{3}=$ Reboil duty of second column

$u_{4}=$ Liquid draw from first to second column

$\mathrm{d}=$ Toluene concentration in feed of first column 
Table 3. Alternative Control Structures for the System in Table 1

\begin{tabular}{llll}
\hline $\begin{array}{l}\text { Control Structure } \\
\text { Form }\end{array}$ & $\begin{array}{l}\text { Number of } \\
\text { Alternative } \\
\text { Structures }\end{array}$ & $\begin{array}{l}\text { Number of Structures } \\
\text { with positive RG' }\end{array}$ & $\begin{array}{l}\text { Number of } \\
\text { Structures } \\
\text { with } \\
\mu^{-1}(\mathrm{E}(0))>1\end{array}$ \\
\hline $4(1 \times 1)$ blocks & 24 & 1 & 0 \\
\hline $2(1 \times 1)$ blocks & 72 & 15 & 1 \\
$1(2 \times 2)$ block & & 7 & 2 \\
\hline $1(1 \times 1)$ block & 16 & - & 0 \\
$1(3 \times 3)$ block & 18 & & \\
\hline $2(2 \times 2)$ blocks & & & \\
\hline
\end{tabular}

* Note that this column does not consider BRG's. 
Table 4. Acceptable Control Structures for the System in Table 1

\begin{tabular}{|c|c|c|c|c|}
\hline Structure & $\begin{array}{l}\text { Number } \\
\text { of Blocks }\end{array}$ & $\begin{array}{l}\text { Block } \\
\text { Dimensions }\end{array}$ & $\begin{array}{l}\text { Variable } \\
\text { Pairings }\end{array}$ & $\mu^{-1}(E(0))$ \\
\hline 1 & 3 & $\begin{array}{l}2 \times 2 \\
1 \times 1 \\
1 \times 1\end{array}$ & $\begin{array}{l}\left(u_{1}, y_{1}\right) \\
\left(u_{4}, y_{4}\right) \\
\left(u_{2}, y_{2}\right) \\
\left(u_{3}, y_{3}\right)\end{array}$ & 1.06 \\
\hline 2 & 2 & $\begin{array}{l}3 \times 3 \\
1 \times 1\end{array}$ & $\begin{array}{l}\left(u_{1}, y_{1}\right) \\
\left(u_{3}, y_{3}\right) \\
\left(u_{4}, y_{4}\right) \\
\left(u_{2}, y_{2}\right)\end{array}$ & 1.08 \\
\hline 3 & 2 & $\begin{array}{l}3 \times 3 \\
1 \times 1\end{array}$ & $\begin{array}{l}\left(u_{1}, y_{1}\right) \\
\left(u_{2}, y_{2}\right) \\
\left(u_{4}, y_{4}\right) \\
\left(u_{3}, y_{3}\right)\end{array}$ & 1.65 \\
\hline
\end{tabular}


Table 5. PID Controller Tuning Parameters

\begin{tabular}{lllll}
\hline & $C_{1}(s)^{1}$ & $c_{2}(s)$ & $c_{3}(s)$ \\
\hline$k_{i}$ & 0.474 & 0.587 & 0.292 & 0.182 \\
$1 / \tau_{i}, \min$ & 0.0242 & 0.0184 & 0.0224 & 0.054 \\
$\tau_{D}, \min$ & 6.63 & 5.57 & 0. & 0. \\
$\tau_{F}{ }^{2}, \min$ & 0.663 & 0.557 & 0.917 & 0.926 \\
\hline
\end{tabular}

Notes:

(1) These are the parameters for the two PID controllers in $C_{1}(s)$. The first order lag time constants were chosen as $1 / 10$ of $\tau_{D}$ and are there simply to ensure the properness of the controller.

(2) $\tau_{F}$ is the first order lag time constant. 


\section{FIGURE CAPTIONS}

Figure 1. General decentralized control structure.

Figure 2. Decentralized integral control configuration.

Figure 3. Steady state gain matrix and RGA for the system in Table 1.

Figure 4. $\mu$ IMs. Line $1=\mu^{-1}\left(E_{1}(j \omega)\right)$; Line $2=\mu^{-1}\left(E_{2}(j \omega)\right)$; Line $3=$ $\mu^{-1}\left(E_{3}(j \omega)\right)$.

Figure 5. Nyquist Loci. Line $1=\operatorname{det}\left(I+G_{11}(j \omega) C_{1}(j \omega)\right)$; Line $2=$ $1+g_{22}(j \omega) c_{2}(j \omega)$; Line $3=1+g_{33}(j \omega) c_{3}(j \omega)$.

Figure 6. Constraint check. Line $1=\bar{\sigma}\left(\mathrm{H}_{1}(j \omega)\right)$; Line $2=\left|\mathrm{h}_{2}(j \omega)\right|$; Line $3=\left|h_{3}(j \omega)\right| ;$ Line $4=\mu^{-1}\left(E_{1}(j \omega)\right)$.

Figure 7. Sensitivity operator.

Figure 8. Closed loop responses for unit step change in $r_{2}(s)$.

Figure 9. Closed loop responses for unit step change in $r_{3}(s)$.

Figure 10. Closed loop responses for unit step change in $d(s)$. 


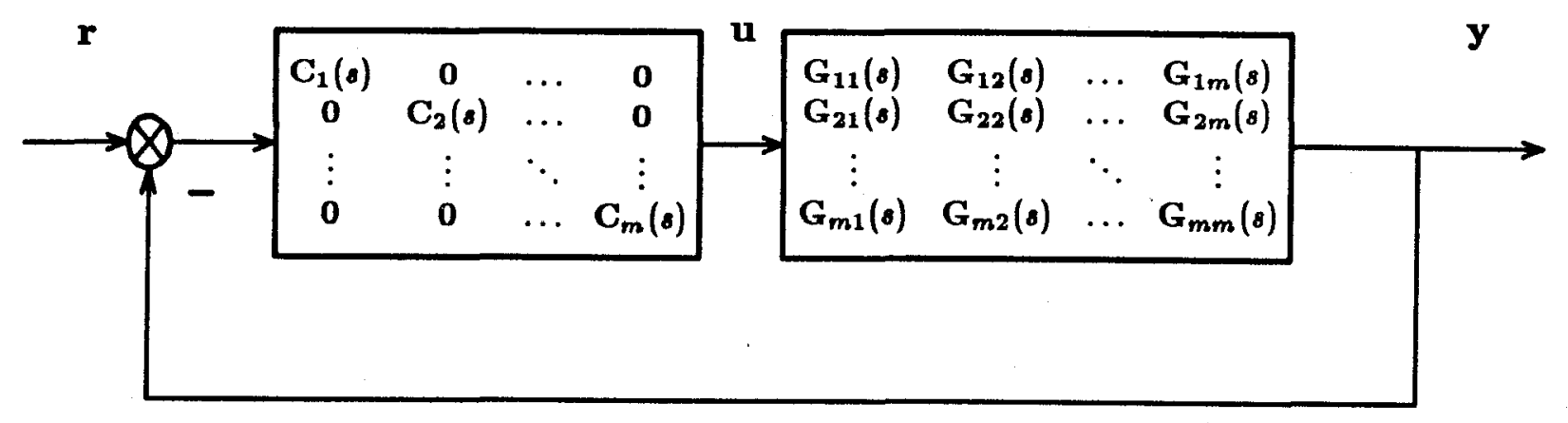

Figure 1. General decentralized control structure.

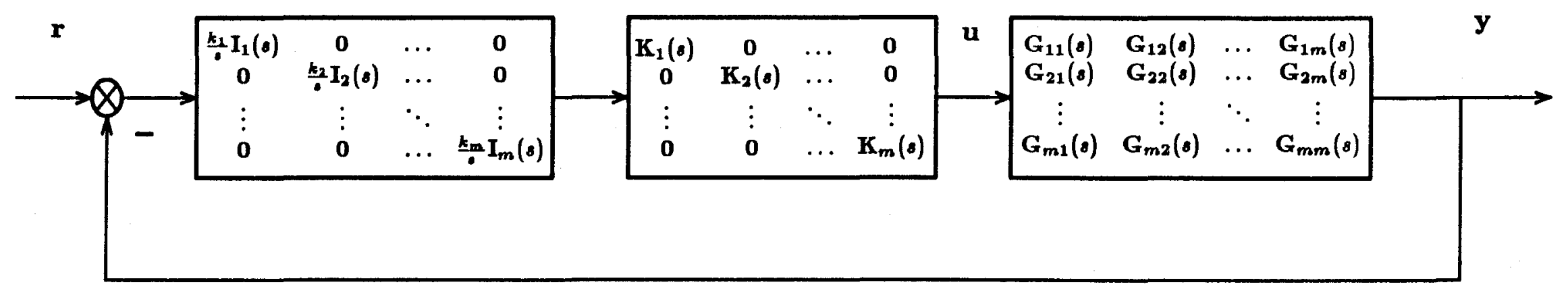

Figure 2. Decentralized integral control configuration. 


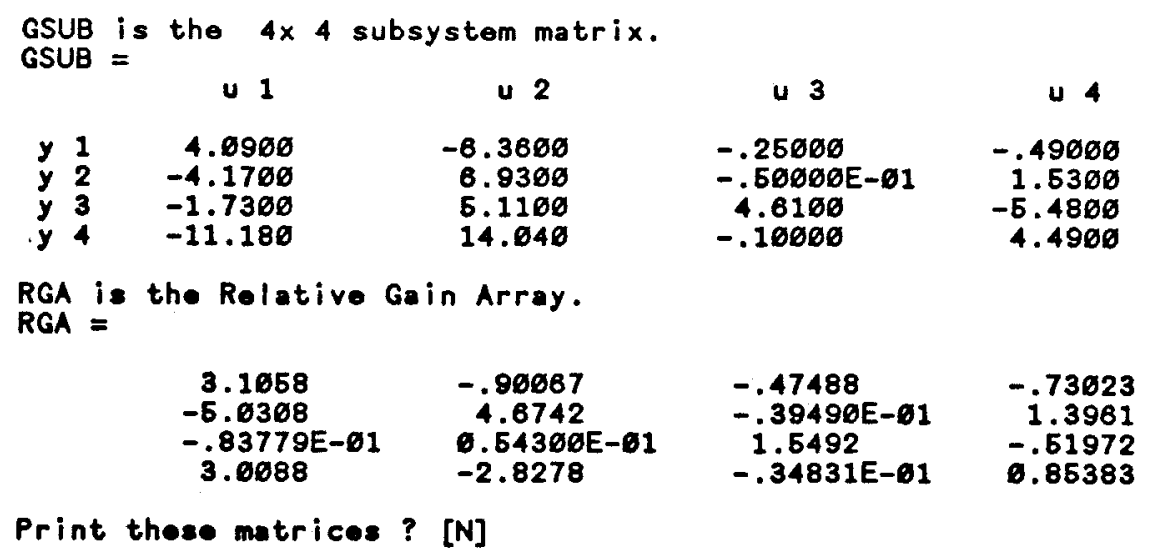

Figure 3. Steady state gain matrix and RGA for the system in Table 1.

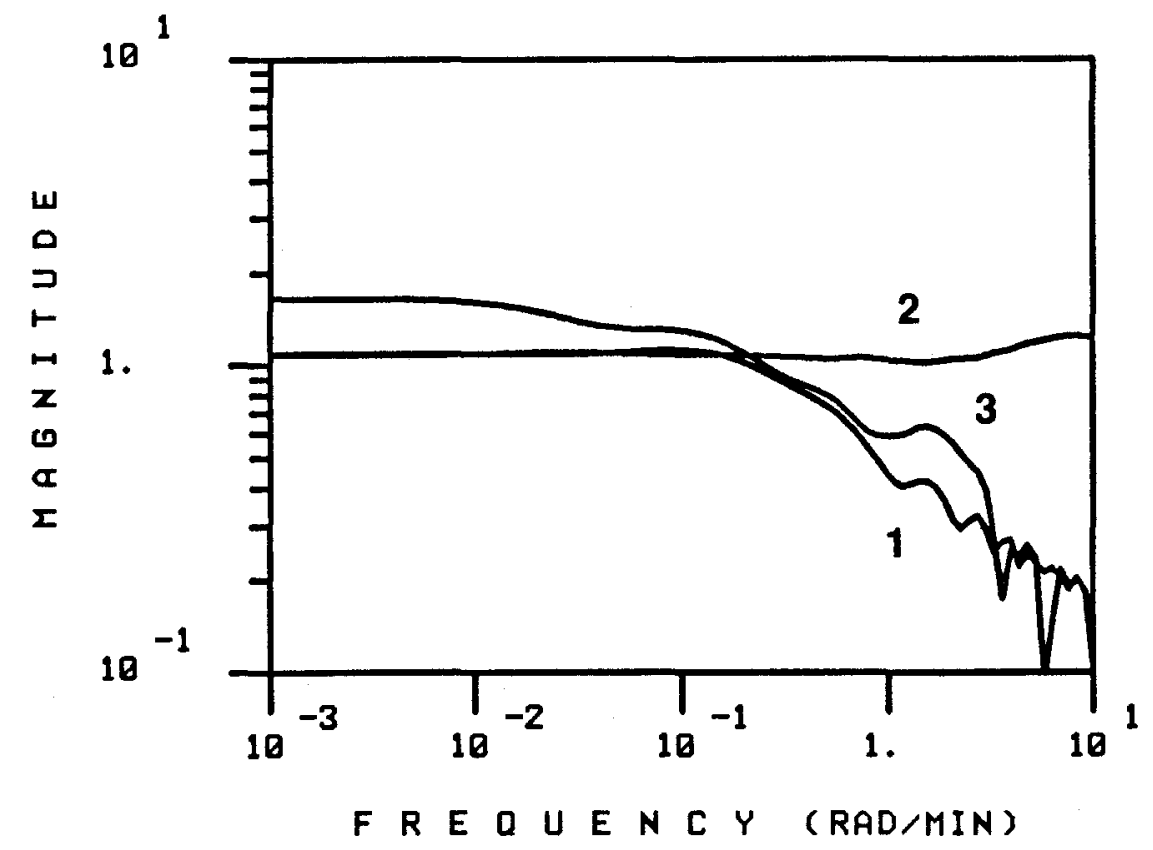

Figure 4. $\mu$ IMs. Line $1=\mu^{-1}\left(E_{1}(j \omega)\right)$; Line $2=\mu^{-1}\left(E_{2}(j \omega)\right)$; Line $3=$ $\mu^{-1}\left(E_{3}(j \omega)\right)$. 


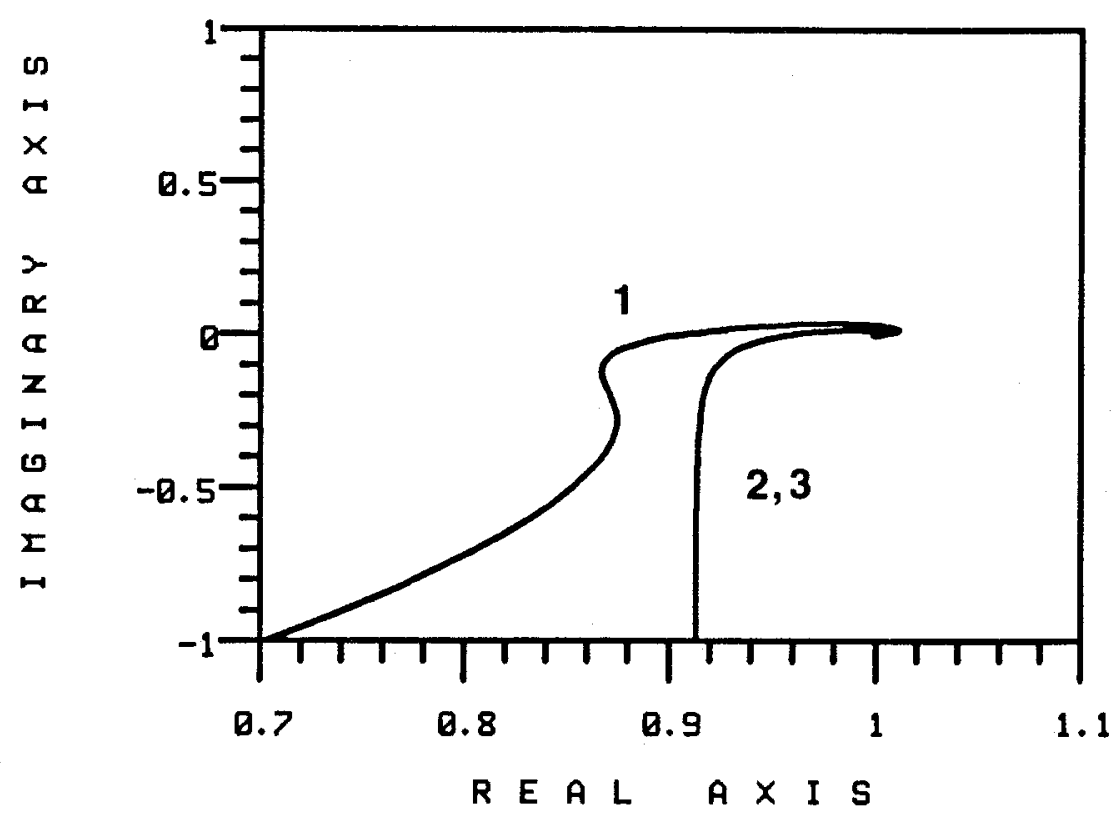

Figure 5. Nyquist Loci. Line $1=\operatorname{det}\left(I+G_{11}(j \omega) C_{1}(j \omega)\right)$; Line $2=$ $1+g_{22}(j \omega) c_{2}(j \omega) ;$ Line $3=1+g_{33}(j \omega) c_{3}(j \omega)$.

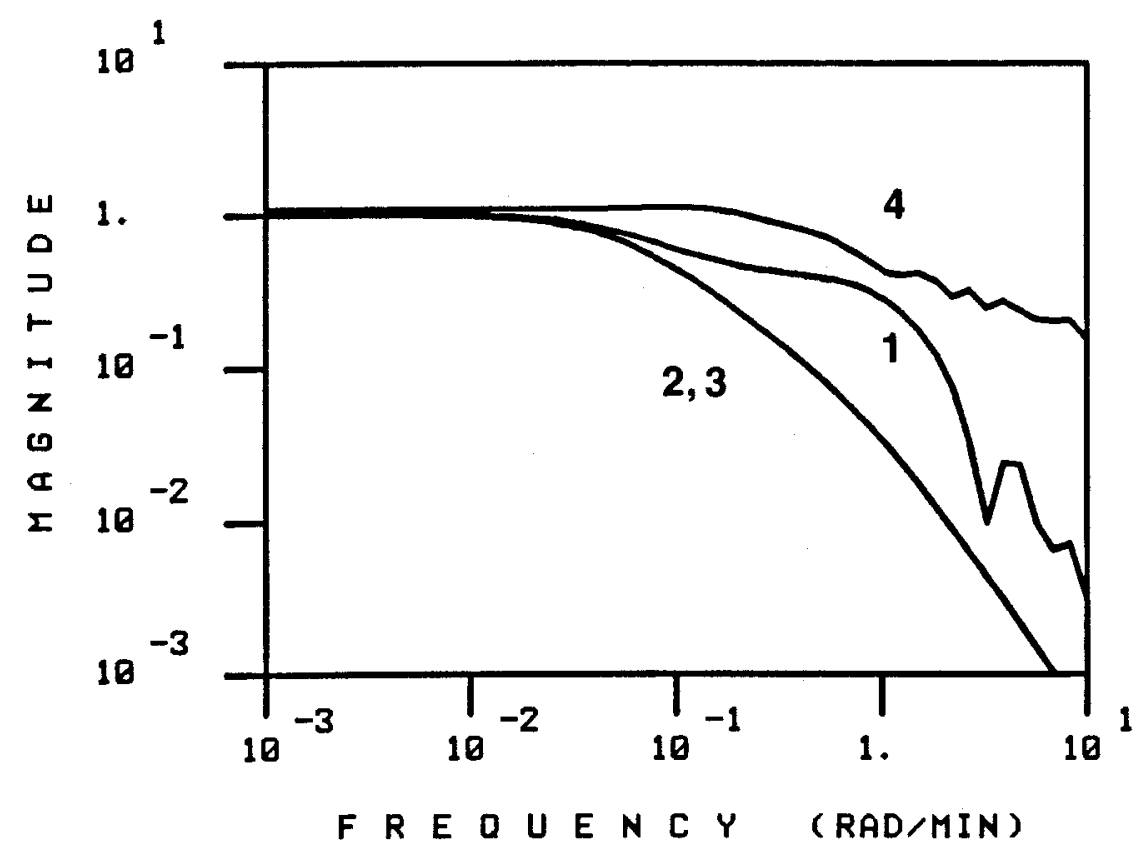

Figure 6. Constraint check. Line $1=\bar{\sigma}\left(\mathrm{H}_{2}(j \omega)\right)$; Line $2=\left|\mathrm{h}_{2}(j \omega)\right|$; Line $3=\left|h_{3}(j \omega)\right| ;$ Line $4=\mu^{-1}\left(E_{1}(j \omega)\right)$. 


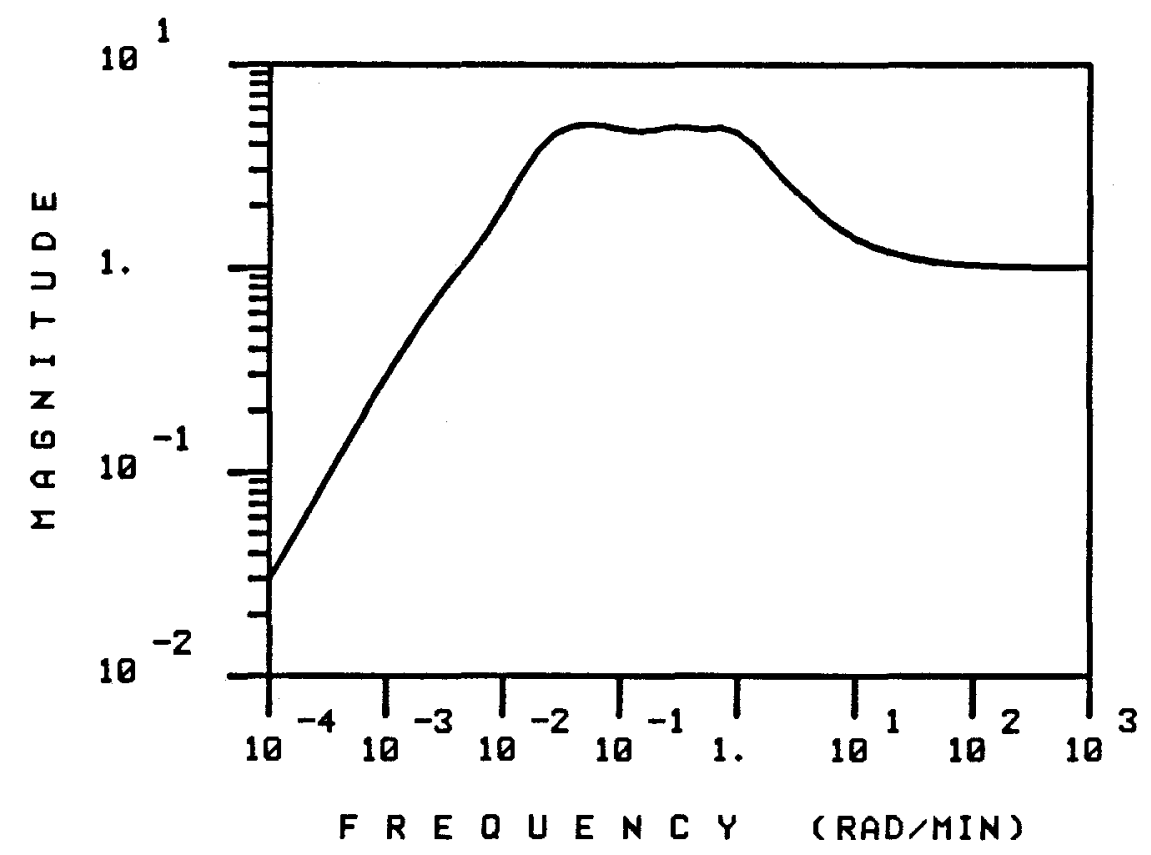

Figure 7. Sensitivity operator.

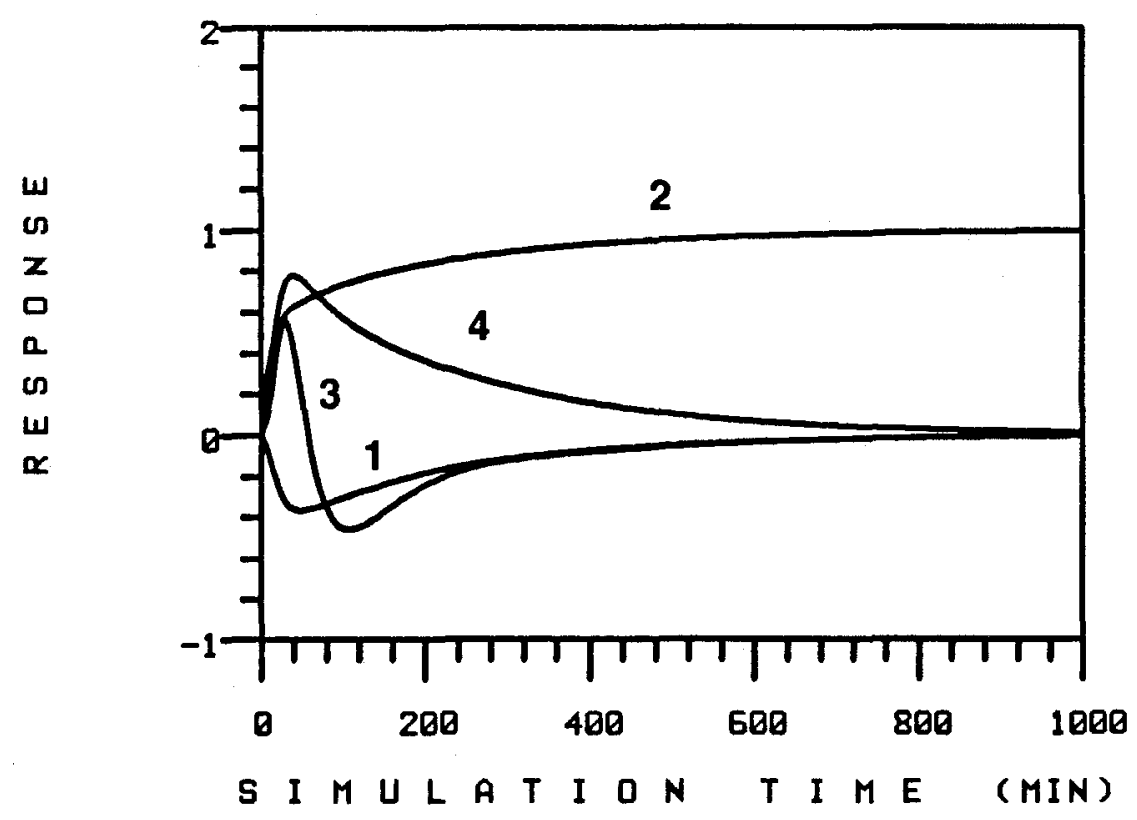

Figure 8. Closed loop responses for unit step change in $r_{2}(s)$. 


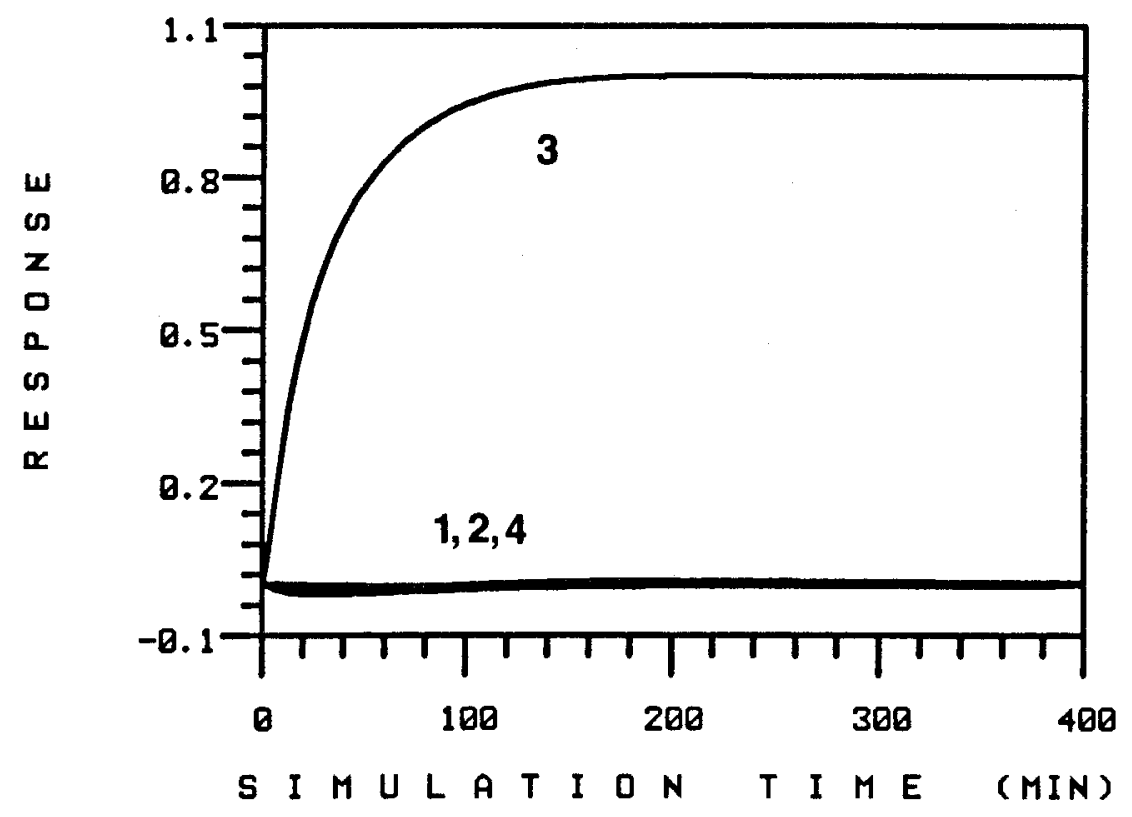

Figure 9. Closed loop responses for unit step change in $r_{3}(s)$.

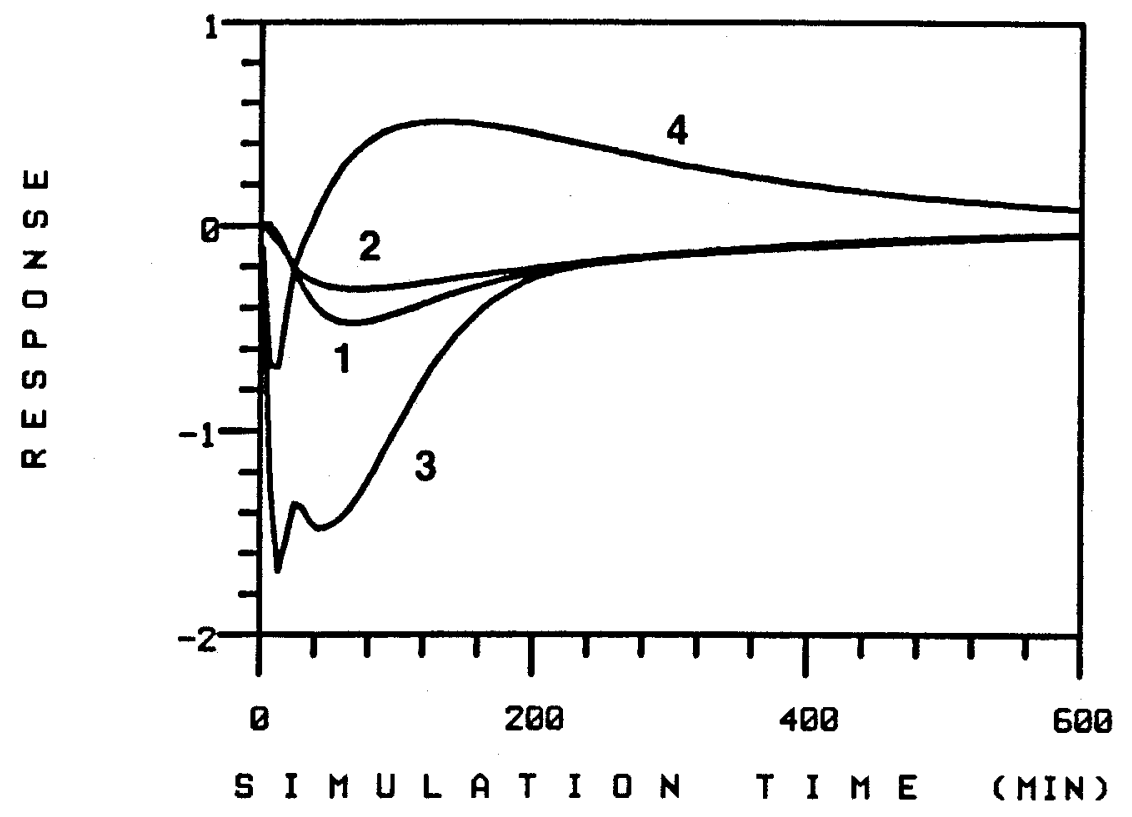

Figure 10. Closed loop responses for unit step change in $d(s)$. 
210

CHAPTER VI: CONCLUSIONS AND RECOMMENDATIONS 
This research project has motivated the use of decentralized controllers and proposed a new approach to their design. For our further developments, let $\mathbf{G}(s)=\left\{\mathbf{G}_{i j}(s)\right\},(i, j=1, m)$ denote a multivariable plant and let

$$
\mathbf{C}(s)=\operatorname{diag}\left(\mathbf{C}_{1}(s), \mathbf{C}_{2}(s), \ldots \mathbf{C}_{m}(s)\right)
$$

and

$$
\tilde{\mathbf{G}}(s)=\operatorname{diag}\left(\mathbf{G}_{11}(s), \mathbf{G}_{22}(s), \ldots \mathbf{G}_{m m}(s)\right)
$$

denote the diagonal or block-diagonal controller and the corresponding blockdiagonal plant, respectively.

The basis of our approach is to rely on Interaction Measures (IM's) to express the constraints imposed on the choice of $\tilde{\mathbf{G}}(s)$ and $\mathbf{C}(s)$ such that the stability of the block-diagonal system

$$
\tilde{\mathbf{H}}(s)=\tilde{\mathbf{G}}(s) \mathbf{C}(s)(\mathbf{I}+\tilde{\mathbf{G}}(s) \mathbf{C}(s))^{-1}
$$

guarantees that of the complete closed-loop system

$$
\mathbf{H}(s)=\mathbf{G}(s) \mathbf{C}(s)(\mathbf{I}+\mathbf{G}(s) \mathbf{C}(s))^{-1} .
$$

Using the notion of Structured Singular Values, we have defined a new dynamic IM for systems of arbitrary size under feedback with diagonal or blockdiagonal controllers. This measure, the $\mu \mathrm{IM}$, is a function of the "relative error matrix"

$$
\mathbf{E}(s)=(\mathbf{G}(s)-\tilde{\mathbf{G}}(s)) \tilde{\mathbf{G}}^{-1}(s)
$$

and the structure of the controller. By structure, we mean the number and size of the controller blocks. Assuming that $\mathbf{G}(s)$ and $\tilde{\mathbf{H}}(s)$ are stable, the closed-loop system $\mathbf{H}(s)$ will be stable if the inequality

$$
\bar{\sigma}\left(\mathbf{H}_{i}(j \omega)\right)<\mu^{-1}(\mathbf{E}(j \omega)) \quad \forall i, \omega
$$


is satisfied. In this last expression, $\bar{\sigma}(\mathbf{A})$ denotes the maximum singular value of the matrix $\mathbf{A}$ and $\mathbf{H}_{i}(s),(i=1, m)$ are the diagonal blocks of the matrix $\tilde{\mathbf{H}}$. Equation (6) states that for stability the magnitude of the diagonal blocks $\mathbf{H}_{i}(s)$ has to be constrained by the reciprocal of the $\mu \operatorname{IM}(\mu(\mathbf{E}(j \omega)))$. The blockdiagonal plant $\tilde{\mathbf{G}}(s)$ must therefore be selected to minimize this constraint. In the absence of synthesis tools, the block-diagonal plant $\tilde{\mathbf{G}}(s)$ that minimizes the constraint can only be found through an exhaustive search of all possible $\tilde{\mathbf{G}}(s)^{\prime} s$. For a plant with more than two inputs and two outputs, this represents a long and tedious task. Fortunately, we have shown how the Relative Gain Array (RGA) can be of considerable help in the selection process. The RGA provides a computationally simple condition based upon which a choice of $\tilde{\mathbf{G}}(s)$ can be rejected as inadequate.

The benefits of our approach to the design of decentralized controllers are as follows.

(1) Simple design. The RGA and the $\mu$ IM provides simple, quantitative guidelines for choosing $\tilde{\mathbf{G}}(s)$ and $\mathbf{C}(s)$.

(2) Decentralized tuning. The controllers $\mathbf{C}_{i}(s),(i=1, m)$ are tuned to make each of the closed-loop systems $\mathbf{H}_{i}(s)=\mathbf{G}_{i i}(s) \mathbf{C}_{i}(s)\left(\mathbf{I}+\mathbf{G}_{i i}(s) \mathbf{C}_{i}(s)\right)^{-1}$ stable.

(3) Failure tolerance. The decentralized control system remains stable when failure occurs in any number of feedback loops.

The drawbacks are as follows.

(1) Performance. The $\mu$ IM provides tuning guidelines that guarantee the stability of the closed loop system $\mathbf{H}(s)$ but not its performance. When designing a decentralized controller using our methodology, the temptation is great to tune the controllers $\mathbf{C}_{i}(s),(i=1, m)$ such that the closed-loop blocks 
$\mathbf{H}_{i}(s),(i=1, m)$ all roll-off at the same frequency imposed by the $\mu$ IM (cf. (6)). In general, our examples show that this approach does not yield a closed-loop system $\mathbf{H}(s)$ with good performance. Each controller $\mathbf{C}_{\boldsymbol{i}}(s)$ must be fine-tuned by trial and error within the constraints set by the $\mu$ IM in order to improve the quality of closed-loop performance.

(2) Direction of interactions. The $\mu \mathrm{IM}$ quantifies the magnitude of interactions in a plant under decentralized control but gives no measure of their direction. The latter has an important effect on performance as illustrated by the examples in this thesis. Controller tuning guidelines that guarantee that $\mathbf{H}(s)$ satisfies some performance objective will be derived only after the direction of interaction is quantified and its effect on performance is understood.

These two issues, understanding the effect of interaction direction and finding tuning guidelines for performance, are closely related and remain outstanding for future research.

Finally, the fact that the examples in this thesis display only marginally acceptable closed-loop performance is due, in parts, to the very strong interactions within the plants under consideration. Our approach showed that in spite of the strong interactions, these plants can be controlled with a decentralized controller. However, the price to pay in closed-loop performance suggests that a more complex multivariable controller might be more appropriate. Future research efforts should also focus on deriving bounds on the achievable performance of a closed-loop system with a decentralized controller. Such bounds will be useful in deciding whether or not decentralized control is desirable whenever the $\mu$ IM indicates that this policy is possible. 